\title{
STUDY OF NEUTRINO INTERACTIONS IN MINOS
}

\author{
A THESIS \\ submitted to the \\ FACULTY OF SCIENCE \\ PANJAB UNIVERSITY, CHANDIGARH \\ for the degree of \\ DOCTOR OF PHILOSOPHY
}

2014

RICHA SHARMA

DEPARTMENT OF PHYSICS

CENTRE OF ADVANCED STUDY IN PHYSICS

PANJAB UNIVERSITY, CHANDIGARH

INDIA 



\section{Acknowlegements}

The work presented in this thesis has been made possible with the help of the many MINOS collaborators and the support of my family and friends. First and foremost, I would like to thank my supervisors Dr. Vipin Bhatnagar, Prof. Brajesh C. Choudhary, and Dr. Robert K. Plunkett for providing me guidance and encouragement throughout my Ph.D. years. Thank you for your advice and support over the last five years.

I am thankful to the conveners of the our group Justin Evans, Mike Kordosky, Donna Naples, Alex Sousa, and Jeff Hartnell who offered me all the help I needed for learning the MINOS software and performing my analysis tasks. I would like to thank Zeynep Isvan for teaching me the basics of performing an analysis and for always being there to help me debug my terrible programming mistakes. I would particularly like to thank Benton Pahlka, Joachim Kopp, and Teppei Katori for their help in making me understand the model on which the present thesis is based and in getting me started with the analysis. Special thanks to Joao A. B. Coehlo for all his help in the final stages of my work. Thanks also to Robert Hatcher and Art Kreymer who were always there to help me with the computing needs.

My visit to Fermilab would not have been possible without adequate financial support. Thanks to the India-Fermilab Neutrino Collaboration and Panjab University, Chandigarh for providing me the opportunity to work at Fermilab and supporting my stay.

I would like to thank all my friends and fellow students at Fermilab and Panjab University for making my stay at these two places enjoyable. Thanks also for helping me with all the official complexities of $\mathrm{PhD}$. It would have been enormously more difficult to do it alone. 
Finally, this would not have been possible without the support of my family. Many thanks to my parents and sister for their love and encouragement. This would not have been possible without you. 


\section{Abstract}

MINOS stands for Main Injector Neutrino Oscillation Search. It is a long baseline experiment located in the USA and is composed of two detectors. The Near Detector is at Fermilab, $1 \mathrm{~km}$ from the source of neutrinos. The Far Detector is in Minnesota at a distance of $735 \mathrm{~km}$ from the source. Both detectors are steel scintillator tracking calorimeters. MINOS searches for neutrino oscillations by comparing the neutrino energy spectrum at the Far Detector with that obtained from a prediction based on the spectrum at the Near Detector. The primary aim of MINOS is to measure the atmospheric oscillation parameters $\Delta m_{32}^{2}$ and $\theta_{23} . \mathcal{C P} \mathcal{T}$ symmetry requires that these parameters should be same for neutrinos and antineutrinos. Differences between neutrino and antineutrino oscillations would be an indication of new physics beyond the neutrino-Standard Model ( $\nu \mathrm{SM})$. Additionally, violation of Lorentz or $\mathcal{C P} \mathcal{T}$ symmetry could also give rise to oscillations different from that expected from the $\nu \mathrm{SM}$ predictions, such as neutrino to antineutrino transitions.

This thesis presents the measurements of antineutrino oscillation parameters using antineutrinos comprising a small $(7 \%)$ component of the $7.1 \times 10^{20}$ protons on target (POT) NuMI neutrino beam. The antineutrino component, being at a higher energy, is not able to constrain the parameters very precisely. Nevertheless, it helps provide additional bounds on the value of $\Delta m_{32}^{2}$ on top of the constraints provided by the analysis of antineutrino data obtained in a dedicated antineutrino running. Assuming $\sin ^{2}\left(2 \theta_{23}\right)=1$ the data excludes $\Delta m_{32}^{2}>4.49 \times 10^{-3} \mathrm{eV}^{2}$ at a $3 \sigma$ significance. The second analysis explores the possibility of Lorentz and $\mathcal{C P} \mathcal{T}$ violation in the neutrino sector by testing a model based on Standard 
Model Extension (SME). The model allows the possibility of neutrino to antineutrino transitions. The complete low energy neutrino and antineutrino datasets obtained in $10.56 \times 10^{20}$ POT exposure are fitted to the SME model. No indication of Lorentz or $\mathcal{C P} \mathcal{T}$ violation has been found. The data also provides world's first ever constraints on the SME parameters $\tilde{g}_{\mu \bar{\mu}}^{Z T}, \tilde{g}_{\tau \bar{\tau}}^{Z T},\left(c_{L}\right)_{\mu \mu}^{T T}$, and $\left(c_{L}\right)_{\tau \tau}^{T T}$. A study of the performance of the Far Detector in the six years of its running, with regard to the light level obtained from the PMTs, is also shown. The study shows that light levels from the Far Detector have been very stable over the years and the detectors will perform well in the MINOS+ era. 


\section{Publications/Conferences}

\section{Publications with Direct Contribution}

- P. Adamson et. al. (MINOS Collaboration), "Search for the disappearance of muon antineutrinos in the NuMI neutrino beam," Phys. Rev. D 84, 071103(R) (2011).

\section{MINOS Internal Notes with Direct Contribution}

- "Proposed Blessed Plots for the NuMuBar Forward Horn Current 7.1e20 Analysis," J. Evans, J. Hartnell, D. Naples, B. Pahlka, R. Sharma, M. Mathis, P. Vahle and Z. Isvan, MINOS Doc-7906 (2011).

- "Blessing Package for Attenuation Study in Far Detector," MINOS Doc-9064 (2012).

- "Position Paper for Light Level Studies," R. Sharma, MINOS Doc-9165 (2012).

- "Position Paper on the Neutrino-Antineutrino Oscillations in MINOS using 10.56e20 PoT Neutrino and Antineutrino Dataset," R. Sharma, MINOS Doc-9782 (2013).

\section{Talks and Posters Presented at Conferences}

- Presented a talk titled "Multivariate Analysis and Bayesian Neural Networks" at SERC SCHOOL in IIT, Bombay, February 9-27, 2009.

- Presented a talk titled "Comparison of Bayesian Neural Networks with TMVA" at the India-CMS meeting held at University of Delhi, Delhi, March 27-28, 2009. 
- Presented a talk titled "Measuring Antineutrino Oscillations in MINOS" at the APS Meeting, held at Anaheim, California, April 30-May 3, 2011.

- Presented a poster titled "Searching for Antineutrino Oscillations in a NuMI Neutrino Beam at MINOS" at the 44th Fermilab Annual Users' Meeting, June 1-2, 2011.

- Presented a talk titled "Study of Neutrino-Antineutrino Transitions in MINOS" at the APS Meeting, held at Atlanta, Georgia, March 31-April 3, 2012.

- Presented a poster titled "The Long Term Performance of the MINOS Calibration Procedure" by Sarah Phan-Budd (Argonne National Lab), Jeff de Jong (Oxford University), Luke Corwin (Indiana University), Mark Mathis (College of Willian and Mary), Richa Sharma (Fermilab), Nathaniel Tagg (Otterbein University) at the Neutrino 2012, held at Kyoto, Japan, June 3-9, 2012.

- Presented a poster titled "The Long Term Performance of the MINOS Calibration Procedure" at the 45th Fermilab Annual Users' Meeting, June 12-13, 2012.

- Presented a talk titled "Study of Neutrino-Antineutrino Transitions in MINOS" at the IIFC Meeting, held at Fermilab, November 26-27, 2012.

- Presented a talk titled "Study of Antineutrino Oscillations and Neutrino-Antineutrino Transitions in MINOS" at the IIFC Meeting, held at IIT Guwahati, February 7-10, 2013.

- Presented a poster titled "Searching for Neutrino to Antineutrino Oscillations in MINOS" at the 46th Fermilab Users' Meeting, held at Fermilab, June 12-13, 2013.

\section{MINOS Publications}

- P. Adamson et. al. (MINOS Collaboration), "Search for the disappearance of muon antineutrinos in the NuMI neutrino beam," Phys. Rev. D 84, 071103(R) (2011). 
- P. Adamson et. al. (MINOS Collaboration), "Improved search for muon-neutrino to electron-neutrino oscillations in MINOS," Phys. Rev. Lett. 107.181802.

- P. Adamson et. al. (MINOS Collaboration), "Active to Sterile Neutrino Mixing Limits from Neutral-Current Interactions in MINOS," Phys. Rev. Lett. 107, 011802 (2011).

- P. Adamson et. al. (MINOS Collaboration), "Search for Lorentz invariance and CPT violation with muon antineutrinos in the MINOS Near Detector," Phys. Rev. D. 85.031101.

- P. Adamson et. al. (MINOS Collaboration), "An improved measurement of muon antineutrino disappearance in MINOS," Phys. Rev. Lett. 208, 191801 (2012).

- P. Adamson et. al. (MINOS Collaboration), "Measurements of atmospheric neutrinos and antineutrinos in the MINOS Far Detector," Phys. Rev. D. 86, 052007 (2012).

- P. Adamson et. al. (MINOS Collaboration), "Comparisons of annual modulations in MINOS with the event rate modulation in CoGeNT," Phys. Rev. D. 87.032005 (2012).

- P. Adamson et. al. (MINOS Collaboration), "Electron neutrino and antineutrino appearance in the full MINOS data sample," Phys. Rev. Lett. 110, 171801 (2013).

- P. Adamson et. al. (MINOS Collaboration), "Measurement of Neutrino and Antineutrino Oscillation Parameters Using the Combined Beam and Atmospheric Data Sets from MINOS", Phys. Rev. Lett. 110, 251801 (2013).

- P. Adamson et. al. (MINOS Collaboration), "Combined Analysis of $\nu_{\mu}$ Disappearance and $\nu_{\mu} \rightarrow \nu_{e}$ Appearance in MINOS using Accelerator and Atmospheric Neutrinos," Phys. Rev. Lett. 112, 191801 (2014). 


\section{Contents}

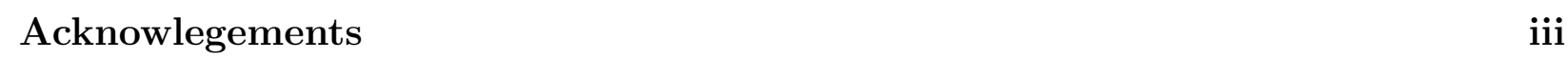

\begin{tabular}{lll}
\hline Abstract & v
\end{tabular}

Publications/Conferences vii

List of Tables $\quad$ xiv

\begin{tabular}{l|l}
\hline List of Figures & Xv
\end{tabular}

Preamble $\quad$ xxx

\begin{tabular}{|lll}
\hline 1 & The History and Theory of Neutrino Physics & 1
\end{tabular}

1.1 The Beginning . . . . . . . . . . . . . . . . . . . . . . 1

1.2 Discovery of the Neutrino . . . . . . . . . . . . . . . 3

1.3 The Weak Interaction $\ldots \ldots \ldots \ldots \ldots$

1.4 Three Neutrino Generations . . . . . . . . . . . . . . . . . . . . . . 5

1.5 Neutrinos in the Standard Model . . . . . . . . . . . . . . . . . . . . . 7

1.6 Neutrino Masses and Mixing . . . . . . . . . . . . . . . . . . . . . . . . 9

1.6 .1 Three Flavour Case . . . . . . . . . . . . . . . . . . . . . . 12

1.6 .2 Two Neutrino Mixing . . . . . . . . . . . . . . . . . . . . . . . . . . . 12

1.6 .3 Matter Effects . . . . . . . . . . . . . . . . . . . . . . . 14

1.7 Current Status of Measurements . . . . . . . . . . . . . . . . . . . . 16 
1.7 .1 Solar Neutrino Oscillations . . . . . . . . . . . . . . . . . . . . . 16

1.7 .2 Atmospheric Neutrino Oscillations $\ldots \ldots \ldots \ldots \ldots$

1.7 .3 Reactor Neutrino Oscillations $\ldots \ldots \ldots \ldots$. . . . . . . . . . 22

2 Lorentz and CPT Violation in the Neutrino Sector 28

2.1 Standard Model Extension . . . . . . . . . . . . . . . . . . . . . . . . . . . . 28

2.2 The Model used in this Thesis . . . . . . . . . . . . . . . . . . . . . . 30

2.3 Directional Dependence. . . . . . . . . . . . . . . . . . . . . . 34

$2.3 .1 \quad$ Effective Mixing Angle $\ldots \ldots \ldots \ldots$. . . . . . . . . . . 36

2.3 .2 Conditions for Resonance $\ldots \ldots \ldots \ldots \ldots$

2.4 Oscillation Probability . . . . . . . . . . . . . . . . . . . . 37

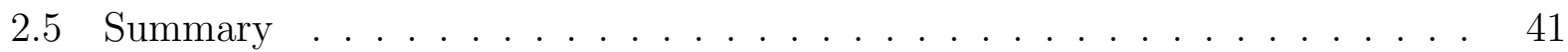

3 The MINOS Beam and Detectors 43

3.1 The NuMI Beam . . . . . . . . . . . . . . . . . . . . . . . . 43

3.2 The MINOS Detectors $\ldots \ldots \ldots \ldots \ldots$

$3.2 .1 \quad$ Near Detector . . . . . . . . . . . . . . . . . . . . 50

$3.2 .2 \quad$ Far Detector . . . . . . . . . . . . . . . . . . . . . 51

3.2 .3 Calibration Detector $\ldots \ldots \ldots \ldots \ldots \ldots \ldots$

3.3 The Detector Technology . . . . . . . . . . . . . . . . . . . . . . 54

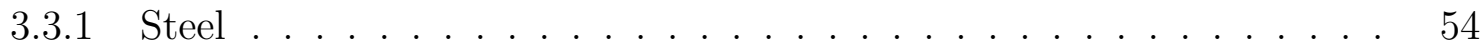

3.3 .2 Scintillator. . . . . . . . . . . . . . . . . . 54

3.3 .3 Photomultiplier Tubes $\ldots \ldots \ldots \ldots \ldots \ldots \ldots$

3.4 The Magnetic Field $\ldots \ldots \ldots \ldots \ldots$

3.5 Electronics and Data Acquisition $\ldots \ldots \ldots \ldots \ldots \ldots$

3.5 .1 Near Detector Front-End Electronics $\ldots \ldots \ldots$. . . . . . . . . 58

$3.5 .2 \quad$ Far Detector Front-End Electronics $\ldots \ldots \ldots \ldots$

3.5 .3 Data Acquisition . . . . . . . . . . . . . . . . . 63 
4 Calibration, Event Reconstruction and Monte-Carlo Simulation $\quad 67$

4.1 Calibration $\ldots \ldots \ldots \ldots \ldots$

$4.1 .1 \quad$ Calibration Procedure $\ldots \ldots \ldots$. . . . . . . . . . . . . . . . . . . . . . 68

$4.1 .2 \quad$ Absolute Energy Scale . . . . . . . . . . . . . . . . . . . . . . . . . 72

$4.2 \quad$ Event Reconstruction . . . . . . . . . . . . . . . . . . . . . . . . . . . . . . . . 74

$4.2 .1 \quad$ Event Topologies $\ldots \ldots \ldots \ldots$. . . . . . . . . . . . . . . 77

4.2 .2 Slice Formation $\ldots \ldots \ldots$. . . . . . . . . . . . . . . . . . . . . 77

4.2 .3 Track Reconstruction . . . . . . . . . . . . . . . . . . . . . . . . . . . . . . 78

4.2 .4 Cluster Formation and Shower Reconstruction . . . . . . . . . . . . . 80

4.2 .5 Event Formation . . . . . . . . . . . . . . . . . . . . . . . 82

4.3 Monte-Carlo simulation . . . . . . . . . . . . . . . . . . . . . . . . . . . . . . . . . 83

4.4 Analysis Dataset $\ldots \ldots \ldots \ldots$. . . . . . . . . . . . . . . . . . . 85

\begin{tabular}{lll}
\hline 5 & Antineutrinos in a Neutrino Beam & 87
\end{tabular}

5.1 Event Selection . . . . . . . . . . . . . . . . . . . . . . . . . 88

5.1 .1 Preselection . . . . . . . . . . . . . . . . . . 89

5.1 .2 Selection . . . . . . . . . . . . . . . . . . . . 89

5.2 Near Detector Spectrum . . . . . . . . . . . . . . . . . . . . . . . . . . . . . 91

5.3 Extrapolation (the Beam Matrix) . . . . . . . . . . . . . . . . . . . . . 96

5.4 Far Detector Data $\ldots \ldots \ldots$. . . . . . . . . . . . . . . . . . . . 102

5.5 Systematics . . . . . . . . . . . . . . . . . . . . . 104

5.5 .1 Track Energy Scale . . . . . . . . . . . . . . . . . . 105

5.5 .2 Shower Energy Scale . . . . . . . . . . . . . . . . . . 106

5.5 .3 Backgrounds . . . . . . . . . . . . . . . . 107

5.5 .4 Near to Far Normalisation . . . . . . . . . . . . . . . . . . . 107

5.5 .5 Cross-sections . . . . . . . . . . . . . . . . . . . 107

5.5 .6 Flux Modelling . . . . . . . . . . . . . . . . . . . . . . 108

5.5 .7 Downstream Events . . . . . . . . . . . . . . . . . . . . . . . . . 108 
5.5 .8 Effect on the Analyses . . . . . . . . . . . . . . . . . . . . . 109

5.6 Oscillation Analysis . . . . . . . . . . . . . . . . . . . . . . . . . . 109

6 Neutrino to Antineutrino Oscillations 115

$6.1 \quad$ Event Selection . . . . . . . . . . . . . . . . . . . . . . . . . . 118

6.1 .1 The Antineutrino Dataset . . . . . . . . . . . . . . . . . . . . . . . . 118

6.1 .2 The Neutrino Dataset $\ldots \ldots \ldots \ldots$. . . . . . . . . . . . . . 118

6.2 Far Detector Prediction $\ldots \ldots \ldots \ldots \ldots \ldots$

6.3 Generating Simulated Data . . . . . . . . . . . . . . . . 125

6.4 Cross-check of the extrapolation framework . . . . . . . . . . . . . . . . . 127

6.5 Fitting Procedure . . . . . . . . . . . . . . . . . . . . . . . . 128

6.6 Systematic Uncertainties . . . . . . . . . . . . . . . . . . . . . . . . 130

6.6 .1 Track Energy Scale . . . . . . . . . . . . . . . . . . . . . . . . . . . 131

6.6 .2 Shower Energy Scale . . . . . . . . . . . . . . . . . . . . 131

6.6 .3 Backgrounds . . . . . . . . . . . . . . . . . . . . . . . . 131

6.6 .4 Normalisation . . . . . . . . . . . . . . . . . . 132

6.6 .5 Cross-sections . . . . . . . . . . . . . . . . . . . . . . . 132

6.6 .6 Flux Modelling . . . . . . . . . . . . . . . . . . . . . . . . . . . . 132

6.6 .7 Downstream Events . . . . . . . . . . . . . . . . . . . . . 132

6.6 .8 Effect of Systematic Uncertainties . . . . . . . . . . . . . . . . . . . . 134

6.7 Including Systematics in Fitting . . . . . . . . . . . . . . . . . . . 136

6.8 Sensitivity $\ldots \ldots \ldots \ldots \ldots \ldots$

6.9 Results $\ldots \ldots \ldots \ldots \ldots$

\begin{tabular}{|lll}
\hline 7 & Summary and Outlook & 144
\end{tabular}

\begin{tabular}{|ll|}
\hline A Light Level Studies for the Far Detector & 147
\end{tabular}

A.1 Introduction . . . . . . . . . . . . . . . . . . . . 147

A.2 Average Pulse Height $\ldots \ldots \ldots \ldots$. . . . . . . . . . . . . . . . . . . . . . . . 147 
A.2.1 Strip Selection . . . . . . . . . . . . . . . . . . 147

A.2.2 Procedure . . . . . . . . . . . . . . . . . . . . . . . . . 148

A.3 Pulse Height Ratios . . . . . . . . . . . . . . . . . . . . . . . . . . . . . . . . 148

A.4 Attenuation Lengths . . . . . . . . . . . . . . . . . . . . . . . 150

A.5 Light level at the Centre of the Detector . . . . . . . . . . . . . . . . . 150

A.6 Summary . . . . . . . . . . . . . . . . . . . . . . . . . . . 152 


\section{List of Tables}

1.1 Particles of the standard model . . . . . . . . . . . . . . . . . . . . . 8

5.1 Components of the near-to-far normalisation systematic uncertainty. Table

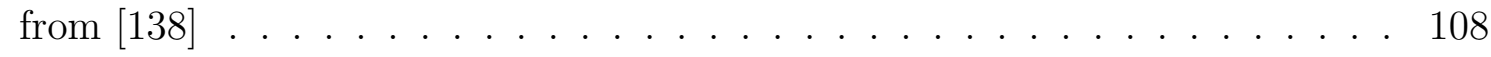

$6.1 \quad$ Best-fit values of the parameters $\Delta m^{2}, \sin ^{2} 2 \theta$, and $\left|\tilde{g}_{\mu \bar{\mu}}^{Z T}\right|$ obtained from the fit to simulated FD data generated at four different values of $\tilde{g}_{\mu \bar{\mu}}^{Z T}$. . . . . . . 129

6.2 Shifts in the measurements of the parameters $\tilde{g}_{\mu \bar{\mu}}^{Z T}$ or $\tilde{g}_{\tau \bar{\tau}}^{Z T}$, and $\left(c_{L}\right)_{\mu \mu}^{T T}$ or $\left(c_{L}\right)_{\tau \tau}^{T T}$ between the unshifted and shifted best-fit points for each systematic. Also shown is the change in the value of $-2 \log \lambda$ at best-fit. (Input: $\Delta m^{2}=2.32 \times$ $10^{-3} e V^{2}, \sin ^{2} 2 \theta=0.97, \tilde{g} \equiv\left(\tilde{g}_{\mu \bar{\mu}}^{Z T}\right.$ or $\left.\tilde{g}_{\tau \bar{\tau}}^{Z T}\right)=0, c \equiv\left(\left(c_{L}\right)_{\mu \mu}^{T T}\right.$ or $\left.\left.\left.\left(c_{L}\right)_{\tau \tau}^{T T}\right)=0\right)\right)$. The last row shows the size of the statistical uncertainty. . . . . . . . . . . . 135

6.3 Table showing the constraints on the individual SME parameters. . . . . . . 141

A.1 Change in light level over $6 \mathrm{~m}$. The change is calculated from the pulse height ratio at $3 m$ and $-3 m$ from the centre. 


\section{List of Figures}

1.1 Feynman diagrams of neutrino-quark interactions $\ldots \ldots \ldots$

1.2 Measurement of the hadron production cross section around the $Z^{0}$ mass resonance at LEP. The curves show the prediction for this cross section with 2 ,

3 , and 4 light active neutrino species. The results constrain $\mathrm{N}_{\nu}$ to $2.984 \pm 0.008$

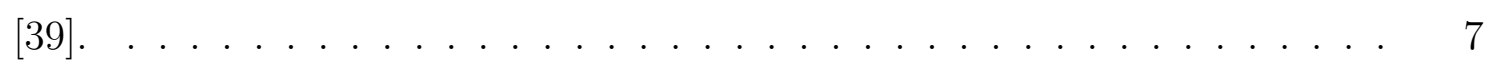

1.3 The solar neutrino spectrum predicted by the SSM [54]. The numbers associated with the neutrino sources show theoretical errors of the fluxes. (Figure source: http://www.sns.ias.edu/jnb/. . . . . . . . . . . . . . . . 17

1.4 Ratio of the measured $\bar{\nu}_{e}$ spectrum to the expectation for no-oscillation as a

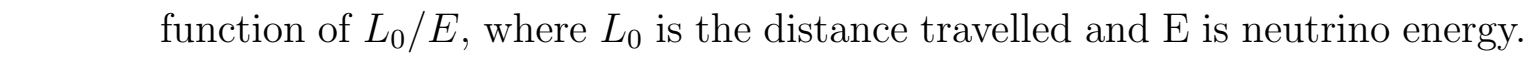
Also shown is the best fit for oscillation hypothesis. Figure from [61]. . . . . 19

1.5 Oscillation parameters allowed by the KamLAND and Solar experiments . . 20

$1.6 \quad$ Zenith angle distributions for e-like and $\mu$-like events with visible energy $<$ $1.33 \mathrm{GeV}($ sub-GeV) and > 1.33 GeV (multi-GeV). The dotted histograms show the non-oscillated Monte Carlo events, and the solid histograms show the best-fit expectations for $\nu_{\mu} \rightarrow \nu_{\tau}$ oscillations. Figure from [42]. . . . . . . 21

1.7 Atmospheric neutrino oscillation contours from MINOS and Super-Kamiokande 23 
1.8 Top: Measured prompt energy spectrum of the far hall compared with nooscillation prediction based on the measurements of the two near halls. Bottom: The ratio of measured and predicted no-oscillation spectra. The solid curve shows the expected ratio with oscillations, assuming $\sin ^{2} 2 \theta_{13}=0.089$.

The dashed line is the no-oscillation prediction. Figure from [74]. . . . . . .

1.9 Top: Measured prompt energy spectrum of the far detector compared with no-oscillation prediction based on the measurements of the near detector.

The backgrounds shown in the inset are subtracted from the far detector spectrum. Bottom: The ratio of measured spectrum at the far detector and the no-oscillation prediction. Figure from [75]. . . . . . . . . . . . . . . 26

1.10 The $68 \%$ and $90 \%$ confidence intervals for $\sin ^{2}\left(2 \theta_{13}\right)$ scanned over values of $\delta_{C P}$. The left plot assumes normal hierarchy and the right plot is for inverted hierarchy. Figure from [76]. . . . . . . . . . . . . . . . . . .

2.1 Co-ordinate system used for this analysis: a) the Sun-centered system, b) the Earth-centered system, c) the local polar coordinate system, d) the time zero is defined as the location of the experimental site at midnight near autumnal equinox. Figure from [95]. . . . . . . . . . . . . . . . . 34

2.2 The plot shows the effective mixing angle, $\theta_{C-o d d}$ or $\theta_{C-\text { even }}$ as a function of neutrino energy $E$. We have chosen $\Delta m^{2}=2.32 \times 10^{-3} \mathrm{eV}^{2}, \sin ^{2} 2 \theta=0.97$ (values taken from [97]), and four different values of $\tilde{g}_{\mu \bar{\mu}}^{Z T} \cdot \tilde{g}_{\mu \bar{\mu}}^{Z T} \cdot\left(c_{L}\right)_{\mu \mu}^{T T}$, and $\left(c_{L}\right)_{\tau \tau}^{T T}$ are assumed to be zero. The dotted vertical line denoting the energy threshold is drawn at $E=\sqrt{\Delta m^{2} / 1.6\left|\tilde{g}_{\mu \bar{\mu}}^{Z T}\right|} \mathrm{GeV}$. We can see the threshold decreases as the value of $\tilde{g}_{\mu \bar{\mu}}^{Z T}$ is increased. . . . . . . . . . . . . . . 40

2.3 Oscillation probabilities for $\nu_{\mu} \rightarrow \bar{\nu}_{\mu}$ and $\nu_{\mu} \rightarrow \bar{\nu}_{\tau} .\left(\tilde{g}_{\alpha \bar{\alpha}}^{Z T}=2 \times 10^{-22}, \Delta m^{2}=\right.$ $\left.2.32 \times 10^{-3} \mathrm{eV}^{2}, \sin ^{2} 2 \theta=0.97\right)$. 
2.4 Probabilities for $\nu_{\mu} \rightarrow \nu_{\mu}$ survival and $\nu_{\mu} \rightarrow \nu_{\tau}$ oscillation as a function of neutrino energy. The red line shows the oscillation probabilities using the new model with $\tilde{g}_{\alpha \bar{\alpha}}^{Z T}=2 \times 10^{-22}$ and the black line shows the oscillation probabilities using the standard two-flavour oscillations model $\left(\Delta m^{2}=2.32 \times\right.$ $10^{-3} \mathrm{eV}^{2}$ and $\sin ^{2} 2 \theta=0.97$ in both cases). . . . . . . . . . . . . . .

3.1 The layout of the MINOS Experiment showing the location of the Near and Far Detectors, and the distance between them.

3.2 Components of the NuMI beamline. Protons of $120 \mathrm{GeV}$ from FNAL Main Injector enter from the left. Figure from [101]. . . . . . . . . . . . . . . .

3.3 The $\mathrm{p}_{\mathrm{T}}$ vs. $\mathrm{p}_{\mathrm{Z}}$ distribution of the $\pi^{ \pm}$parents that produce neutrinos (left) and antineutrinos (right) at the ND when the beam is in low-energy FHC mode. 47

$3.4 \quad$ Near detector spectra of muon neutrinos and antineutrinos in FHC-mode. . $\quad 47$

$3.5 \quad$ Near detector spectra of muon neutrinos and antineutrinos in RHC-mode. . $\quad 48$

3.6 Three possible configurations of the NuMI beam (low, medium and high energy: LE, ME and HE). The relative target and focusing horn positions are shown on the left and the corresponding energy spectra on the right. . . . . 49

3.7 The MINOS Near (left) and Far (right) detectors. . . . . . . . . . . . . . . 49

3.8 A schematic of a MINOS detector showing the scintillator strips and the darker steel planes. . . . . . . . . . . . . . . . . . . . 50

3.9 The cross-section of a MINOS ND plane. The grey area is covered with scintillator in the partially instrumented planes, the entire plane being covered in the fully instrumented planes. The central diamond shows the position of the coil. The dark circle marks the centre of the neutrino beam. . . . . . . . 51

3.10 The arrangement of scintillator strips on the Near Detector planes. . . . . . 52 3.11 The arrangement of scintillator strips on the Far Detector planes. . . . . . . 52 
3.12 A CalDet subsection of 12 planes. The full detector was made of twelve such subsections. Detector planes are shown on the left and readout equipment on the right. . . . . . . . . . . . . . . . . . . . 53

3.13 Cross-section of a MINOS scintillator strip. . . . . . . . . . . . . . . . . . . 54

3.14 Schematic drawing of the scintillator system readout for a module. An edge of a detector plane is on the right side of the sketch, showing several strips extending out of a scintillator module and beyond the edge of the plane for clarity. The light produced in a strip travels out of the module in a WLS fiber, and is then carried by a clear optical fiber (assembled into a cable) to a MUX box where it is routed to a pixel of the PMT assembly. . . . . . . . . . 56

3.15 Cross-sectional view of one of the Far Detector supermodule coils. The larger diameter circles represent the copper cooling tubes and the smaller circles are the 190 turns of $1 / 0$ gauge stranded copper wire. . . . . . . . . . . . . 57

3.16 Magnetic field maps for a typical near (left) and far (right) detector planes. \begin{tabular}{|c}
\hline The grayscale indicates the magnetic field strength B as calculated by finite \\
\hline
\end{tabular} element analyses using 3-D models. The detector planes are shown looking upstream to the neutrino beam. . . . . . . . . . . . . . . . 58

3.17 Response of the QIE-chip based electronics: x-axis shows the amount of charge injected into the chip, while y-axis shows the ADC response for the different ranges. Only the first five out of the eight ranges are shown. . . . . . . . . . 60

3.18 Block diagram of a MENU module and a MINDER module. . . . . . . . . . . 61

3.19 Overview of the MINOS Near Detector readout electronics. . . . . . . . . . 62

3.20 Overview of the MINOS Far Detector readout electronics. . . . . . . . . . . 63

3.21 The architecture of the MINOS DAQ system for the Far Detector. For clarity, only one of the six PVIC input branches is completely illustrated. . . . . . . 
4.1 Stopping power of muons as a function of their momentum. The prediction by the Bethe-Bloch equation in polystyrene scintillator agrees very well with the MINOS FD data and the MINOS MC simulation. Both data and MC points have been normalised to the Bethe-Bloch calculation to give the expected stopping power at the minimum ionising point. Image from [105]. . . . . . . 71

4.2 Calorimetric response to pions and electrons at three momenta. The calorimetersignal scale is in arbitrary units. The data obtained from CalDet is compared to the simulation. Image from $[105] . \ldots \ldots \ldots \ldots$

4.3 A summary of the MINOS calibration procedure. Each step corresponds to a correction factor in Equation 4 4.1. The correction from raw pulse height (ADC) to SigLin applies the linearity calibration, L and drift correction D; from SigLin to SigCor the strip-to-strip calibration S; from SigCor to SigMap the attenuation correction $\mathrm{A}$ and finally the absolute energy scale calibration M to convert the raw signal into the standardised energy unit MEU. Taken from [105].

4.4 Response of the Far Detector in ADCs(left), in SigCors(middle) and in SigMaps(right), as a function of detector position, for U strips. . . . . . . . . . . . . . . . . . 74

4.5 Response of the Near Detector in ADCs(left), in SigCors(middle) and in SigMaps(right), as a function of detector position, for U strips. . . . . . . . . 75

4.6 Far (above) and Near (below) detector MEU as a function of time, as measured using cosmic data. Individual runs outside of the Run period boundaries were removed. Each point corresponds to one month's worth of data. Each point is normalised to the overall MEU for the whole period. . . . . . . . . . . . . 75

4.7 An example of a Near Detector snarl with several neutrino events distributed in space (left) and time (right). Figure from [112]. . . . . . . . . . . . . . 
4.8 The three event topologies relevant to the antineutrino analysis: $\mathrm{CC} \nu_{\mu}$ (left), $\mathrm{CC} \bar{\nu}_{\mu}$ (centre), and NC (right). The top row shows the Feynman diagram and the bottom row shows a representative simulated event in one view (i.e. only U planes). CC events are characterised by long muon tracks which curve in opposite directions for $\mathrm{CC} \nu_{\mu}$ and $\mathrm{CC} \bar{\nu}_{\mu}$. NC events do not have true muon tracks, but can have fake tracks which make them a background at low energy. The green points are hits with light levels below two photo-electrons and are not included in the analysis. . . . . . . . . . . . . . . . . . . . . . 78

4.9 Illustration depicting the use of the $\mathrm{kNN}$ technique for shower energy estimation. In this two-dimensional space, higher energy training events (represented by larger circles) correlate with larger values of the variables. The energy of the event marked by the star is estimated by examining its $\mathrm{k}$ (here 20) nearest neighbours, highlighted in red. The energy estimate is the mean of the true energies of these neighbouring events. . . . . . . . . . . . . . . . . 82

4.10 The accumulated POT as a function of time for all MINOS running. The dominant running mode is low energy neutrino production in the forward horn current (FHC) configuration, shown in green. The antineutrino production mode, using the reversed horn current (RHC) configuration is indicated in orange. The higher energy modes, at different target positions, is shown in red.

5.1 Contamination in the positive sample after preselection. The black line represents the total contamination (1-purity), the red line represents the fraction of events that are mis-identified $\nu_{\mu} \mathrm{CC}$ (wrong sign) and the blue line represents the fraction of events that are neutral currents. . . . . . . . . . . . . . . . 90 
5.2 Charge-sign selection variables $\frac{\frac{q}{p}}{\sigma\left(\frac{q}{p}\right)}$ and Relative Angle are shown for the ND in data (black points) and simulation (total in red, background in blue). The total systematic uncertainty on the Monte Carlo is represented by the shaded red bars. In each plot, all other selection cuts have been applied. . . . . . . . . 91

5.3 The three variables that form the PDFs in the $D p I D$ CC/NC separator: the track length, the fraction of the event energy in the track, and the mean energy deposited per plane are shown for the ND in data (black points) and simulation (total in red, background in cyan). The flux uncertainty on the Monte Carlo is represented by the shaded red bars and the background systematic uncertainty is represented by the shaded blue bars. . . . . . . . . . . . . . . . . . . . . . 92

5.4 CC/NC separation parameter $(D p I D)$ is shown for the ND in data (black points) and total Monte Carlo with flux uncertainty (red line and shaded bars). Also shown is the total background in blue. The CC/NC separator has some power to reject the wrong-sign background due to the higher average inelasticity $(y)$ of neutrinos compared to antineutrinos. All other cuts have been applied. . . . . . . . . . . . . . . . . . . . . . . 93

5.5 This figure shows the contamination in the sample of events with positive reconstructed charge. The dashed and solid lines respectively show the contamination before and after the selection cuts. NC background is in blue and wrong-sign background is in red. . . . . . . . . . . . . . . . . . . 93

5.6 The track energy (left) and shower energy (right) distributions shown for the ND; data as black points and simulation (total in red, background in blue). The total systematic uncertainty on the Monte Carlo is represented by the shaded red bars. . . . . . . . . . . . . . . . . . . . . . . . 94

5.7 The selected $\bar{\nu}_{\mu}$ energy spectrum is shown for the ND; data is shown as black points, background as blue and tuned MC in red. The total systematic uncertainty on the Monte Carlo is represented by the shaded red bars. . . . . . 94 
5.8 The selected $\bar{\nu}_{\mu}$ energy spectrum is shown for the ND; data (black points), untuned simulation (blue line), and simulation with tuned hadron production (red line). The ratios of the data to the two simulations are shown below this figure. . . . . . . . . . . . . . . . . . . . . . 96

5.9 Top plots show the track vertex in X direction (left) and in Y direction (right) in meters. Bottom plots show the radius squared distribution of track vertex (left) and track vertex in $\mathrm{Z}$ direction (right) in units of number of planes. Black dots represent the ND data and the red curve represents Monte Carlo. The Monte Carlo is area normalised to data to compare the shapes of the distributions independent of normalisation. . . . . . . . . . . . . . . . . . 97

5.10 Diagram of the neutrino parents in the NuMI decay pipe. A parent will typically have a wide range of neutrino decay angles that reach the Near Detector and a very narrow range that will reach the Far Detector. . . . . . 98

5.11 Simulated true neutrino energy spectra in the near (left) and and far (right) detectors. The coloured regions on the left and right spectra show the neutrino energy distributions coming from the same neutrino parents. . . . . . . . . . 99

5.12 The beam matrix for $\bar{\nu}_{\mu}$ 's in the FHC beam. Each cell relates a FD energy bin to a ND one. The content of each cell represents the mean number of $\bar{\nu}_{\mu}$ events expected in the FD for one event in the ND. This distribution is treated as a matrix to relate the energies measured in the ND to those expected in the FD. . . . . . . . . . . . . . . . . . . . . . . 100

5.13 Flowchart showing the steps involved in the extrapolation of a Near Detector energy spectrum to a Far Detector prediction. . . . . . . . . . . . . . . . . . 101

5.14 Beam matrix extrapolation procedure for antineutrinos, starting from the Far Detector flux calculated using Fig. 5.13 . Figure from [136]. 
5.15 Left: the raw FD Monte Carlo (black) and the prediction obtained by applying beam matrix extrapolation to the ND Monte Carlo (red) for the CC $\bar{\nu}_{\mu}$ spectra. Right: the corresponding ratios of the prediction divided by the FD Monte Carlo . . . . . . . . . . . . . . . . . . . . 102

5.16 Efficiency and Purity at the FD before and after selection cuts. . . . . . . . . 103

$5.17 \mathrm{CC} / \mathrm{NC}$ separation parameter $(D p I D)$ is shown for the FD; data (black points), oscillated simulation (solid red line) and unoscillated simulation (dashed red line). All other selection cuts have been applied. . . . . . . . . . . . . . . . . 104

5.18 Charge-sign selection variable $\frac{\frac{q}{p}}{\sigma\left(\frac{q}{p}\right)}$ is shown for the FD; data (black points), oscillated simulation (solid red line) and unoscillated simulation (dashed red line). All other selection cuts have been applied. . . . . . . . . . . . . . . . . 104

5.19 Charge-sign selection variable - RelativeAngle- $\pi$ - is shown for the FD; data (black points), oscillated simulation (solid red line) and unoscillated simulation (dashed red line). All other selection cuts have been applied. . . . . . . 105

5.20 Selected antineutrino event vertex (left) and end (right) positions for the FD as a function of $x$-coordinates . . . . . . . . . . . . . . . . . . 105

5.21 Selected antineutrino event vertex (left) and end (right) positions in the FD as a function of $y$-coordinates. . . . . . . . . . . . . . . . 106

5.22 FD systematic error band constructed from all systematic uncertainties summed in quadratue. . . . . . . . . . . . . . . . . . . . . . 110

5.23 Far Detector $\bar{\nu}_{\mu}$ data spectrum (dots) compared to predictions with no oscillations (dashed red histogram) and with best-fit oscillation parameters. Error bars on the data points represent the statistical errors and the band around of the histogram with best-fit prediction represents the total systematic uncertainty. Total background in the best-fit prediction is also displayed (gray shaded histogram) . . . . . . . . . . . . . . . . . . . . 112 
5.24 (Top) The 90\% antineutrino oscillation contours from FHC running, overlaid with the $2010 \mathrm{CC} \nu_{\mu}$ result and the $2010 \mathrm{RHC} \bar{\nu}_{\mu}$ result. The contours are determined using the Feldman-Cousins method. The best fit is at $\left|\Delta \bar{m}_{a t m}^{2}\right|=$ $18 \times 10^{-3} \mathrm{eV}^{2}$ and $\sin ^{2}\left(2 \bar{\theta}_{23}\right)=0.25$. The hatched area indicates the excluded region. (Bottom) One-dimensional fit of $\Delta \bar{m}^{2}$ at maximal mixing, showing the values excluded by this result. At maximal mixing, we exclude $\Delta \bar{m}^{2}>$ $4.49 \times 10^{-3} \mathrm{eV}^{2}$ at a $3 \sigma$ significance. . . . . . . . . . . . . . . . . . . 114

6.1 Distributions of the four variables used as input to the primary CC selection. Monte Carlo prediction is shown in red, with the shaded portion showing the systematic error associated with the beam flux. The expected neutral-current distribution is shown in blue. The black points show the distributions in the ND data. The data and Monte Carlo show good agreement. . . . . . . . . . 120

6.2 Output of $k \mathrm{NN}$ formed from variables in Fig. 6.1 . The data and Monte Carlo show good agreement. . . . . . . . . . . . . . . . . . . . . 121

6.3 Distributions of the input variables to the auxiliary CC selection. Conventions are as given in Fig. 6.1. . . . . . . . . . . . . . . . . . . . . . . . . 122

6.4 Output of the kNN formed from variables in Fig. 6.3 . The data and Monte Carlo show good agreement.

6.5 Efficiency (blue) and contamination (red) achieved by the charged-current selection at the far detector as a function of reconstructed energy. Above 1 GeV very high efficiency and very low contamination is obtained. . . . . . . 123

6.6 Steps to obtain the FD $\bar{\nu}_{\mu}$ prediction in reconstructed energy. The backgrounds: $\bar{\nu}_{\tau}$, 'wrong-sign' $\nu_{\mu}$ 's and NC backgrounds, are also added after applying oscillation weights appropriately.

6.7 Steps to obtain the FD $\nu_{\mu}$ prediction in reconstructed energy. The backgrounds: $\nu_{\tau}$, 'wrong-sign' $\bar{\nu}_{\mu}$ 's and NC backgrounds, are also added after applying oscillation weights appropriately. . . . . . . . . . . . . . . . . . 125 
$6.8 \quad$ FD $\bar{\nu}_{\mu}$ prediction with $\Delta m^{2}=2.32 \times 10^{-3} \mathrm{eV}^{2}, \sin ^{2} 2 \theta=0.97$, and different values of $\tilde{g}_{\alpha \bar{\alpha}}^{Z T}$, where $\alpha$ can be $\mu$ or $\tau$. As the value of $\mathrm{g} \equiv \tilde{g}_{\alpha \bar{\alpha}}^{Z T}$ increases, a peak is seen in the FD predicted spectrum due to lowering of the threshold for transitions. . . . . . . . . . . . . . . . . . . . . . 126

$6.9 \quad$ FD $\nu_{\mu}$ prediction with $\Delta m^{2}=2.32 \times 10^{-3} \mathrm{eV}^{2}, \sin ^{2} 2 \theta=0.97$, and different values of $\mathrm{g} \equiv \tilde{g}_{\alpha \bar{\alpha}}^{Z T}$.

$6.10 \bar{\nu}_{\mu}$ spectra showing agreement between FD prediction and simulated data. The data and prediction are produced with $\Delta m^{2}=2.32 \times 10^{-3} \mathrm{eV}^{2}, \sin ^{2} 2 \theta=0.97$, and different values of $\mathrm{g} \equiv \tilde{g}_{\mu \bar{\mu}}^{Z T}$.

6.11 One-dimensional $-2 \Delta \log \lambda$ distribution for the parameter $\tilde{g}_{\mu \bar{\mu}}^{Z T}$ obtained by fitting simulated data generated at $\tilde{g}_{\mu \bar{\mu}}^{Z T}=0,2 \times 10^{-24}, 2 \times 10^{-23}$, and $2 \times 10^{-22}$. The dashed lines show the $1-\sigma, 2-\sigma$, and3- $\sigma$ limit. The minimum value of $2 \Delta \log \lambda$ corresponds to the value of $\tilde{g}_{\mu \bar{\mu}}^{Z T}$ at which the simulated data was generated. . . . . . . . . . . . . . . . . . 130

6.12 Comparison of the decay pipe component of the antineutrino flux in an FHC beam obtained with different physical models. . . . . . . . . . . . . . . . 133

6.13 Ratio of the decay pipe component obtained with different models to that obtained from FLUGG.

6.14 1-D $\log$ (likelihood) plots showing the effect of the largest three systematics on $\mathrm{g} \equiv \tilde{g}_{\mu \bar{\mu}}^{Z T}$ when input $\tilde{g}_{\mu \bar{\mu}}^{Z T}=0$. All other parameters are marginalised.

6.15 Error bands used in the modelling of systematic uncertainties for the neutrinos (left - top and bottom) and antineutrinos (right - top and bottom). The smoothed distributions are interpolated linearly to obtain a continuous function $\mathrm{w}(\mathrm{E})$ that is applied to shift the FD predictions. 
6.16 1-dimensional $-2 \Delta \log \lambda$ distributions showing the fit to the parameters $\left|\tilde{g}_{\alpha \bar{\alpha}}^{Z T}\right|$, $\left(c_{L}\right)_{\mu \mu}^{T T}$, and $\left(c_{L}\right)_{\tau \tau}^{T T}$ for simulated data generated at $\tilde{g}_{\mu \bar{\mu}}^{Z T}=\tilde{g}_{\tau \bar{\tau}}^{Z T}=\left(c_{L}\right)_{\mu \mu}^{T T}=\left(c_{L}\right)_{\tau \tau}^{T T}=0$. The dashed lines show the $1-\sigma, 2-\sigma$, and $3-\sigma$ sensitivities for the parameters. The statistics-only sensitivity is shown in red and that with the systematics included in the fit is shown in black. . . . . . . . . . . . . . . . . . . . . . 139

6.17 FD antineutrino spectrum expected at the 3- $\sigma$ threshold of sensitivity to $g$ and c parameters, where $\mathrm{g} \equiv \tilde{g}_{\alpha \bar{\alpha}}^{Z T}$ and $\mathrm{c} \equiv\left(c_{L}\right)_{\mu \mu}^{T T}$ or $\left.\left(c_{L}\right)_{\tau \tau}^{T T}\right)$. The black dots show the spectrum when both $g$ and c-type SME coefficients are zero. The red histogram shows the FD spectrum predicted at $\tilde{g}_{\alpha \bar{\alpha}}^{Z T}=3.4 \times 10^{-23}$ and the blue histogram shows the FD prediction at $\left(c_{L}\right)_{\mu \mu}^{T T}=6 \times 10^{-23}$. . . . . . . . 140

6.18 1-dimensional $2 \Delta \log \lambda$ distributions showing the fit to the parameters $\left|\tilde{g}_{\alpha \bar{\alpha}}^{Z T}\right|$, $\left(c_{L}\right)_{\mu \mu}^{T T}$, and $\left(c_{L}\right)_{\tau \tau}^{T T}$. The dashed lines correspond to 1- $\sigma, 2-\sigma$ and 3- $\sigma$ confidence limits. . . . . . . . . . . . . . . . . . . . . . . . 141

6.192 -dimensional $-2 \Delta \log \lambda$ distribution showing the fit to the parameters $\mid \tilde{g}_{\mu \bar{\mu}}^{Z T}-$ $\tilde{g}_{\tau \bar{\tau}}^{Z T} \mid$ and $\left(\left(c_{L}\right)_{\mu \mu}^{T T}-\left(c_{L}\right)_{\tau \tau}^{T T}\right)$. The blue, green and red contours show the 1- $\sigma$,

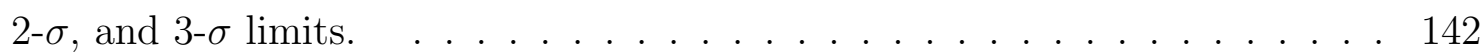

6.20 Far Detector distributions of selected antineutrino (left) and neutrino (right) events. Black dots represent data, the dashed histogram shows the prediction in the absence of oscillations, the dotted-dashed histogram shows the prediction at the values obtained in a fit to 2-flavour oscillation model, and the solid histogram shows the prediction at the values obtained in our fit to the SME model. The prediction obtained from a two flavour fit is very similar to that obtained from the SME fit. . . . . . . . . . . . . . . . . . . . . . . . 143

7.1 The $68 \%$ and $90 \%$ confidence limits for the parameters $\left|\Delta m_{32}^{2}\right|$ and $\sin ^{2} \theta_{23}$. Contours for both normal and inverted hierarchy are shown, and the $-\Delta \log \mathrm{L}$ surface is calculated relative to the overall best-fit point. A marginal preference for inverted hierarchy and lower octant is shown. . . . . . . . . . . . . . 145 
A.1 Average light output as a function of distance from the readout end for the year 2004. The light output from east and west ends for U-view and V-view strips have been combined. . . . . . . . . . . . . . . . . . . . . . . . . . . . . 149

A.2 Average light output as a function of distance from the readout end for the years 2007(left) and 2010. The light output from east and west ends for U-view and V-view strips have been combined. . . . . . . . . . . . . . . . . . . . . . 149

A.3 Ratio of average light output in the year 2010 to that in 2004 as a function of distance from the readout end. . . . . . . . . . . . . . . . . . . . . . . 150

A.4 Average light output as a function of distance from the readout end for the year 2004. The light output is fitted to a function of the form $A e^{-k_{1} x}+B e^{-k_{2} x}$. The fit parameters are $A=250.87, B=30.18, k_{1}=0.12 \times 10^{-2}, k_{2}=0.45$. After removing the region between $-2 \mathrm{~m}$ and $2 \mathrm{~m}$ the reduced fit parameters are $A=294.09, B=8.27 \times 10^{-12}, k_{1}=1.606 \times 10^{-1}, k_{2}=3.239 \times 10^{-1} \ldots . \quad 151$

A.5 Average light output as a function of distance from the readout end for the year 2007. The light output is fitted to a function of the form $A e^{-k_{1} x}+B e^{-k_{2} x}$. The fit parameters are $A=230.39, B=54.69, k_{1}=0.10 \times 10^{-2}, k_{2}=0.385$. After removing the region between $-2 \mathrm{~m}$ and $2 \mathrm{~m}$ the reduced fit parameters are $A=300.31, B=0.42 \times 10^{-12}, k_{1}=1.592 \times 10^{-1}, k_{2}=3.852 \times 10^{-1}$.

A.6 Average light output as a function of distance from the readout end for the year 2010. The light output is fitted to a function of the form $A e^{-k_{1} x}+B e^{-k_{2} x}$. The fit parameters are $A=226.06, B=62.85, k_{1}=8.9 \times 10^{-2}, k_{2}=0.387$. After removing the region between $-2 \mathrm{~m}$ and $2 \mathrm{~m}$ the reduced fit parameters are $A=307.49, B=85.8 \times 10^{-12}, k_{1}=1.577 \times 10^{-1}, k_{2}=3.066 \times 10^{-1} \ldots$. 153 
A.7 Ratio of average light output in the year 2010 to that in 2004 as a function of distance from the centre of the strips. (Left) The light output from the east and the west ends for strips in U-view have been combined, (Right) The light output from the east and the west ends for strips in U-view have been combined. . . . . . . . . . . . . . . . . . 153

A.8 Ratio of average light output in 2010 and 2004 as a function of distance from the centre of strips. The output from east and west readout ends of strips in both U-view and V-view have been combined. . . . . . . . . . . . . . . . . . 154

A.9 Ratio of light output in 2010 to 2004 as a function of radial distance from the centre of the detector. The light level at the centre changes by $2.6 \%$ w.r.t the average. . . . . . . . . . . . . . . . . . . 154 


\section{Preamble}

Neutrinos have been one of the most mysterious particles despite their abundance in the universe. When originally proposed [1], neutrinos were believed to be massless particles. But thanks to the compelling evidence that neutrinos can change flavour (oscillate), we now know that they have non-zero masses [2]. This is already an evidence for physics beyond the Standard Model (SM). Much is still unknown about neutrinos and we need to fully understand their properties. One way to unfold their properties is to study the relationship of the neutrino and its antiparticle. This thesis looks at the oscillation properties of antineutrinos. Any difference in the properties of neutrinos and antineutrinos would be a signature of physics not predicted by the SM. It could be a sign of new particle or new interactions with matter. It may even indicate a violation of one of the most fundamental symmetries of quantum field theory: Lorentz and $\mathcal{C P} \mathcal{T}$ invariance.

Chapter 1 of this thesis gives a brief introduction to the history of neutrino physics, discusses the theory of neutrino oscillations, and finally gives a summary of results obtained from various experiments to date.

It is interesting to see whether we can fit the MINOS data using an alternative model. Chapter 2 discusses an alternative model based on Standard-Model Extension (SME) which is formed by adding additional terms in the SM Lagrangian which allow Lorentz and $\mathcal{C P} \mathcal{T}$ violation. A feature of this model is that it allows neutrino-antineutrino transitions (which are forbidden in the SM). A brief description of the model and derivation of the oscillation probabilities is given, followed by a discussion of the expected signal. 
The MINOS experiment, which obtains an intense muon neutrino beam from the Neutrinos at the Main Injector (NuMI) beamline at Fermilab, is ideal for studying atmospheric oscillation parameters. Furthermore, since it is magnetised it can distinguish between neutrinos and antineutrinos from the muon tracks formed from their charged-current interactions in the detector. Chapter 3 gives the details of the design of the MINOS detectors and the NuMI beam.

The first step in any analysis is the reconstruction of neutrino and antineutrino events. A precise measurement of the neutrino oscillation parameters requires an accurate measurement of neutrino energies, which is done through a chain of calibrations. The details of the process of event reconstruction and detector calibration are given in Chapter 4. This chapter also describes the method for Monte Carlo simulation which is important for understanding and interpreting the probabilistic processes of particle physics from the limited data statistics.

Chapter 5 and 6 present the antineutrino and neutrino-antineutrino oscillation analyses, respectively, and report the findings.

Finally Chapter 7 summarises the results of this thesis and briefly discusses the future prospects of MINOS and for the field in general. 


\section{Chapter 1}

\section{The History and Theory of Neutrino Physics}

\subsection{The Beginning}

When the phenomenon of radioactivity was discovered in 1892 , the $\beta$-decay process was understood to be the process where only an electron is emitted from the radioactive nucleus. In the studies performed by Otto Hahn and Lise Meitner [3] the electron was thought to be emitted at a fixed energy corresponding to the change in binding energy of the nucleus. But after further experimentation, in 1911, they found that the electrons had an underlying continuous spectrum [4]. In 1914, James Chadwick made the discovery that the beta decay spectrum is continuous, and not monoenergetic [5]. It seemed to violate the conservation laws and therefore it was troubling to the physicists at the time. One explanation for the continuous spectrum in the early 1920s was that the electron lost varying fractions of energy while travelling through the medium containing the radioactive nuclei. In 1927, Ellis and Wooster performed experiments [6] to detect this missing energy in a calorimeter, but failed to detect it, thereby disproving the above hypothesis.

In 1930, Wolfgang Pauli wrote an open letter [1] to a conference in which he proposed "a desperate remedy to save the exchange theorem of statistics and the law of conservation 
of energy." He proposed that a new particle with no electrical charge and spin $\frac{1}{2}$ was being emitted along with the electron during $\beta$-decay. He named the particle 'neutron'. This particle was required to have a mass less than $1 \%$ of the proton mass. The continuous $\beta$-decay spectrum would then be explained by assuming that in addition to an electron, a 'neutron' is also emitted such that the sum of energies of the neutron and electron is constant. Later, the "real neutron" was discovered by Chadwick in 1932 [7], but it was realised to be the particle in the nucleus which emitted the electron and the neutrino in the process of $\beta$-decay [8, 9, 10, 11]. Around the same time, Francis Perrin [12] and Enrico Fermi [13, 14] proposed the idea of the neutrino as a massless particle created during the process of $\beta$-decay.

In 1933, Enrico Fermi developed his successful theory of $\beta$-decay, constructed using the neutrino, electron, neutron and the proton, analogous to the earlier theory of electromagnetic interactions. With this a new field of theoretical physics came into existence - the field of Weak Interactions. Fermi adopted the proton-neutron theory of the atom and proposed the name neutrino for the Pauli's neutron, meaning "the little neutral one". The theory requires the neutrino to carry energy, linear momentum, as well as angular momentum or 'spin' from the decaying nuclei. It is illustrated by the decay of the free neutron:

$$
n \rightarrow p+e^{-}+\nu
$$

This theory was also generalised by Gamow and Teller in 1936 [15], and it was able to describe all $\beta$-decay data that had been collected. In his letter, one of Pauli's major concern was that the neutrino should already have been seen if it existed. This was addressed by Hans Bethe and Rudolf Peierls in 1934, who showed that the cross section for the neutrino to interact with a nucleus is less than $10^{-44} \mathrm{~cm}^{2}$, corresponding to a penetrating power of $10^{16} \mathrm{~km}$ in ordinary matter [16]. Because of the small cross section it was considered an undetectable particle for many years. 


\subsection{Discovery of the Neutrino}

In 1953, an experiment was set up by Clyde Cowan and Fred Reines at the Hanford reactor site [17, 18, 19, 20, 21], where they obtained a $2 \sigma$ signal for the existence of neutrinos. Since this signal was not conclusive, in 1956, they performed another experiment to make a direct observation of the neutrino [22]. Their detector was set up close to the Savannah River nuclear reactor which gave a large flux of neutrinos. These are now known to be electron antineutrinos. The interaction target in the detector was 200 litres of water with $40 \mathrm{~kg}$ of $\mathrm{CdCl}_{2}$ dissolved in it. It was sandwiched between three liquid scintillator layers which were instrumented with 110,5 " photomultiplier tubes each. The antineutrinos were detected through inverse $\beta$-decay:

$$
\bar{\nu}_{e}+p \rightarrow e^{+}+n
$$

A distinct pattern of activity in the detector was required in order to confirm the presence of neutrinos: 1) Annihilation of a positron with an electron in water which would produce two $511 \mathrm{keV}$ photons moving in opposite directions and picked up in coincidence in the scintillator, 2) Capture of the neutron by the dissolved cadmium, emitting a photon or many photons that would be picked up in the scintillator within $5 \mu$ s of the initial photon pair from $\mathrm{e}^{+} \mathrm{e}^{-}$annihilation. After months of data collection they had accumulated data of about three observed neutrinos per hour. To confirm that they were actually observing neutrinos they shut down the reactor and showed that the rate of these interactions dropped to zero. Reines was honoured by the Nobel Prize in 1995 for his work on neutrino physics (Clyde Cowen died in 1974).

\subsection{The Weak Interaction}

Using the neutrino hypothesis of Pauli, Fermi developed the theory of weak interaction by analogy with the theory of electromagnetic interaction. If we denote the fermion fields 
for the neutron, the proton, the electron and the neutrino by $\mathbf{u}_{i}$, where $i=n, p, e$, and $\nu_{e}$, the $\beta$-decay matrix element for the four-Fermi interaction can be written as

$$
M=G\left(\bar{u}_{n} \gamma^{\mu} u_{p}\right)\left(\bar{u}_{\nu} \gamma_{\mu} u_{e}\right)
$$

where $G$ is the coupling constant and $\gamma_{\mu}$ are the Dirac gamma matrices. However, over the years some modifications of the original form of the four-Fermi interaction were required. Firstly, the fundamental fields entering the Hamiltonian are fields describing quarks, rather than hadronic fields like the proton or the neutron. Secondly, Pauli had assumed parity conservation and, therefore, the interaction had the vector-vector form. With the discovery of parity violation [23] it was necessary to include an axial component of the form $\left(\gamma_{\mu} \gamma_{5}\right)$ in the weak current. The vector-axial (V-A) form of the currents was later noted by Marshak and Sudarshan, and by Feynman and Gell-Mann [24, 25]. Thus the $\beta$-decay matrix element takes the form

$$
M=G\left(\bar{u}_{n} \gamma^{\mu}\left(1-\gamma_{5}\right) u_{p}\right)\left(\bar{u}_{\nu} \gamma_{\mu}\left(1-\gamma_{5}\right) u_{e}\right)
$$

Although the four-Fermi interaction in Eq. 1.3 provides an excellent description of the low energy weak interaction phenomena, it has certain difficulties as a field theory. For instance, the higher order corrections to any lowest order weak process are divergent. Besides this, even at the lowest order the theory predicts the total cross section $\sigma(\nu n) \propto E_{\nu}^{2}$. With increasing energy this cross section grows without limit, and hence, violates unitarity at around $300 \mathrm{GeV}$ [26] (first noticed by Heisenberg in 1936 [27]). The basic problem was due to the fact that the theory is non-renormalisable. The first renormalisable theory of weak interactions was given by Sheldon Glashow [28], Steven Weinberg [29] and Abdus Salam [30]. This theory predicted a neutral current component to the weak interaction, and masses for 
the exchange bosons ( $\mathrm{W}$ and $\mathrm{Z}$ ).

The weak neutral currents were first observed in 1973 in the Gargamelle bubble chamber in the $\nu_{\mu}$ e scattering [31, 32. The masses of the exchange bosons, $\mathrm{W}$ and $\mathrm{Z}$, were first measured in the UA1 and UA2 experiments on the $p \bar{p}$ collider at CERN [33, 34, 35, 36]. The Feynman diagrams showing charge and neutral current interactions of neutrinos are shown in Fig. 1.1.
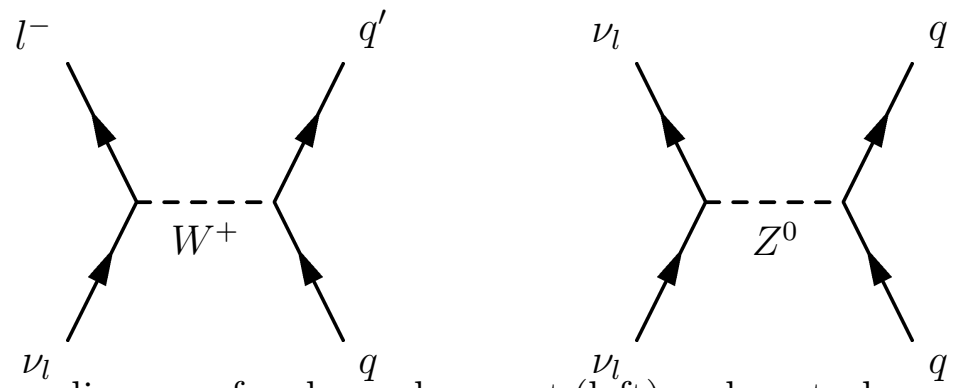

Figure 1.1: Feynman diagrams for charged current (left) and neutral current (right) neutrinoquark interactions.

\subsection{Three Neutrino Generations}

The first evidence for the existence of more than one neutrino type or 'flavour' came in 1962 from an experiment performed by Leon Lederman, Melvin Schwartz, and Jack Steinberger at the Brookhaven AGS [37]. The neutrinos for this study were generated in the first accelerator neutrino beam. Protons were accelerated to $15 \mathrm{GeV}$ and made to strike a beryllium target. The resulting $\pi^{ \pm}$were allowed to decay in a $21 \mathrm{~m}$ channel where most of the neutrinos are produced according to the following reaction:

$$
\begin{aligned}
& \pi^{+} \rightarrow \mu^{+}+\nu \\
& \pi^{-} \rightarrow \mu^{-}+\bar{\nu} .
\end{aligned}
$$

There was a $13.5 \mathrm{~m}$ iron absorber at the end of the decay channel followed by a 10 ton aluminium spark chamber to observe neutrino interactions. The charged particles were stopped by the absorber and the neutrinos passed to the detector. It was expected that if 
there were only one kind of neutrino, then neutrino interactions would produce muons and electrons in equal abundance. However only muons were observed in the experiment:

$$
\begin{aligned}
& \nu_{\mu}+N \rightarrow \mu^{-}+X \\
& \nu_{\mu}+N \nrightarrow e^{-}+X,
\end{aligned}
$$

It showed that there were at least two families of neutrinos - one coupling to the electron and the other to the muon. Lederman, Schwartz, and Steinberg received the Nobel Prize for this discovery in 1988.

The first evidence that a third generation of leptons, the $\tau$ lepton exists came from SLAC in 1975 [38]. With this discovery the possibility of existence of another type of neutrino, the $\tau$ neutrino also arose. The first indirect evidence for a third type of neutrino came from the experiments ALEPH, DELPHI, L3 and OPAL on the Large Electron Positron Collider (LEP) [39]. These experiments were designed to precisely measure the $\mathrm{Z}$ boson cross section. If the total width of the $\mathrm{Z}$ resonance is compared to the width from its decays to visible particles it yields the 'invisible width', which is the width from its decays to particles that cannot be observed in the detector, namely $Z^{0} \rightarrow \nu \bar{\nu}$. This makes the $\mathrm{Z}$ boson width very sensitive to the number of neutrino flavours. Fits to the LEP data assuming equal couplings strengths of the $\mathrm{Z}$ to all neutrino types yields the number of neutrino types to be $2.984 \pm 0.008$. Fig. 1.2 shows the predictions at different values for the number of neutrino generations.

The first direct evidence for $\tau$ neutrinos came from the DONUT collaboration at Fermilab in 2001 [40]. They created a $\nu_{\tau}$ enriched beam by colliding an $800 \mathrm{GeV}$ proton beam on a thick tungsten target. The target absorbed most of the pions and hadrons leaving a beam rich in $D_{s}$ mesons, which decay to $\tau \nu_{\tau}$ with a branching fraction of $6 \%$. The neutrino beam, obtained after removing all non-neutrino particles, is passed through a detector composed of steel and emulsion planes. The charged-current interactions, $\nu_{\tau}+N \rightarrow \tau^{-}+X$ are used to identify the tau neutrino. The tau decays in the emulsion after a short track, which is seen 


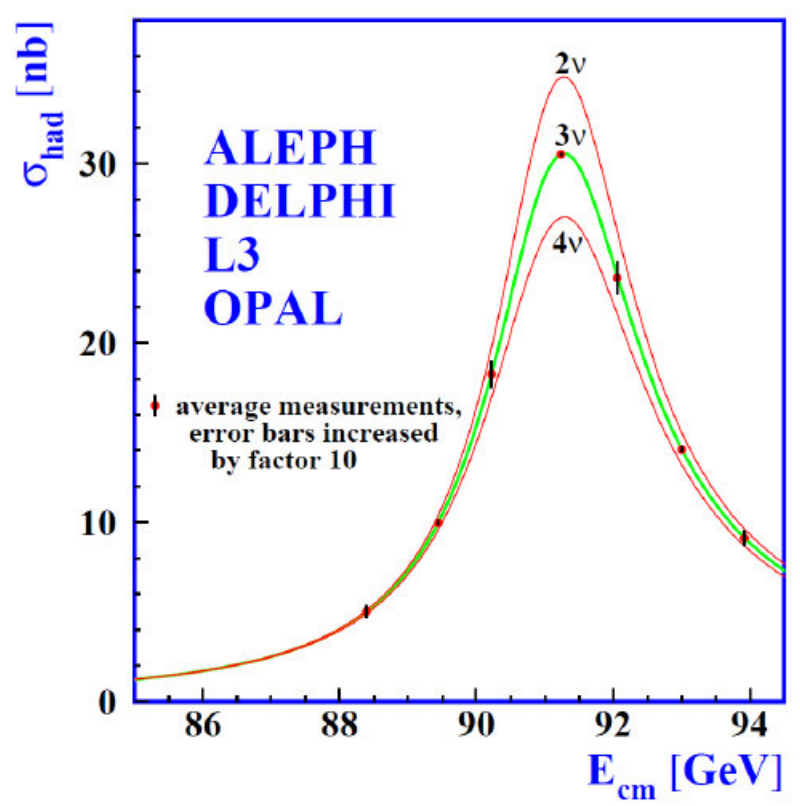

Figure 1.2: Measurement of the hadron production cross section around the $Z^{0}$ mass resonance at LEP. The curves show the prediction for this cross section with 2,3 , and 4 light active neutrino species. The results constrain $\mathrm{N}_{\nu}$ to $2.984 \pm 0.008$ [39].

as a kink in the track. A total of 9 candidate events were observed in their final dataset, giving conclusive proof for the existence of tau neutrinos [41].

\subsection{Neutrinos in the Standard Model}

The SM of particle physics is a locally gauge invariant theory of massless fields. It uses the gauge group $\mathrm{SU}(3) \times \mathrm{SU}(2) \times \mathrm{U}(1)$, which combines the colour gauge group $\mathrm{SU}(3)$ of the strong interaction with the $\mathrm{SU}(2) \times \mathrm{U}(1)$ gauge group of Glashow, Weinberg, and Salam's electroweak theory [28, 29, 30] (GWS). The SM describes the interactions of 17 fundamental particles: the 12 spin- $\frac{1}{2}$ fermions and the 5 spin- 0 and spin-1 bosons [42]. The 17 particles of the SM are shown in Table. 1.1. The fermions are of two kinds: leptons and quarks. Leptons are the electron, muon and tau particles, along with a neutrino associated with each family. Leptons are found free in nature while quarks occur in a bound state that make up hadrons; three-quark states form baryons and quark-antiquark pairs form mesons. They interact via exchange of vector bosons mediating three forces: the strong, electromagnetic 


\begin{tabular}{|c|c|c|c|c|}
\hline \multicolumn{5}{|c|}{ Fermions } \\
\hline & Name & Charge & Spin & Mass (MeV) \\
\hline \multirow{6}{*}{ 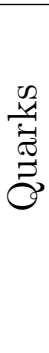 } & $u$ & $+2 / 3$ & $1 / 2$ & $2.3_{-0.5}^{+0.7}$ \\
\hline & $c$ & $+2 / 3$ & $1 / 2$ & $(1.275 \pm 0.025) \times 10^{3}$ \\
\hline & $t$ & $+2 / 3$ & $1 / 2$ & $(173.07 \pm 0.52 \pm 0.72) \times 10^{3}$ \\
\hline & $d$ & $-1 / 3$ & $1 / 2$ & $4.8_{-0.3}^{+0.5}$ \\
\hline & $s$ & $-1 / 3$ & $1 / 2$ & $95 \pm 5$ \\
\hline & $b$ & $-1 / 3$ & $1 / 2$ & $(4.18 \pm 0.03) \times 10^{3}$ \\
\hline \multirow{6}{*}{ 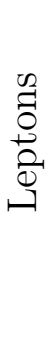 } & $e$ & -1 & $1 / 2$ & $0.51 \pm 1.3 \times 10^{-8}$ \\
\hline & $\mu$ & -1 & $1 / 2$ & $105.65 \pm 3.5 \times 10^{-6}$ \\
\hline & $\tau$ & -1 & $1 / 2$ & $1776.82 \pm 0.16$ \\
\hline & $\nu_{e}$ & 0 & $1 / 2$ & $<2 \times 10^{-6}$ \\
\hline & $\nu_{\mu}$ & 0 & $1 / 2$ & $<2 \times 10^{-6}$ \\
\hline & $\nu_{\tau}$ & 0 & $1 / 2$ & $<2 \times 10^{-6}$ \\
\hline
\end{tabular}

\begin{tabular}{|c|c|c|c|}
\hline \multicolumn{4}{|c|}{ Bosons } \\
\hline Name & Charge & Spin & Mass $(\mathrm{GeV})$ \\
\hline$\gamma$ & 0 & 1 & 0 \\
$W^{ \pm}$ & \pm 1 & 1 & $80.385 \pm 0.015$ \\
$Z^{0}$ & 0 & 1 & $91.188 \pm 0.002$ \\
$g$ & 0 & 1 & 0 \\
$H^{0}$ & 0 & 0 & $125 \pm 0.4$ \\
\hline
\end{tabular}

Table 1.1: Particles of the SM. The particle masses are as currently measured and are obtained from [42, 43, 44].

and weak interactions. The strong interactions are responsible for the interaction of the nucleons and lead to nuclear binding. There are eight vector bosons called gluons which mediate the strong interaction. The weak interaction is mediated by two charged $\left(\mathrm{W}^{ \pm}\right)$ and one neutral $\left(\mathrm{Z}^{0}\right)$ massive vector boson. It is responsible for radioactive decays. The electromagnetic interaction couples to all charged quarks and leptons, and is mediated by the photon. Particle masses in the SM are introduced by the Higgs mechanism of spontaneous symmetry breaking [45]. Neutrinos are massless in the SM. 


\subsection{Neutrino Masses and Mixing}

In 1958 Bruno Pontecorvo first came up with the idea of neutrino masses, mixing and oscillations [46, 47], similar to that seen in the neutral kaon system [48]. At that time only one type of neutrino was known and his model suggested oscillations between neutrinos and antineutrinos, assuming non-conservation of lepton number in the neutrino sector. In 1967, following the discovery of $\nu_{\mu}$, he considered various models involving $\nu_{e} \rightarrow \nu_{\mu}$ oscillations. The formalism of neutrino mixing as we know it today was given by Ziro Maki, Masaki Nakagawa and Shoichi Sakata in 1962 [49], discussed below:

The formulism of neutrino oscillations given by Pontecorvo, Maki, Nakagawa, and Sakata (PMNS) was for two flavour of neutrinos. Here, it is discussed for a general case of any number of neutrinos. The three-flavour and two-flavour scenarios are discussed later as special cases of it. The notation used is from [50].

The neutrino flavour eigenstates $\left|\nu_{\alpha}\right\rangle$ are mixtures of the mass eigenstates $\left|\nu_{i}\right\rangle$. The flavour states are the states that couple to $W^{ \pm}$and $Z^{0}$ bosons. Therefore, the weak interaction for neutrinos occurs via flavour eigenstates and they propagate in vacuum in mass eigenstates. A neutrino created at time $t=0$ in weak flavour eigenstate $\left|\nu_{\alpha}(0)\right\rangle$ can be written as a sum of mass eigenstates:

$$
\left|\nu_{\alpha}(0)\right\rangle=\sum_{i} U_{\alpha i}^{*}\left|\nu_{i}\right\rangle
$$

According to the Schrödinger equation the time evolution of the neutrino state is given by

$$
\left|\nu_{\alpha}(t)\right\rangle=e^{-i H t}\left|\nu_{\alpha}(0)\right\rangle
$$

where $H$ is the Hamiltonian and $\left|\nu_{\alpha}(0)\right\rangle=\left|\nu_{\alpha}\right\rangle$ is the state of the neutrino at time $t=0$. Here $\left|\nu_{\alpha}\right\rangle$ is not an eigenstate of the Hamiltonian, but the mass basis states are eigenstates of the Hamiltonian,

$$
H\left|\nu_{i}\right\rangle=E_{i}\left|\nu_{i}\right\rangle
$$


with eigenvalues $E_{i}=\sqrt{p^{2}+m_{i}^{2}}$. Therefore, using this relation and the equation 1.8 the time evolution of the neutrino state can be written as:

$$
\left|\nu_{\alpha}(t)\right\rangle=\sum_{i=1}^{N} e^{-i E_{i} t} U_{\alpha i}^{*}\left|\nu_{i}\right\rangle
$$

where $N$ is the number of neutrino mass states. Since the neutrino state interact only via flavour eigenstates, we write $\left|\nu_{i}\right\rangle$ in terms of $\left|\nu_{\alpha}\right\rangle$ by inverting equation 1.8 (using the fact that $\mathrm{U}$ is a unitary matrix):

$$
\left|\nu_{\alpha}(t)\right\rangle=\sum_{\beta} \sum_{i=1}^{N} e^{-i E_{i} t} U_{\beta i} U_{\alpha i}^{*}\left|\nu_{\beta}\right\rangle
$$

where $\beta$ sums over the flavour states.

The probability of observing a neutrino flavour state, $\left|\nu_{\beta}\right\rangle$, starting from state $\left|\nu_{\alpha}\right\rangle$ after time $t$ is given by squaring the amplitude:

$$
\begin{aligned}
P_{t}\left(\nu_{\alpha} \rightarrow \nu_{\beta}\right) & =\left|\left\langle\nu_{\beta} \mid \nu_{\alpha}(t)\right\rangle\right|^{2} \\
& =\left|\sum_{i=1}^{N} e^{-i E_{i} t} U_{\beta i} U_{\alpha i}^{*}\right|^{2} \\
& =\sum_{i=1}^{N} \sum_{j=1}^{N} U_{\alpha i}^{*} U_{\alpha j} U_{\beta i} U_{\beta j}^{*} e^{-i\left(E_{i}-E_{j}\right) t}
\end{aligned}
$$

Because of their very small mass, neutrinos are highly relativistic and one can make the approximation: $E_{i} \approx p+\frac{m_{i}^{2}}{2 p}$. Natural units (where $c=1$ ) are used to convert the time of travel to distance, $L$. Since all neutrinos are produced coherently $p$ is the same for all mass states $i$. The probability is then given by:

$$
P_{L}\left(\nu_{\alpha} \rightarrow \nu_{\beta}\right)=\sum_{i=1}^{N} \sum_{j=1}^{N} U_{\alpha i}^{*} U_{\alpha j} U_{\beta i} U_{\beta j}^{*} e^{-i \frac{L}{2 E} \Delta m_{i j}^{2}}
$$

where $\Delta m_{i j}^{2}=m_{i}^{2}-m_{j}^{2}$ and the approximation $p=E$ is made because of the small mass of 
neutrinos. The expression for probability can be more conveniently written as:

$$
\begin{aligned}
P_{L}\left(\nu_{\alpha} \rightarrow \nu_{\beta}\right)=\delta_{\alpha \beta} & -4 \sum_{i>j} \operatorname{Re}\left(U_{\beta i} U_{\beta j}^{*} U_{\alpha i}^{*} U_{\alpha j}\right) \sin ^{2}\left(\frac{\Delta m_{i j}^{2} L}{4 E}\right) \\
& +2 \sum_{i>j} \operatorname{Im}\left(U_{\beta i} U_{\beta j}^{*} U_{\alpha i}^{*} U_{\alpha j}\right) \sin \left(\frac{\Delta m_{i j}^{2} L}{2 E}\right) .
\end{aligned}
$$

where Re and Im denote the real and imaginary parts of the equation. Equation 1.15 gives the probability that a neutrino generated in flavour eigenstate $\alpha$ will be observed in the flavour eigenstate $\beta$ at a distance $L$ from the source, for any number of neutrino states. It is obvious that oscillations are possible only if neutrinos are massive, and only if at least two of the mass eigenstates $\nu_{i}$ have different masses.

Let us examine some symmetry properties of this expression. We see that time reversal, where $\alpha$ and $\beta$ are swapped, is equivalent to swapping $U$ and $U^{*}$,

$$
P_{L}\left(\nu_{\alpha} \rightarrow \nu_{\beta} ; U\right)=P_{L}\left(\nu_{\beta} \rightarrow \nu_{\alpha} ; U^{*}\right)
$$

and $\mathcal{C P} \mathcal{T}$-invariance requires that

$$
P_{L}\left(\bar{\nu}_{\alpha} \rightarrow \bar{\nu}_{\beta}\right)=P_{L}\left(\nu_{\beta} \rightarrow \nu_{\alpha}\right)
$$

Combining these two requirements gives:

$$
P_{L}\left(\nu_{\alpha} \rightarrow \nu_{\beta} ; U\right)=P_{L}\left(\bar{\nu}_{\alpha} \rightarrow \bar{\nu}_{\beta} ; U^{*}\right)
$$

Therefore if $U$ is real, in which case $U=U^{*}$, the transition probability is $\mathcal{C P}$-invariant and $P_{L}\left(\nu_{\alpha} \rightarrow \nu_{\beta}\right)=P_{L}\left(\bar{\nu}_{\alpha} \rightarrow \bar{\nu}_{\beta}\right)$. Conversely, if it is observed in experiments that $P_{L}\left(\nu_{\alpha} \rightarrow\right.$ $\left.\nu_{\beta}\right) \neq P_{L}\left(\bar{\nu}_{\alpha} \rightarrow \bar{\nu}_{\beta}\right)$, then that would be the evidence of $\mathcal{C P}$-violation. 


\subsubsection{Three Flavour Case}

The previous discussion of neutrino oscillations is for any number of neutrinos. Since the SM predicts three neutrinos corresponding to the three families of quarks and charged leptons, we consider neutrino mixing for the case of three neutrino flavours. Three flavour mixing can be expressed as:

$$
\left(\begin{array}{c}
\nu_{e} \\
\nu_{\mu} \\
\nu_{\tau}
\end{array}\right)=U\left(\begin{array}{l}
\nu_{1} \\
\nu_{2} \\
\nu_{3}
\end{array}\right)
$$

with the PMNS mixing matrix U:

$$
\begin{aligned}
U & =U^{(12)} U^{(13)} U^{(23)} \\
& =\left(\begin{array}{ccc}
c_{12} & s_{12} & 0 \\
-s_{12} & c_{12} & 0 \\
0 & 0 & 1
\end{array}\right)\left(\begin{array}{ccc}
c_{13} & 0 & s_{13} e^{-i \delta} \\
0 & 1 & 0 \\
-s_{13} e^{i \delta} & 0 & c_{13}
\end{array}\right)\left(\begin{array}{ccc}
1 & 0 & 0 \\
0 & c_{23} & s_{23} \\
0 & -s_{23} & c_{23}
\end{array}\right) \\
& =\left(\begin{array}{ccc}
c_{13} c_{12} \\
-c_{23} s_{12}-s_{23} c_{12} s_{13} e^{i \delta} & c_{23} c_{12}-s_{23} s_{12} s_{13} e^{i \delta} & c_{13} s_{23} \\
s_{23} s_{12}-c_{23} c_{12} s_{13} e^{i \delta} & -s_{23} c_{12}-c_{23} s_{12} s_{13} e^{i \delta} & c_{13} s_{23}
\end{array}\right) .
\end{aligned}
$$

\subsubsection{Two Neutrino Mixing}

In order to simplify the mixing matrix Equation 1.22 , the mixing can be approximated as between only two neutrino species. This approximation is reasonable because of the large mass splitting between two of the neutrino states. In the case of MINOS experiment a beam of muon neutrinos of energy $\approx 3 \mathrm{GeV}$ is produced. The beam is allowed to travel a distance of $735 \mathrm{~km}$ before it is made to interact in the detector to measure the number of neutrinos 
remaining. Let us consider transitions between $\nu_{\mu}$ and $\nu_{\tau}$ which are mixtures of neutrino mass state $\nu_{2}$ and $\nu_{3}$. With only two neutrinos, the mixing can be parameterised by one angle. Thus, in Equation 1.15, there will be only one term in the sum $i>j(i=3$ and $j=2$ ) and since the matrix $U$ is real, $U=U^{*}$ and the imaginary term vanishes, leaving,

$$
P_{L}\left(\nu_{\mu} \rightarrow \nu_{\tau}\right)=\delta_{\mu \tau}-4 U_{\mu 3} U_{\mu 2} U_{\tau 3} U_{\tau 2} \sin ^{2}\left(\frac{\Delta m_{32}^{2} L}{4 E}\right)
$$

Substituting in the two-flavour mixing matrix,

$$
U=\left(\begin{array}{cc}
\cos \theta_{23} & \sin \theta_{23} \\
-\sin \theta_{23} & \cos \theta_{23}
\end{array}\right),
$$

the probability becomes,

$$
\begin{aligned}
& P_{L}\left(\nu_{\mu} \rightarrow \nu_{\mu}\right)=1-\sin ^{2} 2 \theta_{23} \sin ^{2}\left(\frac{\Delta m_{32}^{2} L}{4 E}\right) \\
& P_{L}\left(\nu_{\mu} \rightarrow \nu_{\tau}\right)=\sin ^{2} 2 \theta_{23} \sin ^{2}\left(\frac{\Delta m_{32}^{2} L}{4 E}\right)
\end{aligned}
$$

Note that $P_{L}\left(\nu_{\mu} \rightarrow \nu_{\mu}\right)+P_{L}\left(\nu_{\mu} \rightarrow \nu_{\tau}\right)=1$, consistent with the conservation of probability.

Since $U=U^{*}$ in the two-neutrino scenario, the time-reversal (Equation 1.16) and $\mathcal{C P}$ inversion (Equation 1.18) expressions require:

$$
P_{L}\left(\nu_{\mu} \rightarrow \nu_{\tau}\right)=P_{L}\left(\bar{\nu}_{\mu} \rightarrow \bar{\nu}_{\tau}\right)=P_{L}\left(\nu_{\tau} \rightarrow \nu_{\mu}\right)=P_{L}\left(\bar{\nu}_{\tau} \rightarrow \bar{\nu}_{\mu}\right)
$$

Since these symmetry expressions assume only $\mathcal{C P} \mathcal{T}$ conservation, any violation of them is evidence that either $\mathcal{C P} \mathcal{T}$ is not conserved or that the oscillation probability needs to be different for neutrinos and antineutrinos. The analysis described in Chapter 5 is based on the two-flavour approximation derived above. A number of models have also tested $\mathcal{C P} \mathcal{T}$ invariance. One such model will be discussed in the next chapter and will be used for the 
analysis described in Chapter 6 .

\subsubsection{Matter Effects}

The expressions for neutrino oscillations derived above are true only when the neutrinos are travelling through vacuum. When travelling through matter it is necessary to take into account the effect of coherent forward scattering. This effect was first observed by Wolfenstein in 1978 [51]. This model was later applied to the solar neutrino oscillation problem by Mikheyev and Smirnov [52]. This effect is different for electron neutrinos because matter is composed of electrons. While all neutrino flavours experience the neutral current interaction, only $\nu_{e}$ can interact with electrons in the medium via charged-current interactions.

The charged-current scattering in the presence of matter is discussed in the case of twoneutrino oscillations. In the absence of matter the Schödinger equation for the time evolution of the neutrino state in the mass basis is

$$
i \frac{d}{d t}\left(\begin{array}{c}
\nu_{1} \\
\nu_{2}
\end{array}\right)=H_{\mathrm{vac}}\left(\begin{array}{c}
\nu_{1} \\
\nu_{2}
\end{array}\right)
$$

where the Hamiltonian for mass eigenstates is easily determined

$$
H_{\mathrm{vac}}=\left(\begin{array}{cc}
E_{1} & 0 \\
0 & E_{2}
\end{array}\right) \approx p+\left(\begin{array}{cc}
m_{1}^{2} / 2 E & 0 \\
0 & m_{2}^{2} / 2 E
\end{array}\right)
$$

where $p$ is the neutrino momentum, $E$ is the neutrino energy, and $m_{1}, m_{2}$ are the masses of $\nu_{1}, \nu_{2}$. This expression can be multiplied by the matrix $U$ to transform into the weak basis,

$$
\left(\begin{array}{l}
\nu_{e} \\
\nu_{\beta}
\end{array}\right)=U\left(\begin{array}{l}
\nu_{1} \\
\nu_{2}
\end{array}\right)
$$

where one of the weak states has been assumed to be the electron neutrino. Multiplying by 
$U^{\dagger}$ from the left and rearranging, Equation 1.28 becomes

$$
i \frac{d}{d t}\left(\begin{array}{c}
\nu_{e} \\
\nu_{\beta}
\end{array}\right)=U H_{\mathrm{vac}} U^{\dagger}\left(\begin{array}{c}
\nu_{e} \\
\nu_{\beta}
\end{array}\right)
$$

The charged-current scattering of electron neutrinos from electrons in matter adds a matter potential

$$
V_{m}^{e}= \pm \sqrt{2} G_{F} n_{e}
$$

where $G_{F}$ is the Fermi constant, $n_{e}$ is the density of electrons in the medium. This term is negative for antineutrinos. Since only electron neutrinos are affected by the matter interaction, the change appears only in the $e$-e term of the Hamiltonian. The effective Hamiltonian then becomes

$$
H=U H_{\mathrm{vac}} U^{\dagger}+H_{\mathrm{CC}}=\frac{\Delta m^{2}}{2 E}\left(\begin{array}{cc}
\sin ^{2} \theta+\frac{2 E}{\Delta m^{2}} V_{m}^{e} & -\sin \theta \cos \theta \\
-\sin \theta \cos \theta & \cos ^{2} \theta
\end{array}\right)
$$

where terms proportional to the identity matrix have been removed. The modified Hamiltonian is then diagonalised to get

$$
\begin{aligned}
f_{m} & =\sqrt{\sin ^{2} 2 \theta+\left(\cos 2 \theta-\frac{2 E}{\Delta m^{2}} V_{m}^{e}\right)^{2}} \\
E_{1,2}^{m} & = \pm \frac{\Delta m^{2}}{4 E} f_{m} \\
\cos 2 \theta^{m} & =\frac{1}{f_{m}}\left(\cos 2 \theta-\frac{\Delta m^{2}}{2 E} V_{m}^{e}\right) \\
\sin 2 \theta^{m} & =\frac{1}{f_{m}} \sin 2 \theta,
\end{aligned}
$$

where $\theta^{m}$ is the new mixing angle and $E_{1,2}^{m}$ are energy eigenvalues. The final oscillation 
formula then becomes

$$
P\left(\nu_{e} \rightarrow \nu_{e}\right)=1-\frac{\sin ^{2} 2 \theta}{f_{m}^{2}} \sin ^{2}\left(\frac{\Delta m^{2} f_{m} L}{4 E}\right)
$$

The additional $f_{m}$ factors account for the matter effects. The term $\Delta m^{2} f_{m}$ has the form $\left(\Delta m^{2} \cos 2 \theta-2 E V_{m}^{e}\right)^{2}$. Thus if matter effects can be observed it is possible to measure the sign of $\Delta m^{2}$, and hence resolve the mass heirarchy, since the matter effects add or subtract from $\Delta m^{2}$ directly and not from its absolute value.

\subsection{Current Status of Measurements}

Neutrino oscillation experiments can measure the following parameters: $\theta_{12}, \Delta m_{21}^{2}, \theta_{23}$, $\left|\Delta m_{32}^{2}\right|, \theta_{13}$, and a phase $\delta$. The first five parameters have been measured with high precision, and the sixth is yet to be constrained.

\subsubsection{Solar Neutrino Oscillations}

Neutrino disappearance was first observed by Ray Davis in 1968 at the Homestake experiment [53]. The experiment was designed to measure the solar neutrino flux in ${ }^{8} \mathrm{~B}$ decay.

$$
{ }^{8} \mathrm{~B} \rightarrow{ }^{8} \mathrm{Be}^{*}+e^{+}+\nu_{e}(\approx 10 \mathrm{MeV}),
$$

The detector consisted of a tank of $\mathrm{C}_{2} \mathrm{Cl}_{4}$ in which Argon atoms were produced due to the reaction $\nu_{e}+{ }^{37} \mathrm{Cl} \rightarrow e^{-}+{ }^{37} \mathrm{Ar}$. The flux of ${ }^{37} \mathrm{Ar}$ was measured to be less than half of that predicted by the Standard Solar Model (SSM) [54]. At first the cause of this discrepancy was thought to be some problem with the SSM. So the Kamiokande experiment performed the measurement independently with a Cherenkov detector [55] and verified that the discrepancy is observed for atmospheric as well as solar neutrinos. These two experiments were sensitive only to the highest energy solar neutrinos coming from the decay of ${ }^{8} \mathrm{~B}$, but these neutrinos form only a tiny fraction of the total solar neutrino flux and their rate is model-dependent. 
They observed solar neutrino rates that were $28 \% \pm 5 \%$ and $46 \% \pm 8 \%$, respectively, as compared to that predicted by the SSM shown in Figure 1.3 .

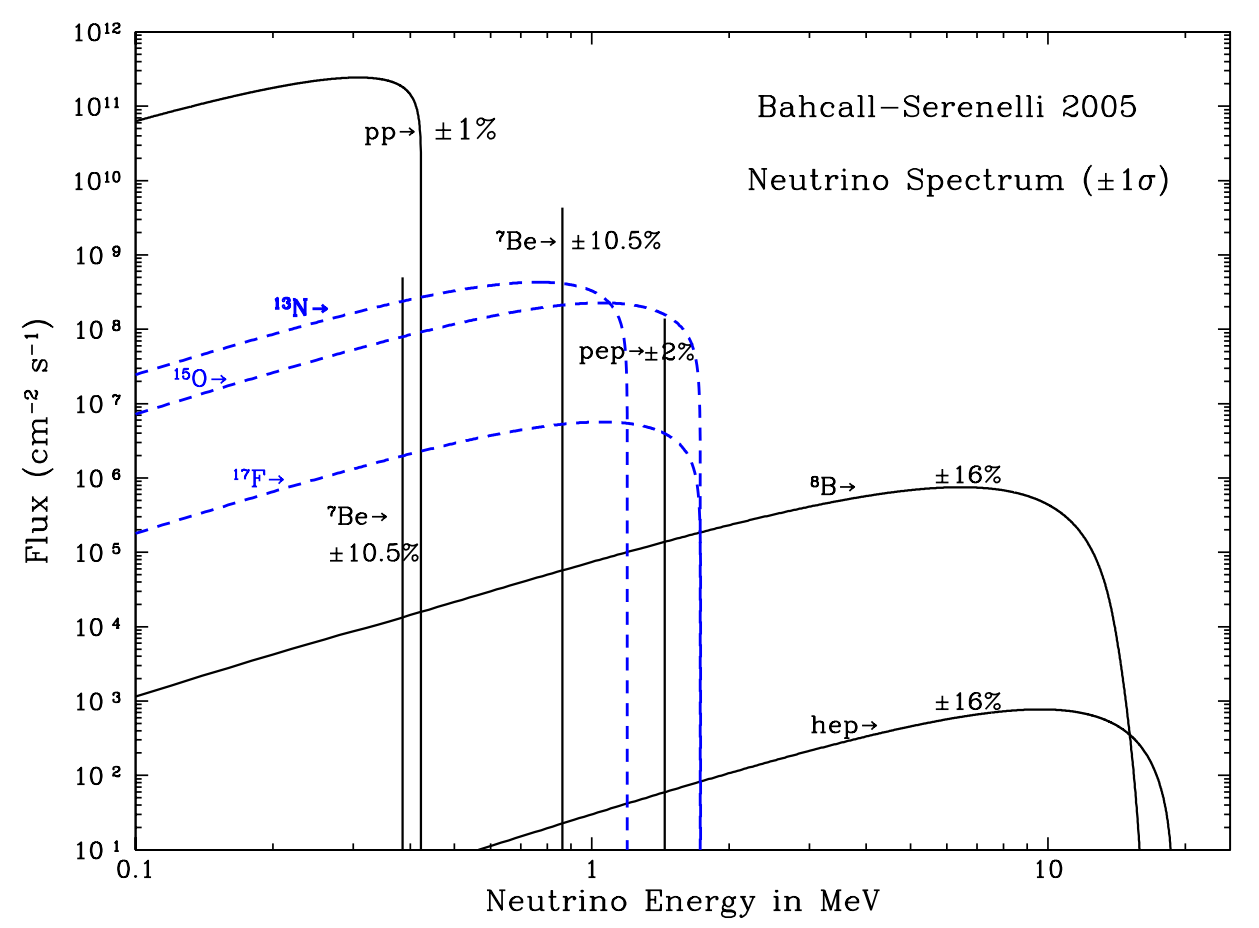

Figure 1.3: The solar neutrino spectrum predicted by the SSM [54]. The numbers associated with the neutrino sources show theoretical errors of the fluxes. (Figure source: http : //www.sns.ias.edu/jnb/.

On the other hand, the $p p$ solar chain, where the Hydrogen nuclei become ${ }^{4}$ He by fusion

$$
p+p \rightarrow d+e^{+}+\nu_{e}(\approx 0.3 \mathrm{MeV})
$$

is understood better. These neutrinos can be observed using the reaction

$$
\nu_{e}+{ }^{71} \mathrm{Ga} \rightarrow e^{-}+{ }^{71} \mathrm{Ge}
$$

The radiochemical experiments, SAGE in Soviet Union [56] and GALLEX-GNO in Italy [57], used this reaction to observe solar neutrinos. This reaction has a very low threshold of $0.223 \mathrm{MeV}$. Therefore, these experiments were sensitive to the $p p$ neutrinos produced in the 
beginning of the main solar fusion reaction. The rate of this recation, unlike that of the ${ }^{8} \mathrm{~B}$ neutrinos, is also mostly model-independent. The rate predicted by the SSM is 128 SNU on Gallium but Sage and Gallex measured rates of $70.8_{-6.1}^{+6.5}$ SNU and $77.5_{-7.8}^{+7.5}$ SNU respectively (SNU denotes a Solar Neutrino Unit which is defined as $10^{36}$ events/atom/second.), which is almost half of that predicted by the model. This came to be known as the "solar neutrino problem".

However, the results from all these experiments could not constrain any one region of $\Delta m_{21}^{2}-\theta_{12}$ parameter space. The isolation of a single pair of oscillation parameters came from the Sudbury Neutrino Observatory (SNO) experiment [58]. The SNO experiment is located about $2 \mathrm{~km}$ underground in Vale's Creighton Mine in Sudbury, Ontario, Canada. It consists of a water Cherenkov detector designed to detect solar neutrinos with $\mathrm{D}_{2} \mathrm{O}$ as the detecting medium. It is capable of measuring the charged and neutral current interaction rates separately and, hence, can test the neutrino oscillation hypothesis in a model-independent way. The results from the experiment isolated the Large Mixing Angle (LMA) solution with MSW matter effects [59]. The combined fit to all solar neutrino data gave the following values of oscillation parameters [60]:

$$
\Delta m_{21}^{2}=\left(5.6_{-1.4}^{+1.9}\right) \times 10^{-5} \mathrm{eV}^{2}, \quad \tan ^{2} \theta_{12}=0.427_{-0.029}^{+0.033}
$$

From the sign of the MSW effect, solar neutrino oscillation measurements established that $m_{2}>m_{1}[42$.

Another way of measuring neutrino oscillations in the 'solar sector' (where the 1-2 oscillations are dominant) is to measure the $\nu_{e}$ from other sources. One such alternative source is nuclear reactors, which provide a flux of $\bar{\nu}_{e}$, and the KamLAND is one of the first experiments to measure reactor antineutrinos [61]. KamLAND is situated in the Kamioka mine in Japan which uses 1 kTon of liquid scintillator surrounded by non-scintillating buffer oil. The light from the scintillator is captured by approximately 1,900 phototubes. The neutrinos 
are detected via inverse beta decay and a prompt-delayed double coincidence, as used in the earliest neutrino experiments, is used as the signal. A total of 55 commercial nuclear reactors, at a distance ranging from 140 to $210 \mathrm{~km}$ from the detector, provide the flux of $\bar{\nu}_{e}$ 's. A significant $\bar{\nu}_{e}$ disappearance [61] was observed by the KamLAND, which, when fit for oscillations, gave

$$
\Delta m_{21}^{2}=\left(7.66_{-0.20}^{+0.22}\right) \times 10^{-5} \mathrm{eV}^{2}, \quad \tan ^{2} \theta_{12}=0.52_{-0.10}^{+0.16}
$$

Fig. 1.4 shows the ratio of the measured to the expected spectra. These results are consistent with the parameters measured by the solar experiments in Equation 1.42. Fig. 1.5 shows the contours from KamLAND and the combined solar experiments. This measurement greatly increased our confidence in neutrino oscillations since it was performed by two independent experiments in a completely different setup.

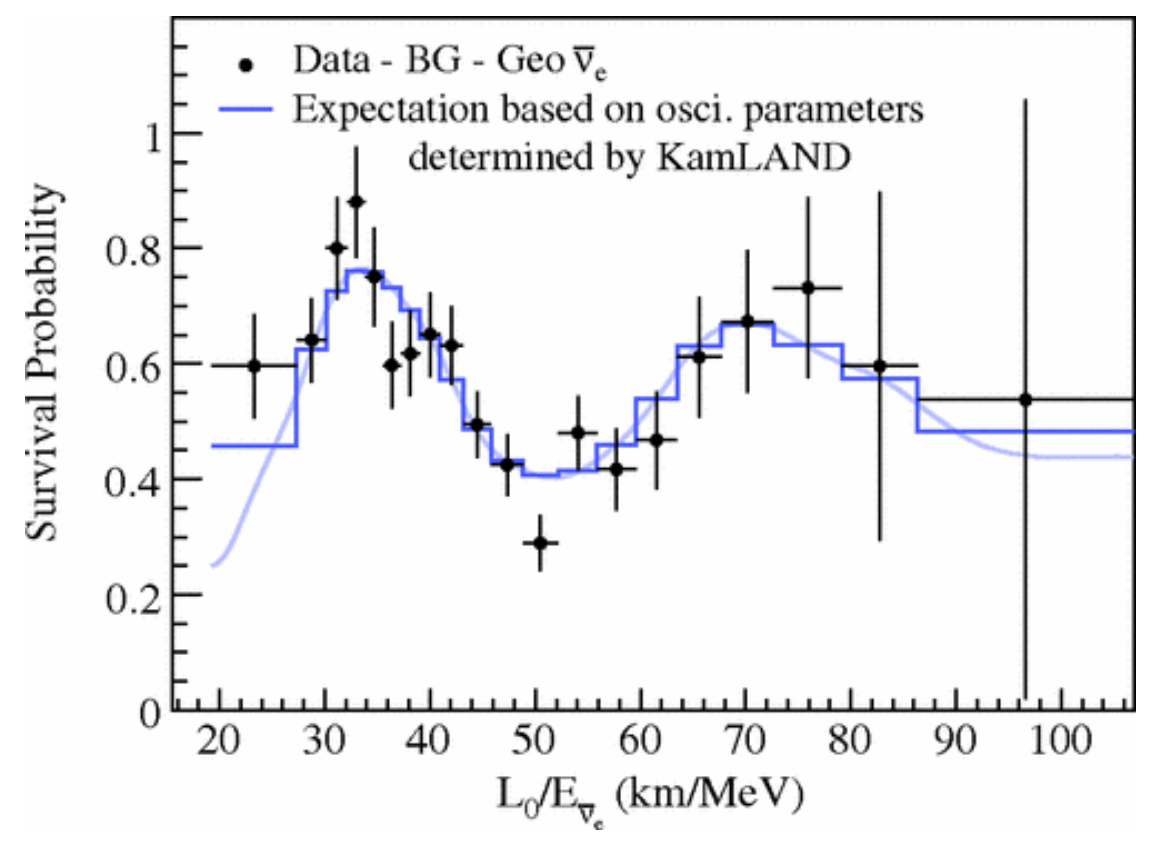

Figure 1.4: Ratio of the measured $\bar{\nu}_{e}$ spectrum to the expectation for no-oscillation as a function of $L_{0} / E$, where $L_{0}$ is the distance travelled and $\mathrm{E}$ is neutrino energy. Also shown is the best fit for oscillation hypothesis. Figure from [61]. 


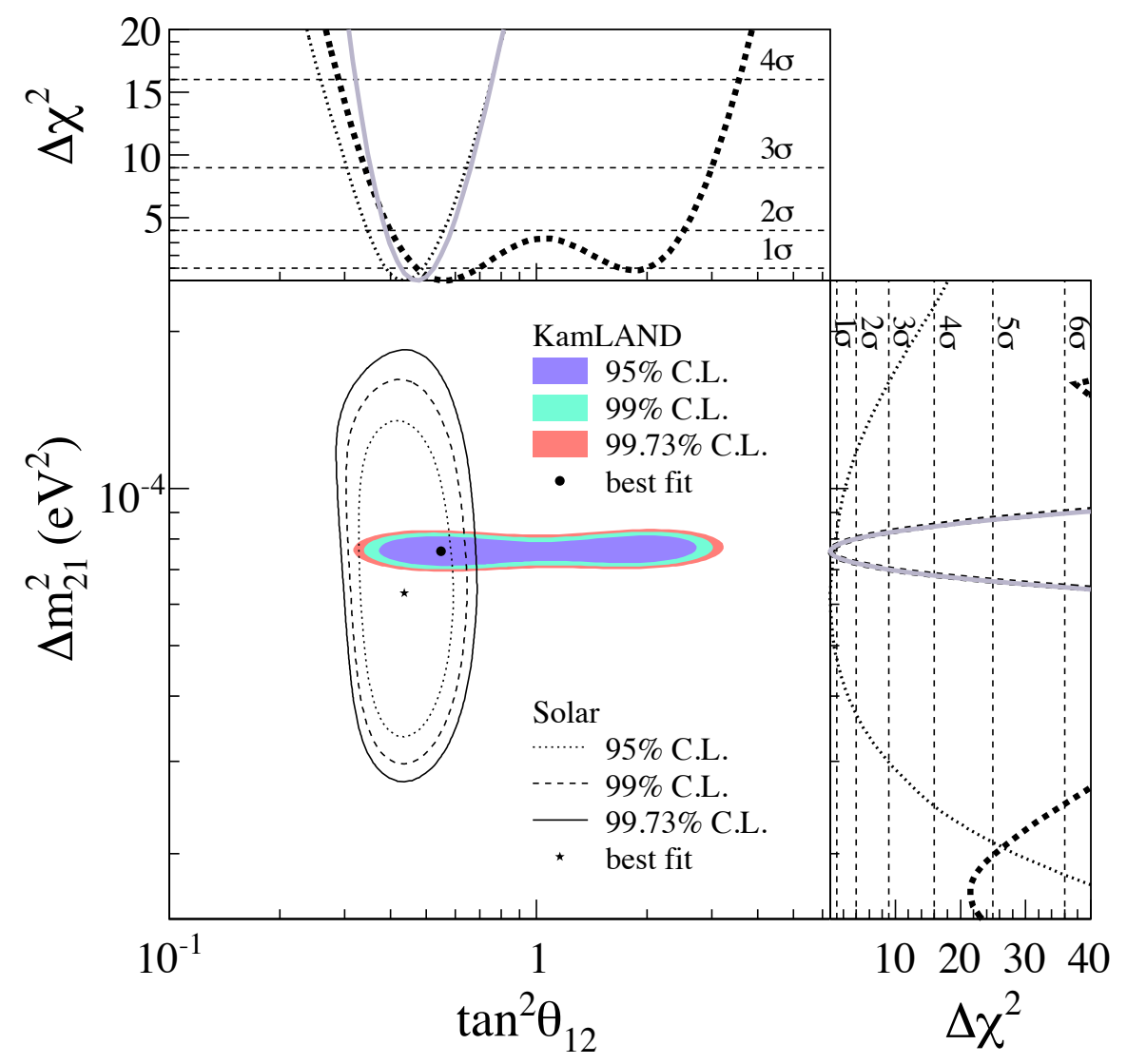

Figure 1.5: The oscillation parameter regions allowed by the KamLAND experiment (solid colours) and the combined solar experiments (black lines). The side plots show the two one-dimensional profiles for KamLAND (dashed), solar (dotted), and combined (solid). The consistency between the solar and reactor measurements lends significant support to the oscillation model of neutrino disappearance. Figure from [61].

The results from the solar and KamLAND experiments are combined, and yields the world's best measurement, with a precision better than $3.5 \%$ [62]:

$$
\Delta m_{21}^{2}=\left(7.54_{-0.22}^{+0.26}\right) \times 10^{-5} \mathrm{eV}^{2}, \quad \sin ^{2} \theta_{12}=0.307_{-0.016}^{+0.018}
$$

\subsubsection{Atmospheric Neutrino Oscillations}

The second set of oscillation parameters, $\Delta m_{23}^{2}$ and $\sin ^{2}\left(2 \theta_{23}\right)$, can be measured using atmospheric neutrinos and accelerator based neutrino beams. When cosmic rays strike the atmosphere they produce pions and kaons. These then decay into neutrinos primarily via: 
$\pi^{ \pm} \rightarrow \mu^{+}+\nu_{\mu} ; \mu^{+} \rightarrow e^{+}+\nu_{e}+\bar{\nu}_{\mu}$. If there were no oscillations one would expect a flux ratio for $\nu_{\mu}: \nu_{e}$ to be $2: 1$. However, the observed $\nu_{\mu}$ flux was $\frac{2}{3}$ the prediction [63]. The best measurement for these parameters comes from the experiments Super-Kamiokande and MINOS.

The first compelling evidence for neutrino oscillations with $\nu_{\mu}$ from accelerator was presented by the Super Kamiokande Collaboration in 1998 [2] from the study of atmospheric neutrinos. The zenith angle distributions of charged-current interactions due to muon neutrinos and muon antineutrinos, shown in Figure 1.6, clearly shows a deficit compared to the distribution if there were no oscillations.
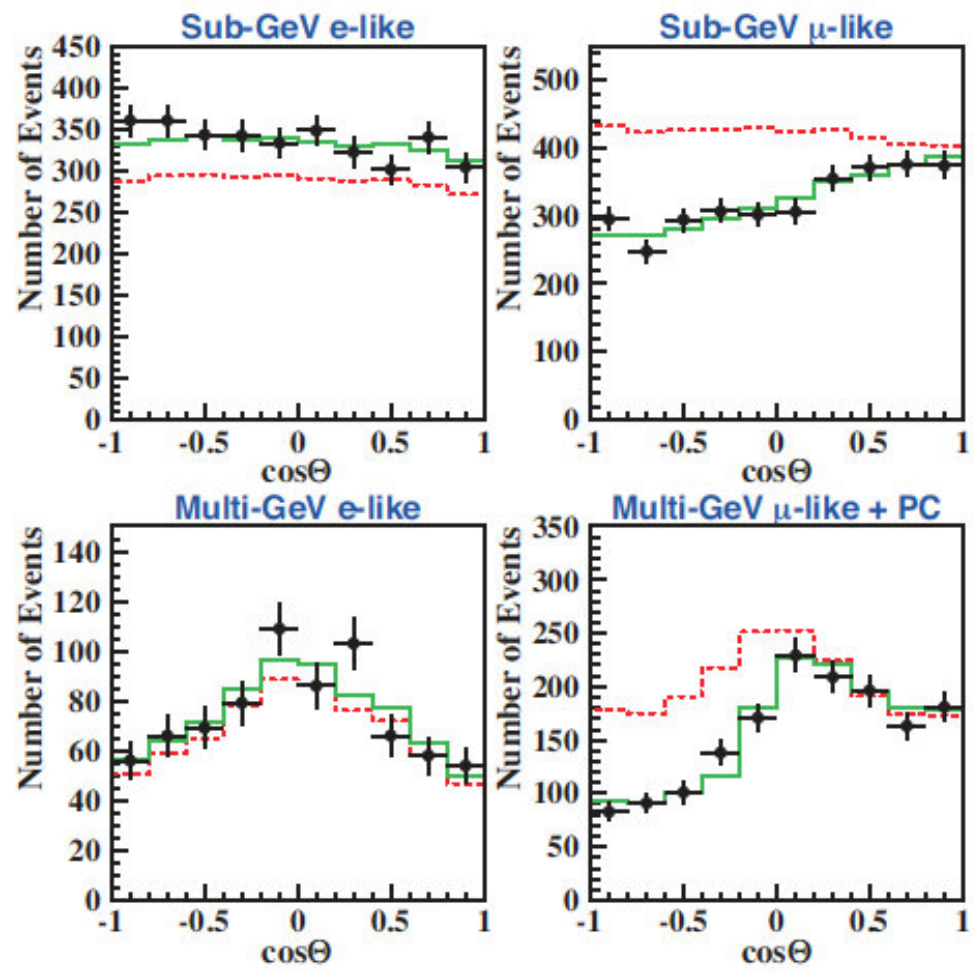

Figure 1.6: Zenith angle distributions for e-like and $\mu$-like events with visible energy < $1.33 \mathrm{GeV}$ (sub-GeV) and $>1.33 \mathrm{GeV}$ (multi-GeV). The dotted histograms show the nonoscillated Monte Carlo events, and the solid histograms show the best-fit expectations for $\nu_{\mu} \rightarrow \nu_{\tau}$ oscillations. Figure from [42].

The atmospheric oscillations were independently confirmed by experiments using muon neutrinos from accelerator beams. The neutrino sources and detectors in the experiments were separated by some distance $L$, and the neutrino beam energy $E$ was tuned such that 
the location of the detector corresponds to one 'oscillation length'. This is the distance over which a neutrino with energy $E$ will oscillate back to its original flavour state. The oscillation length is given by

$$
L_{\mathrm{atm}}=4 \pi \frac{E \hbar c}{\Delta m^{2} c^{4}} \approx 2.5 \frac{E}{\Delta m^{2}} \mathrm{~m}
$$

where $E$ is the neutrino energy in $\mathrm{MeV}$ and $\Delta m^{2}$ is the neutrino mass-splitting in $\mathrm{eV}^{2}$. The first confirmation of neutrino oscillations was obtained from the $\mathrm{K} 2 \mathrm{~K}$ experiment in Japan. Approximately $1 \mathrm{GeV}$ muon neutrinos were produced at the KEK accelerator, and were observed in the Super-Kamiokande detector $250 \mathrm{~km}$ away (For $E=1 \mathrm{GeV}, L_{\text {atm }} \approx 1,000 \mathrm{~km}$ with the $\Delta m^{2}$ measured by Super-Kamiokande). The results were found to be consistent with those obtained from Super-Kamiokande.

The MINOS experiment, which gives the most precise accelerator measurement to date, uses neutrinos with $E \approx 3 \mathrm{GeV}$ and a baseline of $735 \mathrm{~km}$. The experiment is described in detail in the rest of the thesis. By observing the disappearance of muon neutrinos and antineutrinos MINOS has produced the most precise measurement of the atmospheric mass splitting. The best measurement of the mixing angle $\theta_{23}$ is obtained by Super-Kamiokande. The confidence limits on the parameters $\Delta m^{2}$ and $\sin ^{2} 2 \theta$ are shown in Fig. 1.7. The best fit to $\Delta m^{2}$ from MINOS [64] and $\sin ^{2} 2 \theta$ from Super-K [65] are:

$$
\Delta m_{32}^{2}=2.41_{-0.10}^{+0.09} \times 10^{-3} \mathrm{eV}^{2} \quad \sin ^{2}\left(2 \theta_{23}\right)=0.95_{-0.036}^{+0.035}(90 \% \text { C.L. })
$$

\subsubsection{Reactor Neutrino Oscillations}

The mixing angle, $\theta_{13}$, drives the disappearance of electron (anti)neutrinos at the atmospheric oscillation length (Equation 1.45) with the survival probability:

$$
P\left(\bar{\nu}_{e} \rightarrow \bar{\nu}_{e}\right)=1-\sin ^{2} 2 \theta_{13} \sin ^{2}\left(1.27 \Delta m_{\mathrm{atm}}^{2} \frac{L}{E}\right)
$$




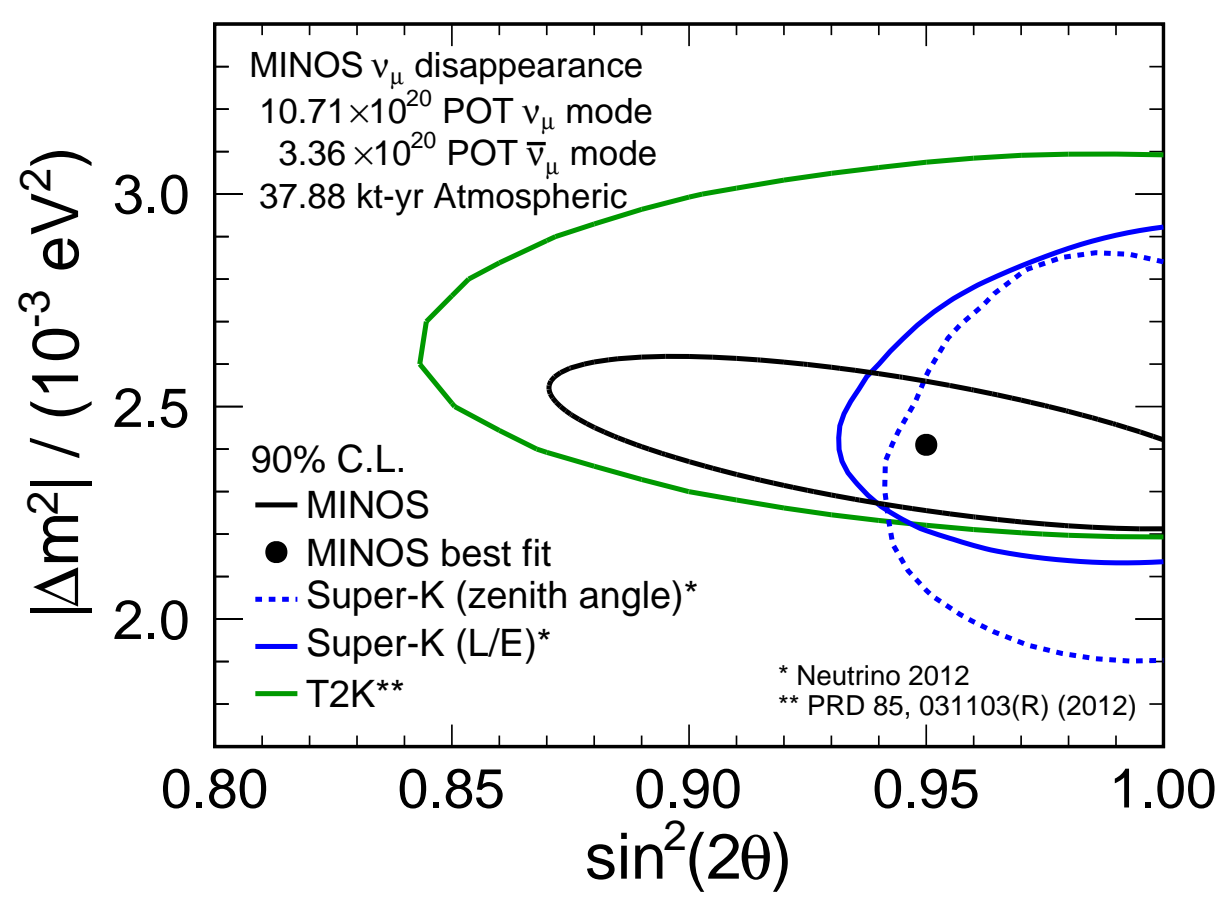

Figure 1.7: Confidence limits on the parameters $\left|\Delta m^{2}\right|$ and $\sin ^{2} 2 \theta$, assuming equal oscillations for neutrinos and antineutrinos. The black curve gives the $90 \%$ contour obtained from the combined analysis of MINOS accelerator and atmospheric neutrinos. For comparison, the blue curves show the $90 \%$ contours from Super-K (zenith angle analysis indicated by dotted line, L/E analysis indicated by solid line) [64]; and the green curve shows the $90 \%$ contour from T2K [66].

where $L$ is in metres and $E$ in $\mathrm{MeV}$. The CHOOZ experiment in France searched for the disappearance of reactor electron antineutrinos $(E \approx 3.6 \mathrm{MeV})$. The electron antineutrinos came from two reactors, with a thermal power of $4.25 \mathrm{GW}$, at the CHOOZ nuclear power plant. The detector, situated $1 \mathrm{~km}$ from the reactors, contained 5 tons of liquid scintillator target doped with Gd (to enhance neutron capture) and the experiment searched for inverse beta-decay. No electron neutrino disappearance was observed, and hence only an upper limit could be set on the value of the last mixing angle. The $\bar{\nu}_{e} \leftrightarrow \bar{\nu}_{\mu}$ oscillations were excluded at $90 \%$ C.L. for $\Delta m^{2}>7 \times 10^{-4} \mathrm{eV}^{2}$ at maximal mixing, and $\sin ^{2} 2 \theta>0.10$ for large $\Delta m^{2}$ [67]. Another experiment, complementary to CHOOZ in detector design was the neutrino experiment at Palo Verde in Arizona. The source of $\bar{\nu}_{e}$ in this case were three 
nuclear reactors: two at a distance of $890 \mathrm{~m}$ from the detector and one at $750 \mathrm{~m}$, with a total thermal output of $11.63 \mathrm{GW}$. The detector consisted of 11.2 tons of Gd-loaded liquid scintillator. Again, no evidence for disappearance of electron antineutrinos was found and the mode $\bar{\nu}_{e} \leftrightarrow \bar{\nu}_{x}$ was excluded at $90 \%$ C.L. for $\Delta m^{2}>1.1 \times 10^{-3} \mathrm{eV}^{2}$ at full mixing, and $\sin ^{2} 2 \theta>0.17$ at large $\Delta m^{2}[68]$.

Another way to measure the angle $\theta_{13}$ is by looking for $\nu_{e}$ appearance in a $\nu_{\mu}$ beam with experiments such as MINOS [69] and T2K [70]. Both these experiments measured $\theta_{13}$ to be greater than zero and global fits to all available data gave $>3 \sigma$ evidence for nonzero $\theta_{13}$, with the central values in the range $0.05<\sin ^{2} 2 \theta_{13}<0.10$ [71, 72]. The Double Chooz experiment was the successor to the $\mathrm{CHOOZ}$ experiment, consisting of two identical detectors, one at $400 \mathrm{~m}$ and the other at $1.05 \mathrm{~km}$ distance from the CHOOZ nuclear cores. It was designed to be sensitive to the above mentioned range of $\theta_{13}$. The experiment observed 8249 candidate electron antineutrino events with $33.71 \mathrm{GW}$-ton-years exposure using the detector located at $10.5 \mathrm{~km}$. The expectation in the case of $\theta_{13}=0$ was 8937 events. From a rate and spectral shape analysis it was found that $\sin ^{2} 2 \theta_{13}=0.109 \pm 0.030$ (stat) \pm 0.025 (syst) [73].

A very precise measurement of the mixing angle was later made by the reactor experiment Daya Bay in China. The Daya Bay nuclear power complex consists of 6 reactors, each with a maximum of $2.9 \mathrm{GW}$ thermal power $\left(\mathrm{GW}_{t h}\right)$, producing a high flux of $\approx 3 \mathrm{GeV}$ electron antineutrinos. There are two near detectors and one far detector situated at approximately $470 \mathrm{~m}, 576 \mathrm{~m}$ and $1648 \mathrm{~m}$, respectively, from the nuclear reactors. The electron antineutrinos are detected via inverse $\beta$-decay in gadolinium doped liquid scintillator. They looked for the coincidence of the prompt scintillation from the $e^{+}$and the delayed neutrons captured on Gd. The mixing angle $\theta_{13}$ was obtained by comparing the observed and expected rates at the far detector and the difference in the spectral shape. The best fit to their data gave [74]

$$
\sin ^{2} \theta_{13}=0.089_{-0.005}^{+0.010}
$$


Fig. 1.8 shows the measured prompt energy spectrum of the far hall compared with the nooscillation prediction based on the spectrum at the two near halls in Daya Bay experiment.

This measurement was confirmed by the Reactor Experiment for Neutrino Oscillations (RENO) experiment. The RENO experiment is located in Korea and obtains antineutrinos from six $2.8 \mathrm{GW}_{t h}$ reactors at the Yonggwang Nuclear Power Plant. The antineutrinos are detected by two identical detectors located at 294 and $1383 \mathrm{~m}$, respectively, from the reactor array centre. By comparing the observed and expected rates at the far detector the value of $\sin ^{2} 2 \theta_{13}$ was determined to be $0.113 \pm 0.013$ (stat) \pm 0.019 (syst) [75]. The observed and expected spectra at the far detector along with the ratios of the measured spectra and the no-oscillation prediction are shown in Fig. 1.9.

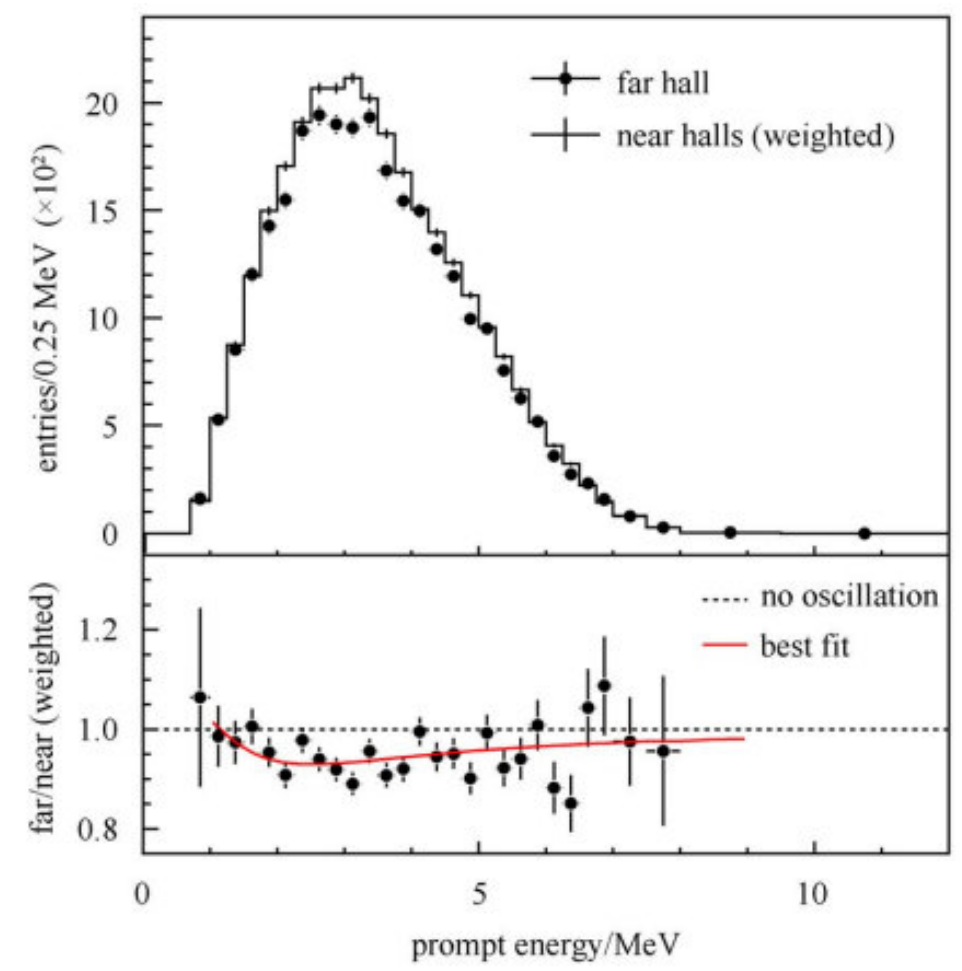

Figure 1.8: Top: Measured prompt energy spectrum of the far hall compared with nooscillation prediction based on the measurements of the two near halls. Bottom: The ratio of measured and predicted no-oscillation spectra. The solid curve shows the expected ratio with oscillations, assuming $\sin ^{2} 2 \theta_{13}=0.089$. The dashed line is the no-oscillation prediction. Figure from [74]. 


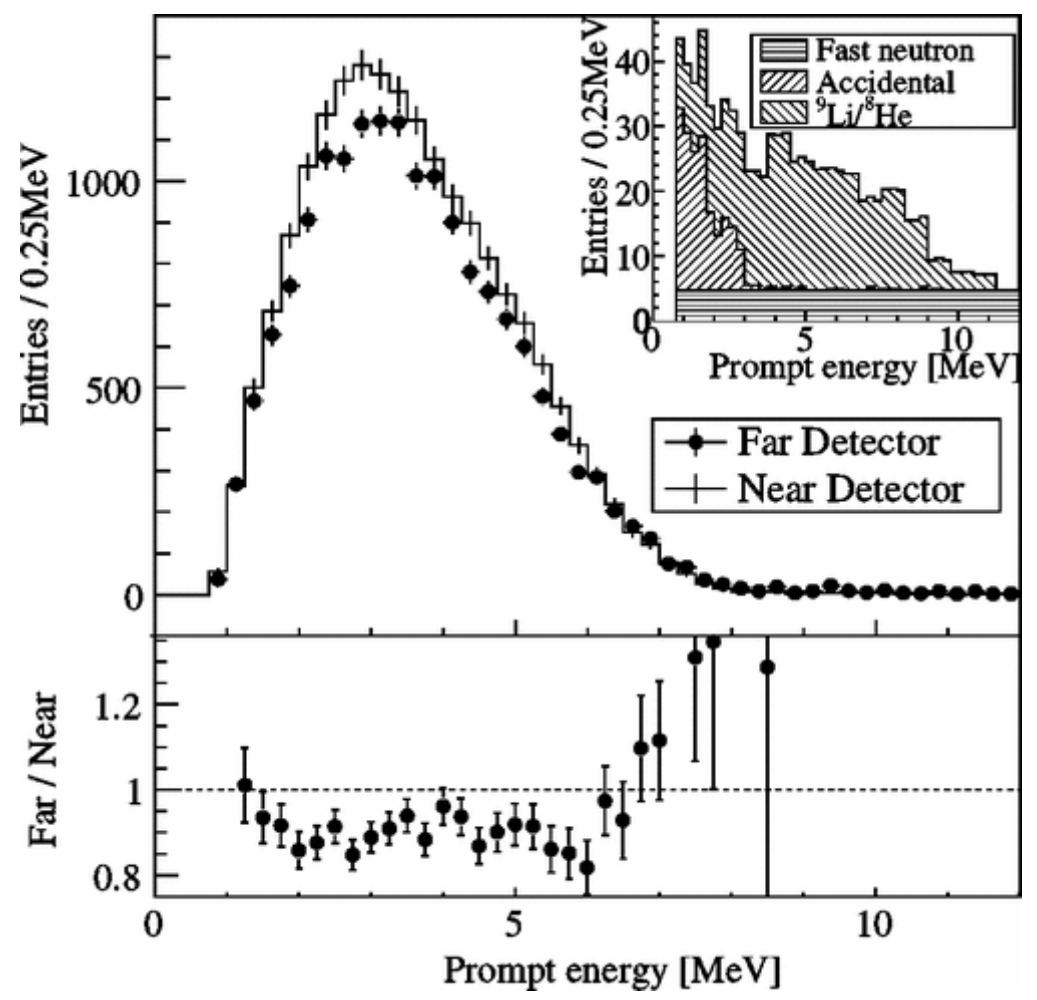

Figure 1.9: Top: Measured prompt energy spectrum of the far detector compared with nooscillation prediction based on the measurements of the near detector. The backgrounds shown in the inset are subtracted from the far detector spectrum. Bottom: The ratio of measured spectrum at the far detector and the no-oscillation prediction. Figure from [75].

The recent updated results from the T2K experiment improve their measurement of the mixing angle. The T2K experiment is a long baseline experiment optimised to observe electron neutrino appearance in a muon neutrino beam. The near detector is at a distance of $280 \mathrm{~m}$ and the far detector at $295 \mathrm{~km}$ from the neutrino production target. A total of 11 $\nu_{e}$ candidate events were observed at the far detector when $3.3 \pm 0.4$ (syst) background events were expected. The background-only hypothesis was rejected with a $p$ value of 0.0009 , which is equivalent to a $3.1 \sigma$ significance. A fit was performed to the far detector events assuming $\delta_{C P}=0$ and $\sin ^{2}\left(2 \theta_{23}\right)=1.0$. The best-fit values of $\sin ^{2}\left(2 \theta_{13}\right)$ with the $68 \%$ confidence levels are [76] 

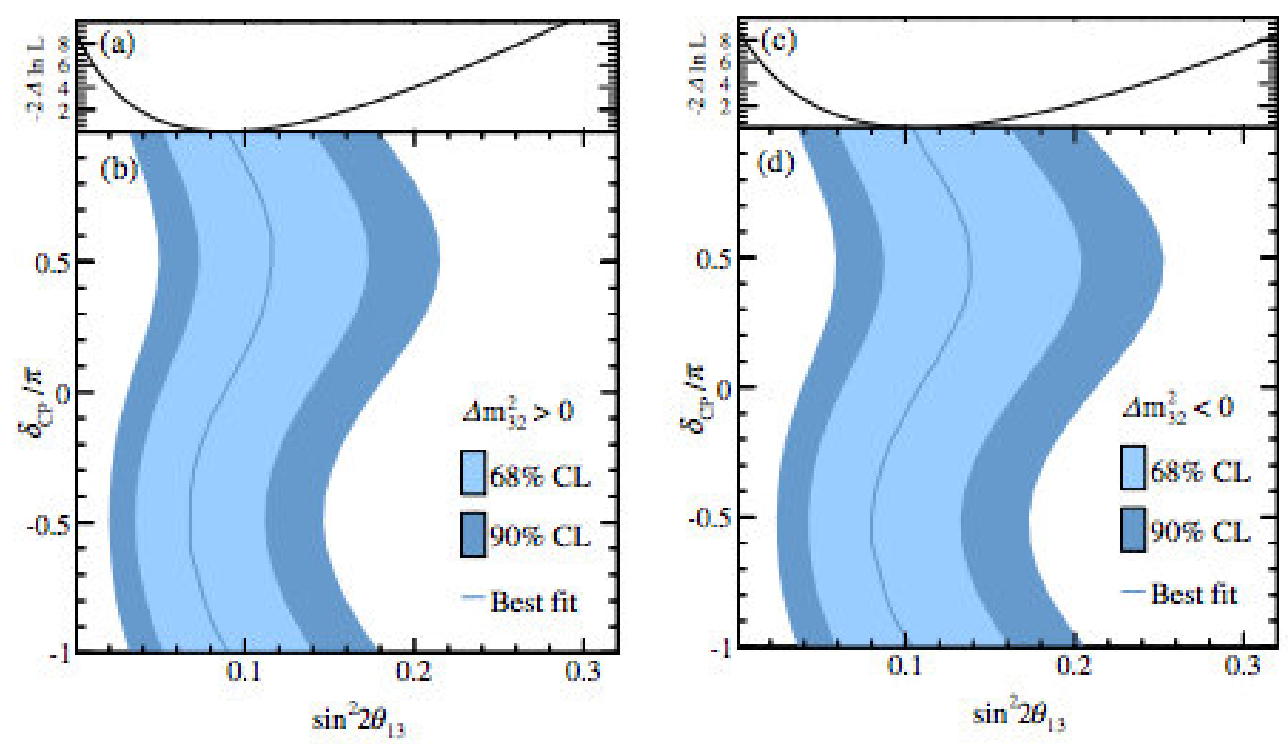

Figure 1.10: The $68 \%$ and $90 \%$ confidence intervals for $\sin ^{2}\left(2 \theta_{13}\right)$ scanned over values of $\delta_{C P}$. The left plot assumes normal hierarchy and the right plot is for inverted hierarchy. Figure from [76].

$$
\begin{aligned}
& \sin ^{2}\left(2 \theta_{13}\right)=0.088_{-0.039}^{+0.049}(\text { normal hierarchy }) \\
& \sin ^{2}\left(2 \theta_{13}\right)=0.108_{-0.046}^{+0.059} \text { (inverted hierarchy). }
\end{aligned}
$$

The allowed confidence intervals are shown in Fig. 1.10.

The above discussion was based on the SM of particle physics and the phenomenon of neutrino oscillations was used to analyse the data from various experiments. It would also be interesting to study whether the existing data can be described by some physical phenomena other than neutrino oscillations. One such model that is formed by adding Lorentz and CPT violating terms to the SM is described in Chapter 2 . 


\section{Chapter 2}

\section{Lorentz and CPT Violation in the}

\section{Neutrino Sector}

\subsection{Standard Model Extension}

The modified SM of particle physics formed by adding neutrino masses to the SM, also called the neutrino SM $(\nu \mathrm{SM})$ [77, has been successful in explaining most of the neutrino oscillation experimental results to date. A large number of experiments have searched for neutrino oscillations and made a precise measurement of oscillation parameters, discussed in Chapter 1. But there is a possibility that there might be new physics beyond the SM. One of the possibilities for new physics is Lorentz violation. The Lorentz symmetry is fundamental to the SM physics and states that the experiments give the same results regardless of their inertial reference frame and regardless of their orientation. A closely related hypothesis is that of CPT symmetry. According to this symmetry the outcome of an experiment is the same if we replace it with its CPT image, by replacing each charge with its conjugate, by reversing the parity of the system, and by reversing time. These two symmetries have been tested by various experiments and no evidence of Lorentz and CPT violation has been found so far. But the anomalous results of some experiments [78, 79, 80, 81] have provided hints of possible symmetry breaking. The discovery of Lorentz or CPT violation would give a 
definite proof of new physics beyond the SM, and would necessitate fundamental changes in the theories of gravity and quantum mechanics. Since violation of these symmetries has never been seen, they are likely to be very small. A challenge of detecting them is to attain sufficient sensitivity to signals of their violation.

The Standard-Model Extension (SME), developed over a period of more than 20 years, is a theoretical framework which provides a quantitative description of Lorentz and CPT violation. It was first published in its basic form in the 1990s [82, 83]. It contains both General Relativity and the SM, and so it is a realistic theory that can be applied to analyse observational and experimental data. It is a realistic effective field theory in which the coefficients for Lorentz violation could arise from spontaneous symmetry breaking in a fundamental theory such as string theory [84, 85]. It exploits the fact that the effects of such symmetry violations can be detected at attainable energies in the context of effective field theory [86, 87]. The SME framework is constructed as a general framework to analyse the data to search for possible Lorentz violation. In the SME, Lorentz violating interactions are described by the perturbative terms in the Lagrangian, on top of the SM terms, controlled by a set of coefficients whose values are to be determined or constrained by experiments. We are particularly interested in the test of Lorentz violation using neutrino oscillations. Since neutrino oscillation is a natural interferometer, small couplings of neutrinos with Lorentz violating fields could cause phase shifts and could result in neutrino oscillations. The sensitivity of neutrino oscillations to Lorentz violation is comparable with precise optical experiments. To analyse the neutrino data the neutrino sector SME [88] has been used in this thesis. Various experiments have investigated their results in the context of SME framework by restricting to a small number of coefficients. The constraints on a large number of SME coefficients have been obtained by different experiments and are compiled in [89]. But the sensitivity to all the coefficients has not been identified yet. The analysis presented in this thesis aims to measure a subset of four time-independent SME coefficients. 


\subsection{The Model used in this Thesis}

The MINOS experiment is suitable for testing Lorentz and CPT violation through evidence of oscillations that deviate from the standard L/E dependence, or that show sidereal time-dependent oscillations as a consequence of a preferred direction in the universe. Previous MINOS analyses have obtained limits on a large number of SME coefficients by looking for sidereal variations [90, 91, 92, 93]. The model used in this analysis considers a different subset of SME coefficients [94] constructed using the basic formalism from [88]. Since MINOS is most sensitive to oscillations in the atmospheric sector, we consider oscillations between neutrino generations $\nu_{\mu}$ and $\nu_{\tau}$ instead of those between $\nu_{e}$ and $\nu_{\mu}$ described in the reference [94]. A two-flavour approximation is considered for simplicity. Because of the presence of Lorentz violation, it is necessary to specify the frame in which experimental results are reported. The measurements are conventionally expressed in terms of coefficients for Lorentz violation defined in a Sun-centered celestial equatorial frame with coordinates ( T, X, Y, Z), where Z lies along the Earth's rotational axis. This makes it easy to compare the results obtained from different experiments. The oscillations depend on the SME coefficients $\left(c_{L}\right)_{\mu \mu}^{T T}\left(\equiv\left(c_{L}\right)_{\mu \mu}^{Z Z}\right),\left(c_{L}\right)_{\tau \tau}^{T T}\left(\equiv\left(c_{L}\right)_{\tau \tau}^{Z Z}\right), \tilde{g}_{\mu \bar{\mu}}^{Z T}$ and $\tilde{g}_{\tau \bar{\tau}}^{Z T}$. Here the $\tilde{g}$-type coefficients are Lorentz as well as CPT violating and the $c$-type coefficients are Lorentz-violating but CPT-conserving. These coefficients have not been constrained by any experiments to date.

It has been shown by Kostelecky and Mewes [88] that a minimal extension of SM physics including all possible CPT and Lorentz symmetry-violating Dirac and Majorana-type coupling to left and right-handed neutrinos gives rise to an effective Hamiltonian $h_{\text {eff }}$ describing neutrino-antineutrino oscillations. In the case of two flavours the Lorentz-violating equations of motion can be written in analogy to the Dirac equation

$$
\left(i \Gamma_{A B}^{\nu} \partial_{\nu}-M_{A B}\right) \nu_{B}=0
$$

where $\mathrm{A}, \mathrm{B}=\mu, \tau, \bar{\mu}$ and $\bar{\tau}$ are flavour indices for neutrinos and antineutrinos, respectively. 
The coefficients $\Gamma_{A B}^{\nu}$ and $M_{A B}$ can be expanded in terms of the $\gamma$ matrices determining the Dirac algebra:

$$
\begin{gathered}
\Gamma_{A B}^{\nu}=\gamma^{\nu} \delta_{A B}+c_{A B}^{\mu \nu} \gamma_{\mu}+d_{A B}^{\mu \nu} \gamma_{5} \gamma_{\mu}+e_{A B}^{\nu}+i f_{A B}^{\nu} \gamma_{5}+\frac{1}{2} g_{A B}^{\lambda \mu \nu} \sigma_{\lambda \mu}, \\
M_{A B}=m_{A B}+i m_{5 A B} \gamma_{5}+a_{A B}^{\mu} \gamma_{\mu}+b_{A B}^{\mu} \gamma_{5} \gamma_{\mu}+\frac{1}{2} H_{A B}^{\mu \nu} \sigma_{\mu \nu}
\end{gathered}
$$

The masses $m$ and $m_{5}$ are CPT and Lorentz-conserving and equivalent to the standard Majorana and Dirac type masses. The coefficients with even number of indices: c, d and $\mathrm{H}$ are CPT-conserving but Lorentz-violating; and those with odd number of indices: a, b, e, f and $\mathrm{g}$ are both CPT and Lorentz-violating. The propagation of the states $\nu_{\mu}, \nu_{\tau}, \bar{\nu}_{\mu}$, and $\bar{\nu}_{\tau}$ is governed by the Schrodinger equation

$$
i \frac{d}{d t}\left(\begin{array}{c}
\nu_{\mu} \\
\nu_{\tau} \\
\bar{\nu}_{\mu} \\
\bar{\nu}_{\tau}
\end{array}\right)=\left(h_{e f f}\right)\left(\begin{array}{c}
\nu_{\mu} \\
\nu_{\tau} \\
\bar{\nu}_{\mu} \\
\bar{\nu}_{\tau}
\end{array}\right)
$$

For definiteness $\tilde{g}^{Z T}$ is assumed to be the only non-zero $\tilde{g}$-type coefficient, where $\tilde{g}$ is defined via

$$
\tilde{g}^{\nu \sigma} \equiv g^{0 \nu \sigma}+\frac{i}{2} \epsilon_{\gamma \rho}^{0 \nu} g^{\gamma \rho \sigma}
$$

Then $b_{\mu}$ and $b_{\tau}$ coefficients are introduced as:

$$
\begin{gathered}
b_{\mu} \equiv 2 \sin \Theta g_{\mu}, \\
b_{\tau} \equiv 2 \sin \Theta g_{\tau} .
\end{gathered}
$$


It is further assumed that $\left(c_{L}\right)_{a b}^{T T}$ and $\left(c_{L}\right)_{a b}^{Z Z}$ are the only nonzero $c_{L}$-type coefficients. The coefficients $\left(c_{L}\right)_{a b}^{T T}$ and $\left(c_{L}\right)_{a b}^{Z Z}$ are, in fact, equal to one another. The superscript TT and subscript L for the $c_{L}$ type coefficients will henceforth be omitted, and $c_{\mu \mu}$ and $c_{\tau \tau}$ coefficients are introduced so that we can write:

$$
\begin{gathered}
c_{\mu \mu}=2\left(c_{L}\right)_{\mu \mu}^{T T}\left[1+\cos ^{2} \Theta\right], \\
c_{\tau \tau}=2\left(c_{L}\right)_{\tau \tau}^{T T}\left[1+\cos ^{2} \Theta\right] .
\end{gathered}
$$

Here $\Theta$ is the celestial colatitude and here it is the angle made by the neutrino beamline with the Z-axis. All $c_{a b}$ 's are assumed to be real. Moreover all contributions from $a_{L}$ and H-type coefficients are neglected. The effective Hamiltonian now reads:

$$
h_{e f f}=\operatorname{diag}\left(E+\frac{\Sigma m^{2}}{4 E}\right)
$$

$$
+\left(\begin{array}{cccc}
\frac{-\Delta m^{2}}{4 E} \cos 2 \theta-\frac{c_{\mu \mu} E}{2} & \frac{\Delta m^{2}}{4 E} \sin 2 \theta & \frac{b_{\mu} E}{2} & 0 \\
\frac{\Delta m^{2}}{4 E} \sin 2 \theta & \frac{\Delta m^{2}}{4 E} \cos 2 \theta-\frac{c_{\tau \tau} E}{2} & 0 & \frac{b_{\tau} E}{2} \\
\frac{b_{\mu} E}{2} & 0 & \frac{-\Delta m^{2}}{4 E} \cos 2 \theta-\frac{c_{\mu \mu} E}{2} & \frac{\Delta m^{2}}{4 E} \sin 2 \theta \\
0 & \frac{b_{\tau} E}{2} & \frac{\Delta m^{2}}{4 E} \sin 2 \theta & \frac{\Delta m^{2}}{4 E} \cos 2 \theta-\frac{c_{\tau \tau} E}{2}
\end{array}\right)
$$

This matrix is symmetric and the eigenvalues of the given Hamiltonian can be interpreted as the effective energy eigenvalues of the system. The effective Hamiltonian Eq. 2.8 is a $4 \times$ 4 matrix, which consists of four $2 \times 2$ block matrices. The CPT-violating $b$-type coefficients generate neutrino-antineutrino mixing, while the CPT-conserving $c$-type coefficients yield Lorentz-violating terms altering the dispersion relations of the flavour states. In the limit 
where the CPT and Lorentz-violating coefficients are set to zero the Hamiltonian which describes the standard neutrino oscillations is retained. It is possible to block-diagonalise the effective Hamiltonian $h_{e f f}$ in terms of a block diagonal effective Hamiltonian $\tilde{h}_{e f f}$ via

$$
h_{e f f}=U \tilde{h}_{e f f} U^{\dagger}
$$

This expression is plugged back into Eq. 2.4 and the basis is changed to

$$
\left(\begin{array}{c}
\nu_{\mu} \\
\nu_{\tau} \\
\bar{\nu}_{\mu} \\
\bar{\nu}_{\tau}
\end{array}\right) \rightarrow \frac{1}{\sqrt{2}}\left(\begin{array}{c}
\nu_{\mu}-\bar{\nu}_{\mu} \\
\nu_{\tau}-\bar{\nu}_{\tau} \\
\nu_{\mu}+\bar{\nu}_{\mu} \\
\nu_{\tau}+\bar{\nu}_{\tau}
\end{array}\right)
$$

$\nu^{-}$and $\nu^{+}$are defined as,

$$
\begin{gathered}
\nu^{-}=\left(\begin{array}{c}
\nu_{\mu}^{-} \\
\nu_{\tau}^{-}
\end{array}\right) \equiv \frac{1}{\sqrt{2}}\left(\begin{array}{c}
\nu_{\mu}-\bar{\nu}_{\mu} \\
\nu_{\tau}-\bar{\nu}_{\tau}
\end{array}\right), \\
\nu^{+}=\left(\begin{array}{c}
\nu_{\mu}^{+} \\
\nu_{\tau}^{+}
\end{array}\right) \equiv \frac{1}{\sqrt{2}}\left(\begin{array}{c}
\nu_{\mu}+\bar{\nu}_{\mu} \\
\nu_{\tau}+\bar{\nu}_{\tau}
\end{array}\right)
\end{gathered}
$$

and the charge conjugation operator $C$ in flavour space is introduced which acts on the different flavour states such that $\nu^{-}$is odd and $\nu^{+}$is even under charge-conjugation. Given these prerequisites the Schrodinger equation for the effective Hamiltonian can be rewritten in a $2 \times 2$ block matrix form:

$$
i \frac{d}{d t}\left(\begin{array}{c}
\nu^{-} \\
\nu^{+}
\end{array}\right)=\left(\begin{array}{cc}
h^{C \text {-odd }} & 0 \\
0 & h^{C \text {-even }}
\end{array}\right)\left(\begin{array}{l}
\nu^{-} \\
\nu^{+}
\end{array}\right)
$$

Since Eq. 2.13 is block diagonal the $C$-odd and $C$-even states decouple and, therefore, 
neutrino-antineutrino oscillations are possible only between the $C$-odd and $C$-even states separately.

\subsection{Directional Dependence}

As mentioned earlier the frame of reference used is the Sun-centered celestial equatorial frame with coordinates ( $\mathrm{T}, \mathrm{X}, \mathrm{Y}, \mathrm{Z})$, illustrated in Fig. 2.1. In this frame the $\mathrm{Z}$ direction is identified as lying along the Earth's rotational axis and the $\mathrm{X}$ direction as pointing towards the vernal equinox. The coefficients for Lorentz violation in any other inertial frame can be related to the standard set in the Sun-centered frame by an observer Lorentz transformation. The local co-ordinates of the experiment are specified in Earth-centered co-ordinates (x, y, z). Finally, local polar co-ordinates $(\theta, \phi)$ are used to specify the direction of the beam.
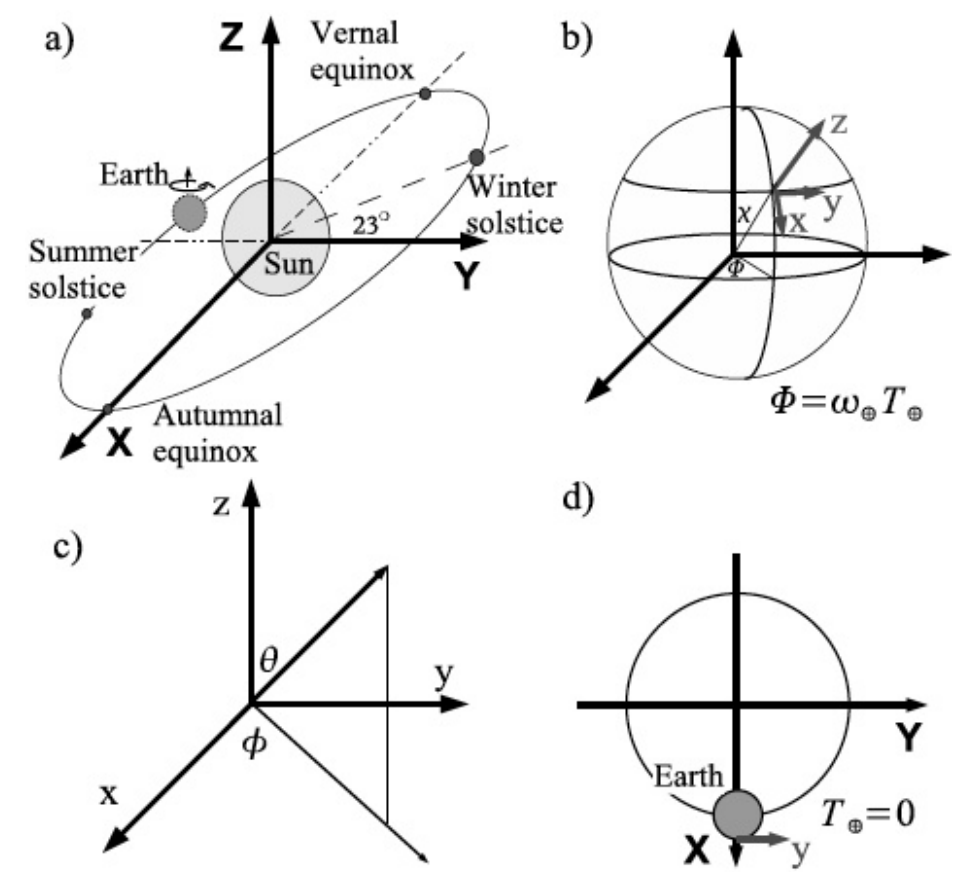

d)

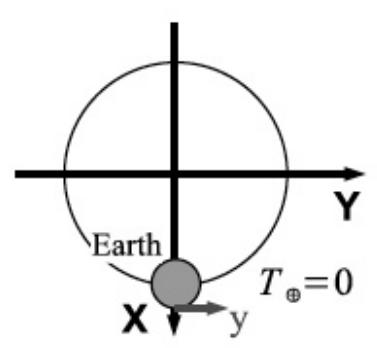

Figure 2.1: Co-ordinate system used for this analysis: a) the Sun-centered system, b) the Earth-centered system, c) the local polar coordinate system, d) the time zero is defined as the location of the experimental site at midnight near autumnal equinox. Figure from [95]. 
A standard parametrisation for the direction of neutrino propagation is

$$
\begin{array}{r}
\hat{p}=(\sin \Theta \cos \Phi, \sin \Theta \sin \Phi, \cos \Theta), \\
\hat{\epsilon}_{1}=(\cos \Theta \cos \Phi, \cos \Theta \sin \Phi,-\sin \Theta), \\
\hat{\epsilon}_{2}=(-\sin \Phi, \cos \Phi, 0),
\end{array}
$$

where $\Theta$ and $\Phi$ are the celestial colatitude and longitude, respectively, and $\hat{p}$ is the unit 3 -vector of the particle's momentum. Therefore in this parametrisation $\Theta$ is the angle made by the neutrino beam with the Earth's rotational axis (Z-axis). It can be seen from Eq. 2.6 that $b_{\mu}$ and $b_{\tau}$ depend on $\sin \Theta$, and can be zero for $\Theta=0$ or $\pi$. This means that for certain orientations of the NuMI beam there would be no observable neutrino-antineutrino mixing even if the $b$-type parameters are non-zero. But since the NuMI beamline is fixed on Earth the value of $\Theta$ stays constant and the oscillations are time independent. A coordinate transformation is performed between Sun-centered co-ordinates and Earth-centered co-ordinates [96] to find $\Theta$ :

$$
\cos \Theta=-\sin \chi \sin \theta \cos \phi+\cos \chi \cos \theta
$$

where $\chi$ is the colatitude of the beamline. For the NuMI beamline $\chi=90^{\circ}$ - latitude $=$ $42.17973347^{\circ}$, the beam zenith angle $\theta=93.2745^{\circ}$ defined from the z-axis which points up towards the local zenith, and the beam azimuthal angle $\phi=203.909^{\circ}$ measured counterclockwise from the x-axis chosen to lie along the detector's long axis. Substituting these values in Eq. 2.15 we calculate $\Theta=55.214^{\circ}$ and therefore, $\sin \Theta=0.8$. Thus the value of $b_{\mu}$ and $b_{\tau}$ is enhanced by a constant factor of 1.6 , and that of $c_{\mu \mu}$ and $c_{\tau \tau}$ is enhanced by a factor 2.6. Thus, we can write

$$
b_{\alpha}=1.6 \tilde{g}_{\alpha \bar{\alpha}}^{Z T}, \text { and } c_{\alpha \alpha}=2.6\left(c_{L}\right)_{\alpha \alpha}^{T T},
$$


where $\alpha$ is $\mu$ or $\tau$.

\subsubsection{Effective Mixing Angle}

The oscillation between $C$-odd and $C$-even states is considered separately. The effective mixing angle for the $C$-odd states can be written in terms of the standard 2-flavour mixing angle $\theta$ and $\Delta m^{2}$ as

$$
\tan 2 \theta_{C-o d d}=\frac{\Delta m^{2} \sin 2 \theta}{\left(\left(b_{\mu}-b_{\tau}+c_{\mu \mu}-c_{\tau \tau}\right) E^{2}+\Delta m^{2} \cos 2 \theta\right)}
$$

and that between the $C$-even states as

$$
\tan 2 \theta_{\text {C-even }}=\frac{\Delta m^{2} \sin 2 \theta}{\left(\left(-b_{\mu}+b_{\tau}+c_{\mu \mu}-c_{\tau \tau}\right) E^{2}+\Delta m^{2} \cos 2 \theta\right)}
$$

\subsubsection{Conditions for Resonance}

The conditions to be satisfied for resonant mixing are

$$
b_{\mu}-b_{\tau}>\left|c_{\mu \mu}-c_{\tau \tau}\right| \Rightarrow \text { only } C \text {-even resonance, }
$$

$$
b_{\mu}-b_{\tau}<-\left|c_{\mu \mu}-c_{\tau \tau}\right| \Rightarrow \text { only } C \text {-odd resonance, }
$$

If $\operatorname{sgn}\left(c_{\mu \mu}-c_{\tau \tau}\right)=-1$, then for

$$
-\left|c_{\mu \mu}-c_{\tau \tau}\right|<b_{\mu}-b_{\tau}<\left|c_{\mu \mu}-c_{\tau \tau}\right| \Rightarrow \text { both resonances exist }
$$

whereas if $\operatorname{sgn}\left(c_{\mu \mu}-c_{\tau \tau}\right)=+1$, then for

$$
-\left|c_{\mu \mu}-c_{\tau \tau}\right|<b_{\mu}-b_{\tau}<\left|c_{\mu \mu}-c_{\tau \tau}\right| \Rightarrow \text { neither of the resonances exists. }
$$


It is seen that the oscillation probability $\nu_{\mu}$ to $\bar{\nu}_{\mu}$ is maximum when either C-odd or C-even resonance exists. For the case when both C-even and C-odd states exist this probability is much smaller, and is zero when none of the resonance states exists. This will be explained in detail in Section 2.4.

\subsection{Oscillation Probability}

The oscillation probabilities are calculated using the matrix $\mathrm{V}([94])$ which diagonalises the effective Hamiltonian and translates the flavour eigenstates $\nu_{\mu}, \nu_{\tau}, \bar{\nu}_{\mu}$, and $\bar{\nu}_{\tau}$ into the mass eigenbasis $\nu_{1}, \nu_{2}, \nu_{3}$, and $\nu_{4}$. The matrix $\mathrm{V}$ is written as:

$$
V=\frac{1}{\sqrt{2}}\left(\begin{array}{cccc}
\cos \theta_{C \text {-odd }} & \sin \theta_{C \text {-odd }} & \cos \theta_{C \text {-even }} & \sin \theta_{C \text {-even }} \\
-\sin \theta_{C \text {-odd }} & \cos \theta_{C \text {-odd }} & -\sin \theta_{C \text {-even }} & \cos \theta_{C \text {-even }} \\
-\cos \theta_{C \text {-odd }} & -\sin \theta_{C \text {-odd }} & \cos \theta_{C \text {-even }} & \sin \theta_{C \text {-even }} \\
\sin \theta_{C \text {-odd }} & -\cos \theta_{C \text {-odd }} & -\sin \theta_{C \text {-even }} & \cos \theta_{C \text {-even }}
\end{array}\right)
$$

and the effective hamiltonian $h_{e f f}^{\text {diag }}$ is obtained via,

$$
h_{e f f}^{\operatorname{diag}}=V^{\dagger} h_{e f f} V .
$$

The oscillation probability is obtained by squaring the amplitude of oscillation,

$$
P(\beta \rightarrow \alpha)=\left|\sum_{i} V_{\beta i} e^{-i E_{i} t}\left(V_{i \alpha}^{\dagger}\right)\right|^{2}
$$

where $E_{i}$ are the effective energy eigenvalues of the associated Hamiltonian $h_{\text {eff }} ; \alpha$ and $\beta$ stand for the four neutrino species involved: $\nu_{\mu}, \nu_{\tau}, \bar{\nu}_{\mu}, \bar{\nu}_{\tau}$. Considering $\Delta m^{2}$ to be the mass-squared difference between the mass eigenstates $\nu_{1}$ and $\nu_{2}$, or $\nu_{3}$ and $\nu_{4}$, the various oscillation probabilities are given by: 


\section{Survival probability of $\nu_{\mu}$ :}

$$
P\left(\nu_{\mu} \rightarrow \nu_{\mu}\right)=1-\left(\sin ^{2}\left(\theta_{\text {odd }}-\theta_{\text {even }}\right)+\frac{1}{4}\left[\sin \left(2 \theta_{\text {odd }}\right)+\sin \left(2 \theta_{\text {even }}\right)\right]^{2}\right) \sin ^{2}\left(\frac{\Delta m^{2} L}{4 E}\right)
$$

Oscillation probability $\nu_{\mu}$ to $\bar{\nu}_{\mu}$ :

$$
P\left(\nu_{\mu} \rightarrow \bar{\nu}_{\mu}\right)=\left(\sin ^{2}\left(\theta_{\text {odd }}-\theta_{\text {even }}\right)-\frac{1}{4}\left[\sin \left(2 \theta_{\text {odd }}\right)-\sin \left(2 \theta_{\text {even }}\right)\right]^{2}\right) \sin ^{2}\left(\frac{\Delta m^{2} L}{4 E}\right)
$$

Oscillation probability $\nu_{\mu}$ to $\nu_{\tau}$ :

$$
P\left(\nu_{\mu} \rightarrow \nu_{\tau}\right)=\left(\frac{1}{4}\left[\sin \left(2 \theta_{\text {odd }}\right)+\sin \left(2 \theta_{\text {even }}\right)\right]^{2}\right) \sin ^{2}\left(\frac{\Delta m^{2} L}{4 E}\right)
$$

Oscillation probability $\nu_{\mu}$ to $\bar{\nu}_{\tau}$ :

$$
P\left(\nu_{\mu} \rightarrow \bar{\nu}_{\tau}\right)=\left(\frac{1}{4}\left[\sin \left(2 \theta_{\text {odd }}\right)-\sin \left(2 \theta_{\text {even }}\right)\right]^{2}\right) \sin ^{2}\left(\frac{\Delta m^{2} L}{4 E}\right)
$$

If the Lorentz and CPT-violating parameters are zero, the expressions for the oscillation probabilities Eq. 2.26 and 2.28, respectively, reduce to the standard oscillation probabilities:

$$
\begin{gathered}
P\left(\nu_{\mu} \rightarrow \nu_{\mu}\right)=1-\sin ^{2} 2 \theta \sin ^{2}\left(\Delta m^{2} \frac{L}{4 E}\right), \text { and } \\
P\left(\nu_{\mu} \rightarrow \nu_{\tau}\right)=\sin ^{2} 2 \theta \sin ^{2}\left(\Delta m^{2} \frac{L}{4 E}\right)
\end{gathered}
$$


Furthermore, the neutrino to antineutrino oscillation probabilities Eq. 2.27 and 2.29 become zero if the Lorentz and CPT-violating parameters are zero.

From the resonance conditions mentioned in Section 2.3 .2 it is clear that there is an ambiguity in the model because of which one cannot distinguish between the cases when the parameters are actually small and when they are large but don't satisfy the resonance condition. Therefore to discuss some of the features of this model it is assumed that the coefficients $\left(c_{L}\right)_{\mu \mu}^{T T}$ and $\left(c_{L}\right)_{\tau \tau}^{T T}$ are zero, and because of degeneracy between the parameters $\tilde{g}_{\mu \bar{\mu}}^{Z T}$ and $\tilde{g}_{\tau \bar{\tau}}^{Z T}$ any one of these two parameters is considered (these are relabelled $\tilde{g}_{\alpha \bar{\alpha}}^{Z T}$, where $\alpha$ can be $\mu$ or $\tau$ ), assuming the other one to be zero. Then the condition for either the C-odd or C-even resonance will always be satisfied for any non-zero value of $\tilde{g}_{\alpha \bar{\alpha}}^{Z T}$. It can be seen from Eq. 2.17 and 2.18 that there is a threshold in energy above which the Lorentz and CPT violating parameters dominate. This threshold is roughly given by $\mathrm{E} \sim \sqrt{\Delta m^{2} / 1.6 \mid \tilde{g}_{\alpha \bar{\alpha}}^{Z T}}$ GeV. Fig. 2.2 shows the variation of effective mixing angles $\theta_{C \text {-odd }}$ and $\theta_{C \text {-even }}$ plotted against E. The angle $\theta_{C \text {-odd }}$ and $\theta_{C \text {-even }}$ approaches 0 and $\pi / 2$, respectively, for $\mathrm{E} \gg \sqrt{\Delta m^{2} / 1.6 \mid \tilde{g}_{\alpha \bar{\alpha}}^{Z T}}$ $\mathrm{GeV}$. Therefore above this threshold the oscillations approach maximal mixing and do not depend on the value of $\theta_{23}$. Assuming $\tilde{g}_{\tau \bar{\tau}}^{Z T}=0$, for $\tilde{g}_{\mu \bar{\mu}}^{Z T}>0$ the resonance condition for the existence of only the $C$-even states is satisfied and $\tilde{g}_{\mu \bar{\mu}}^{Z T}<0$ satisfies the resonance condition for $C$-odd states. On the other hand, assuming $\tilde{g}_{\mu \bar{\mu}}^{Z T}=0$, the conditions for $C$-odd and $C$-even resonances will be reversed; the oscillation probabilities would be unaffected.

The same energy threshold changes the oscillation mode from $\nu_{\mu} \rightarrow \nu_{\tau}$ to $\nu_{\mu} \rightarrow \bar{\nu}_{\mu}$. For small $\tilde{g}_{\alpha \bar{\alpha}}^{Z T}$ the threshold is at higher energies where no oscillations occur. As $\tilde{g}_{\alpha \bar{\alpha}}^{Z T}$ increases the threshold moves to lower energies and $\nu_{\mu} \rightarrow \bar{\nu}_{\mu}$ appearance is seen instead of $\nu_{\mu} \rightarrow \nu_{\tau}$ disappearance [98]. However $\tilde{g}_{\alpha \bar{\alpha}}^{Z T}$ doesn't change the oscillation frequency.

Fig. 2.3 and Fig. 2.4. respectively, show the oscillation probabilities $\nu_{\mu} \rightarrow \bar{\nu}_{\mu}$ and $\nu_{\mu} \rightarrow$ $\bar{\nu}_{\tau}$ for the value of $\Delta m^{2}=2.32 \times 10^{-3} \mathrm{eV}^{2}, \sin ^{2} 2 \theta=0.97, \tilde{g}_{\alpha \bar{\alpha}}^{Z T}=2 \times 10^{-22}$. Fig. 2.3 shows a finite probability of $\nu_{\mu} \rightarrow \bar{\nu}_{\mu}$ as well as $\nu_{\mu} \rightarrow \bar{\nu}_{\tau}$ oscillations at $\sim 2 \mathrm{GeV}$ while Fig. 2.4 compares the $\nu_{\mu} \rightarrow \nu_{\mu}$ survival and $\nu_{\mu} \rightarrow \nu_{\tau}$ oscillation probabilities using the SME model 

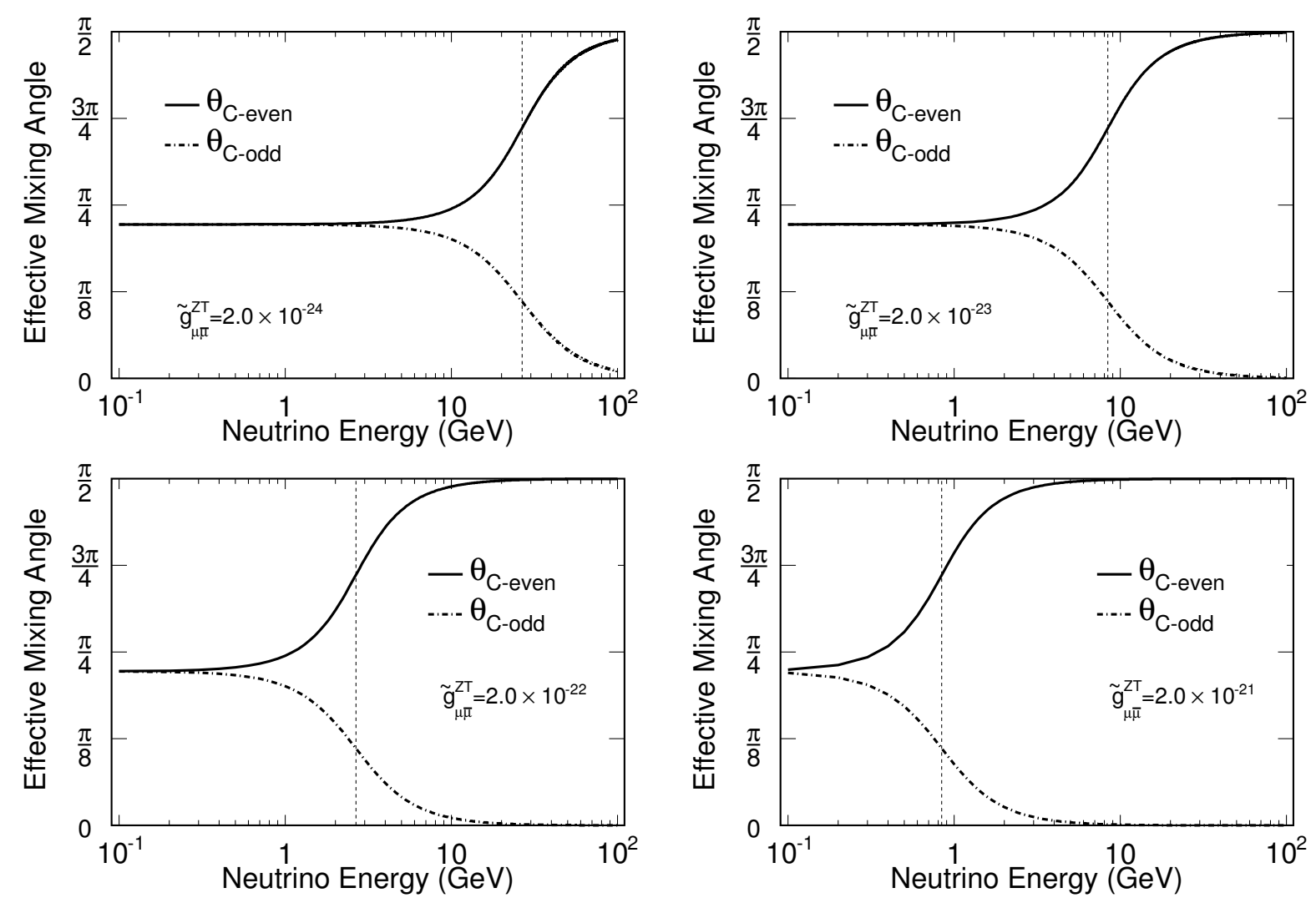

Figure 2.2: The plot shows the effective mixing angle, $\theta_{C \text {-odd }}$ or $\theta_{C \text {-even }}$ as a function of neutrino energy $E$. We have chosen $\Delta m^{2}=2.32 \times 10^{-3} \mathrm{eV}^{2}, \sin ^{2} 2 \theta=0.97$ (values taken from [97]), and four different values of $\tilde{g}_{\mu \bar{\mu}}^{Z T} \cdot \tilde{g}_{\mu \bar{\mu}}^{Z T} \cdot\left(c_{L}\right)_{\mu \mu}^{T T}$, and $\left(c_{L}\right)_{\tau \tau}^{T T}$ are assumed to be zero. The dotted vertical line denoting the energy threshold is drawn at $E=\sqrt{\Delta m^{2} / 1.6\left|\tilde{g}_{\mu \bar{\mu}}^{Z T}\right|}$ $\mathrm{GeV}$. We can see the threshold decreases as the value of $\tilde{g}_{\mu \bar{\mu}}^{Z T}$ is increased. 

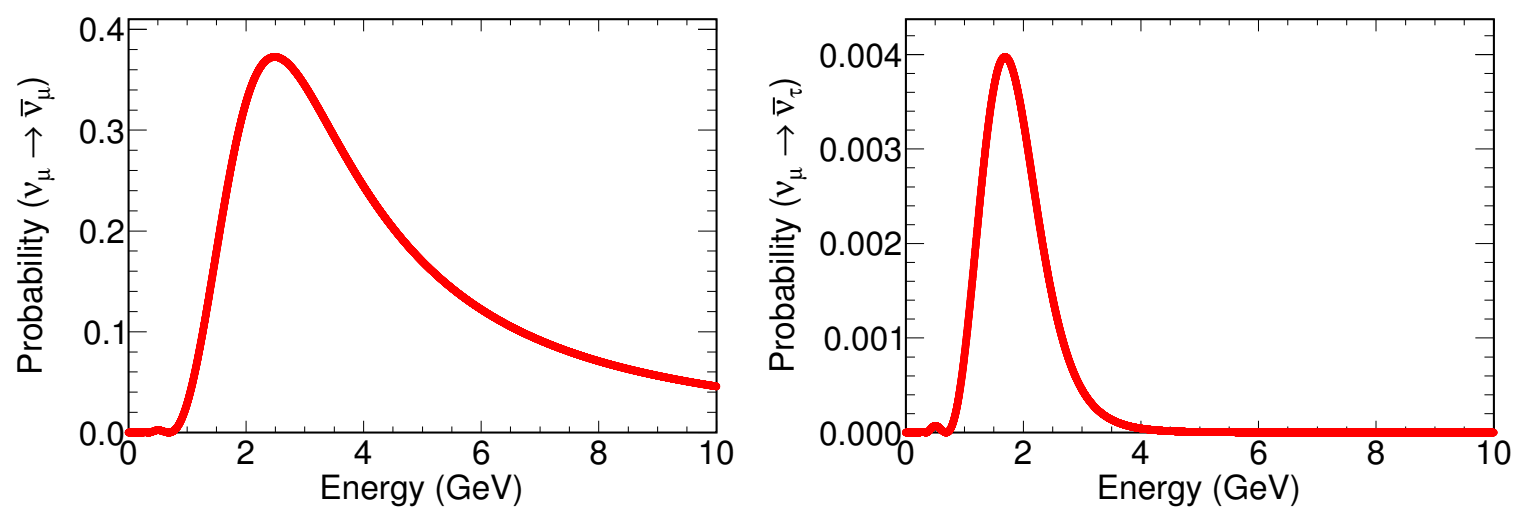

Figure 2.3: Oscillation probabilities for $\nu_{\mu} \rightarrow \bar{\nu}_{\mu}$ and $\nu_{\mu} \rightarrow \bar{\nu}_{\tau}$. $\left(\tilde{g}_{\alpha \bar{\alpha}}^{Z T}=2 \times 10^{-22}, \Delta m^{2}=\right.$ $\left.2.32 \times 10^{-3} \mathrm{eV}^{2}, \sin ^{2} 2 \theta=0.97\right)$
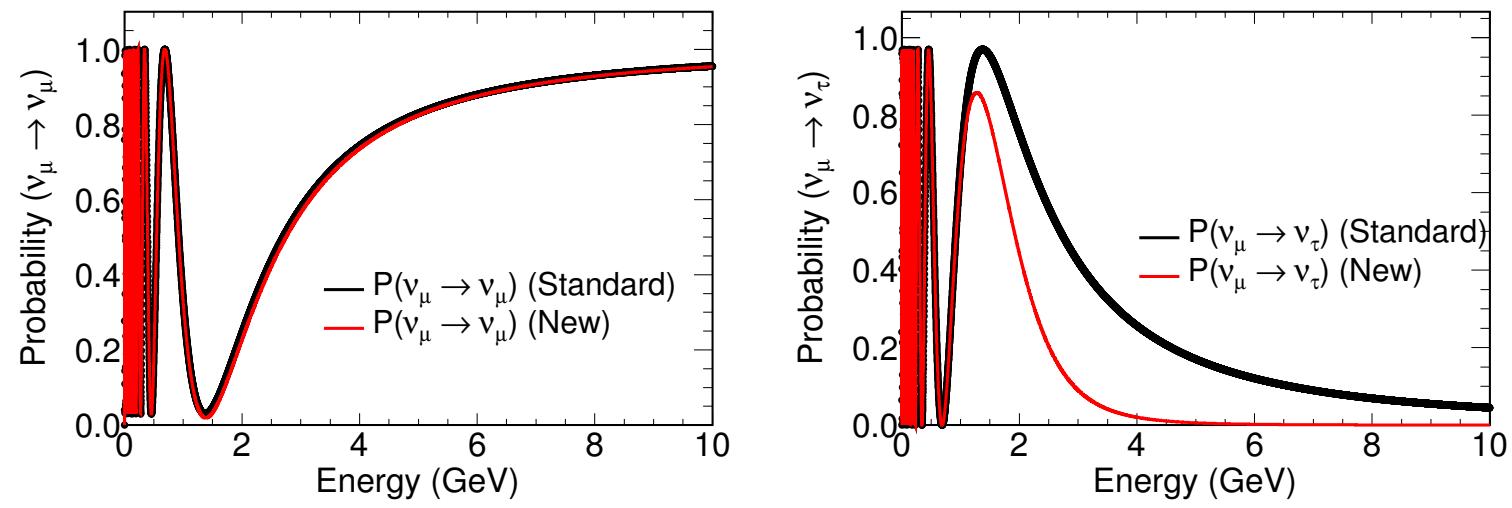

Figure 2.4: Probabilities for $\nu_{\mu} \rightarrow \nu_{\mu}$ survival and $\nu_{\mu} \rightarrow \nu_{\tau}$ oscillation as a function of neutrino energy. The red line shows the oscillation probabilities using the new model with $\tilde{g}_{\alpha \bar{\alpha}}^{Z T}=2 \times 10^{-22}$ and the black line shows the oscillation probabilities using the standard two-flavour oscillations model $\left(\Delta m^{2}=2.32 \times 10^{-3} \mathrm{eV}^{2}\right.$ and $\sin ^{2} 2 \theta=0.97$ in both cases $)$.

and the standard two-flavour oscillation model.

\subsection{Summary}

From equation 2.3 it can be seen that $\left(b_{\mu}-b_{\tau}\right)=1.6 \times\left(\tilde{g}_{\mu \bar{\mu}}^{Z T}-\tilde{g}_{\tau \bar{\tau}}^{Z T}\right)$, and $\left(c_{\mu \mu}-c_{\tau \tau}\right)=$ $2.6 \times\left(\left(c_{L}\right)_{\mu \mu}^{T T}-\left(c_{L}\right)_{\tau \tau}^{T T}\right)$. Since $\tilde{g}_{\mu \bar{\mu}}^{Z T}$ and $\tilde{g}_{\tau \bar{\tau}}^{Z T}$ are degenerate, we are only sensitive to the difference $\left(\tilde{g}_{\mu \bar{\mu}}^{Z T}-\tilde{g}_{\tau \bar{\tau}}^{Z T}\right)$; and by the same reasoning to $\left(\left(c_{L}\right)_{\mu \mu}^{T T}-\left(c_{L}\right)_{\tau \tau}^{T T}\right)$. So we attempt to constrain this combination of parameters by performing a fit to MINOS data. Since the sign of the g-type parameters does not affect the oscillation probabilities we can constrain only 
the magnitude of these parameters. Non-zero values of $\tilde{g}$-type coefficients lead to mixing between neutrinos and antineutrinos, therefore the SME parameters $\left(\tilde{g}_{\mu \bar{\mu}}^{Z T}-\tilde{g}_{\tau \bar{\tau}}^{Z T}\right)$ can be obtained by searching for an excess of antineutrino events at the Far Detector as compared to the number of events expected from the standard two-flavour oscillations. In addition, $\left(\left(c_{L}\right)_{\mu \mu}^{T T}-\left(c_{L}\right)_{\tau \tau}^{T T}\right)$ also lead to a change in the oscillations in the standard $\nu_{\mu}-\nu_{\tau}$ sector. Oscillations in the presence of the SME coefficients in the present model are time independent and the energy at which $\nu_{\mu}$ to $\bar{\nu}_{\mu}$ oscillations occur depends on the size of the parameters. Furthermore, the constraint on individual SME parameters can also be obtained by setting all other parameters to zero, and fitting for only that parameter. This analysis will help to increase our knowledge of Lorentz and CPT violation in the neutrino sector. 


\section{Chapter 3}

\section{The MINOS Beam and Detectors}

The Main Injector Neutrino Oscillation Search (MINOS) is a long-baseline neutrino-oscillation experiment. It is made up of the NuMI (Neutrinos at the Main Injector) high intensity neutrino beam and two detectors. The NuMI beam is located at Fermilab, where $120 \mathrm{GeV}$ protons from the Main Injector are directed at a $940 \mathrm{~mm}$ long segmented graphite target to produce mesons. The mesons then decay via weak interactions to produce the neutrino or antineutrino beam. MINOS measures neutrino energy spectrum at the Near Detector (ND) at Fermilab and again $735 \mathrm{~km}$ downstream with a Far Detector (FD) in the Soudan Mine in northern Minnesota. Fig. 3.1 gives a view of the location of the MINOS detectors. Comparison of the neutrino energy spectra at the two detectors allows the measurement of neutrino oscillation parameters.

The two-detector design makes the measurement less dependent on simulations and allows the systematic uncertainties which affect both detectors in the same way to effectively cancel out.

\subsection{The NuMI Beam}

The Neutrinos at the Main Injector (NuMI) facility at Fermilab produces a neutrino beam using protons from the Main Injector (MI). Protons of $120 \mathrm{GeV}$ are extracted from 


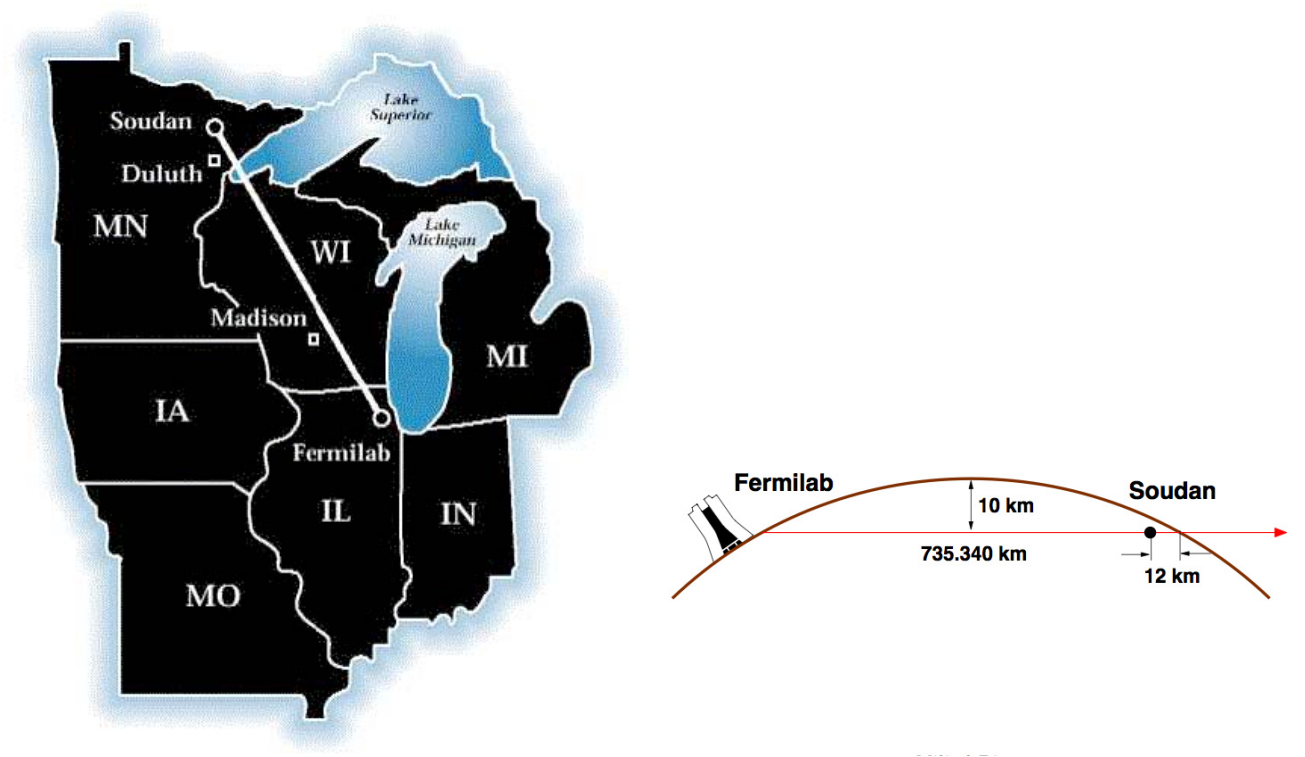

Figure 3.1: The layout of the MINOS Experiment showing the location of the Near and Far Detectors, and the distance between them.

the MI accelerator in a series of pulses, also called spills. Each of these spills contains six batches. Before the Tevatron shutdown on September 30, 2011 NuMI received five or six batches with the remaining one going to produce antiprotons for the Tevatron. After the Tevatron shutdown all six spills are received by NuMI. A spill of protons arrives on average every 2.2 seconds and lasts for $8-10 \mu \mathrm{s}$. At the beginning of the experiment in 2005, each spill consisted of approximately $2.2 \times 10^{13}$ protons on target (POT) with a linear increase to $3.6 \times 10^{13}$ POT at the end of data taking on April 30, 2012. The average beam power is approximately $340 \mathrm{~kW}$. The NuMI beamline is illustrated in Fig. 3.2 and more details can be found in [99, 100] and [101]. The proton beam is aimed $58 \mathrm{mrad}$ downwards into the Earth using quadrupoles and bending magnets to point at the FD, which is situated in the Soudan Mine. The global positioning system (GPS) defines the beam direction to within $12 \mathrm{~m}$ of the FD.

The beam is allowed to strike a rectangular graphite target. The target consists of 47 segments which are $6.4 \mathrm{~mm}$ in width, $15 \mathrm{~mm}$ in height and $20 \mathrm{~mm}$ in length. There is a 
$0.3 \mathrm{~mm}$ spacing between each segment. This gives the target a total length of $954 \mathrm{~mm}$ which corresponds to 1.9 interaction lengths. The beam size at the target is $1.2-1.5 \mathrm{~mm}$. The target shape and dimensions are designed to obtain a high flux of mesons while minimising the number of mesons re-interacting in the target. The target is water cooled by stainless steel tubes at the top and bottom of each segment.

The interaction of protons with the graphite target produces a variety of daughter particles including charged mesons (pions and kaons).

$$
p+C \rightarrow \pi^{ \pm}, K^{ \pm}+X
$$

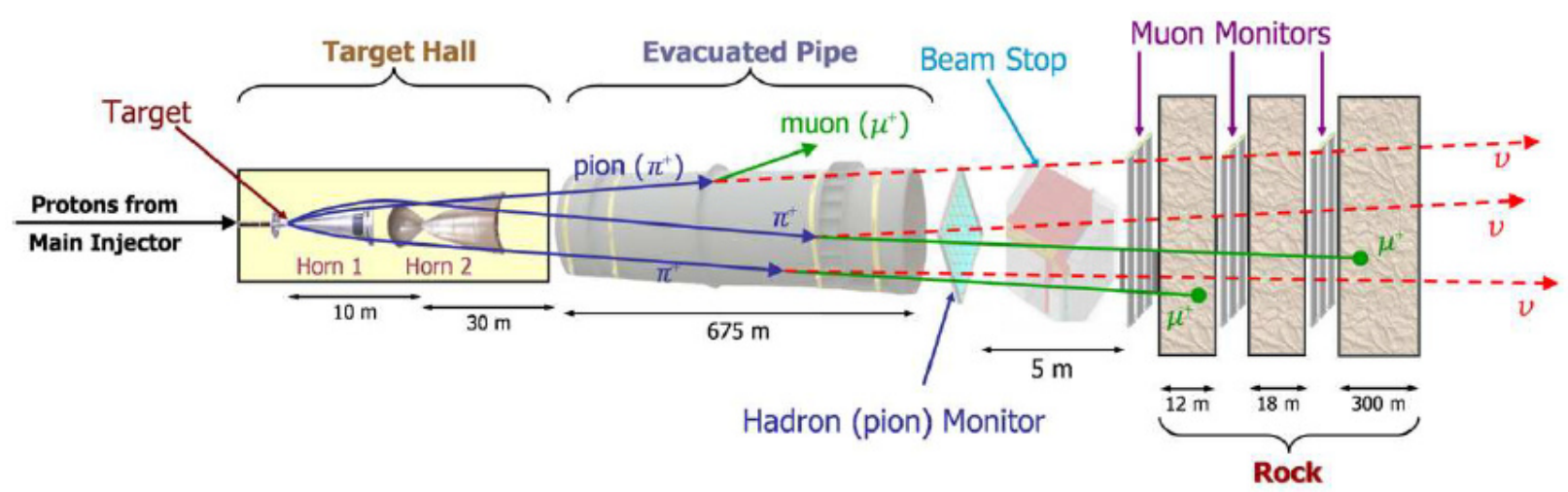

Figure 3.2: Components of the NuMI beamline. Protons of $120 \mathrm{GeV}$ from FNAL Main Injector enter from the left. Figure from [101].

These particles are focused by two magnetic horns that produce a toroidal magnetic field about an axis along the beam direction. These horns are magnetised in such a way as to focus positively charged mesons which will produce muon neutrinos :

$$
\begin{aligned}
& \pi^{+(-)} \rightarrow \mu^{+(-)}+\nu_{\mu}\left(\bar{\nu}_{\mu}\right), \\
& K^{+(-)} \rightarrow \mu^{+(-)}+\nu_{\mu}\left(\bar{\nu}_{\mu}\right) .
\end{aligned}
$$

The focused beam of particles enters a $675 \mathrm{~m}$ long and 2 meters in diameter steel pipe 
where the mesons decay into a muon and a muon neutrino. The length of the decay pipe is approximately the decay length of a $10 \mathrm{GeV}$ pion. The entrance to the decay pipe is sealed by a two-piece aluminium-steel window. The central (radius $<50 \mathrm{~cm}$ ) portion of the window is made of $1 \mathrm{~mm}$ thick aluminium and is strengthened by an outer (radius $>50 \mathrm{~cm}$ ) section made of $1.8 \mathrm{~cm}$ thick steel. For the first two runs of the experiment the decay pipe was evacuated down to about 1 Torr. After that (December 2007) the decay pipe was filled with Helium gas at 0.9 atm. This was done because the strength of the upstream end of the decay pipe had been reduced by the acidic conditions created by the beam, raising concerns of implosion.

The beam goes through a hadron absorber following the decay pipe where leftover mesons and protons get absorbed. The muons range out to $240 \mathrm{~m}$ of rock after the absorber where they, too, get stopped. There is one hadron monitor directly upstream of the hadron absorber and three muon monitors, placed in the rock that follows the absorber, alternating with layers of rock. The beam then reaches the ND. In this forward horn current (FHC) focusing mode the beam at the ND is composed of $91.7 \% \nu_{\mu}, 7.0 \% \bar{\nu}_{\mu}$ and $1.3 \%\left(\nu_{e}+\bar{\nu}_{e}\right)$.

Antineutrinos are produced by the decay of pions and kaons produced when primary protons collide with the graphite target.

$$
p+C \rightarrow \pi^{-} \rightarrow \mu^{-}+\bar{\nu}_{\mu}
$$

The antineutrinos in the $\mathrm{FHC}$ beam come primarily from low- $\mathrm{p}_{\mathrm{T}}$ pions which do not get defocused by the magnetic horns and travel down the axis of the beamline. These pions are called "neck-to-neck" since they pass directly through the necks of both horns. Fig. 3.3 shows the comparison of the neutrino and antineutrino parents' $\mathrm{p}_{\mathrm{T}}-\mathrm{p}_{\mathrm{Z}}$ distributions. Since the "neck-to-neck" parents do not have the advantage of momentum selection by the horns the antineutrinos from these parents have a broader spectrum with a higher peak energy than the neutrinos. So the peak energy of antineutrinos is around $8 \mathrm{GeV}$ instead of $3 \mathrm{GeV}$ 
for neutrinos. The ND spectra of muon neutrinos and antineutrinos in FHC-mode are shown in Fig. 3.4.
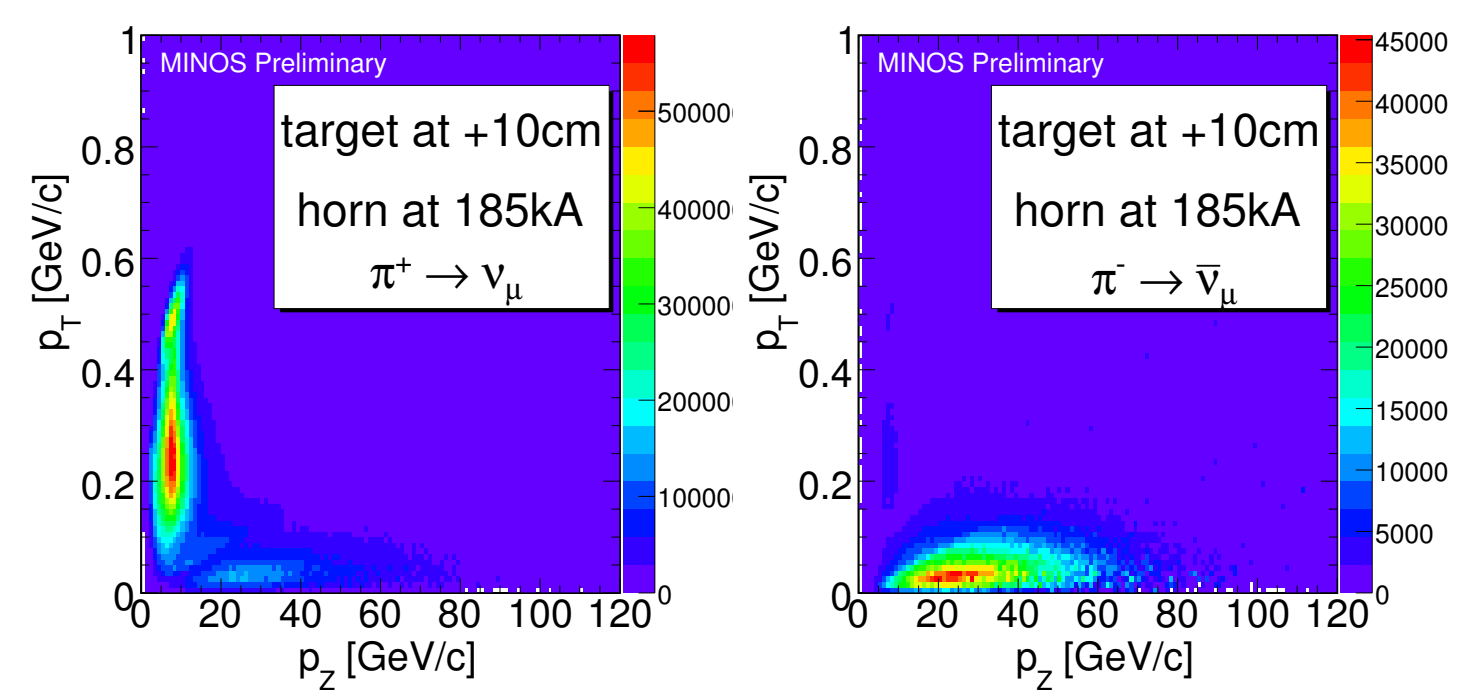

Figure 3.3: The $\mathrm{p}_{\mathrm{T}}$ vs. $\mathrm{p}_{\mathrm{Z}}$ distribution of the $\pi^{ \pm}$parents that produce neutrinos (left) and antineutrinos (right) at the ND when the beam is in low-energy FHC mode.

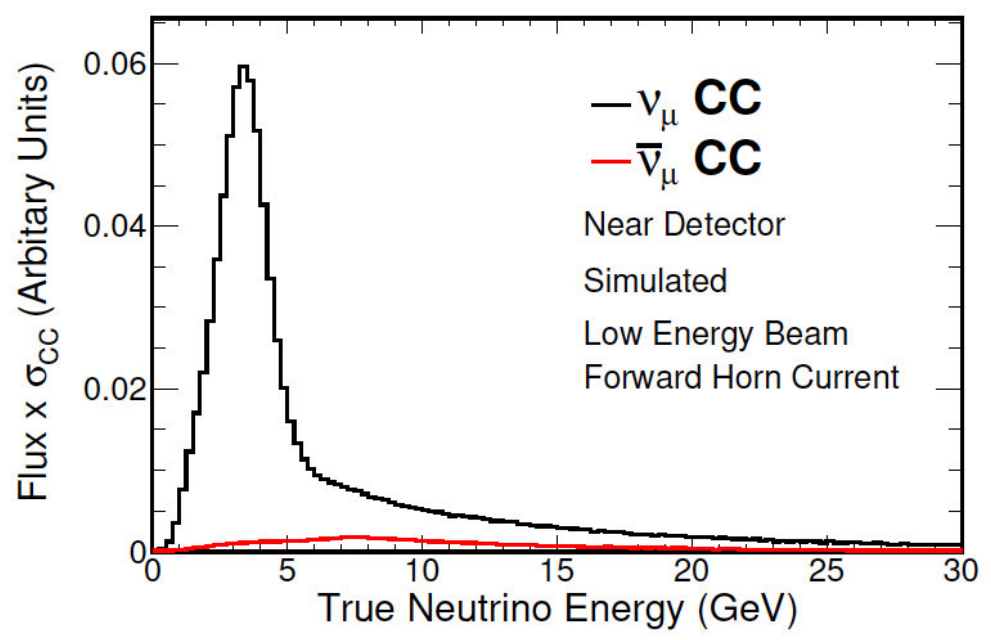

Figure 3.4: Near detector spectra of muon neutrinos and antineutrinos in FHC-mode.

In order to make an antineutrino beam, the polarity of the horn current is reversed to focus negatively charged mesons which decay into antineutrinos. The composition of the reversed horn current (RHC) beam integrated over all energies is $39.9 \% \bar{\nu}_{\mu}, 58.1 \% \nu_{\mu}$ and $2.0 \%\left(\nu_{e}+\bar{\nu}_{e}\right)$. The spectra in this mode are shown in Fig. 3.5 . 
Reversing the horn polarity changes the spectra of signal and background because of the combination of two effects; first, the beam is produced by protons which results in a larger flux of positively charged mesons and second, the interaction cross section of antineutrinos is about half that of neutrinos [102, 103]. This results in a smaller flux compared to the neutrino-mode.

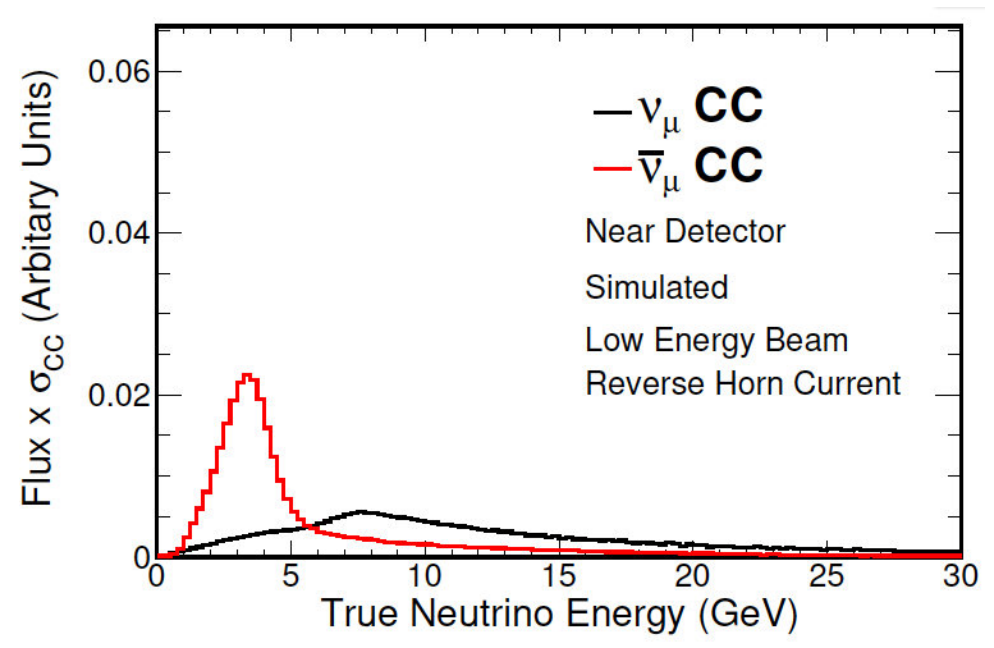

Figure 3.5: Near detector spectra of muon neutrinos and antineutrinos in RHC-mode.

The NuMI beamline can be operated in multiple configurations. The peak energy of the neutrino beam can be adjusted by varying the target position relative to the horns. Fig. 3.6 shows the three configurations that result in low, medium and high energy beams. (All of the data used for physics analysis in this thesis was collected in the "LE-10" configuration where the separation between the horns is $10 \mathrm{~m}$ and the horn current is $185 \mathrm{kA}$.)

\subsection{The MINOS Detectors}

The two MINOS detectors are designed to be as functionally equivalent as possible to minimise the effect of a range of systematic uncertainties. Both the detectors are magnetised steel and scintillator tracking calorimeters with alternating views of scintillator. The detectors are shown in Fig. 3.7 and a schematic of the steel and scintillator planes is shown 

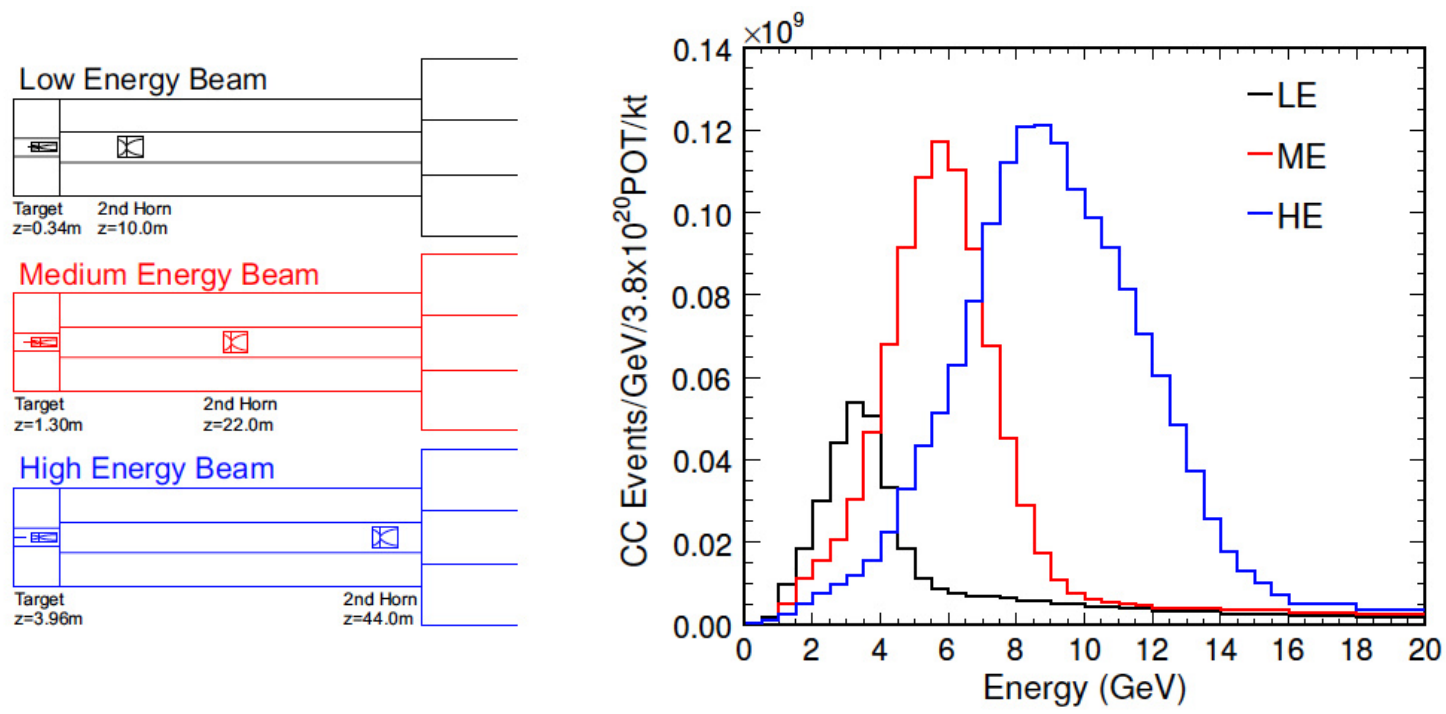

Figure 3.6: Three possible configurations of the NuMI beam (low, medium and high energy: LE, ME and HE). The relative target and focusing horn positions are shown on the left and the corresponding energy spectra on the right.

in Fig. 3.8, Both detectors are made up of alternating layers of $2.54 \mathrm{~cm}$ thick steel and $1 \mathrm{~cm}$ thick scintillator strips of $4.1 \mathrm{~cm}$ width. An air gap makes the total plane separation up to $5.95 \mathrm{~cm}$. The scintillator strips are aligned perpendicular to one another at \pm 45 degrees in $U$ and $V$ views to allow three dimensional reconstruction.
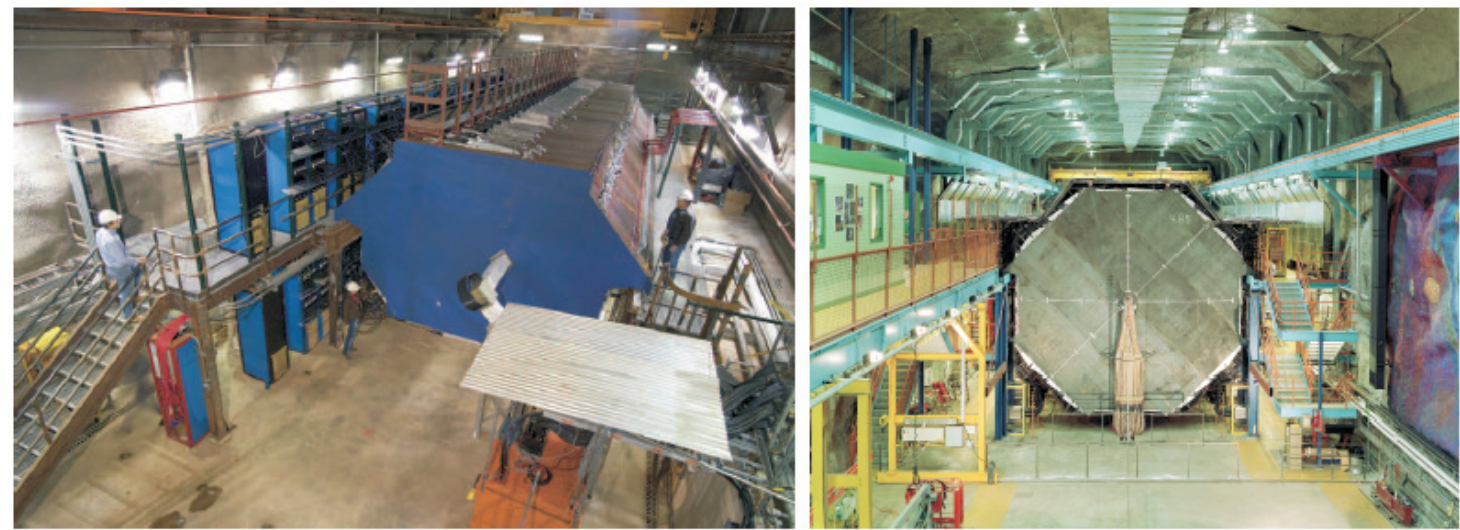

Figure 3.7: The MINOS Near (left) and Far (right) detectors. 


\subsubsection{Near Detector}

The ND is located $110 \mathrm{~m}$ underground at Fermilab. It is a $4.8 \mathrm{~m}$ wide, $3.8 \mathrm{~m}$ high squashed octagon with a length of $16.6 \mathrm{~m}$. It consists of 282 steel planes and has a mass of 980 tons. The first 120 planes (except the first) are instrumented with scintillator. Of these, every fifth plane is fully covered with scintillator and the other four are partially covered. Of the remaining planes, every fifth plane is fully covered with scintillator, the intermediate four planes having no scintillator at all. See Fig. 3.9 for the cross section of a ND plane.

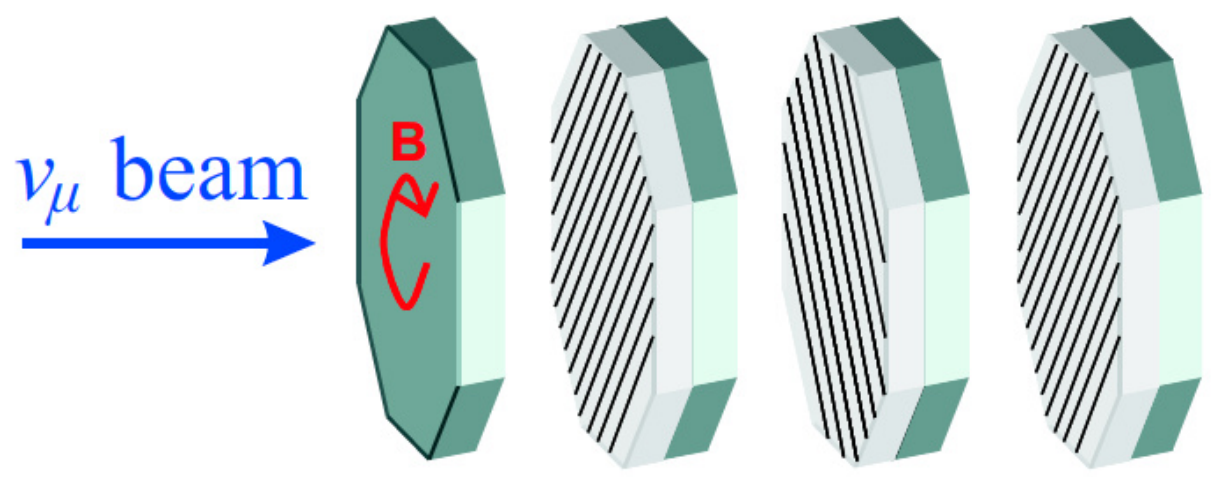

Figure 3.8: A schematic of a MINOS detector showing the scintillator strips and the darker steel planes.

The detector is designed such that the upstream part of the ND is more finely instrumented, and is used for hadronic shower energy measurements. This part of the detector is called the calorimeter. The partially instrumented second half of the detector, called the spectrometer, is used for muon tracking.

The detector is magnetised with a magnetic field of $\sim 1.3 \mathrm{~T}$. The magnetic field focuses the particles of one charge sign and defocuses the other and allows the reconstruction of the charge of a particle travelling through the detector. The curvature of a charged particle is also used to reconstruct its momentum. The beam axis is displaced $1.48 \mathrm{~m}$ horizontally from the coil, in a region where the magnetic field is similar to that in the FD, so as to minimise the number of tracks crossing the coil hole region. 


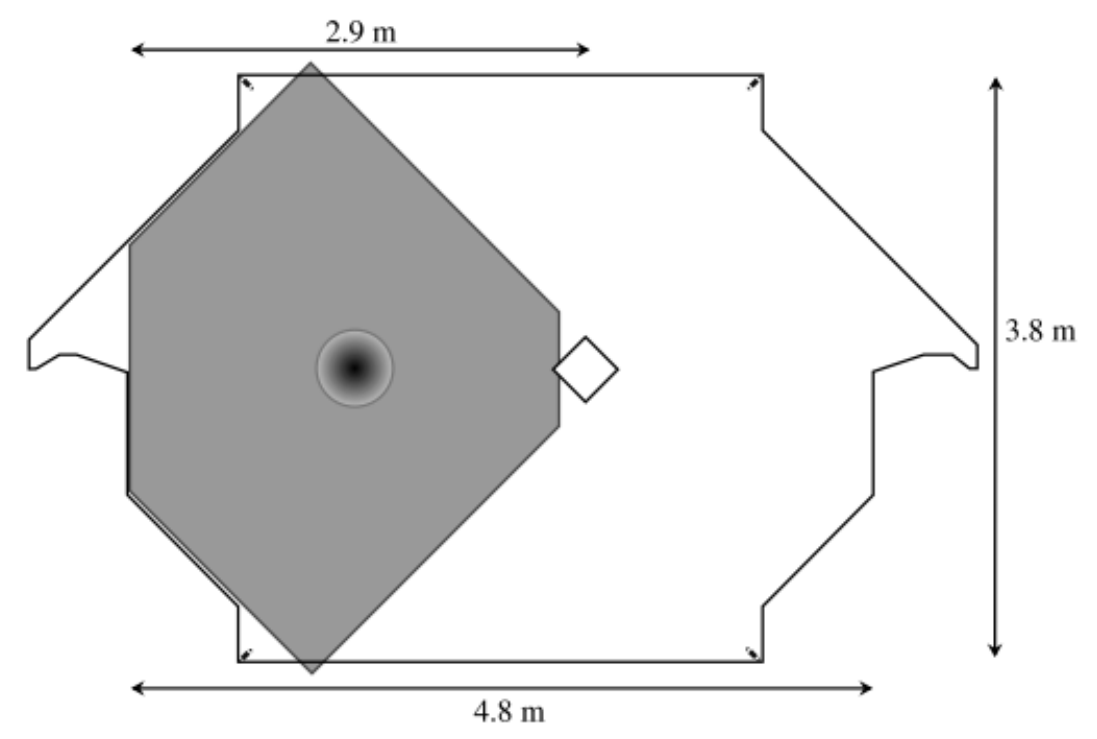

Figure 3.9: The cross-section of a MINOS ND plane. The grey area is covered with scintillator in the partially instrumented planes, the entire plane being covered in the fully instrumented planes. The central diamond shows the position of the coil. The dark circle marks the centre of the neutrino beam.

\subsubsection{Far Detector}

The FD consists of 486 octagonal steel planes $8 \mathrm{~m}$ across. It has a length of $31 \mathrm{~m}$ and a mass of 5.4 kton. The FD is split into two supermodules of 249 and 237 planes, separated by a gap of approximately $1.2 \mathrm{~m}$. The event rate at the FD is of the order of a few events per day, in contrast to approximately ten interactions per spill at the ND. It is located at a depth of about $705 \mathrm{~m}$ in the Soudan mine, to reduce the cosmic ray background. Cosmic rays are also vetoed by a veto shield covering the top of the detector along its length. Each plane is fully covered with 192 strips of scintillator, except the front plane of each supermodule. The FD is also magnetised with an average magnetic field of $\sim 1.4 \mathrm{~T}$, similar to that in the ND. The Near and Far Detector scintillator module arrangements are shown in figures 3.10 and 3.11 . 

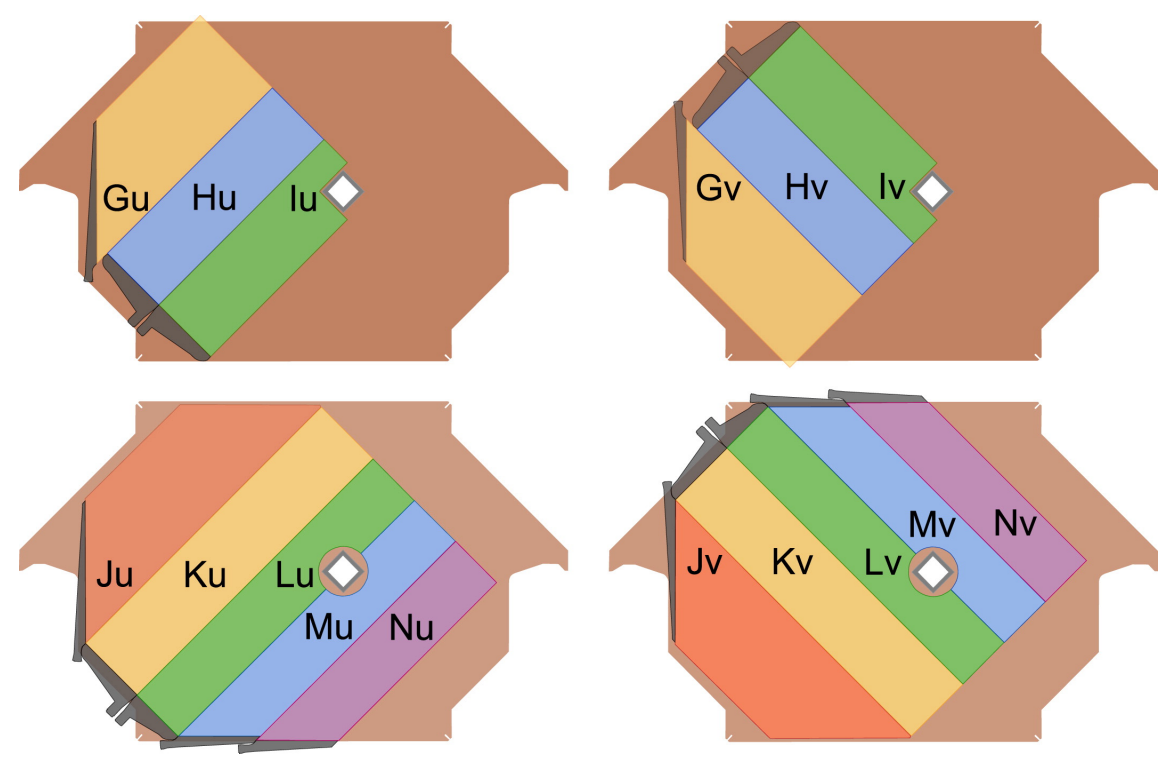

Figure 3.10: The arrangement of scintillator strips on the Near Detector planes.
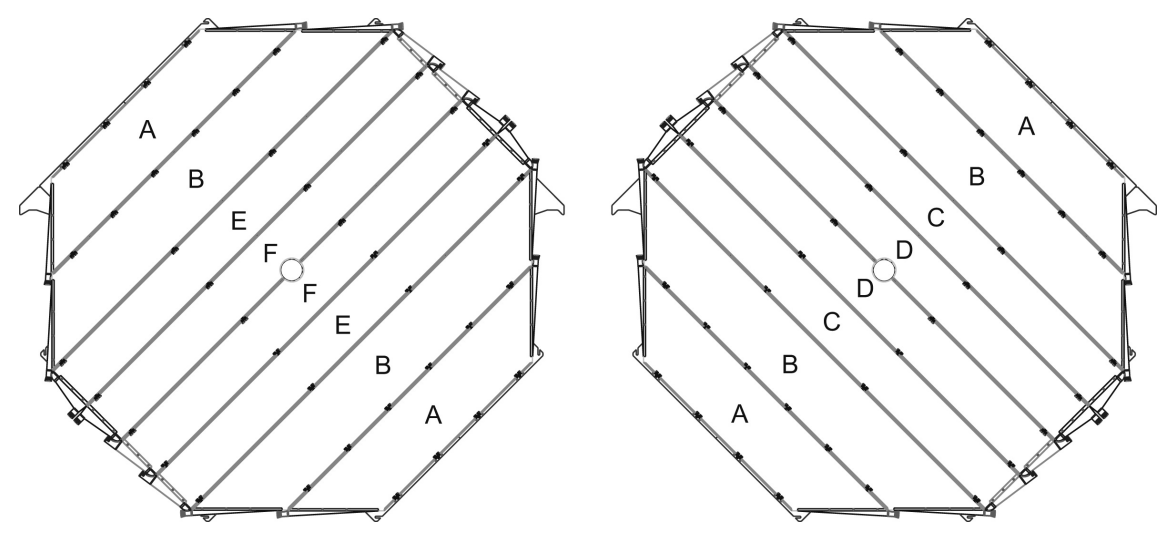

Figure 3.11: The arrangement of scintillator strips on the Far Detector planes.

\subsubsection{Calibration Detector}

To look at the response of the MINOS detectors to known-energy particles of various types a calibration detector, or CalDet, was installed at CERN. It was smaller in size but functionally equivalent model of the MINOS Near and Far Detectors. It consisted of 60 unmagnetised planes, each $1 \mathrm{~m}^{2}$ area and $2.50 \mathrm{~cm}$ thickness, of the same steel and scintillator structure as the two main MINOS detectors. The planes were instrumented with 24 scintillator strips per plane. To compare the differences in the Near and Far readout systems' 
responses, the strips were read out at one end by ND and at the other by FD electronics. Scintillation light was carried by WLS fibres. Fig. 3.12 shows a subsection of the calibration detector.

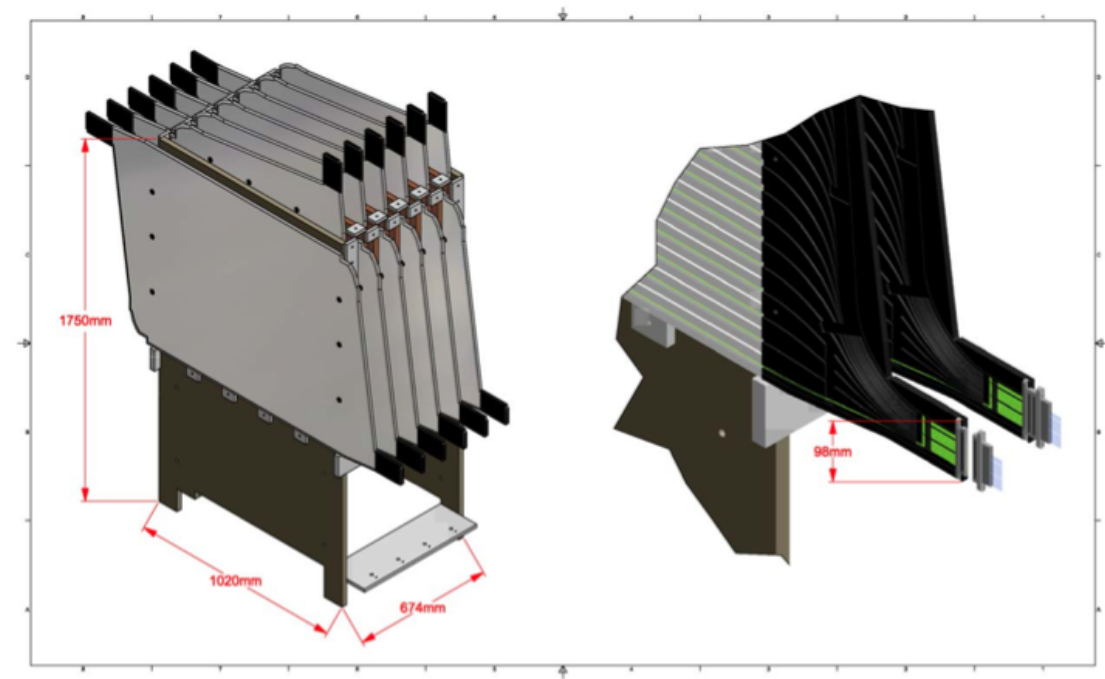

Figure 3.12: A CalDet subsection of 12 planes. The full detector was made of twelve such subsections. Detector planes are shown on the left and readout equipment on the right.

The CalDet was placed in CERN PS (proton synchrotron) test beams consisting of electrons, muons, pions and protons of momenta ranging between 0.2 and $10 \mathrm{GeV} / c$ and collected data between 2001-2003. The responses of the Near and Far Detector readout systems were found to be identical within $0.6 \%$ [104]. Observations of muons in the detector allowed a systematic error to be placed on muon energies measured from range. This will be discussed in a later section. Observations of hadronic and electromagnetic showers allowed the calorimetric energy resolution in MINOS to be measured as $(55 \% / \sqrt{\text { energy }})$ for hadron showers and $(20 \% / \sqrt{\text { energy }})$ for electrons. 


\subsection{The Detector Technology}

\subsubsection{Steel}

The average steel density is $7.85 \pm 0.03 \mathrm{~g} \mathrm{~cm}^{-3}$ [105]. The typical RMS deviation of plane masses is $0.35 \%$ (the plane thickness varying by $0.3 \%$ ) [105].

\subsubsection{Scintillator}

The structure of the scintillator strip is shown in Fig. 3.13. The strips are made of polystyrene doped with scintillator fluors PPO (1\% by weight) and POPOP (0.03\% by weight) and covered in a reflective coating of $85 \%$ polystyrene and $15 \% \mathrm{TiO}_{2}$ by weight. A charged particle travelling through the strip excites the fluor, which emits the absorbed energy in the ultra violet region peaked at $420 \mathrm{~nm}$. Wavelength shifting (WLS) fibres,

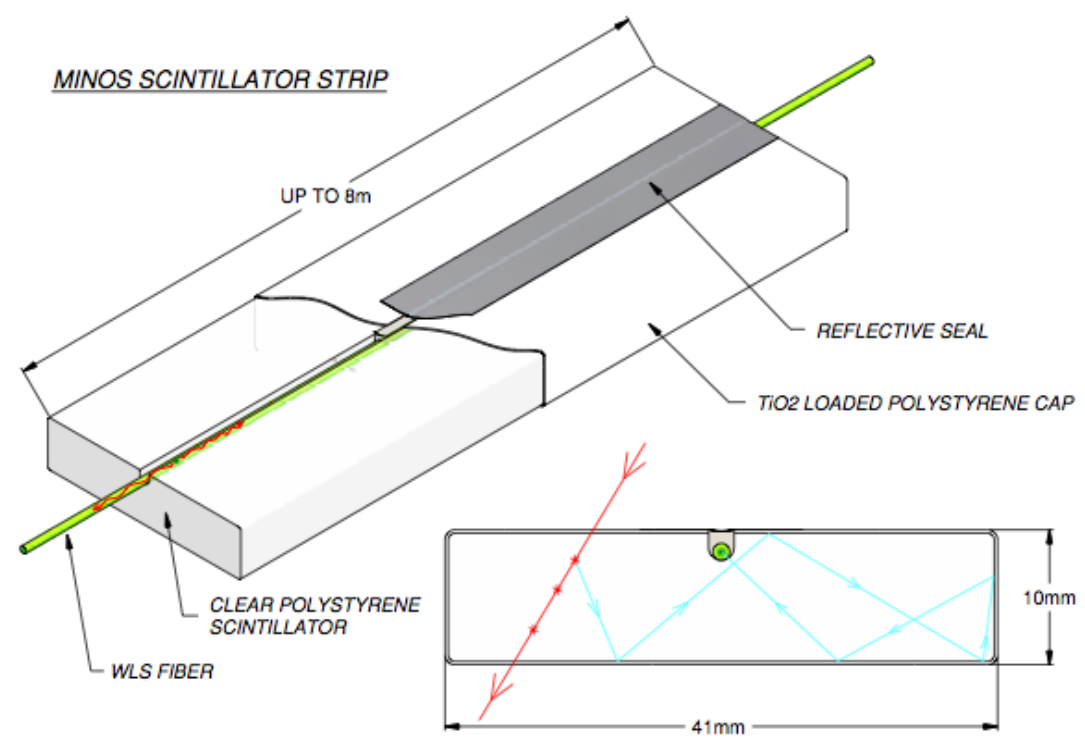

Figure 3.13: Cross-section of a MINOS scintillator strip.

$1.2 \mathrm{~mm}$ in diameter, are embedded in a $2 \mathrm{~mm}$ deep groove in the middle of each strip and run along the length of the strip. These WLS fibres absorb the UV scintillation light and re-emit it at a wavelength beyond $470 \mathrm{~nm}$, and transport it to the end of the strip. The 
scintillator strips are grouped into modules of 13 to 28 strips and enclosed in a $0.5 \mathrm{~cm}$ thick aluminium casing. The Near and Far Detector module arrangements are shown in Fig. 3.10 and 3.11 .

\subsubsection{Photomultiplier Tubes}

Light from the scintillator strips is carried by the WLS fibres, via clear fibres, to Hamamatsu multi-anode photomultiplier tubes (PMTs). The ND is read out by 64-anode (M64) PMTs each anode of which acts as a single-anode phototube; the FD by 16-anode PMTs (M16). The ND PMTs have a typical gain of $\sim 0.8 \times 10^{6}$ and the FD PMTs have gains up to $1 \times 10^{6}$. The voltage across the PMTs required to achieve these gains is $\sim 800 \mathrm{~V}$. The WLS fibres which are bundled into an optical connector, are fed to an optical cable. This cable carries the signal into the multiplexing (MUX) box. Inside the box, clear fibres carry the light onto the face of the PMT. Fig. 3.14 shows the readout of scintillator strips by photomultiplier tubes. The PMTs are housed in steel crates, which are light-tight and also shield the PMTs from the magnetic field.

In the FD, eight scintillator strips from a single detector plane, which are at least $1 \mathrm{~m}$ apart, are read out by each PMT anode. This is feasible because the transverse spread of hadronic or electromagnetic showers from beam neutrinos is limited within $1 \mathrm{~m}$ region. To enable determination of which strip was actually hit, the 8 strips read out by a single pixel on one side of the detector are read out by 8 different pixels on the other side. The FD gains are linear up to 100 photoelectrons and have a quantum efficiency (QE) greater than $12 \%$ for a photon of $520 \mathrm{~nm}$ wavelength.

Each scintillator strip in the first 120 planes of the ND is read out individually by one anode. In the remaining planes there is a 4:1 multiplexing, where four scintillator strips from a single plane are read out by a single anode. The four multiplexed strips are separated by approximately $1 \mathrm{~m}$. This allows the ambiguities in muon tracking to be resolved during event reconstruction using information from adjacent planes to identify the actual strip hit. 


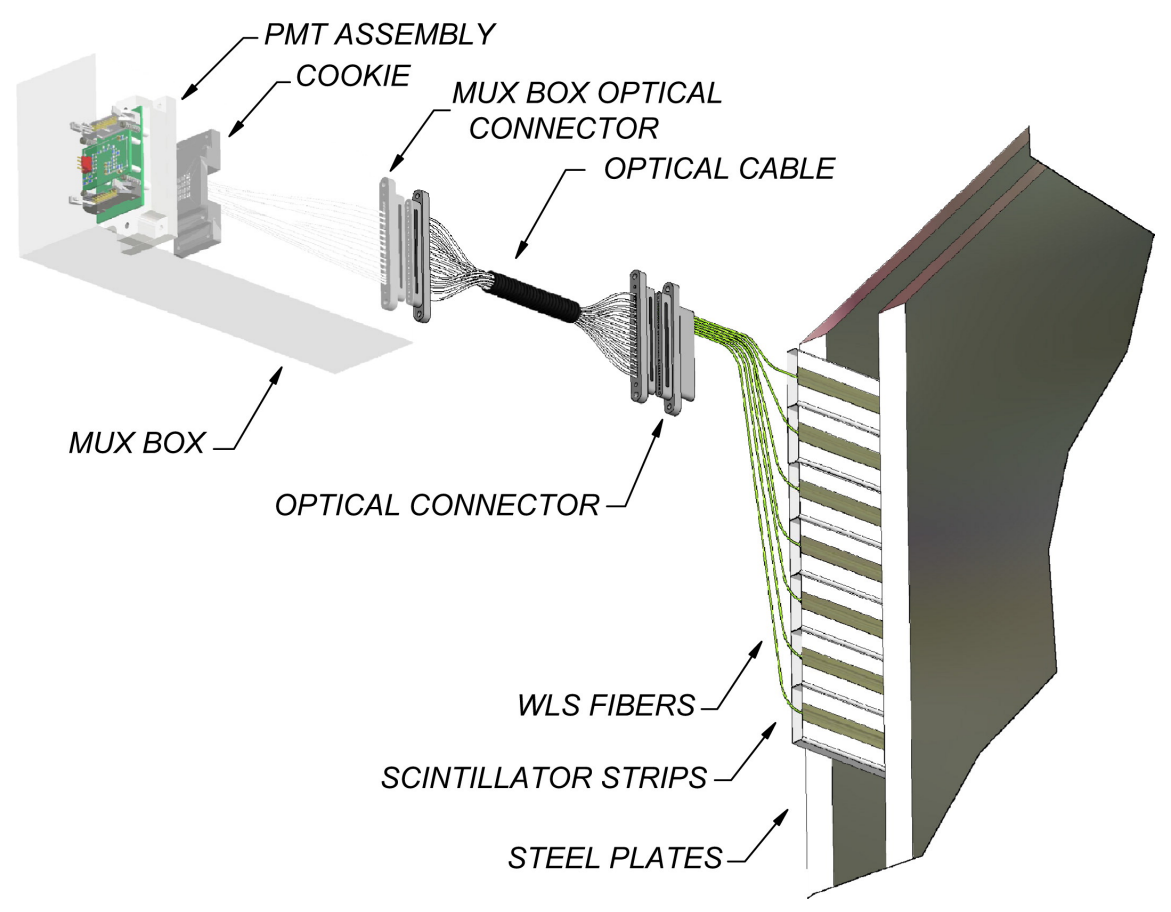

Figure 3.14: Schematic drawing of the scintillator system readout for a module. An edge of a detector plane is on the right side of the sketch, showing several strips extending out of a scintillator module and beyond the edge of the plane for clarity. The light produced in a strip travels out of the module in a WLS fiber, and is then carried by a clear optical fiber (assembled into a cable) to a MUX box where it is routed to a pixel of the PMT assembly.

The PMTs have a linear response for input pulses of up to 50 photoelectrons. The quantum efficiency (QE) is about $12 \%$ for a photon of $520 \mathrm{~nm}$ wavelength.

An artifact of the multi-anode design of the PMTs is that charge can drift from one anode to another. This allows a low pulse height hit (usually of less than one photoelectron) to be registered at a nearest neighbor of an anode. This phenomenon is referred to as cross talk. Total cross talk in all pixels of PMTs is $3.74 \%$ in the ND and $3.2 \%$ in the FD PMTs.

\subsection{The Magnetic Field}

Both MINOS detectors are magnetised by a coil of current-carrying cables that passes through a hole cut through the length of the detector. The FD coil hole is at the centre of the octagon and each supermodule is magnetised independently by its own coil. The coil consists of 190 turns of copper wire with Teflon insulation and is enclosed in a water-cooled 
copper jacket. Fig. 3.15 shows the cross section of a FD coil. An 80 A power supply gives a $15.2 \mathrm{kA}$-turn total current providing an average toroidal magnetic field of $1.3 \mathrm{~T}$ strength [105]. One coil dissipates about $20 \mathrm{~kW}$ of power.

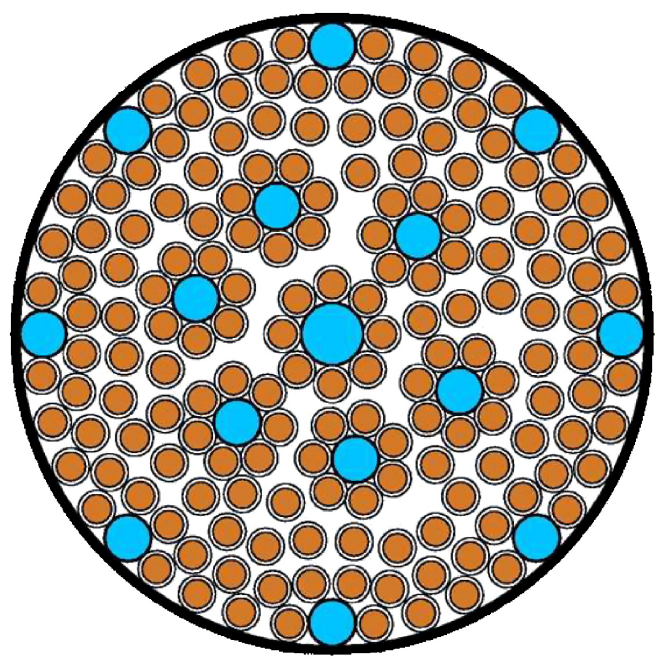

Figure 3.15: Cross-sectional view of one of the Far Detector supermodule coils. The larger diameter circles represent the copper cooling tubes and the smaller circles are the 190 turns of $1 / 0$ gauge stranded copper wire.

The ND coil hole is offset $55.8 \mathrm{~cm}$ from the centre of the plane and the detector is placed such that the beam is centred half-way between the hole and the left vertical edge of the detector. This minimises the number of tracks that travel through the uninstrumented coil hole region and get reconstructed poorly. Due to the squashed octagon geometry, a $40 \mathrm{kA}$-turn current is required to achieve sufficient field [105]. The cooling is provided by a closed loop low-conductivity water (LCW) system that transfers out the heat from the cables. The coil has eight turns and dissipates a power of $\sim 47 \mathrm{~kW}$. The magnetic field in both detectors' fiducial volumes are nearly uniform. Field maps of both detectors can be seen in Fig. 3.16 .

\subsection{Electronics and Data Acquisition}

While the Near and Far Detectors were designed to be as similar as possible to minimise the effect of systematic uncertainties, the same electronics could not be used for both the 


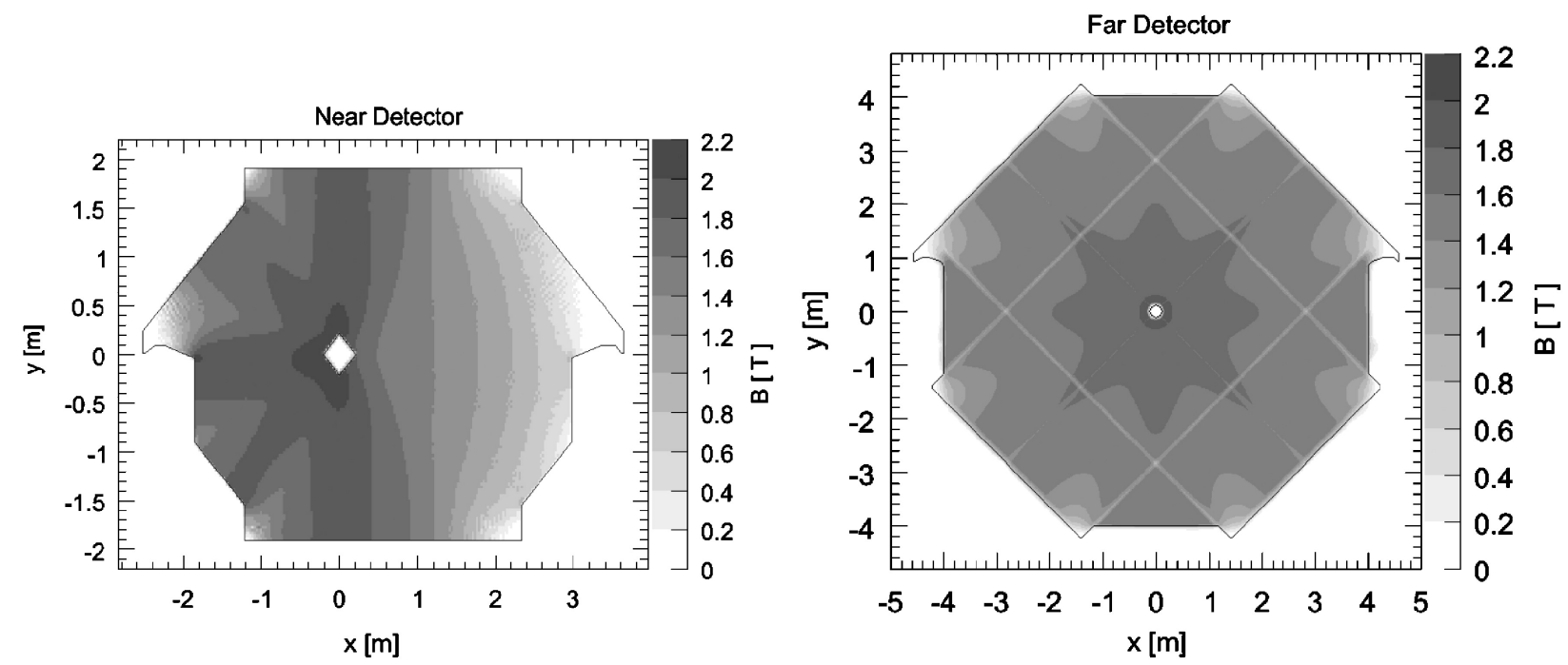

Figure 3.16: Magnetic field maps for a typical near (left) and far (right) detector planes. The grayscale indicates the magnetic field strength B as calculated by finite element analyses using 3-D models. The detector planes are shown looking upstream to the neutrino beam.

detectors. Since the FD is $705 \mathrm{~m}$ underground, the cosmic muon rate is around $0.5 \mathrm{~Hz}$. Furthermore, the beam neutrino interaction rate is of the order of a few per day. By contrast, in the ND there are of the order of 10 neutrino interaction events per $8-10 \mu$ s long spill. Due to the widely different neutrino event rates and cosmic ray interaction rates, the two detectors have different electronic requirements. The primary goals of the electronics are to provide adequate information for the separation of neutral and charged-current neutrino events and to enable the measurement of the energies of particles with minimum bias. The design goals of the data and acquisition systems (DAQ) are described below. The Near and Far Detector front end electronics are described separately, followed by a section describing the DAQ which is common to both the detectors.

\subsubsection{Near Detector Front-End Electronics}

In order to reconstruct all the events produced, the ND electronics are designed to be fast and have no deadtime [105, 106]. The shortest time required to be resolved is that between adjacent buckets of MI protons: the smallest discrete groups of protons forming the neutrino 
beam. The size of these buckets is defined by the frequency of the accelerator's RF cavities: $53 \mathrm{MHz}$. The ND electronics is therefore designed to have a time resolution of $18.8 \mathrm{~ns}$.

The signal from each PMT anode is fed into a QIE (Charge (Q) Current (I) Encoder), which consists of a current splitter, gated integrator and range selector. The signal first encounters a current splitter, which divides the current into eight ranges with values I/2, $\mathrm{I} / 4, \ldots, \mathrm{I} / 256$ and integrated onto a capacitor for each range. See Fig. 3.17 for a plot of the QIE response. Each capacitor is sensitive to its respective range of current. The capacitors of all ranges below that corresponding to the signal size are saturated, the current in the first unsaturated range providing the precision measurement of the signal. A bias current is added to ensure that the voltage across one and only one of the capacitors is within the predetermined limits of the ADC, which is selected by the range selector and output to the ADC, along with a three bit number indicating the range value. This system works to increase the dynamic range of the readout.

The current splitter integrates all the current in a single $53 \mathrm{MHz}$ clock cycle, the first unsaturated range being selected in the next cycle. In the subsequent cycle this analog signal is converted to an eight bit digital signal using an ADC (analog to digital converter; not part of the QIE itself). A fourth clock cycle is required to reset the capacitors in the current splitter. To prevent the readout being dead for the three clock cycles following the integration stage four current splitters reside on each QIE, each integrating in turn for one cycle, all using the same range selector and ADC. In addition to the 8-bit ADC value and the 3-bit range value, a 2-bit code (called CAPID) identifies the current splitter and capacitor from which the signal was read. This 13-bit word is received by a 1000 word FIFO (first in, first out) buffer. This FIFO is large enough to buffer all words in a $10 \mu$ s spill.

The QIE is mounted on a board called MENU (MINOS Electronics for Neutrinos), the smallest front-end electronics unit. A MENU holds a QIE chip, an ADC and a FIFO along with a DC current injector which can inject a signal into the QIE for calibration as shown in Fig. 3.18 , 


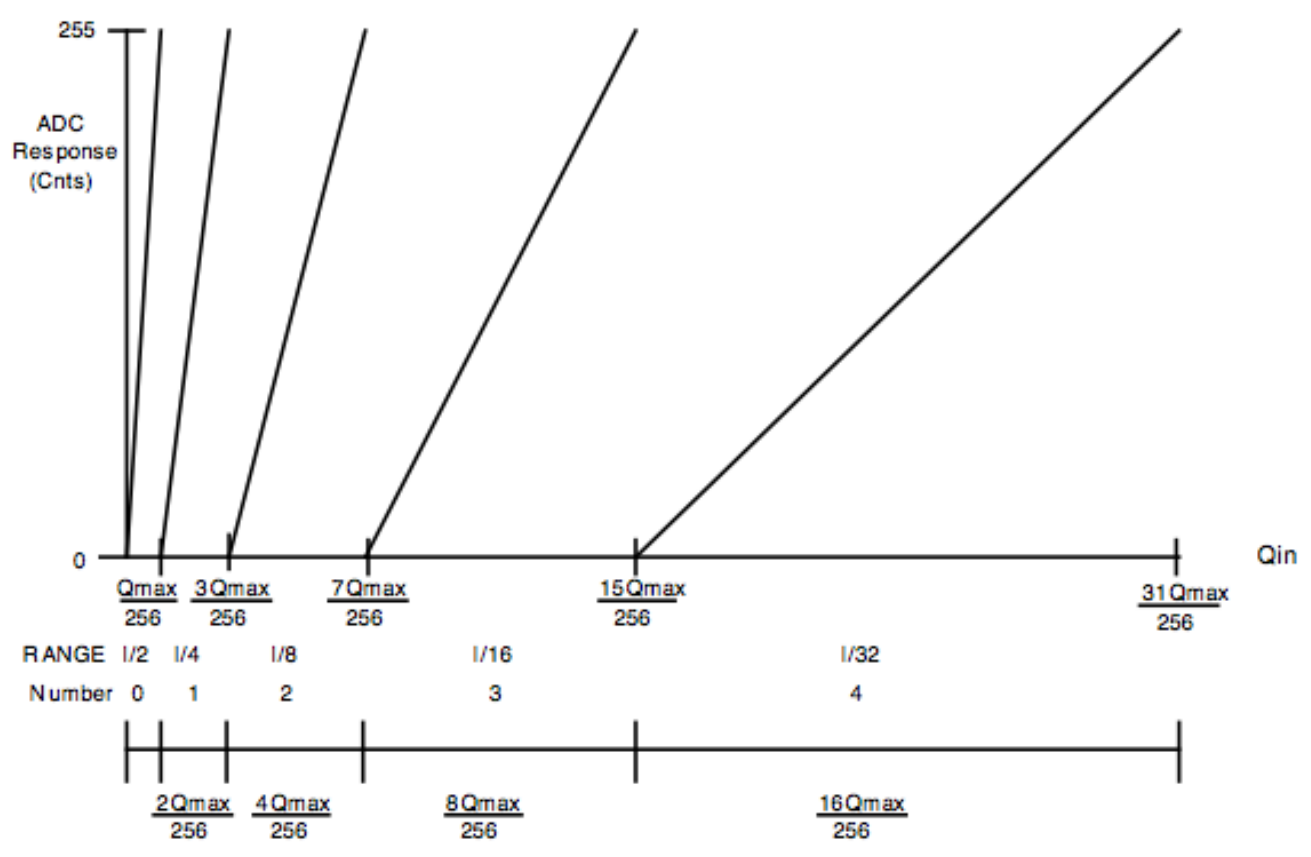

Figure 3.17: Response of the QIE-chip based electronics: x-axis shows the amount of charge injected into the chip, while y-axis shows the ADC response for the different ranges. Only the first five out of the eight ranges are shown.

Sixteen MENU modules are mounted on a MINDER module (MINOS ND Electronics Readout), which time-stamps the signals and controls the modes of the MENUs (e.g. initiating the calibration mode). The MINDER reads sequentially all words from all its FIFOs, sending them to a single FIFO on the next module in the chain: the MASTER module (MINOS Acquisition, Sparsifier and Timestamper for Event Readout). Each MASTER module holds eight MINDERs. It reads the data from these MINDERs into two buffers, which are read out by a VME computer (one buffer is written to while the other is being read). The VME computers send the data to the data acquisition (DAQ) system. Fig. 3.19 shows the schematic overview of the ND front end electronics.

\subsubsection{Far Detector Front-End Electronics}

The FD electronics were specifically designed for the low rate underground environment, as discussed in [107]. The neutrino beam generates only a few events per day and the cosmic 


\section{MENU Module}

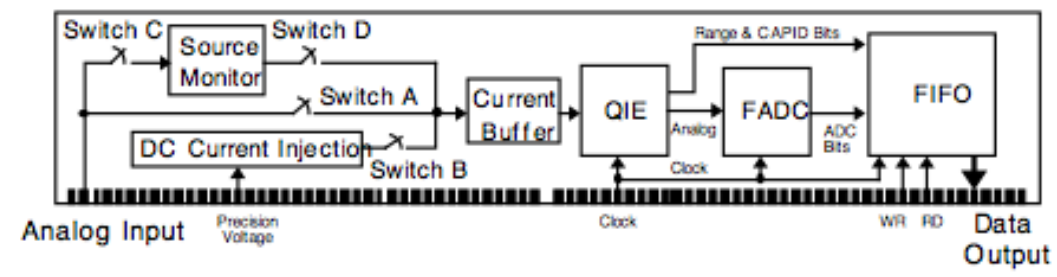

MINDER Module

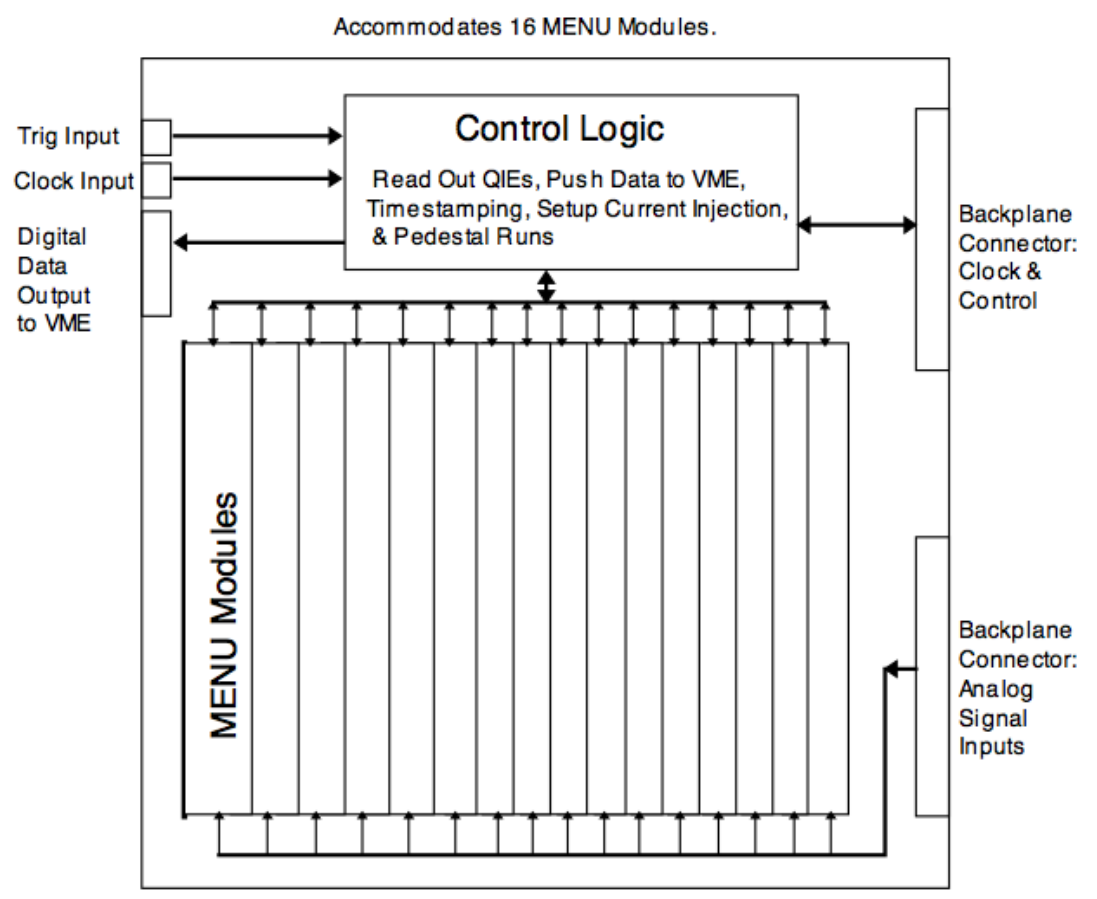

Figure 3.18: Block diagram of a MENU module and a MINDER module.

muon rate $705 \mathrm{~m}$ underground is $\sim 0.5 \mathrm{~Hz}$. Therefore some deadtime can be tolerated. A diagram of the FD front-end electronics is presented in fig. 3.20 .

Each PMT is read out by a single chip, called a VA chip. Only 17 of its 32 channels are used. 16 channels are used to read out an anode each, and one channel reads out the PIN diode monitoring the light injection system, see Sec. 3.1. The VA chips are mounted on VA 


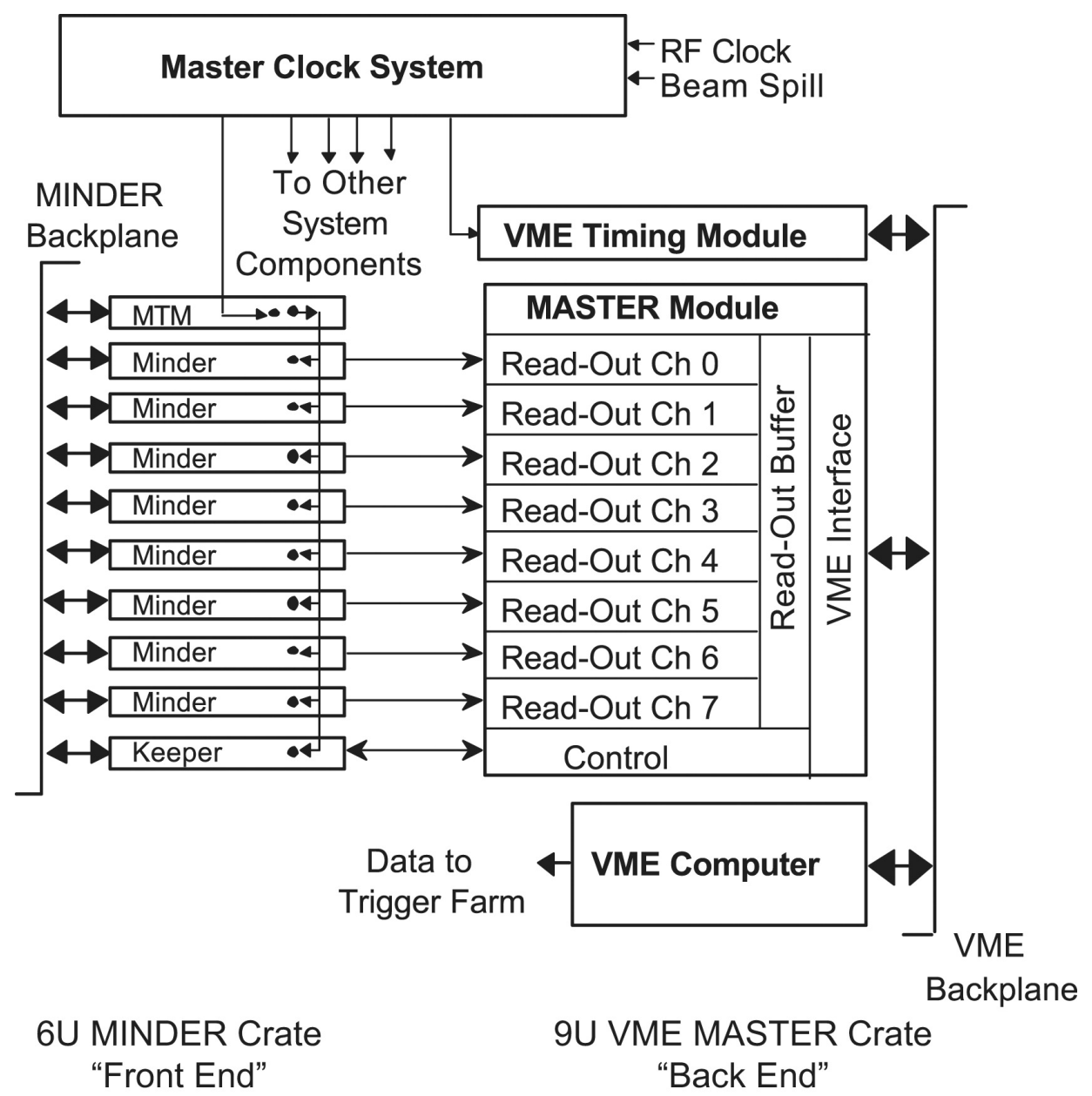

Figure 3.19: Overview of the MINOS Near Detector readout electronics.

front-end boards (VFB) which provide power control and biasing for the VA chips. The VFB also houses a chip called ADSLite. This chip compares the dynode signals from the PMTs with a common programmable threshold to provide a discriminated signal for time-stamping and readout initiation.

The VFBs feed an analog/digital converter which is housed on a VMM (VA Mezzanine Module); two VFBs are connected to one VMM. The VARC (VA Readout Controller) handles signal digitization, triggering, time-stamping and bias of the VA chips. The VFBs are fully controlled by the VARC. One VARC module reads 36 PMTs through 6 VMMs, each of which read 6 PMTs. The digitization of the VA chip takes $5 \mu \mathrm{s}$, meaning if all six need to be read out, all six chips would be dead to new signals for $\sim 30 \mu$ s. In order to alleviate 
the deadtime cost of reading out, at least two dynode signals within a 400 ns window are required to trigger readout.

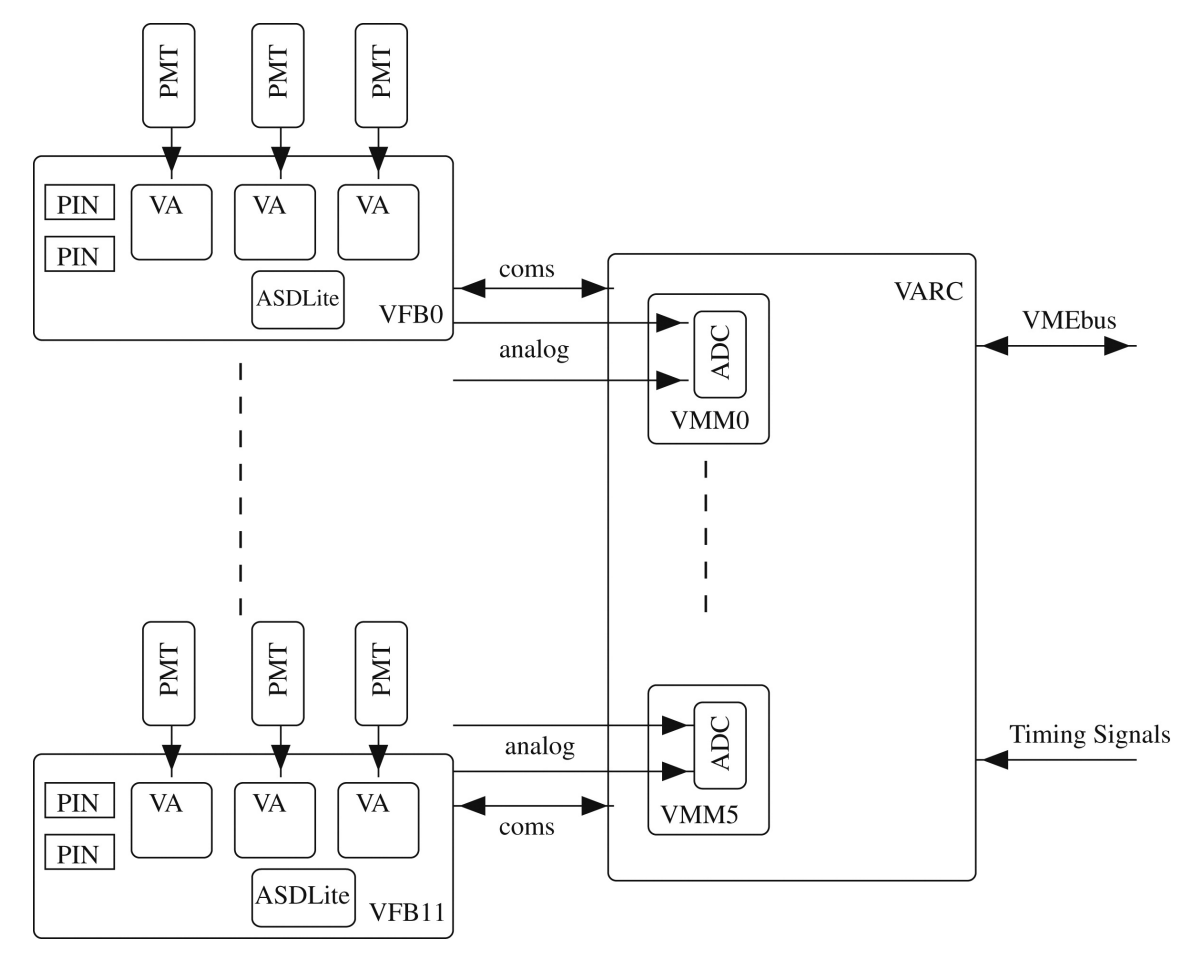

Figure 3.20: Overview of the MINOS Far Detector readout electronics.

The digitized data are sent to a FIFO, pedestal subtracted then written to an on-board VME memory, which is subsequently read out by the DAQ.

\subsubsection{Data Acquisition}

The Near and Far Detector data acquisition systems are functionally identical, with appropriate front-end software accommodating the differences of the front-end electronics of the two detectors. A diagram of the FD DAQ components is shown in Fig. 3.21. The DAQ system is designed to continuously read out the front-end electronics in an untriggered, dead time free manner and to transfer the data from all front-end modules to a small farm of PCs where software algorithms build and select events of interest and perform monitoring and calibration tasks. A detailed description of the DAQ system architecture can be found in [108]. 


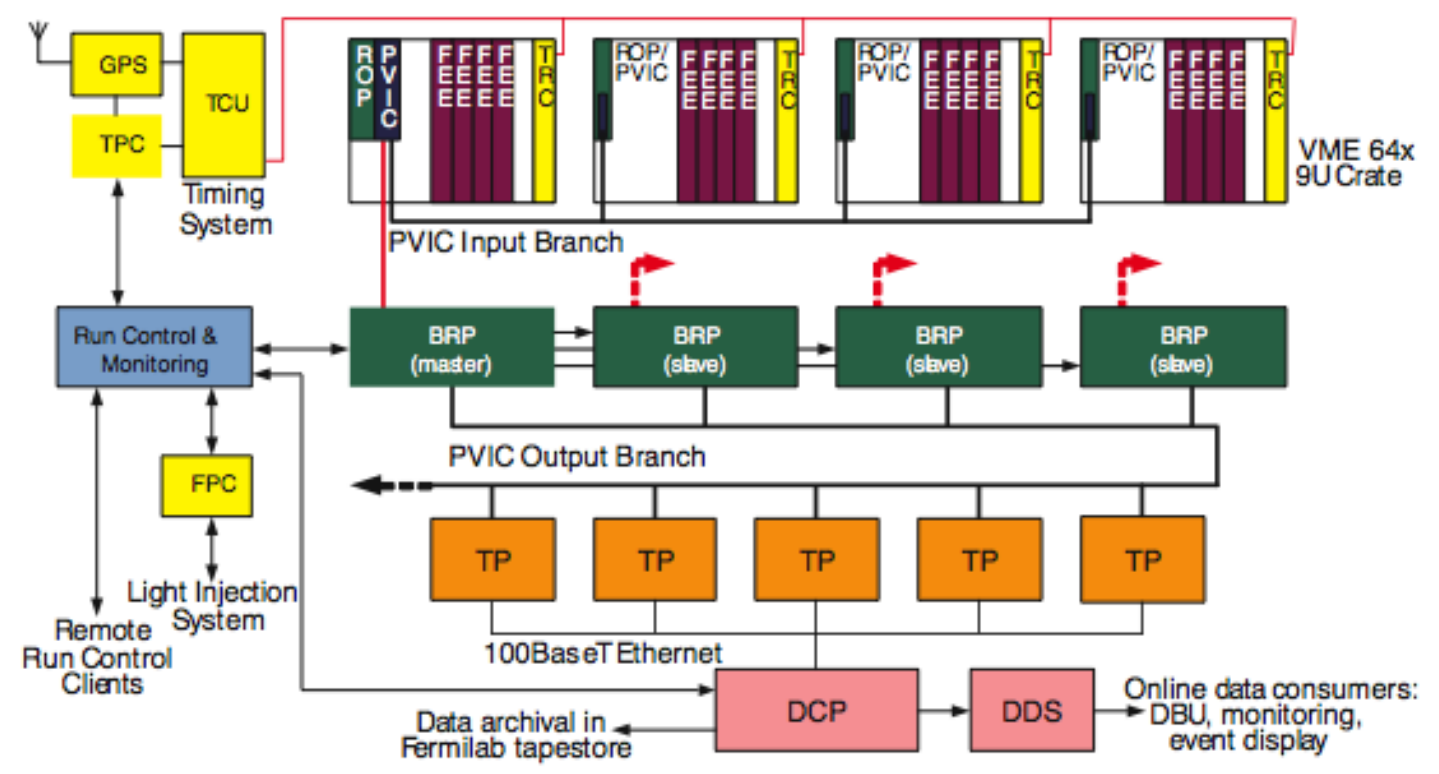

Figure 3.21: The architecture of the MINOS DAQ system for the Far Detector. For clarity, only one of the six PVIC input branches is completely illustrated.

The DAQ reads out data continuously but only writes it to disk under certain conditions. The ND DAQ is triggered by the beam spill signal at Fermilab and all data arriving within a $100 \mu \mathrm{s}$ window around the spill is recorded. Since the FD is not at Fermilab, it cannot be triggered directly by the beam spill. Instead, it receives the GPS timestamp of the beam spill from the ND via the Internet.

The MASTER module described earlier has two buffers, one of which is being written while the other is being read out by a Readout Processor (ROP). There are 16 ROPs serving the FD and 8 serving the ND. The buffers are read for the duration of one timeblock, 10-50 ms long. Each ROP assembles consecutive time blocks in memory for one time frame, which is nominally one second in duration. When requested, the ROPs transfer them to Branch Readout Processors (BRPs). One BRP acts as the master and coordinates the transfer of data. Each BRP is also connected to an output branch which connects the BRPs to a small farm of trigger processors (the TPs). When a time frame has been successfully transferred to the BRPs, the master BRP selects a TP and instructs each BRP to send its time frame to an address in the TP memory. This way a full time frame of data from the entire detector 
is available in a single TP for processing.

The TP performs a number of processing tasks on the time frame data, and also applies software triggering algorithms to locate events of physics interest. The output from the trigger is called a snarl and, in principle, can gather multiple events (notably the spill triggers). The primary triggers are summarised below. The triggers fall into three categories: special triggers for debugging and calibration, bias-free triggers based on spill signals or spill times to gather beam events, and triggers based on the clustering of hits in the detector to gather out-of-spill events.

(i) Spill trigger: At the ND, each digitization that occurs within the spill gate is tagged by the front-end electronics. These are identified, extracted from time frame and output as a single spill event with no further selection.

(ii) Remote spill trigger: At the FD a direct spill signal is not available so a remote spill trigger is applied. The ND GPS system is used to generate time-stamps of the spill signals. These are transmitted to the FD over the internet where they are stored and served to TPs on request. All readout within a configurable time window around each spill is extracted and written out as a spill event.

(iii) Fake remote spill trigger: In addition to the actual spill trigger, fake spill times are also generated randomly between spills to provide random sampling of detector activity.

(iv) Plane trigger: This is a customisable trigger used to write out events when $M$ detector planes in any set of $\mathrm{N}$ contiguous planes contain at least one hit. Nominally $M=4$, $N=5$ for physics analysis.

(v) Energy trigger: This trigger is used to write out events when $M$ contiguous planes of the detector have a summed raw pulse height greater than $E$ and a total of at least $N$ hits in those planes. Nominally $M=4, E=1500$ ADC counts and $N=6$.

(vi) Activity trigger: This requires $N$ planes to register activity across the detector. Nominally $N=20$.

(vii) Special triggers: In addition to the above triggers, a number of special triggers are 
available which are used when performing detector and electronics calibration or debugging. 


\section{Chapter 4}

\section{Calibration, Event Reconstruction and Monte-Carlo Simulation}

\subsection{Calibration}

The oscillation analyses in MINOS rely on the comparison of neutrino energy spectra and event characteristics in the near and far detectors. The measurement of neutrino energy further depends on the accurate measurement of the absolute muon energy and absolute hadronic energy deposited in the detector after a neutrino interaction. The detector response can vary within the detector region and also with time which may significantly affect the accuracy of this measurement. A calibration system is used to account for these variations and to make the energy response of the near and far detectors to be consistent with each other.

MINOS uses a multi-step calibration chain to convert the raw detector pulse height to a fully corrected signal by correcting for the scintillator light output variations as well as nonuniformities of light transmission and collection in fibres, PMTs and readout electronics. The calibration uses both the optical light-injection (LI) system, which measures the behaviour of the readout instrumentation, and cosmic ray muon tracks, which give the response of the scintillator. The full chain can be broken down as follows: 


$$
\begin{aligned}
Q_{\text {corr }}= & Q_{\text {raw }}(s, x, t, d) \times D_{\mathrm{PMT}}(d, t) \times L\left(d, s, Q_{\text {raw }}\right) \\
& \times D_{\text {scint }}(d, t) \times S(s, d, t) \times A(d, s, x) \times M(d)
\end{aligned}
$$

where $s$ is the strip hit, $x$ is the position in the detector, $t$ is the time of the hit, $d$ is the detector (near or far) where the hit occurred. $Q_{\text {raw }}$ is the raw photomultiplier response; $D$ is the drift correction that accounts for changes in scintillator, PMT and electronics response with temperature and time; $L$ linearises the response of each channel; $S$ removes differences from strip to strip; $A$ corrects for attenuation depending on the position of the hit in the strip, and $M$ is the energy scale factor that corrects the pulse height to the same energy unit in both detectors.

\subsubsection{Calibration Procedure}

\section{PMT and Electronics Drift, $D_{\mathbf{P M T}}(d, t)$ :}

The light injection (LI) system [109, 110] is designed to monitor and compensate for changes in the PMT gains and non-linearity. It uses light-emitting diodes (LEDs) to inject ultraviolet light into the wavelength shifting fibres, simulating scintillator light. A set of 20 LEDs are housed in a pulser box and illuminate multiple individual fibres. The intensity of injected light is monitored by PIN diodes while being simultaneously read out by PMTs. The LI system pulses periodically at the end of every strip: 300 times per hour at the FD and 1000 times per hour at the ND. This is designed to average 50 photoelectrons per pulse per PMT pixel in both detectors. Changes in LED intensity can lead to variations of up to a factor of two in this light level; such changes are monitored and corrected.

This LI data is collected once a month at each detector. The PMT gains are measured using photon statistics [109]. Gains measured by the LI system provide corrections to the PMT gain and electronics response. Short term variations that are usually due to tempera- 
ture variations can be eliminated by good environmental control, while longer term variations due to aging and changes in hardware amount to $\sim 4 \% /$ year.

Linearity, $L\left(d, s, Q_{\text {raw }}\right)$ :

The PMT and electronics responses are not linear for light levels greater than approximately 100 photoelectrons. The LI system is utilised to correct for this nonlinearity by injecting a known intensity of light into scintillator strips, ranging from a few to hundreds of photoelectrons, and measuring the electronics response as a function of true illumination. In the ND, the charge integration and ADC electronics are also calibrated for linearity by directly injecting charge into the front-end electronics.

Scintillator Drift Correction, $D_{\text {scint }}(d, t)$ :

The scintillator response changes in time as the material ages. Aging reduces the light level by about $2 \%$ annually and environmental variations, mainly temperature, result in short-term changes. The response of the detectors is measured by monitoring the median response of a detector plane to through-going cosmic muons. Although the spectra of cosmic muons are different between detectors, due to differences in depth, zenith angle and latitude, the energy spectra and rate of cosmic muons going through one detector is time independent. It is therefore possible to calibrate for this drift by comparing the median detector response at time $t$ to the response at time $t_{0}$, the beginning of the experiment:

$$
D_{\text {scint }}(d, t)=\frac{\text { Median response }\left(d, t_{0}\right)}{\text { Median response }(d, t):}
$$

Strip-to-strip Calibration, $S(s, d, t)$ :

The strip-to-strip non-uniformity correction accounts for several detector effects including scintillator light yield, WLS collection efficiency, differences in attenuation in the optical fibres, PMT quantum efficiency and PMT gain. The correction is obtained by using cosmic ray muons in the minimum ionising energy range (a few GeV) [105], since these muons' 
energy deposition is relatively independent of their momenta. An individual strip's response is characterised by constructing the muon spectrum for that strip from cosmic ray muon data accumulated over a period of time. The calibration constant is calculated every month as the strip response normalised by the mean response of all strips:

$$
S(s, d, t)=\frac{\text { Mean response of whole detector }(d, t)}{\text { Response of } \operatorname{strip}(s, d, t):}
$$

Attenuation, $A(d, s, x)$ :

This correction accounts for the variation in light as a function of distance along the strip mainly because of attenuation in the WLS fibres. The calibration constant is given by:

$$
A(d, s, x)=A_{1}^{d, s} e^{-x / L_{1}^{d, s}}+A_{2}^{d, s} e^{-x / L_{2}^{d, s}}
$$

where $x$ is the transverse position along the strip and $L_{1}^{d, s}$ and $L_{2}^{d, s}$ are the attenuation lengths. This correction was calculated by using a module mapper. The module mapper measured the response of each scintillator module to ionising radiation using a ${ }^{137} \mathrm{Cs}$ source. To obtain the calibration constant, i.e., the values of $A_{1}, A_{2}, L_{1}$ and $L_{2}$, a fit is performed for each strip and the resulting parameters are used to correct the data [105]. The attenuation correction was later cross checked by using through-going muons and was found to be consistent to within $4 \%$.

Inter-detector Calibration, $M(d)$ :

After correcting each of the three detectors' responses to be spatially and temporally consistent within one detector, the calorimetric responses of the near, far detectors and CalDet are calibrated to normalise the energy scales of the three detectors. This calibration is performed by using stopping cosmic ray muons. Stopping muons are used because their momenta can be accurately determined from their range. The energy loss of a muon by ionisation, as described by the Bethe-Bloch equation, is shown in Fig. 4.1. 
All the MINOS detectors have the same scintillator strip width. Therefore, by measuring the detectors' response to muons of a certain track length the inter-detector calibration can be performed. A "track window" technique is used to maximise the accuracy, where the response of muons is only measured when their momenta lie between 0.5 and $1.1 \mathrm{GeV}$. This avoids using the end of the track where the losses by ionisation increase very rapidly (as shown in the left side of the curve of Fig. 4.1). This minimises the dependence on the muons' momentum measurement. This technique allows for the determination of the interdetector energy scale with a $2 \%$ uncertainty. The scale is measured in terms of Muon Energy Units (MEUs). One MEU corresponds approximately to the average detector response to a minimum ionising muon traversing 1 plane of scintillator at normal incidence.

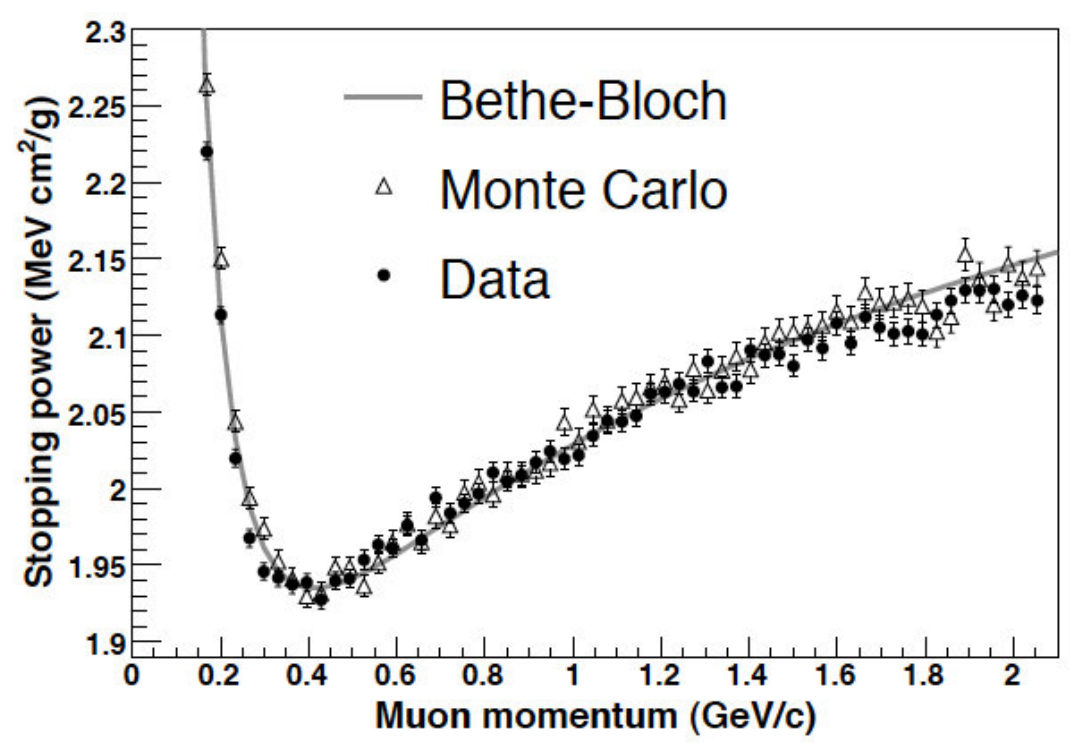

Figure 4.1: Stopping power of muons as a function of their momentum. The prediction by the Bethe-Bloch equation in polystyrene scintillator agrees very well with the MINOS FD data and the MINOS MC simulation. Both data and MC points have been normalised to the Bethe-Bloch calculation to give the expected stopping power at the minimum ionising point. Image from [105]. 


\subsubsection{Absolute Energy Scale}

As a final step the energy depositions in the near and far detectors are expressed in terms of an absolute energy scale. This way the response of the detectors can be understood for muons, electrons and hadrons of a certain energy.

The absolute track and shower energies were determined using data collected by CalDet while it was exposed to various test beams of known composition and energy at CERN. The CalDet collected data with pions, protons, electrons and muons of both positive and negative charge signs in the energy range $200 \mathrm{MeV}$ to $10 \mathrm{GeV}$ with both near and far detector electronics. By comparing the calibrated shower energy (in MEUs) to the known energies of the particle beams (in $\mathrm{GeV}$ ) the absolute calibration of the detector energy response is achieved. The CalDet data were also used to benchmark the hadronic and electromagnetic shower simulation and to determine the energy resolution. Fig. 4.2 shows the measured detector response to pions and electrons compared to the simulation result. The range of stopping muons (of momenta smaller than $2.2 \mathrm{GeV} / \mathrm{c}$ ) was modeled to better than $3 \%$ accuracy with GEANT3, while the detector response to electrons was simulated with an accuracy better than $2 \%$. The pion and proton induced showers were reproduced to better than $6 \%$ accuracy with the GCALOR [111] simulation. The energy resolution was adequately reproduced by the simulation and may be parameterised as $56 \% / \sqrt{E} \bigoplus 2 \%$ for hadron showers and $21.4 \% / \sqrt{E} \bigoplus 4 \%$ for electromagnetic showers, where the energy $\mathrm{E}$ is in $\mathrm{GeV}$.

A summary flow chart of the calibration procedure is given in Fig. 4.3. The plots in Figures 4.4 and 4.5 show how the calibration chain affects the response in the far and near detectors, respectively. It is clear that the response is very uniform across the whole detector after calibration. But the calibration procedure is not perfect, and differences in calorimetric response between the two detectors to the same energy scale are incorporated as detector-specific systematic uncertainties.

Recent studies have checked the consistency of the MINOS calibration procedure by 


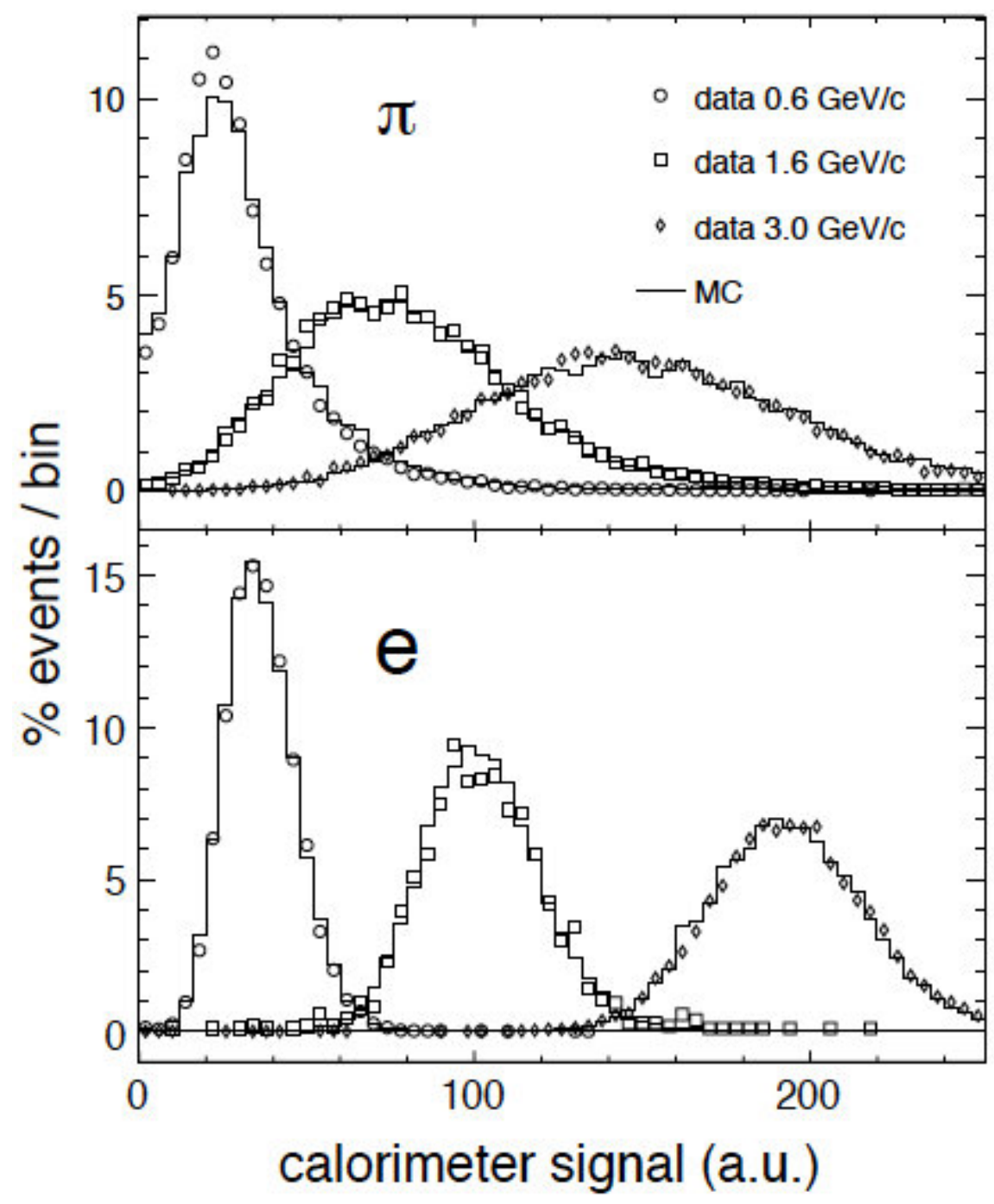

Figure 4.2: Calorimetric response to pions and electrons at three momenta. The calorimetersignal scale is in arbitrary units. The data obtained from CalDet is compared to the simulation. Image from [105.

calculating the change in drift, PMT response and light level changes in the FD and ND since the beginning of MINOS running. As shown in Fig. 4.6 the response is found to be stable within $0.5 \%$ and $1.5 \%$ for ND and FD, respectively. The light level studies performed on the FD to measure the change in the light level obtained from the PMTs since the beginning of MINOS running have shown that the detectors are aging non-uniformly. More details on the light level studies are given in Appendix A. 


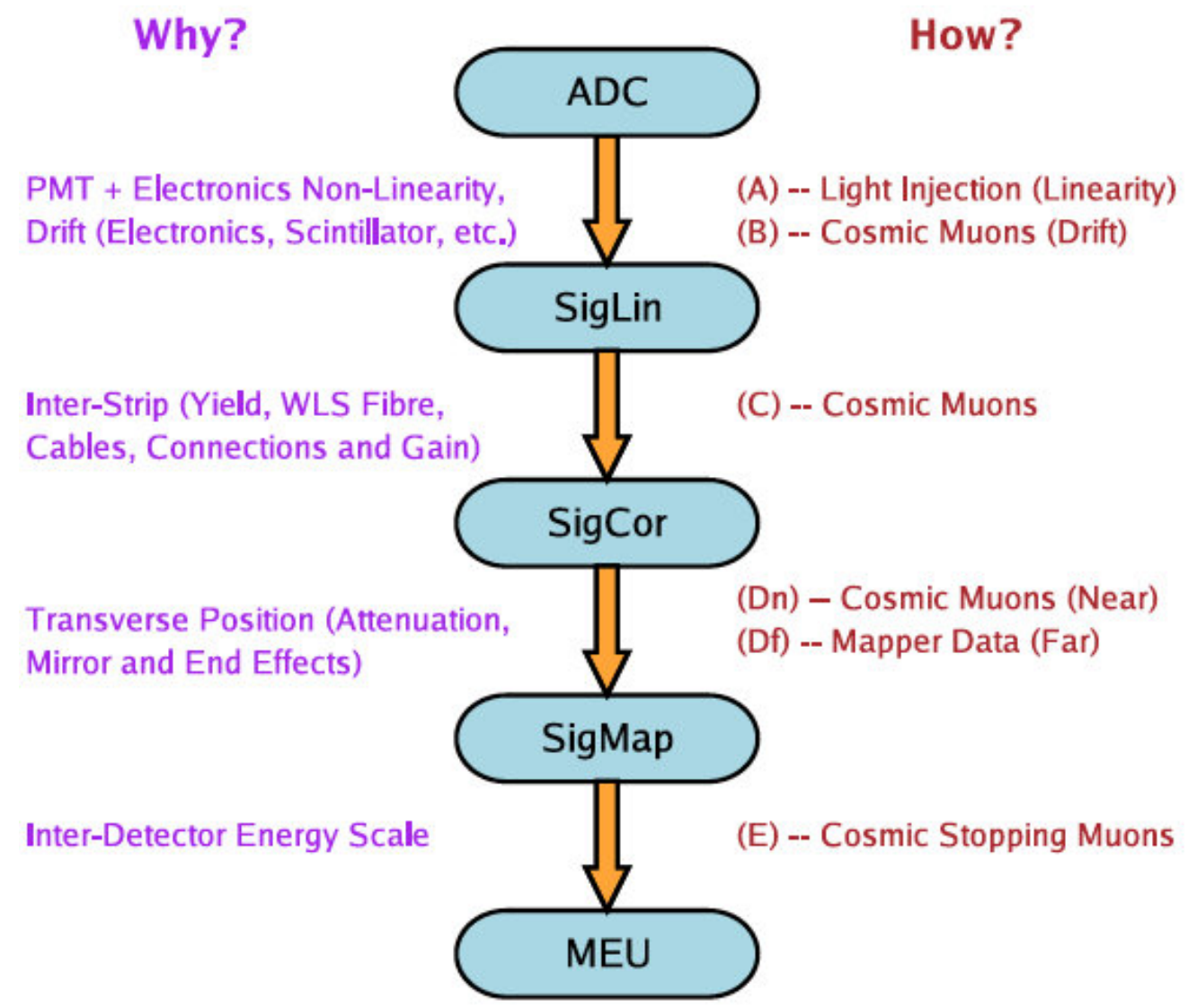

Figure 4.3: A summary of the MINOS calibration procedure. Each step corresponds to a correction factor in Equation 4.1. The correction from raw pulse height (ADC) to SigLin applies the linearity calibration, L and drift correction D; from SigLin to SigCor the stripto-strip calibration S; from SigCor to SigMap the attenuation correction A and finally the absolute energy scale calibration $M$ to convert the raw signal into the standardised energy unit MEU. Taken from [105].
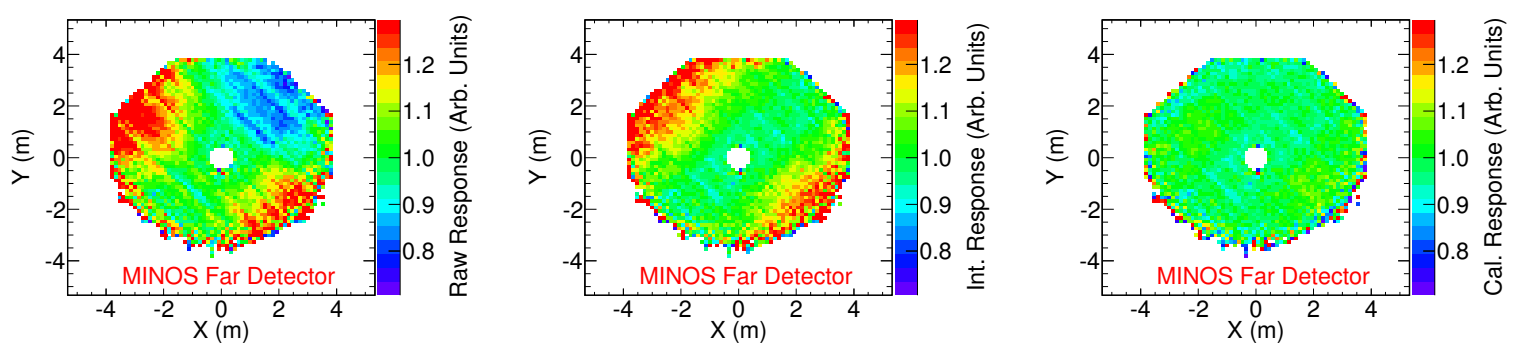

Figure 4.4: Response of the Far Detector in ADCs(left), in SigCors(middle) and in SigMaps(right), as a function of detector position, for U strips.

\subsection{Event Reconstruction}

The MINOS detectors record neutrino interactions from NuMI beam spills and from muons induced in the surrounding rock as fyell as cosmic ray muons whose tracks have 

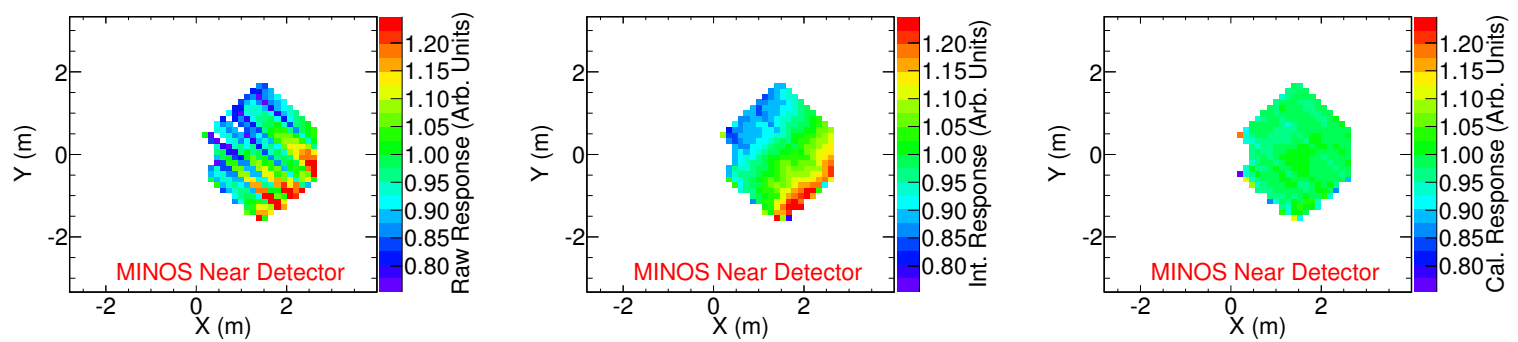

Figure 4.5: Response of the Near Detector in ADCs(left), in SigCors(middle) and in SigMaps(right), as a function of detector position, for U strips.
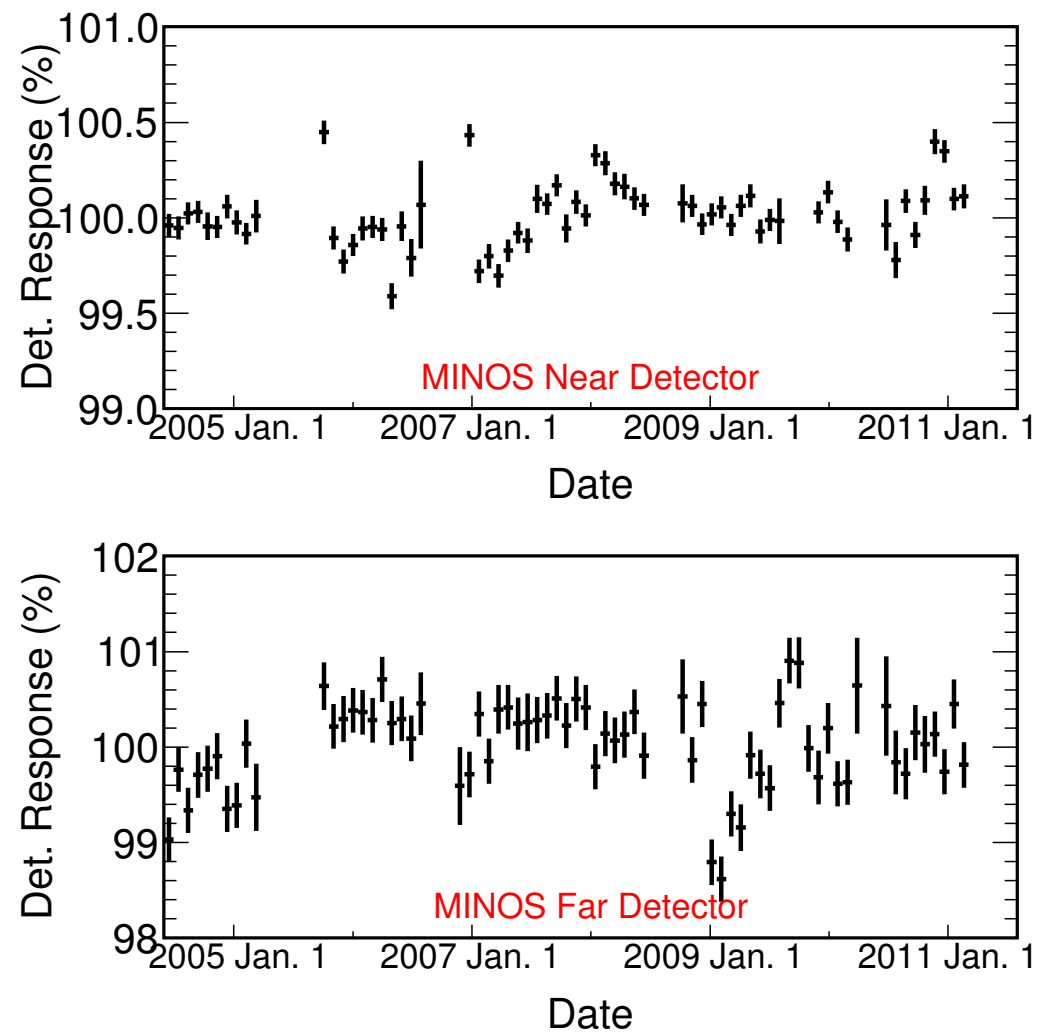

Figure 4.6: Far (above) and Near (below) detector MEU as a function of time, as measured using cosmic data. Individual runs outside of the Run period boundaries were removed. Each point corresponds to one month's worth of data. Each point is normalised to the overall MEU for the whole period.

traversed the detector. The reconstruction software uses the low level information such as hit topology and timing information contained in real or simulated raw data to reconstruct high level objects, such as muon tracks and hadronic showers. The input to the reconstruction chain is a snarl, and, in the ND, it typically contains several neutrino interactions. Each 
snarl corresponds to a beam-spill window or other trigger. The first step is to separate the individual neutrino interactions both spatially and temporally, allowing overlapping or simultaneous events to be distinguished. Fig. 4.7 shows a beam spill observed in the ND. A snarl is divided into individual slices based on the position and timing information of the hits. Tracks and showers are constructed from slices which are then assembled into events. Physical event properties such as position, vertex, length, angle and momentum of tracks, energy of showers are measured. An event may have more than one track or shower but one of each is defined as the primary track and shower. The event vertex is assigned as the first hit in the primary track.

Calibration, discussed in Section 4.1, aims to guarantee that the energy measurement is accurate and independent of time, in each strip of each detector and across the entire energy range. It also corrects for the relative near/far detector differences. This is particularly important for the oscillation analyses which obtain the FD prediction by extrapolating the ND data (see Sec. 5.3)
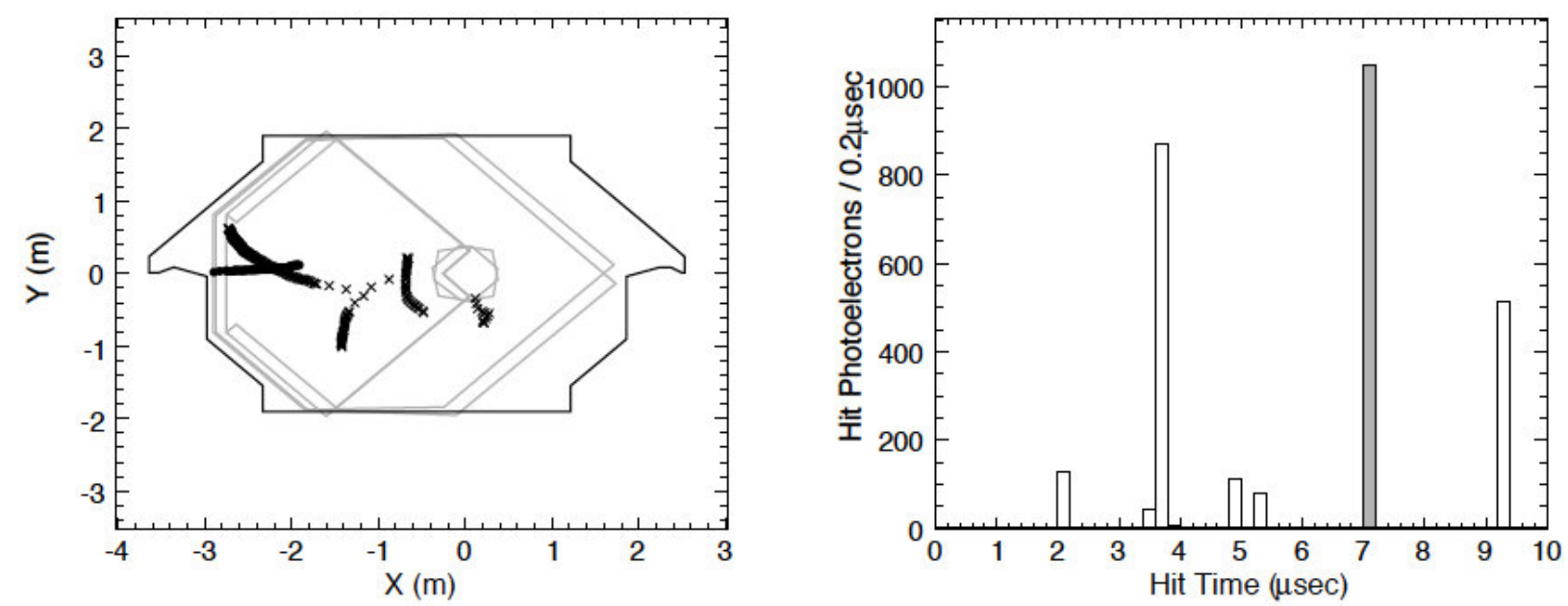

Figure 4.7: An example of a Near Detector snarl with several neutrino events distributed in space (left) and time (right). Figure from [112]. 


\subsubsection{Event Topologies}

MINOS beam events fall into four categories: charged current muon neutrinos $\left(\mathrm{CC} \nu_{\mu}\right)$, charged current muon antineutrinos $\left(\mathrm{CC} \bar{\nu}_{\mu}\right)$, charged current electron neutrinos/antineutrinos $\left(\mathrm{CC} \nu_{e} / \mathrm{CC} \bar{\nu}_{e}\right)$, and neutral current $(\mathrm{NC})$ events. There are also $\nu_{\tau}$ and $\bar{\nu}_{\tau}$ events, but they are very few and indistinguishable from neutral current events in the MINOS detectors.

The $\mathrm{CC} \nu_{\mu}$ and $\mathrm{CC} \bar{\nu}_{\mu}$ events are characterised by long, curving muon tracks $\left(\mu^{-}\right.$for $\nu_{\mu}$ and $\mu^{+}$for $\bar{\nu}_{\mu}$ ) with a hadronic shower at the interaction vertex. Such $\mathrm{CC} \nu_{\mu}$ and $\mathrm{CC} \bar{\nu}_{\mu}$ events can be distinguished by the direction of the track curvature in the detector magnetic field. While running with neutrino-mode beam, both detector fields are typically tuned to bend $\mu^{-}$inwards and $\mu^{+}$outwards, and vice versa during antineutrino-mode beam. The detector fields are chosen this way because tracks bent inwards are less likely to exit the detector, allowing the more accurate range momentum measurement to be used. $\mathrm{CC} \nu_{e} / \mathrm{CC} \bar{\nu}_{e}$ and NC interactions produce events without muon tracks, though they sometimes still have short reconstructed tracks. $\mathrm{CC} \nu_{e} / \mathrm{CC} \bar{\nu}_{e}$ events are characterised by compact electromagnetic showers and NC events are characterised by more diffuse hadronic showers. Specific analyses have been performed for both the $\mathrm{CC} \nu_{e} / \mathrm{CC} \bar{\nu}_{e}$ sample [113] and the NC sample [114], but in the analyses presented in this thesis it is not necessary to distinguish between $\mathrm{CC} \nu_{e} / \mathrm{CC} \bar{\nu}_{e}$ and NC events. Fig. 4.8 shows event displays from a $\mathrm{CC} \nu_{\mu}$, a $\mathrm{CC} \bar{\nu}_{\mu}$ and an NC interaction.

\subsubsection{Slice Formation}

In the ND we expect multiple neutrino interactions per snarl, which poses a challenge in separating individual showers and events. Therefore, the first step in the reconstruction chain is dividing the snarl into slices, which are clusters of hits localised in space and time. Calorimeter strips with charge deposition greater than two photoelectrons (pe), a time gap between two strips of shorter than 20 ns and a total time span of less than $300 \mathrm{~ns}$ are formed into a slice. Slices must contain at least two strips. If the slice thus formed has two strips with a spatial gap of greater than $1 \mathrm{~m}$ in the longitudinal direction, the slice is split into two 


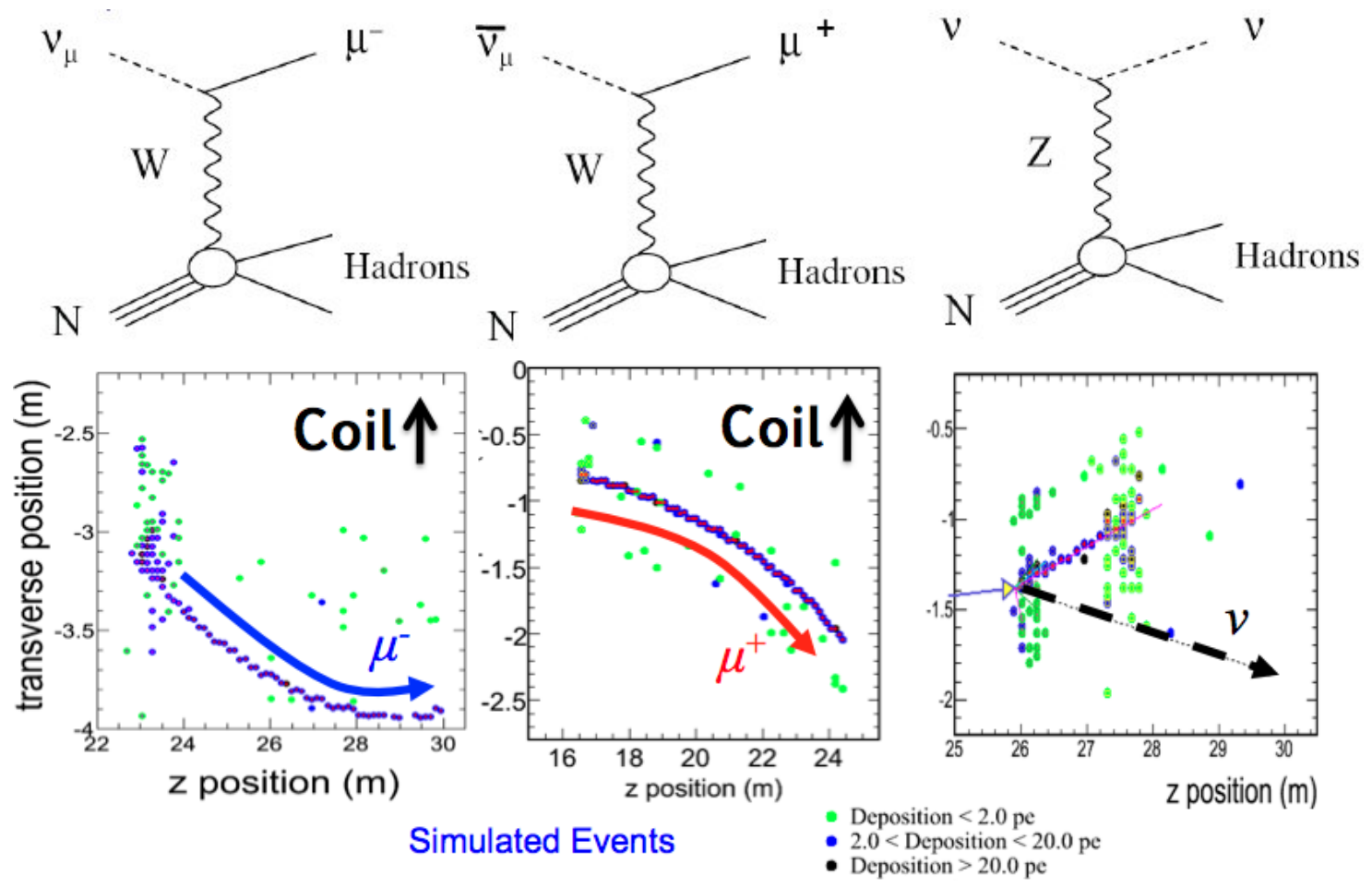

Figure 4.8: The three event topologies relevant to the antineutrino analysis: $\mathrm{CC} \nu_{\mu}$ (left), $\mathrm{CC} \bar{\nu}_{\mu}$ (centre), and NC (right). The top row shows the Feynman diagram and the bottom row shows a representative simulated event in one view (i.e. only $U$ planes). CC events are characterised by long muon tracks which curve in opposite directions for $\mathrm{CC} \nu_{\mu}$ and $\mathrm{CC} \bar{\nu}_{\mu}$. NC events do not have true muon tracks, but can have fake tracks which make them a background at low energy. The green points are hits with light levels below two photo-electrons and are not included in the analysis.

provided it doesn't span the coil hole. Calorimeter strips with a charge smaller than 2 pe and spectrometer strips are added to the most suitable slice based on timing information. The FD snarls are also sliced into multiple events, but the event rate is low enough that only one slice will typically be formed.

\subsubsection{Track Reconstruction}

Once the snarl has been split up into slices, the next step is the application of a trackfinding algorithm. This algorithm uses a Hough transform [115] to find track segments consisting of several hits in an approximate line across several detector planes. The track finder joins these segments to form a 'seed track'. The seed track is fit using a multi-pass 
Kalman Filter [116]. The filter works by moving forwards and backwards along the track estimating the state of the muon at each point along the track. It includes the effects of noise and multiple scattering in its estimate as well as the expected curvature in the magnetic field. Based on this information the filter decides whether or not the hit is a part of the track. After two passes with the filter, the track momentum (or more precisely, charge-to-momentum ratio $(\mathrm{q} / \mathrm{p}))$ is obtained from fitting the trajectory of the track. Other track properties are also measured at this time in addition to the momentum, such as track beginning and end positions in two views and the uncertainty in the charge/momentum measurement. At $3 \mathrm{GeV}$ the resolution of this measurement is $11 \%$.

If the track ends in the detector a second, more accurate measurement of its momentum is obtained from range. This measurement has a resolution of $4.6 \%$ and is used for all tracks that do not exit the detector or end in the uninstrumented coil hole region. This task is performed by the swimmer which swims a muon backwards along the track from the track end to the beginning and summing the energy it should have lost in the steel and scintillator using the GEANT3 simulation [117].

Muons with energies between $10 \mathrm{MeV}$ and $10 \mathrm{GeV}$ lose energy through ionisation which is well described by the Bethe-Bloch equation [118, 116]. This is precisely the energy regime relevant to oscillations in MINOS. CalDet demonstrated the accuracy of the Bethe-Bloch equation as tabulated by Groom [116] with material-specific density effects tabulated by Sternheimer [119, 120]. The uncertainty between data and simulation was calculated to be $2 \%$ by incorporating the Groom tabulation into GEANT3. This is taken as the systematic uncertainty on the measurement from range. Since $95 \%$ of the energy loss occurs in the steel planes, each of which is nominally 1.46 radiation lengths thick, the amount of steel the muon passes through must also be known precisely. The density of the steel was measured to an accuracy of $0.3 \%$ and the near and far detector plane thicknesses were measured to $0.1 \%$ and $0.2 \%$ respectively.

The curvature-based measurement of the track momentum was calibrated by compari- 
son with the range-based measurement. The two energy measurements were compared for stopping tracks, and it was found that the curvature-based measurement generally agreed with the range-based measurement to within $1 \%$. This $1 \%$ is conservatively added linearly to the $2 \%$ uncertainty from the range measurement, leading to a total uncertainty of $3 \%$.

\subsubsection{Cluster Formation and Shower Reconstruction}

Once the track is identified, the remaining hits localised in space and time are clustered together to construct the shower. Hits that are part of a track but have more energy than what the muon would have deposited are added to the shower after the muon part is removed. Only hits with an energy deposition of greater than two photoelectrons are considered since the low pulse height region is difficult to model. The shower energy cannot be reconstructed topologically since the detector granularity is too coarse to distinguish individual components of a shower. There are two methods to measure the shower energy described below.

One method of measuring the shower energy is calorimetry where the energy of the shower is calculated by summing the energy deposited by all of its constituent hits (after the energy deposited in the steel is recovered). By this method, the hadronic shower energy resolution is $56 \% / \sqrt{E} \bigoplus 2 \%$ and the electromagnetic shower energy resolution is $21.4 \% / \sqrt{E} \bigoplus 4 \%[105]$.

Another way of measuring shower energies is the $k$-nearest neighbors $(k \mathrm{NN})$ method, which employs a multivariate neural network technique to improve the energy resolution of the showers, making use of a broader range of information about the shower, and the event as a whole. With this method, shower energies are reconstructed by using the amount of deposited energy as well as the spatial distribution of energy deposition in the shower. The method is presented in detail in [121] and is summarised here.

To implement the $\mathrm{kNN}$ algorithm in estimating shower energy four elements are required: the training set, the variables for building the multi-dimensional feature space, the number of nearest neighbors and an energy correction function. The algorithm begins by taking a training sample of Monte Carlo events which have passed the charged-current selection, dis- 
tributed across the run periods in the correct proportions to accurately reflect the properties of the data. Separate samples are used for the near and far detectors to allow for interdetector differences. For a $D$-dimensional $k \mathrm{NN}$, a set of $D$ variables is considered which characterise the properties specific to the shower component of an event and which correlate with the true shower energy. The distribution of Monte Carlo events in this $D$-dimensional space is known, since their true shower energies as well as the true values of the $D$ variables are known. The $k$ nearest neighbors of the query event, whose shower energy is being estimated, are found by computing its Euclidean distance to each of its neighbors and taking the $k$ closest ones. Let $\boldsymbol{y}$ be the query event we seek to identify and $\boldsymbol{x}$ the Monte Carlo event whose true parameters are known. The squared distance between the two is given by

$$
\Delta s^{2}=\sum_{D}^{i=1} \frac{\left(y_{i}-x_{i}\right)^{2}}{\sigma_{i}^{2}},
$$

where $\sigma_{i}$ is the standard deviation of the variable $i$. The normalisation by the standard deviations is necessary to ensure consistency in the scales of the variables. The scale differences can be large since the variables are not necessarily in the same units, and if they are not normalised the one with the widest numerical range would dominate.

To estimate the shower energy of a candidate event, the $k$ closest training events are found in the D-dimensional space formed by the input variables. The output energy estimate is then the mean of the true shower energies of these training events, illustrated in Fig. 4.9.

The number of input variables, $D$, and the number of nearest neighbors, $k$ were optimised to maximise the sensitivity to oscillations [121], and the $k \mathrm{NN}$ selector with $D=3$ was selected. The three variables are:

- Calorimetric energy in the first two showers,

- Total energy deposited within a $1 \mathrm{~m}$ radius of the track vertex,

- Number of planes in the primary shower.

The same set of three variables is used for energy estimation in both the near and far 


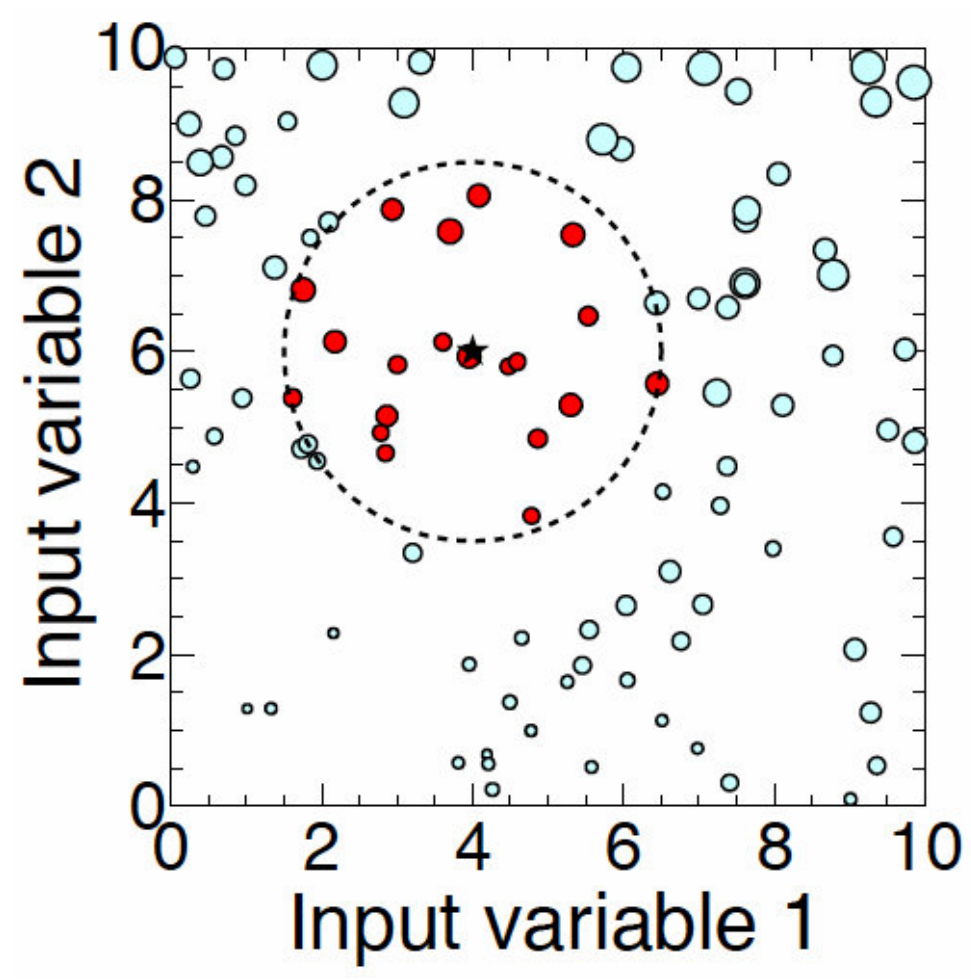

Figure 4.9: Illustration depicting the use of the kNN technique for shower energy estimation. In this two-dimensional space, higher energy training events (represented by larger circles) correlate with larger values of the variables. The energy of the event marked by the star is estimated by examining its k (here 20) nearest neighbours, highlighted in red. The energy estimate is the mean of the true energies of these neighbouring events.

detectors, although based on training samples drawn from the respective Monte Carlo samples. Studies with varying the number of $k$ neighbors yielded that the optimal number of neighbors with maximum sensitivity for the size of the Monte Carlo sample used is $k=400$.

\subsubsection{Event Formation}

Event formation is the stage where reconstructed tracks and showers are combined to form candidate neutrino events. The final event object is therefore the combination of the vertex shower and the most energetic muon track. The optimal combination of objects is identified by considering the temporal and spatial distribution within each slice. For muon neutrino interactions, the neutrino energy is estimated to be the sum of the energies of the longest track and the vertex shower. The presence of a track with a clearly defined vertex is 
a condition applied when selecting a $\nu_{\mu}$ or $\bar{\nu}_{\mu}$ sample, in addition to cuts on other quantities used to remove NC events where short tracks are mistakenly reconstructed within showers (see Chapter 4)

\subsection{Monte-Carlo simulation}

The two-detector design greatly reduces the dependence of the MINOS analyses on the MC simulation. Still, it is necessary to have a MC simulation that reproduces the data as accurately as possible. The simulation needs to be used to predict the FD spectrum from the measured ND spectrum, as well as to perform systematic studies. The MINOS data is simulated in a multi-stage process that begins with the beam simulation using the FLUGG MC generator [122, 123, 124]. The FLUGG package incorporates a geometry based on GEANT4 [125] into a FLUKA [126, 127] simulation of the hadronic production, decay and transport processes [128]. The simulation begins with the production of secondary mesons from Main Injector protons incident on the graphite target. The mesons are transported through the magnetic horns, into the decay pipe, and all other further downstream devices and materials. Every hadronic decay that produces a neutrino is stored and later used for the simulation of neutrino interactions. Based on the position and momentum of a neutrino, its energy and the probability that it reaches the detector is calculated. Every neutrino is forced to go through the detectors but with a weight based on its probability of actually doing so. The resulting weighted neutrino flux is then passed to the MINOS detector simulation.

Neutrinos are sampled from the flux simulation, using a technique known as "importance sampling" or "weighting" to reduce the number of low energy particles that are produced. The simulation produces many more low energy mesons than high energy ones, which makes it difficult to collect enough statistics at higher energies. To increase the number of high energy events relative to the low energy ones, a $1 \mathrm{GeV}$ tracking threshold is imposed, since those mesons will produce approximately neutrinos of $500 \mathrm{MeV}$ or lower, which is the lowest energy that MINOS detectors are sensitive to. Events with energy less than $1 \mathrm{GeV}$ are 
discarded. Even then too many low energy particles survive. To speed up the simulation and to reduce the space required on disk, pions with energy less than $30 \mathrm{GeV}$ are importance sampled and weighted. This means that the particles having energy below the threshold of $1 \mathrm{GeV}$ are discarded and the remaining particles are given a weight greater than one so that the total flux after reweighting stays the same; $\nu_{\mu}$ 's, $\mu$ 's, and $K$ 's are not given any additional weight. The weight $W$ is calculated as,

$$
W=W_{\text {parent }} \frac{30 G e V}{\left|P_{\text {total }}\right|}
$$

where $W_{\text {parent }}$ is the importance weight of the particle's parent (initial protons start with a weight 1 ), and $P_{\text {total }}$ is the total momentum of the particle. The weights are constrained to lie between 1 and 100 .

The neutrinos obtained after the rejection sampling are traced through models of the near and far detector halls, allowing the neutrinos to interact both in the detector and in the surrounding material. The neutrino interactions are generated by the NEUGEN [129] neutrino event generator. NEUGEN simulates quasi-elastic scattering (QE), resonance production (Res), deep-inelastic scattering (DIS) and coherent pion production (Coh) processes in an energy range from $100 \mathrm{MeV}$ to $100 \mathrm{GeV}$. The hadronisation is simulated with the AGKY model [130], which uses a combination of PYTHIA/JETSET [131] at high hadronic invariant mass and the KNO phenomenological model [132] at low invariant mass, with a smooth transition between the two models. NEUGEN also includes the INTRANUKE [133] model of intranuclear rescattering to account for the interactions of the hadronic particles as they leave the nucleus.

Once the products of the interaction leave the nucleus, their simulation is taken over by GMINOS which includes a detailed geometric model of the detector written in GEANT3 [117. It also includes a detailed model of the magnetic field created using finite element analysis and measured B-H curves from steel samples [105]. GMINOS transports the parti- 
cles through the detector geometry, recording their strip-by-strip energy depositions as the particles lose energy into the steel and scintillator. At this stage, multiple neutrino interactions, both from in the detector and in the surrounding material, are overlaid into a single simulated snapshot in time to reflect the high ND event rate.

After the energies have been deposited on scintillator strips, the $\mathrm{C}++$ based PhotonTransport program takes over. PhotonTransport generates photons in the scintillator based on the GMINOS energy depositions, transports those photons into the WLS fibre and onto the PMT cathode where they are converted into photoelectrons. It includes the detailed behaviour of the PMTs and electronics, including non-linearity, noise, cross-talk, and triggering. Thus the simulation includes the best knowledge available on light levels, attenuation, non-linearity and gains. Each simulated run is given a fictitious date from some time during actual data taking and calibration constants from that time are used. Later, when calibrations are re-applied, each Monte Carlo run is re-calibrated using the same data that was used to produce it.

At this stage, the simulation is as similar to the real data as possible and both are handled in the same way during reconstruction.

\subsection{Analysis Dataset}

MINOS has accumulated $15.6 \times 10^{20}$ POT between 2005 and 2012 . Of this $10.56 \times 10^{20}$ POT was obtained in the low energy (LE) neutrino-dominated beam mode. An additional $0.15 \times 10^{20} \mathrm{POT}$ was also obtained in the neutrino-dominated mode but with a $10 \mathrm{GeV}$ beam peak. About $3.36 \times 10^{20}$ POT was obtained in the LE antineutrino-enhanced beam mode. Fig. 4.10 shows the POT accumulated per week $\left(\times 10^{18}\right)$ and the total accumulated POT $\left(\times 10^{20}\right)$ for the entire MINOS running.

The analyses described in this thesis use the data accumulated in the LE neutrinooptimised beam running shown by the green shaded area in Fig. 4.10. The first analysis (Chapter 5), which studies the antineutrino component in the neutrino-optimised beam, 
Total NuMI protons to 00:00 Monday 07 May 2012

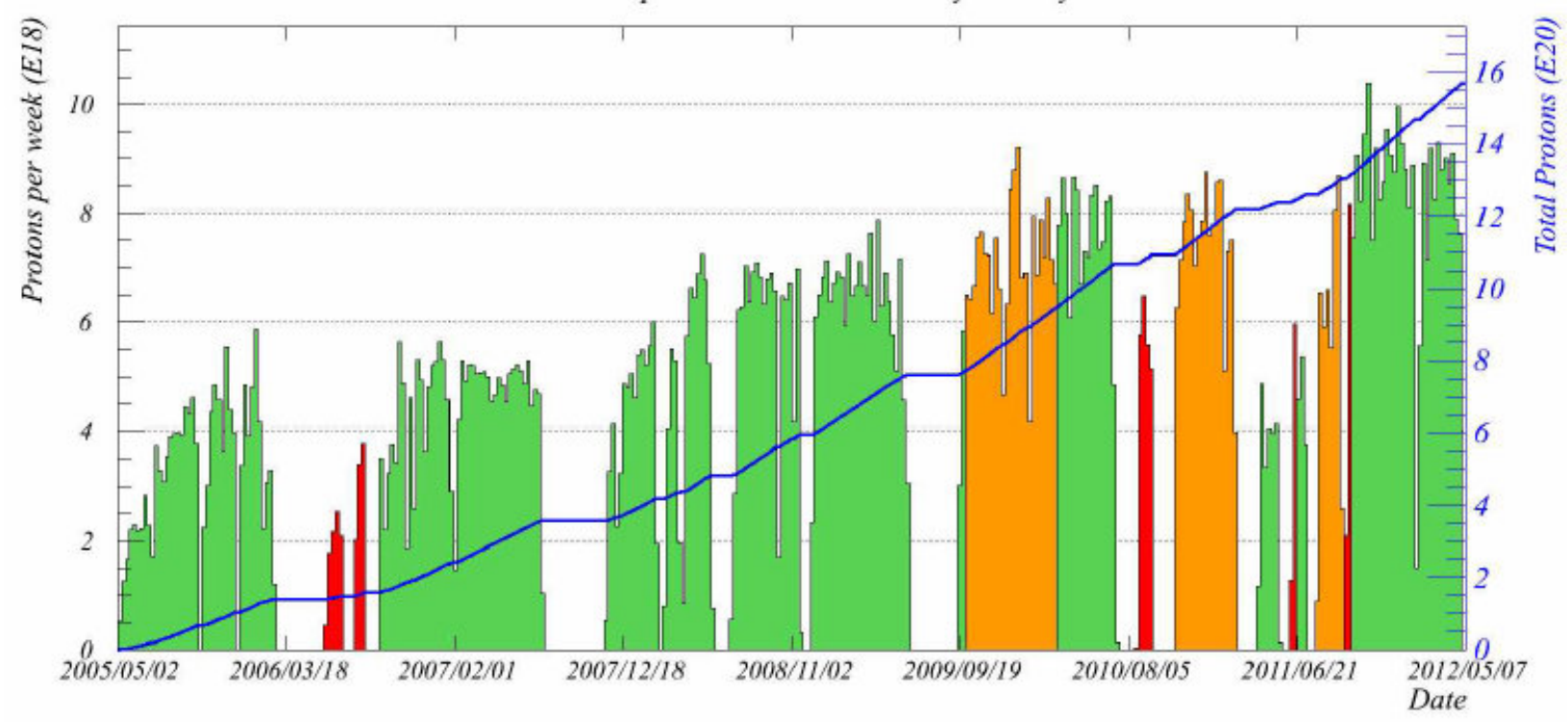

Figure 4.10: The accumulated POT as a function of time for all MINOS running. The dominant running mode is low energy neutrino production in the forward horn current (FHC) configuration, shown in green. The antineutrino production mode, using the reversed horn current (RHC) configuration is indicated in orange. The higher energy modes, at different target positions, is shown in red.

uses the data obtained in a $7.1 \times 10^{20}$ POT exposure collected between May, 2005 and September, 2009. The second analysis (Chapter 6), which looks for antineutrino appearance because of neutrino to antineutrino oscillations, uses all the data in a $10.56 \times 10^{20} \mathrm{POT}$ exposure accumulated in the LE configuration. 


\section{Chapter 5}

\section{Antineutrinos in a Neutrino Beam}

The first $7.1 \times 10^{20}$ POT of data were taken with the NuMI beamline running in neutrinodominated mode. In this mode the beam has a small (approximately $7 \%$ ) component of antineutrinos. The disappearance analysis measures the $\Delta \bar{m}_{32}^{2}$ and $\sin ^{2} 2 \bar{\theta}_{23}$ parameters from the oscillation survival probability in a two-flavour approximation:

$$
P\left(\bar{\nu}_{\mu} \rightarrow \bar{\nu}_{\mu}\right)=1-\sin ^{2} 2 \bar{\theta}_{23} \sin ^{2}\left(\Delta \bar{m}_{32}^{2} \frac{L}{4 E}\right)
$$

where $L$ is the length over which the neutrino travels and $E$ is the energy of the neutrino.

As explained in 3.1 the antineutrino component in the FHC running comes primarily from negatively charged mesons travelling through the centre of the magnetic horns that do not get deflected. Due to this reason the antineutrinos have a higher energy as compared to the neutrinos. The ND spectra of muon antineutrinos in FHC-mode is shown in Fig. 3.4 (shown in red).

For an oscillation analysis, to begin with, the ND and FD energy spectra are selected. A measurement of neutrino spectrum at the ND enables us to make a prediction at the FD in case of no oscillations. These two spectra are closely related but are not identical. In addition to having lower statistics because of being further away from the neutrino source, the FD also has a different spectral distribution due to the geometry of the beamline and 
the meson decay kinematics. We use the Monte Carlo to account for these differences and make predictions for FD spectrum in case of no oscillations or oscillations. This procedure is called extrapolation. To measure antineutrino oscillation parameters the FD observed event rate as a function of energy is compared with prediction. Using two-flavour oscillation model given by equation 5.1 we fit for mixing angle and mass splitting between the second and third mass states.

\subsection{Event Selection}

The ND and FD spectra are obtained by requiring that the events satisfy a series of selection criteria. The aim is to select the largest possible sample of antineutrinos with the least possible background contamination. Since the antineutrino itself cannot be observed directly its properties are inferred from the outgoing particles produced when an antineutrino interacts with nuclei in the detector. We use only charged-current (CC) interactions for this analysis since the neutrino flavour can be determined based on the outgoing lepton.

$$
\bar{\nu}_{\mu}+N \rightarrow \mu^{+}+\text {hadrons }
$$

The magnetic field of the MINOS detectors is used to distinguish neutrinos from antineutrinos. The event topologies resulting from $\nu_{\mu}$ and $\bar{\nu}_{\mu} \mathrm{CC}$ interactions are described in Sec. 4.2.1. The separation of neutrinos and antineutrinos relies on the fact that the two CC interactions produce opposite sign outgoing leptons: $\bar{\nu}_{\mu} \rightarrow \mu^{+}$and $\nu_{\mu} \rightarrow \mu^{-}$. The torroidal magnetic fields (approximately $1.3 \mathrm{~T}$ ) in the two detectors cause the outgoing muons to curve in different directions depending on their charge. When the detectors are in neutrinomode negative muons are focused and positive muons are defocused by the magnetic field in the detectors and vice-versa. The reconstruction algorithm measures the track's curvature, which is proportional to the ratio of the tracks's charge/momentum $(q / p)$.

There are two main backgrounds to the $\mathrm{CC} \bar{\nu}_{\mu}$ sample: $\mathrm{CC} \nu_{\mu}$ interactions whose charge 
has been mis-reconstructed (called ‘wrong sign' or WS)

$$
\nu_{\mu}+N \rightarrow \mu^{-}\left(\text {misidentified as } \mu^{+}\right)+\text {hadrons }
$$

or neutral current (NC) interactions of any neutrino species which have a shower element that is misidentified as a muon track

$$
\nu_{x}+N \rightarrow \nu_{x}+\text { fake muon }+ \text { hadrons. }
$$

\subsubsection{Preselection}

To select the desired events the first step is to apply basic preselection cuts. It is required that the accelerator and detector must be in good operating condition. The NuMI should be operating in the low-energy mode for which the target is inside the first horn, the separation between the two horns is $10 \mathrm{~m}$ and the horn current is $185 \mathrm{kA}$. Both detector coils need to be ON so that the detectors are magnetised. The event must occur within a beam spill so that atmospheric and cosmic backgrounds are reduced. Events must have at least one muon track to eliminate most NC backgrounds. Track vertices are required to lie within the fiducial volume of each detector to ensure that all the energy of the event is fully contained in the detector and can be measured.

\subsubsection{Selection}

The next step in selecting antineutrinos is to keep only events with positive reconstructed charge. However, the antineutrino component in the beam is so small that even this selected sample is dominated by backgrounds (see Fig. 5.1). The backgrounds are neutral current events and wrong-sign neutrinos. The positive sample consists of at least $50 \%$ background at all energies, and the contamination increases below $7 \mathrm{GeV}$ which is the region of interest for oscillations analysis.

An additional selection step is applied for each of these backgrounds. Two cuts are 
made to reduce the wrong-sign background, and a third cut is made to reduce the NC background. The two charge-sign selection variables are the ratio of the track's curvature $\left(\frac{q}{p}\right)$ to the uncertainty on that curvature $\left(\sigma\left(\frac{q}{p}\right)\right)$, and the relative angle between the straightline projections of the first few hits and the last few hits of the track. The distributions of these two variables are shown in Fig. 5.2. The CC/NC separation parameter, called $D p I D$, is built up from the following 1-dimensional PDFs of three variables that each have some power to distinguish charged current events from neutral currents:

- Track length: True muon tracks tend to be longer (e.g. cross more planes) than the tracks of particles coming out of the hadronic shower.

- Track energy fraction: The fraction of the event energy contained in the track (lepton) as compared to the hadrons (shower). It is related to the kinematic $y$ (inelasticity) distribution.

- Track energy per plane: The amount of energy deposited per plane of the track. It is

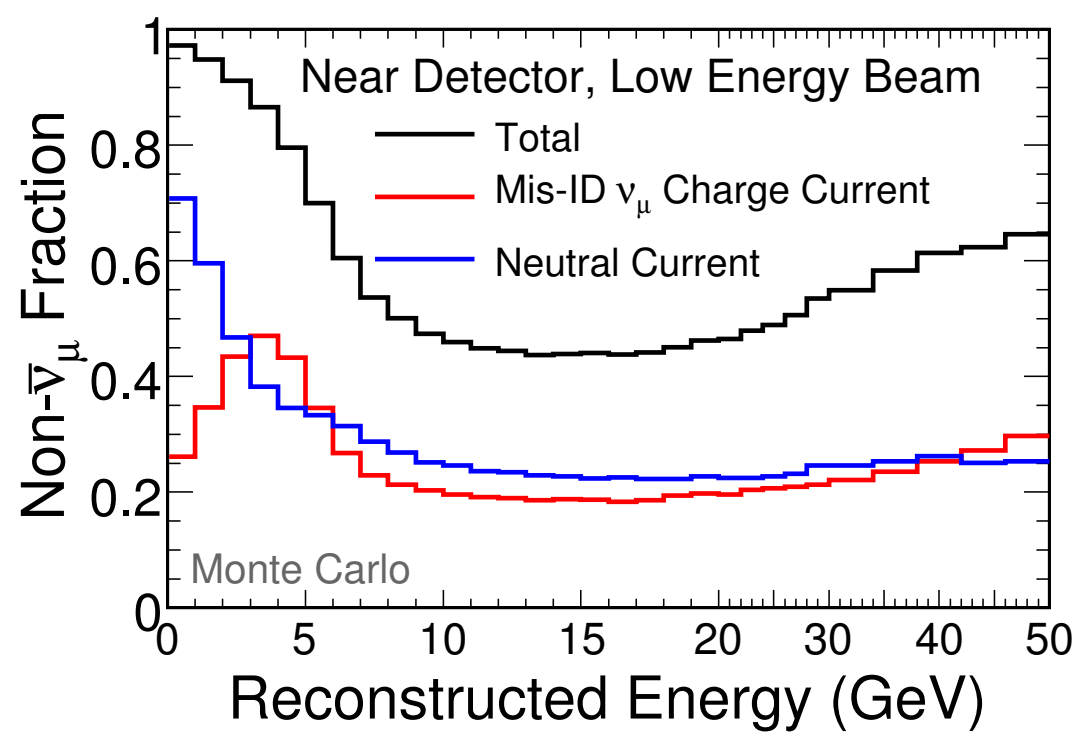

Figure 5.1: Contamination in the positive sample after preselection. The black line represents the total contamination (1-purity), the red line represents the fraction of events that are mis-identified $\nu_{\mu} \mathrm{CC}$ (wrong sign) and the blue line represents the fraction of events that are neutral currents. 

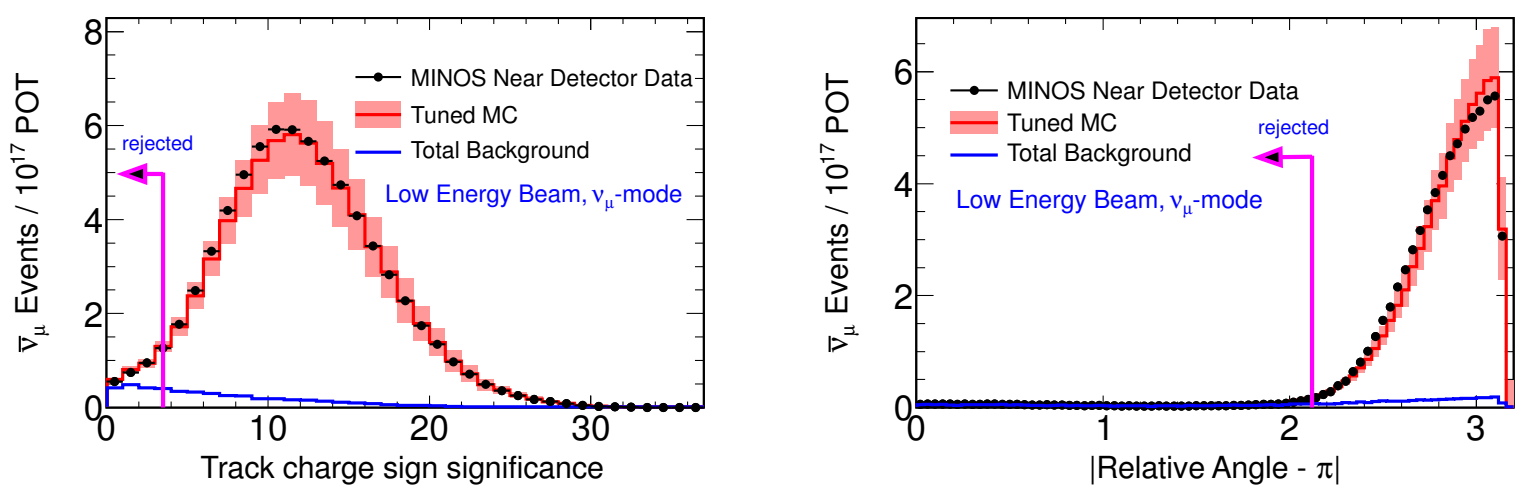

Figure 5.2: Charge-sign selection variables $\frac{\frac{q}{p}}{\sigma\left(\frac{q}{p}\right)}$ and RelativeAngle are shown for the ND in data (black points) and simulation (total in red, background in blue). The total systematic uncertainty on the Monte Carlo is represented by the shaded red bars. In each plot, all other selection cuts have been applied.

related to $d E / d x$ which can distinguish true muons (typically minimum-ionising) from the tracks formed by hadronic shower components.

These three variables for the ND data and simulation are shown in Fig. 5.3. The distribution of the separation parameter for the ND data is shown in Fig. 5.4. In addition to removing neutral current events, $D p I D$ is also effective at removing many wrong-sign events because one of its input PDFs is related to inelasticity (kinematic $y$ ) and neutrino interactions typically have a higher $y$ than antineutrinos. Fig. 5.5 shows the performance of the antineutrino selection in purity and total (reconstruction and selection) efficiency as well as the purity in the positive sample before selection (as shown in Fig. 5.1). The three selection cuts on charge-sign and the CC/NC separator improve the purity of the sample from $34 \%$ to $99 \%$ while keeping the overall efficiency at $85 \%$ in the ND.

\subsection{Near Detector Spectrum}

The energy spectrum at the Near and Far Detectors can be built up by combining the track and shower energy for each event. The distributions for track and shower energies for the ND are shown in Fig 5.6. The selected antineutrino energy spectrum at the ND in the 

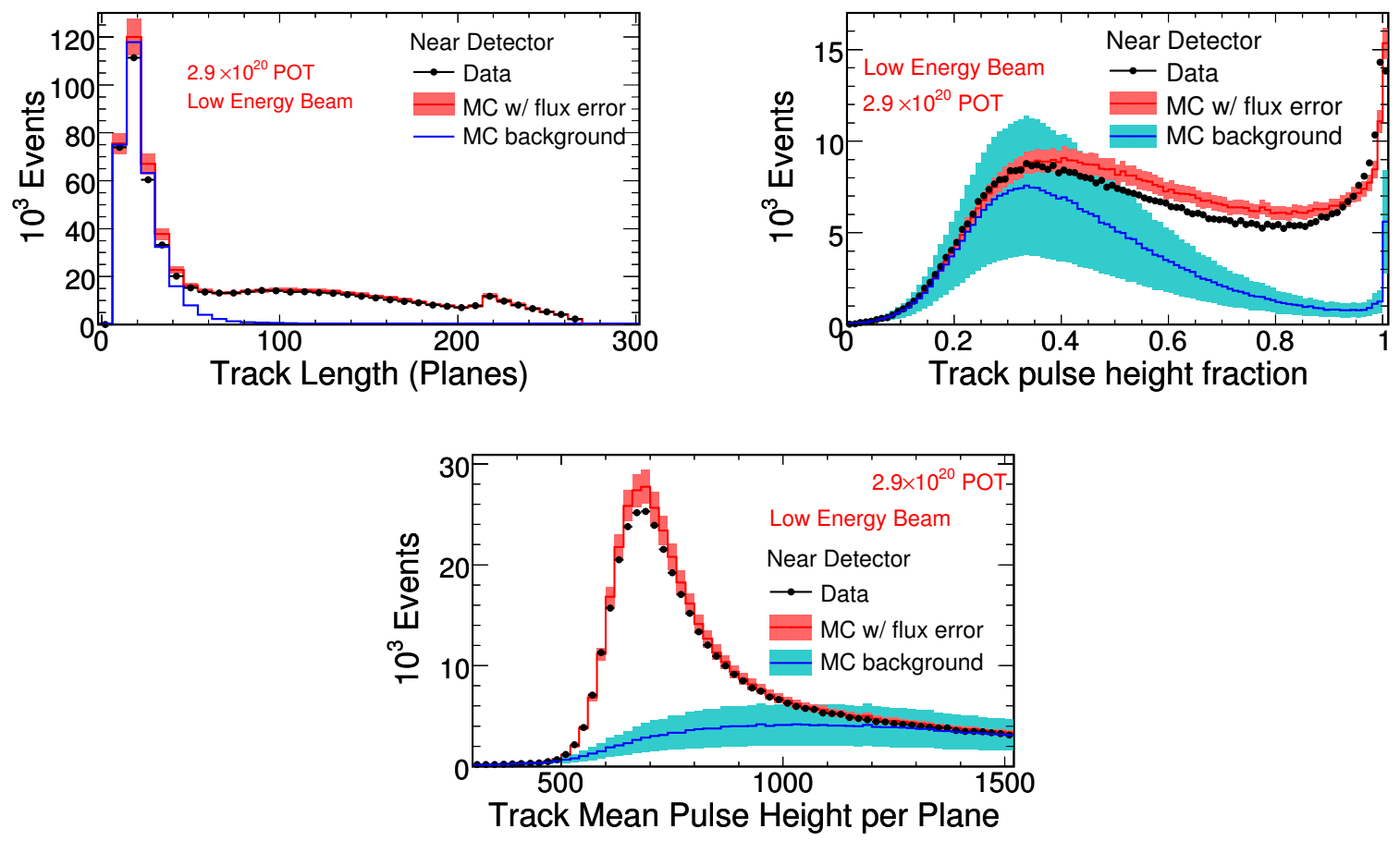

Figure 5.3: The three variables that form the PDFs in the $D p I D \mathrm{CC} / \mathrm{NC}$ separator: the track length, the fraction of the event energy in the track, and the mean energy deposited per plane are shown for the ND in data (black points) and simulation (total in red, background in cyan). The flux uncertainty on the Monte Carlo is represented by the shaded red bars and the background systematic uncertainty is represented by the shaded blue bars.

FHC beam can be seen in Fig. 5.7. The data and Monte Carlo are shown along with the systematic uncertainty in the Monte Carlo. Background is also shown in blue.

The simulation shown in Fig. 5.7 is not the raw Monte Carlo - it has been reweighted as a function of the neutrino parent's initial momentum $\left(\mathrm{p}_{\mathrm{T}}, \mathrm{p}_{\mathrm{Z}}\right)$ leaving the target to account for the mismodelling of hadronic interactions in the NuMI target. The NuMI beam has the flexibility to allow data collection in multiple beam configurations (see Fig. 3.6). By simulating the flux in various beam configurations and performing a fit to all the configurations simultaneously, the flux prediction was tuned to achieve good agreement with the observed ND data. The fit constrains the $\pi^{+} / \pi^{-}$ratio by using both simulated results from FLUKA05 and experimental results from the NA49 experiment [134]. The separate runs are also fitted simultaneously. The best fit is used to determine weights for the pions and kaons which are 


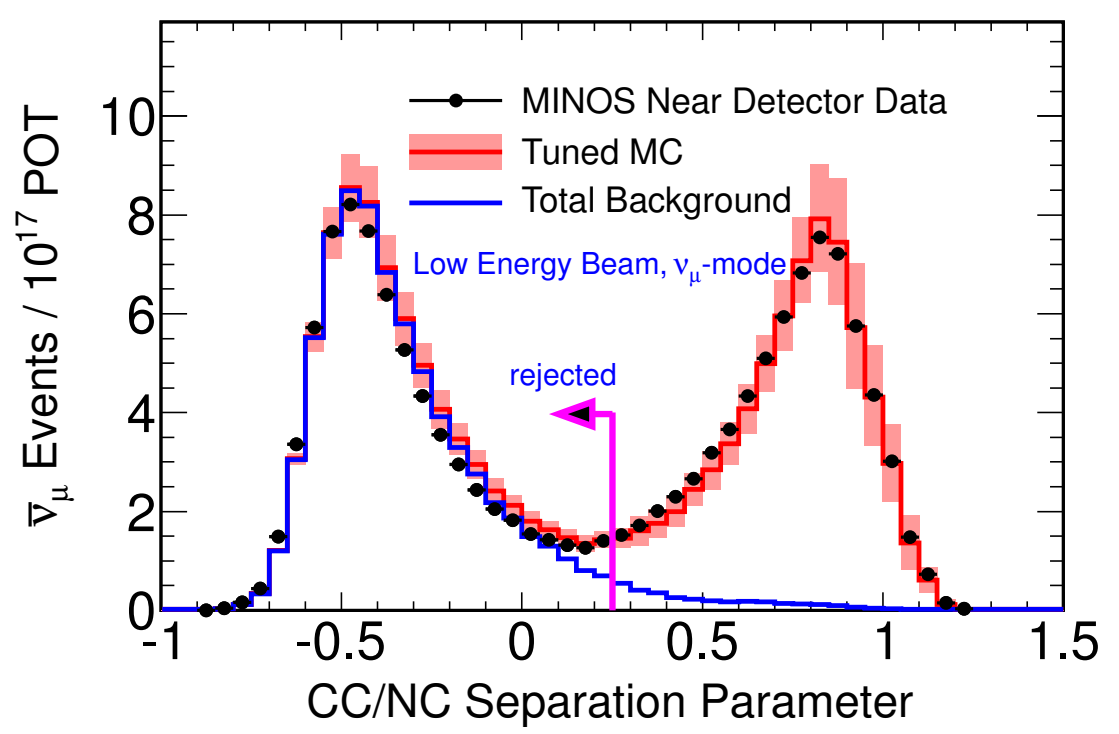

Figure 5.4: CC/NC separation parameter $(D p I D)$ is shown for the ND in data (black points) and total Monte Carlo with flux uncertainty (red line and shaded bars). Also shown is the total background in blue. The $\mathrm{CC} / \mathrm{NC}$ separator has some power to reject the wrong-sign background due to the higher average inelasticity $(y)$ of neutrinos compared to antineutrinos. All other cuts have been applied.

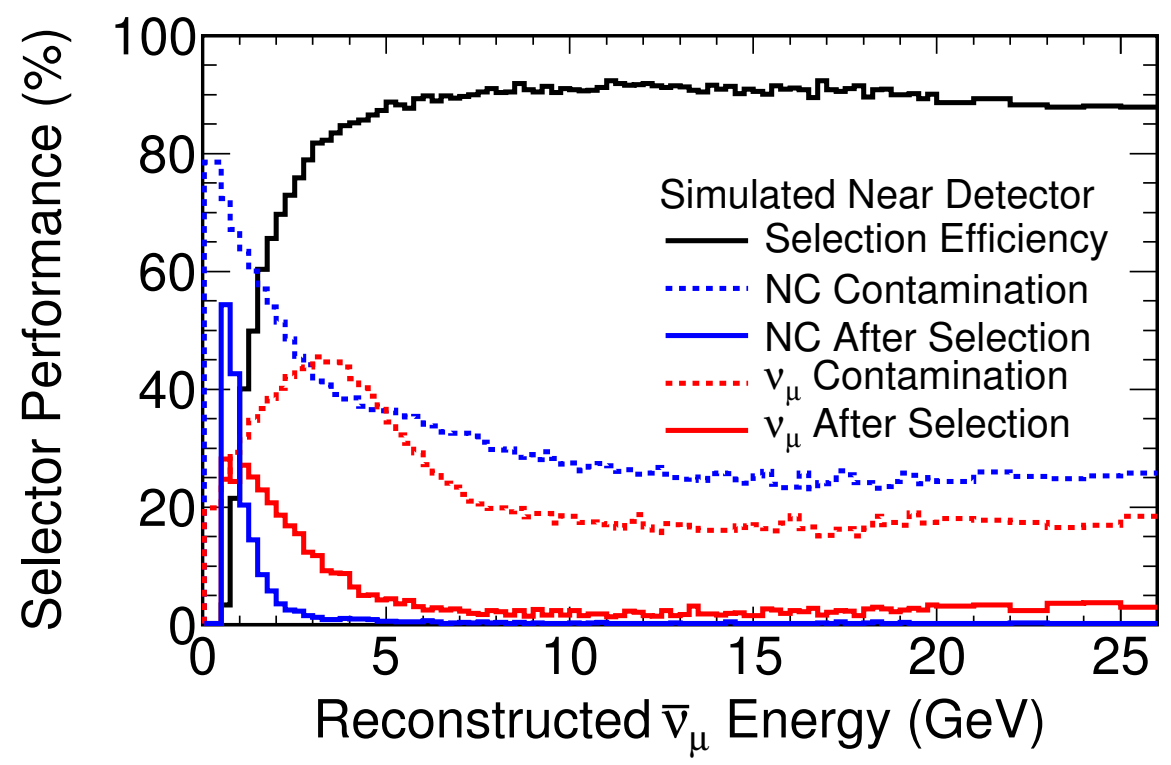

Figure 5.5: This figure shows the contamination in the sample of events with positive reconstructed charge. The dashed and solid lines respectively show the contamination before and after the selection cuts. NC background is in blue and wrong-sign background is in red. 

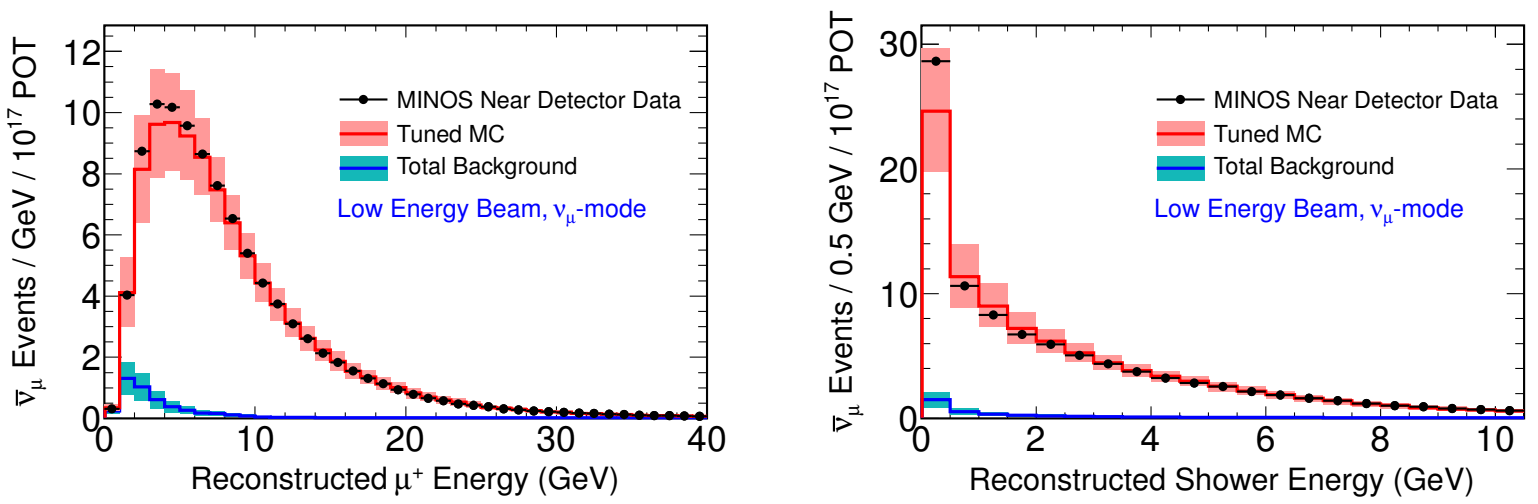

Figure 5.6: The track energy (left) and shower energy (right) distributions shown for the ND; data as black points and simulation (total in red, background in blue). The total systematic uncertainty on the Monte Carlo is represented by the shaded red bars.

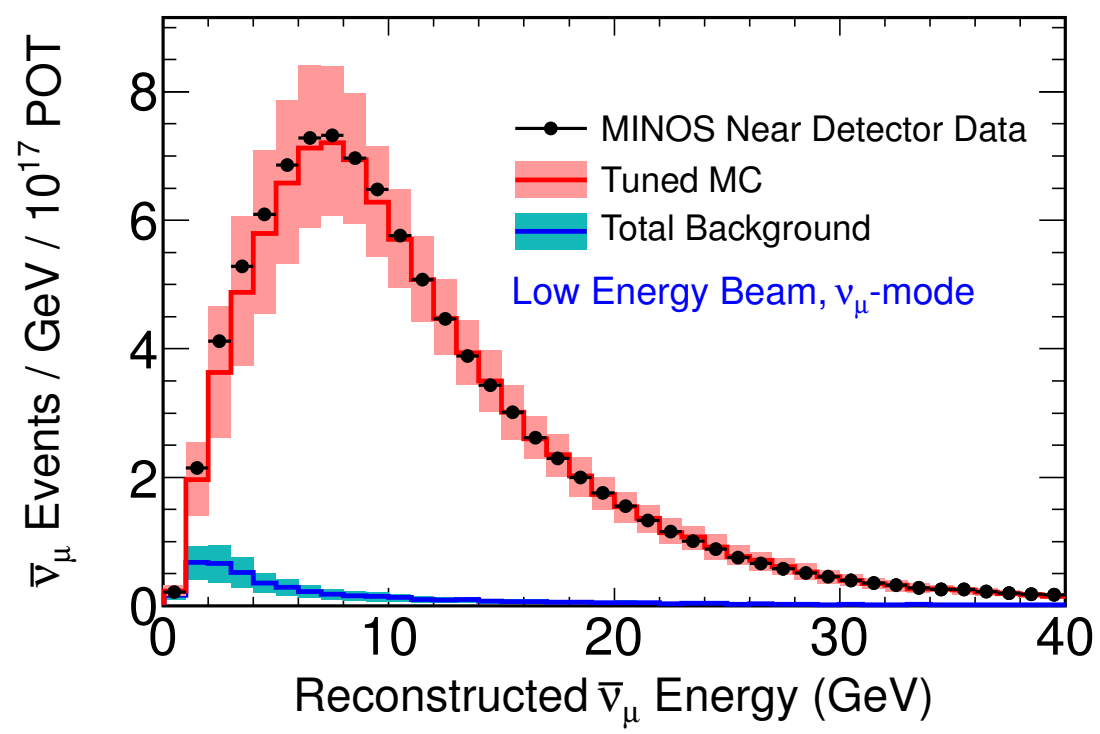

Figure 5.7: The selected $\bar{\nu}_{\mu}$ energy spectrum is shown for the ND; data is shown as black points, background as blue and tuned MC in red. The total systematic uncertainty on the Monte Carlo is represented by the shaded red bars.

propagated to the Near and Far Detectors.

The hadron production is parametrised following the BMPT parametrisation, [135],

$$
\frac{d^{2} N}{d x_{F} d p_{T}}=\left[A+B p_{T}\right] \cdot \exp \left(-C^{3 / 2} p_{T}\right)
$$


where $\mathrm{p}_{\mathrm{T}}$ is the transverse momentum of the meson and $\mathrm{x}_{\mathrm{F}}=\mathrm{p}_{\mathrm{Z}} / \mathrm{p}_{\text {proton }}$ is the ratio of its longitudinal momentum to the momentum of the proton. The $\mathrm{p}_{\mathrm{T}}$ distribution is fitted for different bins of $\mathrm{p}_{\mathrm{Z}}$ and the parameters $A, B$ and $C$ are allowed to vary in the fit and become functions of $\mathrm{x}_{\mathrm{F}}$. The reweighting is determined by fitting a paramaterised warping of the hadron production to the neutrino and antineutrino spectra in the ND. For $\pi^{+}, A, B$, and $C$ are redefined as,

$$
\begin{aligned}
& A^{\prime}\left(x_{F}\right)=\left(\operatorname{par}[0]+\operatorname{par}[1] \cdot x_{F}\right) \cdot A\left(x_{F}\right) \\
& B^{\prime}\left(x_{F}\right)=\left(\operatorname{par}[2]+\operatorname{par}[3] \cdot x_{F}\right) \cdot B\left(x_{F}\right) \\
& C^{\prime}\left(x_{F}\right)=\left(\operatorname{par}[4]+\operatorname{par}[5] \cdot x_{F}\right) \cdot C\left(x_{F}\right)
\end{aligned}
$$

and similarly for $K^{+}$with parameters $\operatorname{par}[6]-\operatorname{par}[11]$. The weight for a positive meson is calculated as,

$$
W\left(\pi^{+} / K^{+}, p_{T}, p_{Z}\right)=\frac{\left[A^{\prime}+B^{\prime} p_{T}\right] \cdot \exp \left(-C^{\prime 3 / 2} p_{T}\right)}{\left[A+B p_{T}\right] \cdot \exp \left(-C^{3 / 2} p_{T}\right)}
$$

and that for negative mesons as,

$$
\begin{aligned}
W\left(\pi^{-}, p_{T}, p_{z}\right) & =\left(\operatorname{par}[12]+\operatorname{par}[13] x_{F}\right) W\left(\pi^{+}, p_{T}, p_{Z}\right) \\
W\left(K^{-}, p_{T}, p_{z}\right) & =\left(\operatorname{par}[14]+\operatorname{par}[15] x_{F}\right) W\left(K^{+}, p_{T}, p_{Z}\right)
\end{aligned}
$$

Thus there are sixteen hadron production fit parameters. Besides these, there are eight additional parameters in the fit to account for uncertainties in the flux measured by the ND, such as focusing, detector effects and target degradation. Fig. 5.8 shows the ND data and Monte Carlo with and without this tuning. The tuned Monte Carlo is clearly a much better fit to the data which makes this and all other ND data-MC comparison plots easier to interpret. However, because of the cancellation of uncertainties in the flux when extrapolating, the beam reweighting does not affect the final result much. To study the effect of the reweighting, cross-check studies were performed. It was found that the FD prediction changes by at most 
a few percent at all energies, and even less at the lower energies relevant to oscillations.

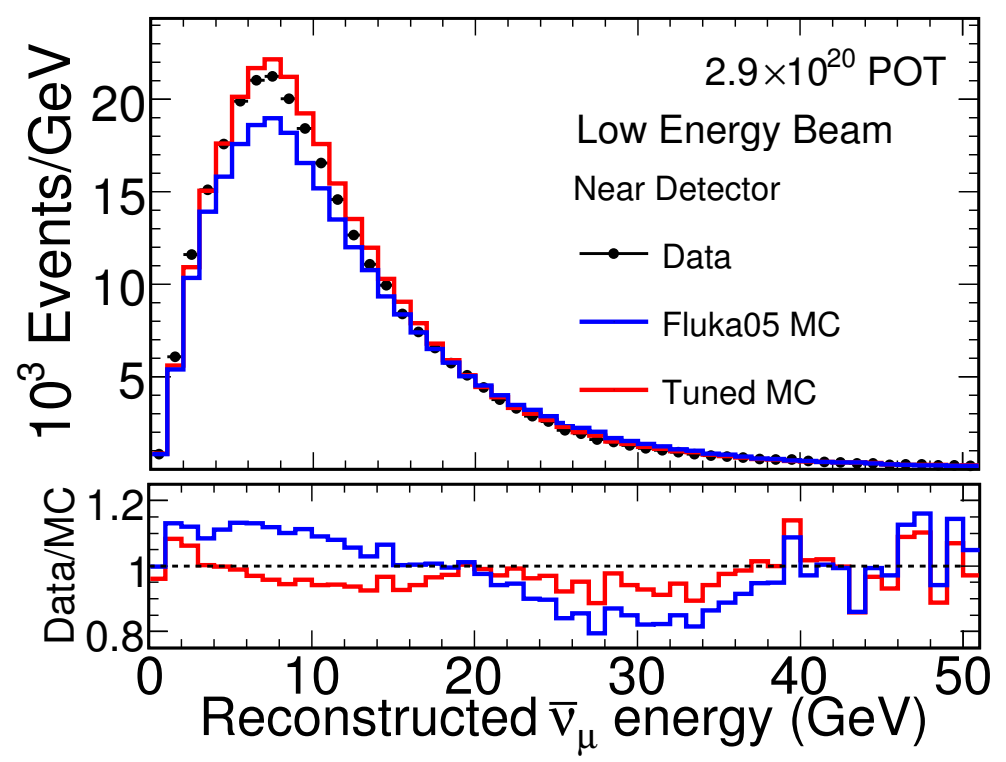

Figure 5.8: The selected $\bar{\nu}_{\mu}$ energy spectrum is shown for the ND; data (black points), untuned simulation (blue line), and simulation with tuned hadron production (red line). The ratios of the data to the two simulations are shown below this figure.

Fig. 5.9 shows the $\mathrm{X}$ and $\mathrm{Y}$ distributions of track vertices, along with the radius squared and $\mathrm{Z}$ distributions for the track vertices. The Monte Carlo is seen to model the data well.

\subsection{Extrapolation (the Beam Matrix)}

The two detector design of the MINOS experiment allows for reduction in the effect of a large number of systematic uncertainties. The systematics which affect both the detectors in the same way, such as the neutrino flux, the neutrino cross-section, and the modelling of the hadronic energy, cancel out to a large extent. But this cancellation would be exact only if the flux is same at both the Near and Far Detectors. For MINOS this is not true due to the decay kinematics of the parent particles that produce the neutrinos. The energy of the daughter neutrino for a given parent is given by:

$$
E_{\nu}^{*}=\frac{m_{p}^{2}-m_{\mu}^{2}}{2 m_{p}}
$$



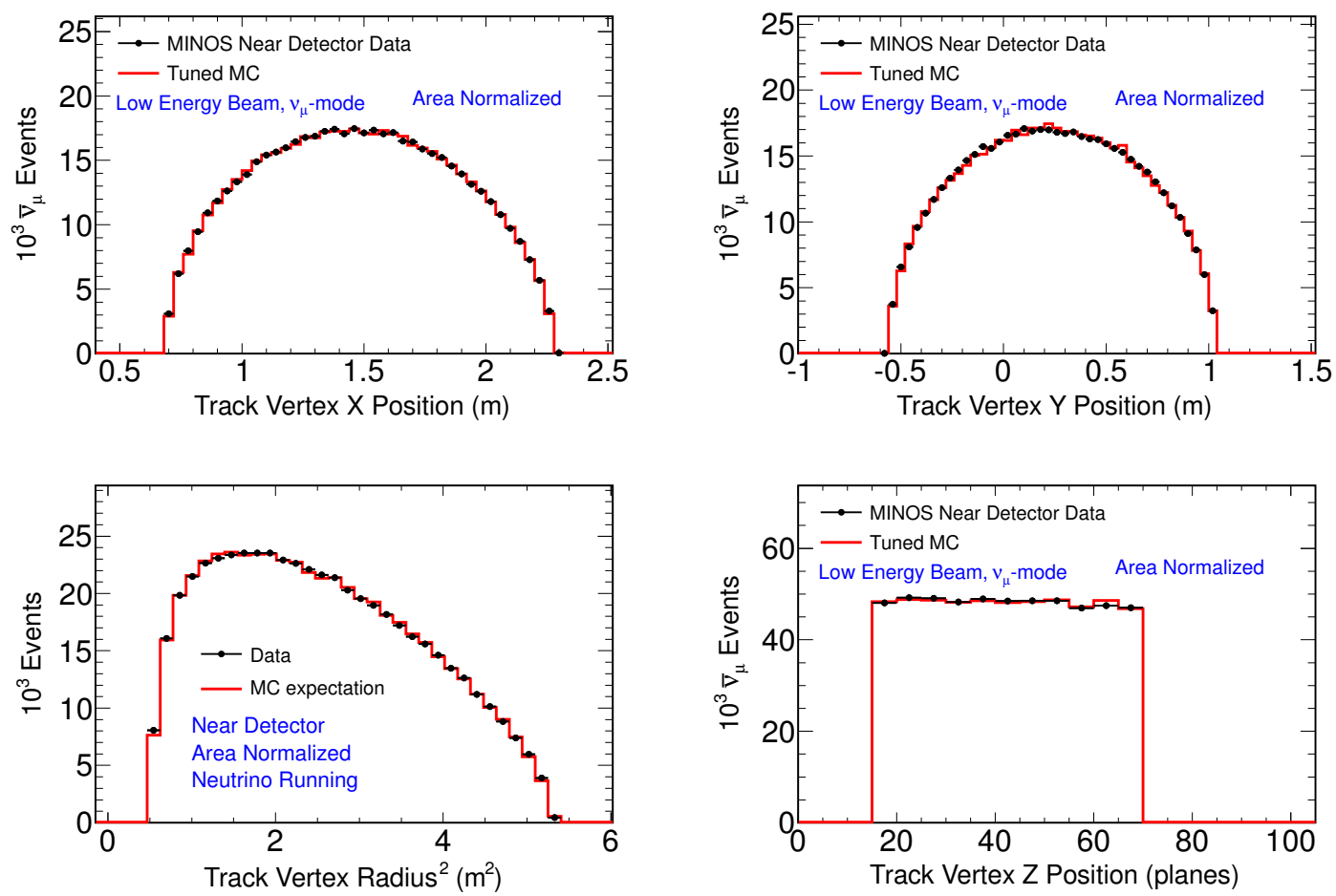

Figure 5.9: Top plots show the track vertex in $\mathrm{X}$ direction (left) and in $\mathrm{Y}$ direction (right) in meters. Bottom plots show the radius squared distribution of track vertex (left) and track vertex in $\mathrm{Z}$ direction (right) in units of number of planes. Black dots represent the ND data and the red curve represents Monte Carlo. The Monte Carlo is area normalised to data to compare the shapes of the distributions independent of normalisation.

where $m_{p}$ is the mass of the parent $\left(p=\pi^{ \pm}, K^{ \pm}\right), m_{\mu}$ is the mass of the muon and the neutrino mass is negligible. Thus the energy of the daughter neutrino in the parent's rest frame is fixed. However, in the lab frame the energy of the neutrino is given by:

$$
E_{\nu}=\frac{E_{\nu}^{*}}{\gamma_{p}\left(1-\beta_{p} \cos \theta\right)}
$$

where $\gamma_{p}$ is the parent's Lorentz factor and $\beta_{p}$ is its velocity. Therefore the neutrino energy in the lab frame depends on the relative angle, $\theta$, between the direction of travel of the parent and the neutrino.

The flux is also a function of angle. In the parent's rest frame the neutrinos are emitted 
isotropically, whereas, in the lab frame the flux becomes angle dependent:

$$
\frac{d N}{d \cos \theta}=\frac{1}{2 \gamma_{p}^{2}\left(1-\beta_{p} \cos \theta\right)^{2}}
$$

where again $\gamma_{p}$ is the parent's Lorentz factor and $\beta_{p}$ is its velocity.

Since the FD is $735 \mathrm{~km}$ away, for a given parent, a neutrino produced at only a very small range of angles w.r.t the initial beam direction will reach the FD. So we can assume that $\theta$ is unique for the neutrinos reaching the FD. Therefore, the energy of the neutrino at the FD will be uniquely determined by the parent energy. However the ND covers a wide solid angle since it is close to the decay pipe. Therefore the same parent can produce neutrinos at a large range of energies in the ND (Fig. 5.10). Consequently the spectrum at the ND and FD are slightly different as can be seen in Fig. 5.11. The parents responsible for producing neutrinos in the coloured areas in the ND bins produce a different neutrino energy distribution in the FD bins with the corresponding colours. This effect is more pronounced for parents with higher energy which travel further down the decay pipe before decaying. This enhances the solid-angle effect and allows a wider range of contributing decay angles at the ND (this outweighs the increased Lorentz boost whose effect is to narrow the outgoing neutrino energy distribution). Thus the neutrino energy at the ND is lower as compared to that at the FD, shifting the neutrino events at the ND downward into the peak. The net effect is that the ND spectrum is more peaked as compared to the FD spectrum.

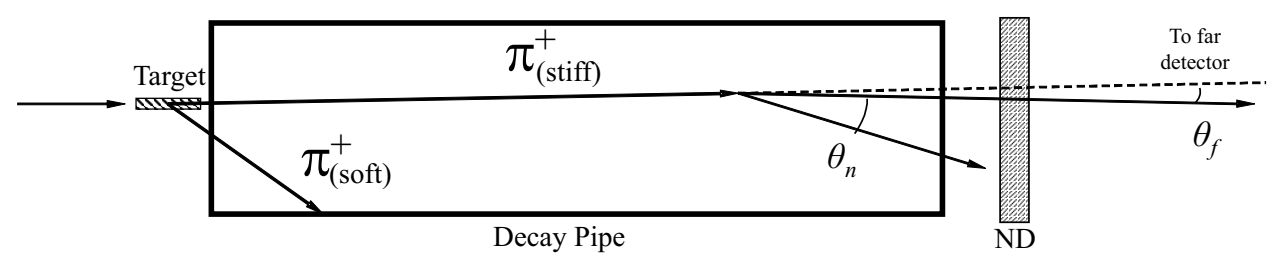

Figure 5.10: Diagram of the neutrino parents in the NuMI decay pipe. A parent will typically have a wide range of neutrino decay angles that reach the Near Detector and a very narrow range that will reach the Far Detector.

To predict the FD spectrum, given the ND spectrum, a method called "beam matrix" 

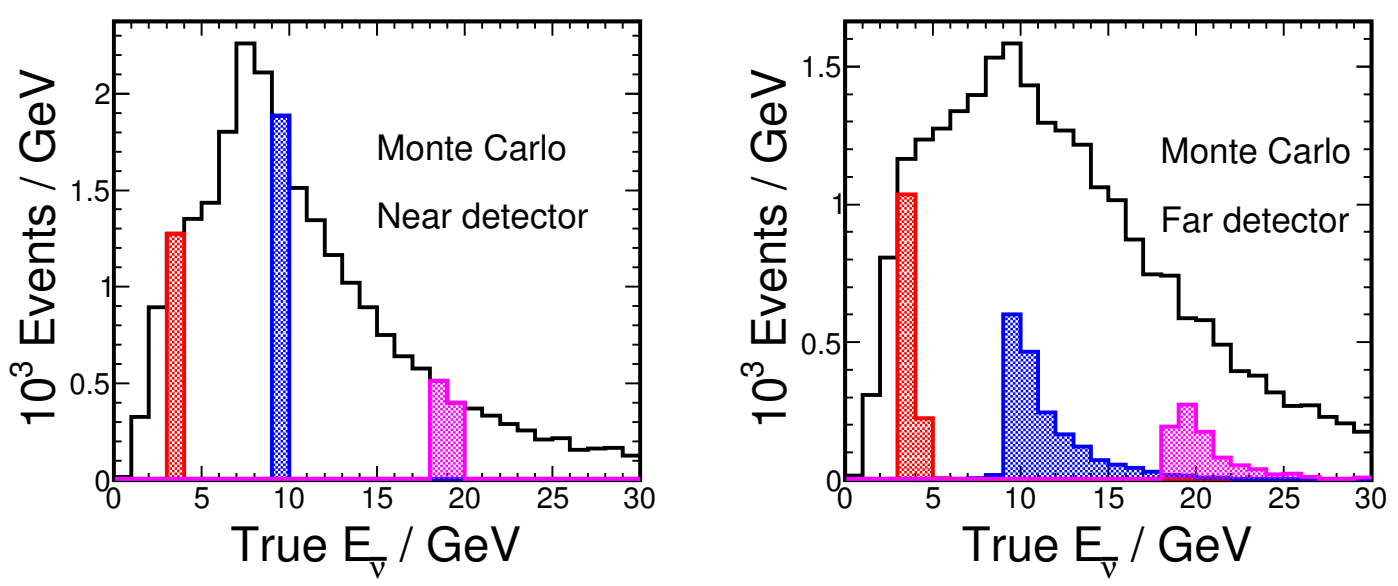

Figure 5.11: Simulated true neutrino energy spectra in the near (left) and and far (right) detectors. The coloured regions on the left and right spectra show the neutrino energy distributions coming from the same neutrino parents.

extrapolation is adapted for antineutrinos is used [136]. The Monte Carlo is used to create a matrix, called the "beam matrix", that relates the ND energy spectrum to that at the FD. The rows of the beam matrix correspond to a bin of FD true neutrino energy and the columns each correspond to a bin of ND true neutrino energy. Simulated neutrino parents are allowed to decay in the ND and FD, at randomly selected interaction vertices. The neutrino energy and the probabilities of those decay directions are then determined by the decay kinematics. The neutrino energy at the ND can then be associated with that at the FD via the shared parent. We get the complete beam matrix by repeating the procedure for all the simulated parents. This procedure is carried out for each run period separately to take into account differences in the beam properties, and also independently for neutrinos and antineutrinos to account for the different parent particles. Since the matrix is nearly diagonal it shows that there is a strong correlation between neutrino energies at the Near and Far Detectors, but each near detector energy corresponds to a distribution of far detector energies. The matrix for antineutrinos in the FHC beam can be seen in 5.12

Since this matrix is based on simulation it assumes that the Near and Far Detector spectra are in true energy and are perfectly pure. Moreover, it assumes that the events 


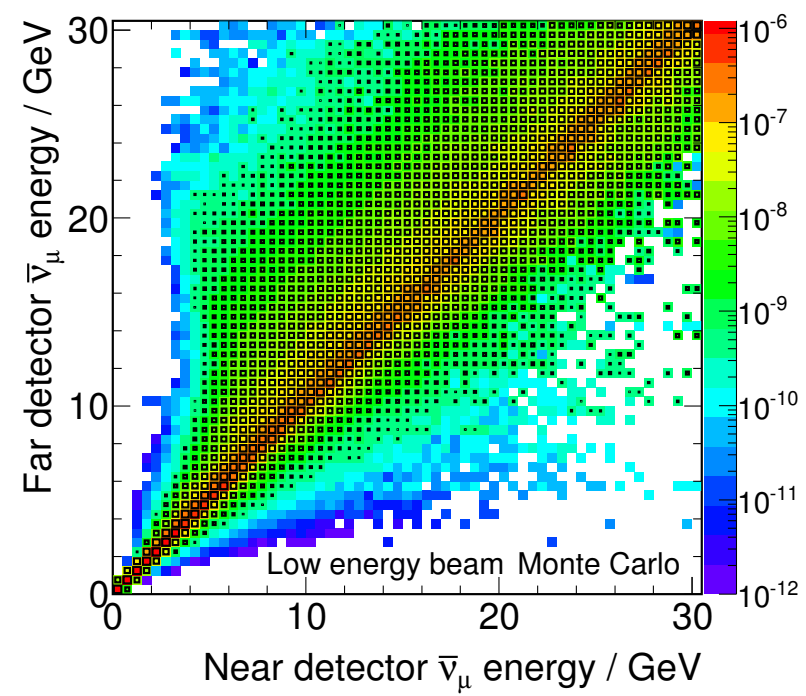

Figure 5.12: The beam matrix for $\bar{\nu}_{\mu}$ 's in the FHC beam. Each cell relates a FD energy bin to a ND one. The content of each cell represents the mean number of $\bar{\nu}_{\mu}$ events expected in the FD for one event in the ND. This distribution is treated as a matrix to relate the energies measured in the ND to those expected in the FD.

are selected perfectly. But in reality, the selection efficiency is not perfect, and there is background contamination. Also, the beam matrix transforms the ND flux in true energy to the FD flux. But the Near and Far Detectors do not directly measure neutrino flux, but a visible energy distribution. Therefore, additional steps are required to complete the extrapolation procedure. Additional matrices, derived from Monte Carlo simulations, are used to remove neutral-current contamination from the observed near detector spectrum. The observed reconstructed spectrum at the ND is converted to true energy, and corrected for purity, reconstruction efficiency, and selection efficiency to get the ND flux. The ND flux is extrapolated to the FD by using the beam matrix. The equivalent matrices are then applied at the FD in reverse to generate the FD prediction in reconstructed energy. The flowchart in Fig. 5.13 illustrates the procedure for neutrino analysis. The procedure is adapted for antineutrino analysis, shown in Fig. 5.14, where the $\nu_{\mu}$ and $\bar{\nu}_{\mu}$ spectra are separated. The $\nu_{\mu}$ background in the $\bar{\nu}_{\mu}$ event selection is oscillated according to the $\nu_{\mu}$ oscillation parameters. Similarly, the $\bar{\nu}_{\mu}$ background in the $\nu_{\mu}$ event selection is oscillated 


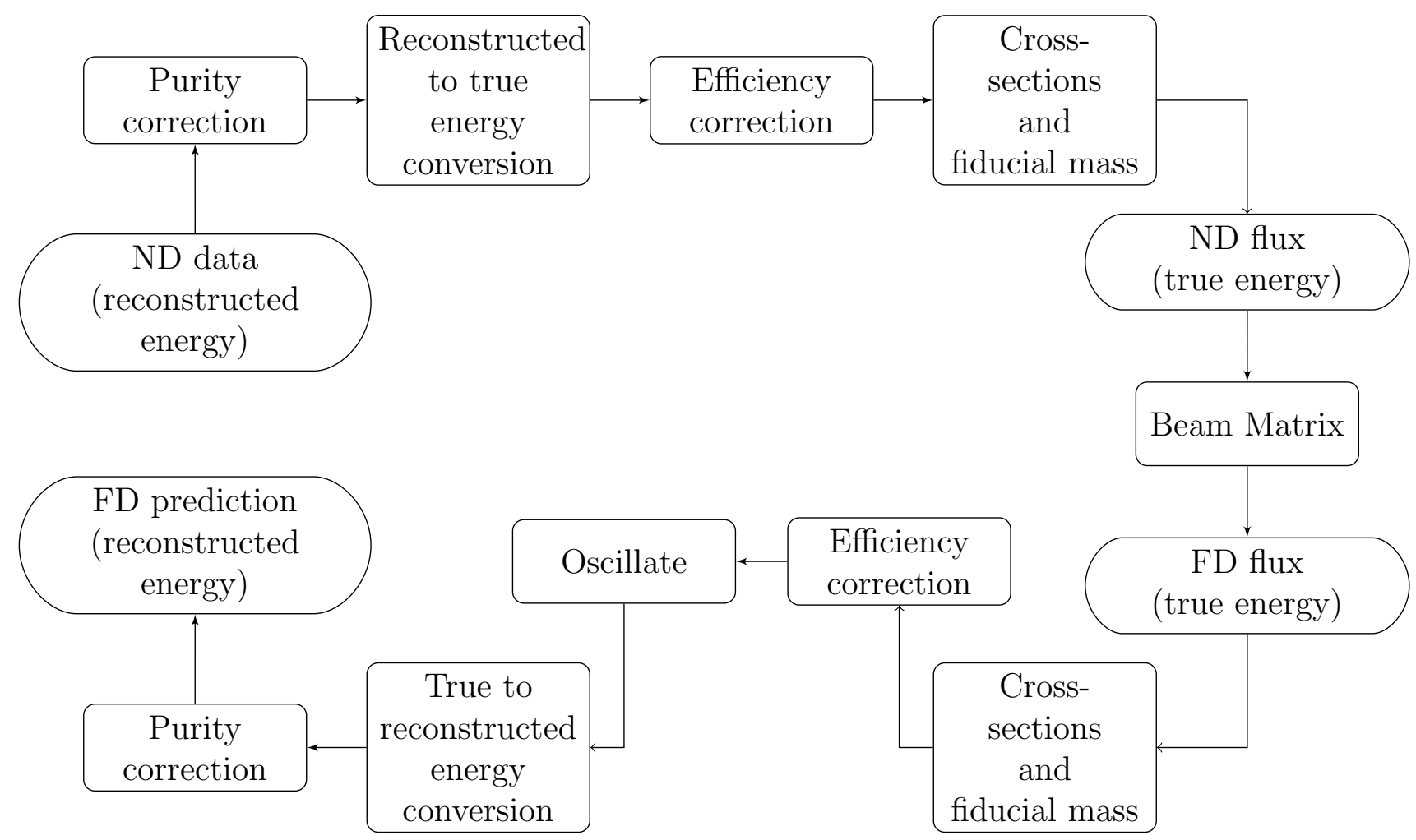

Figure 5.13: Flowchart showing the steps involved in the extrapolation of a Near Detector energy spectrum to a Far Detector prediction.

according to the $\bar{\nu}_{\mu}$ oscillation parameters in order to make their correct prediction. The $\nu_{\mu}$ and $\bar{\nu}_{\mu}$ spectra are extrapolated individually, and the $\nu_{\mu}$ prediction is used to calculate the CC background to the FD $\bar{\nu}_{\mu}$ spectrum, and vice-versa. The FD prediction can be produced for any choice of oscillation parameters by applying the oscillation probability to the true FD flux times cross-section before applying the other corrections.

We can confirm that the extrapolation is behaving as expected by making a FD prediction using ND Monte Carlo, with the oscillation parameters set to zero. If everything is working properly, this should produce a FD spectrum that is nearly identical to the unoscillated FD Monte Carlo. But we should not expect the two to be exactly identical since the ND and FD Monte Carlo are statistically independent. The ratio of the FD Monte Carlo to the FD prediction using the ND Monte Carlo can be seen in Fig. 5.15, and the fluctuation is seen to be $\sim 3 \%$ level at all energies. Since the fluctuations are smaller than the FD data, so the agreement is considered to be sufficient to the analysis. 


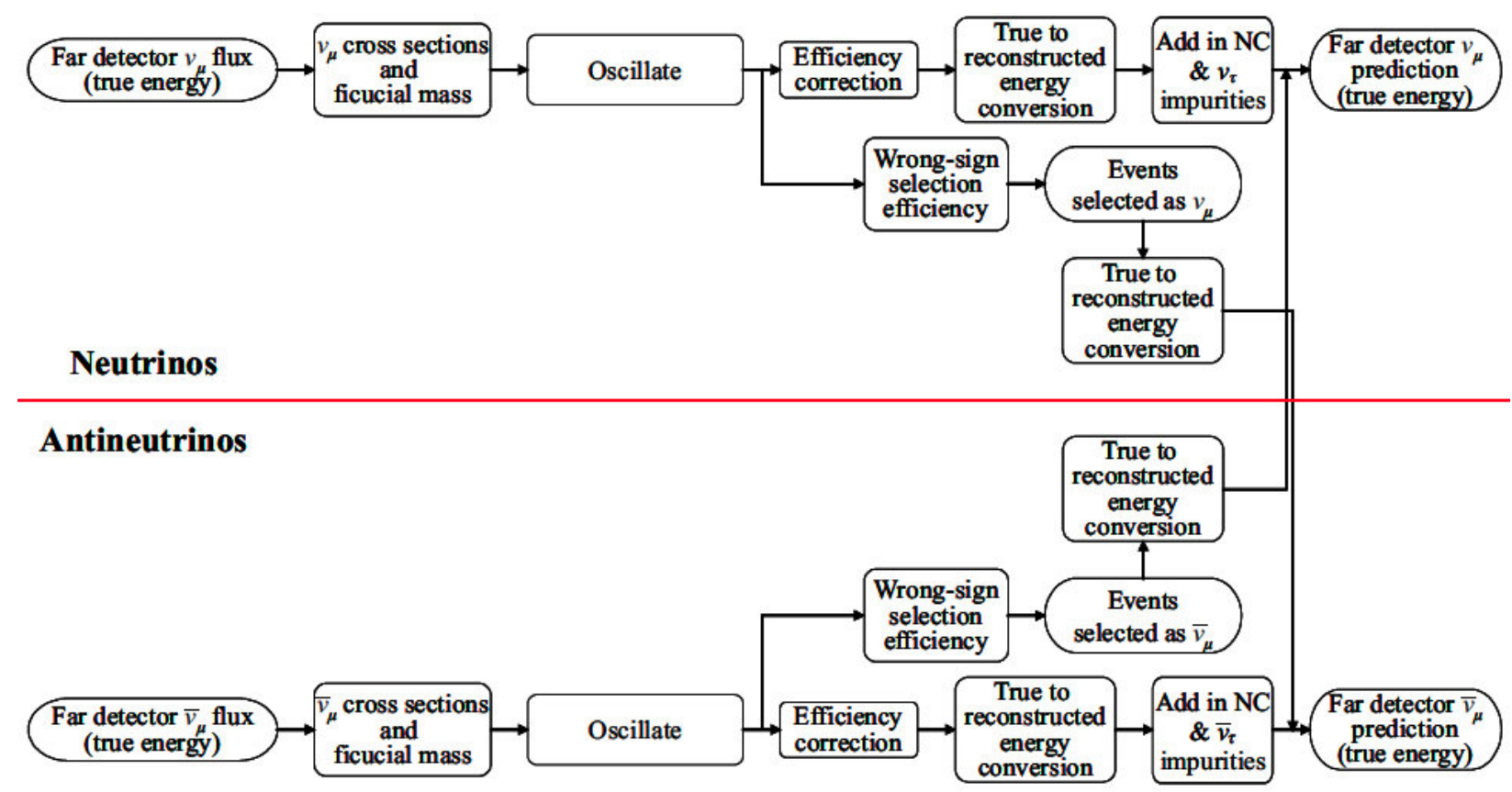

Figure 5.14: Beam matrix extrapolation procedure for antineutrinos, starting from the Far Detector flux calculated using Fig. 5.13. Figure from [136].
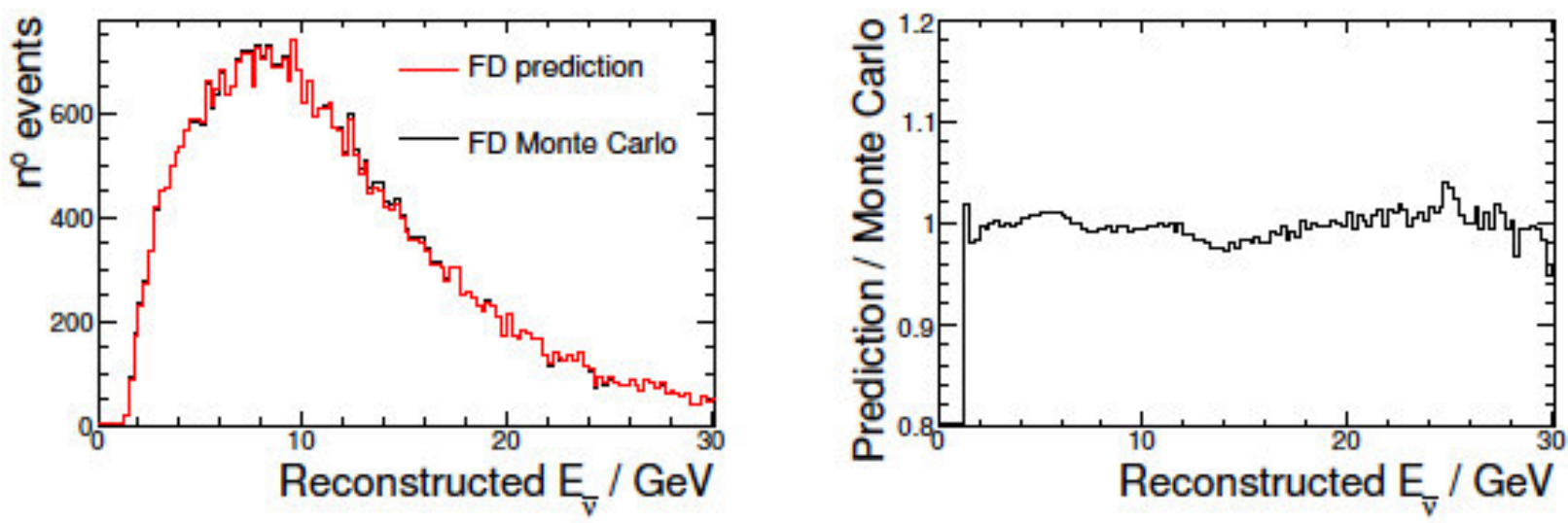

Figure 5.15: Left: the raw FD Monte Carlo (black) and the prediction obtained by applying beam matrix extrapolation to the ND Monte Carlo (red) for the $\mathrm{CC} \bar{\nu}_{\mu}$ spectra. Right: the corresponding ratios of the prediction divided by the FD Monte Carlo.

\subsection{Far Detector Data}

The FD data sample is selected using the same selection variables optimised for the ND data. In addition, in order to remove the contamination from cosmic muons, tracks are required to have at most an angle $\theta$ with the beam axis such that $\cos \theta<0.6$. The overall 
efficiency of this selector at the FD is shown in Fig. 5.16. The analysis is done as a blind analysis, which means that we don't look at the FD data until the entire analysis chain is finalised. It is still necessary to check that the data are well modelled by the Monte Carlo simulation. To do this we look at the FD distribution of variables that are insensitive to oscillations, such as spatial distributions. The overall normalisation of the event count is hidden. In order to study the oscillation-sensitive distributions, such as energy spectra, a blinding algorithm is applied to the data which removes a subset of the events from the dataset in a way that will mask the effect of oscillations. After the analysis is finalised the data is unblinded and the proper normalised versions of these comparisons are performed with the full dataset. Fig. 5.17, 5.18, and 5.19 show the data/MC comparison of the CC/NC

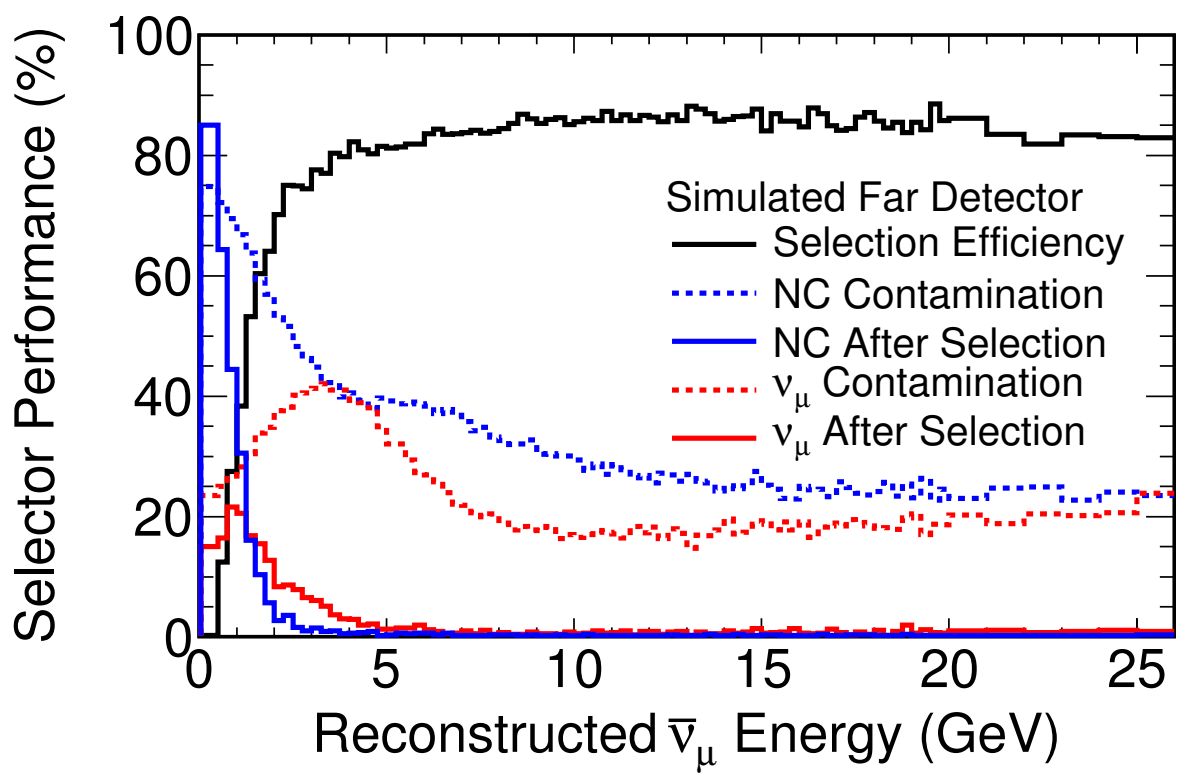

Figure 5.16: Efficiency and Purity at the FD before and after selection cuts.

separation variable, the charge sign selections at the far detector. Fig. 5.20 and 5.21 show the spatial distributions of track vertices and ends in the detector. The simulation with no-oscillations is shown in black, that with the same oscillations as neutrinos is shown in blue, and the data points are shown in black. All these distributions show that the data is very well modelled by simulation. 


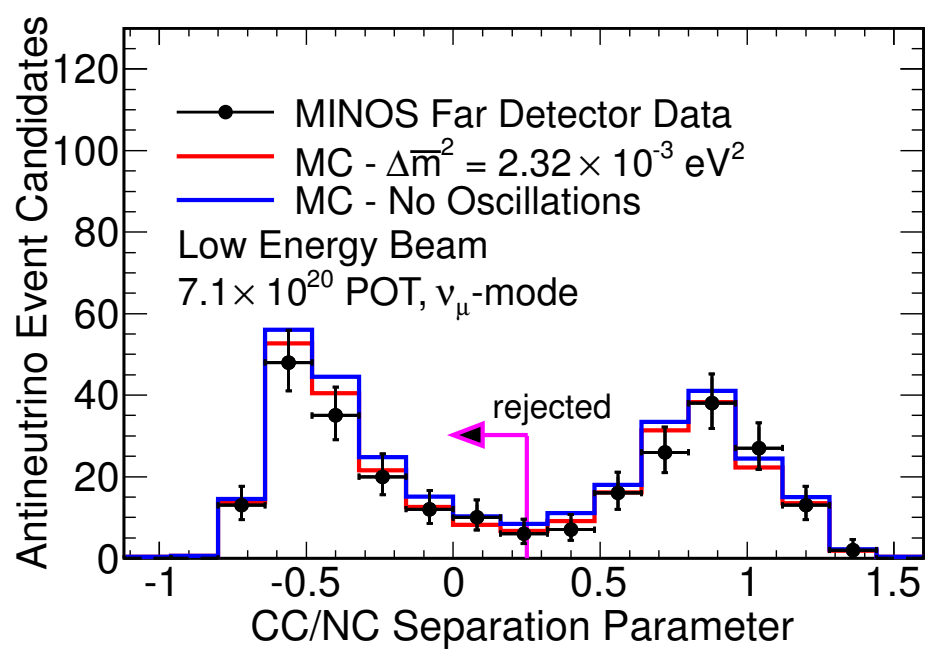

Figure 5.17: CC/NC separation parameter $(D p I D)$ is shown for the FD; data (black points), oscillated simulation (solid red line) and unoscillated simulation (dashed red line). All other selection cuts have been applied.

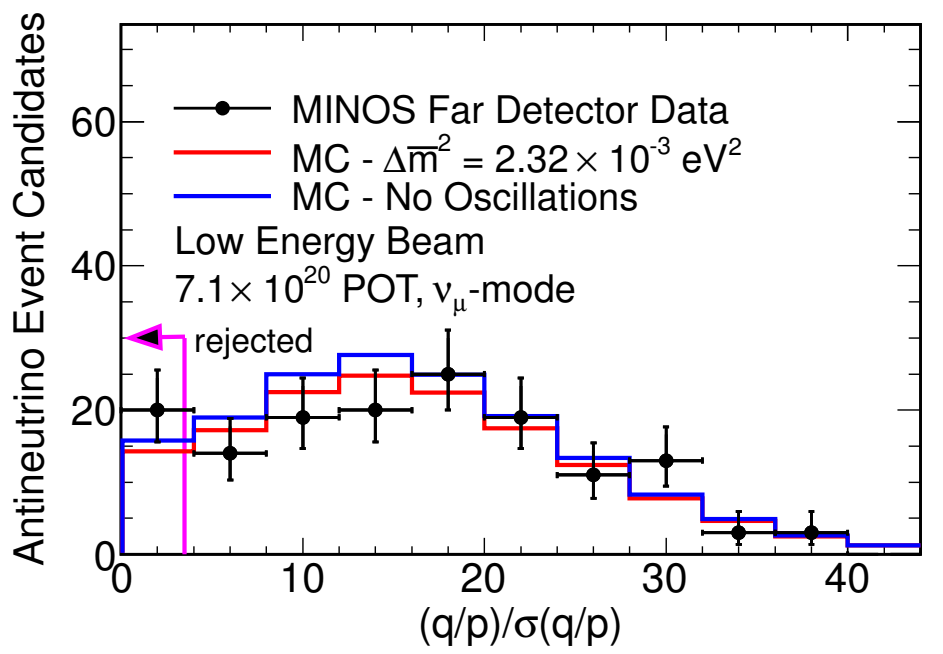

Figure 5.18: Charge-sign selection variable $\frac{\frac{q}{p}}{\sigma\left(\frac{q}{p}\right)}$ is shown for the FD; data (black points), oscillated simulation (solid red line) and unoscillated simulation (dashed red line). All other selection cuts have been applied.

\subsection{Systematics}

A number of systematic uncertainties which can affect the oscillation measurements [137] are described below. 


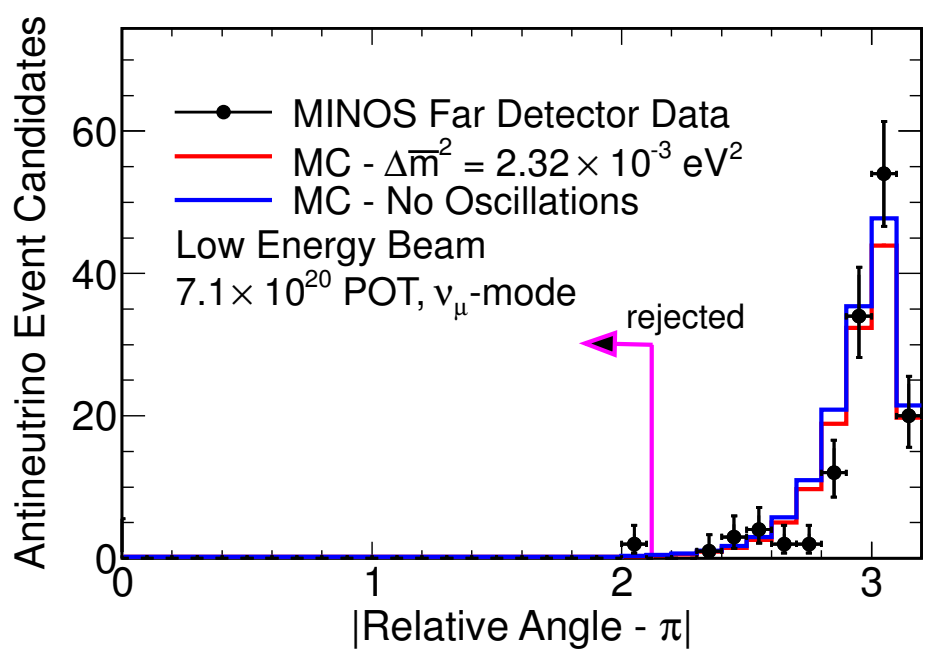

Figure 5.19: Charge-sign selection variable - RelativeAngle- $\pi$ - is shown for the FD; data (black points), oscillated simulation (solid red line) and unoscillated simulation (dashed red line). All other selection cuts have been applied.
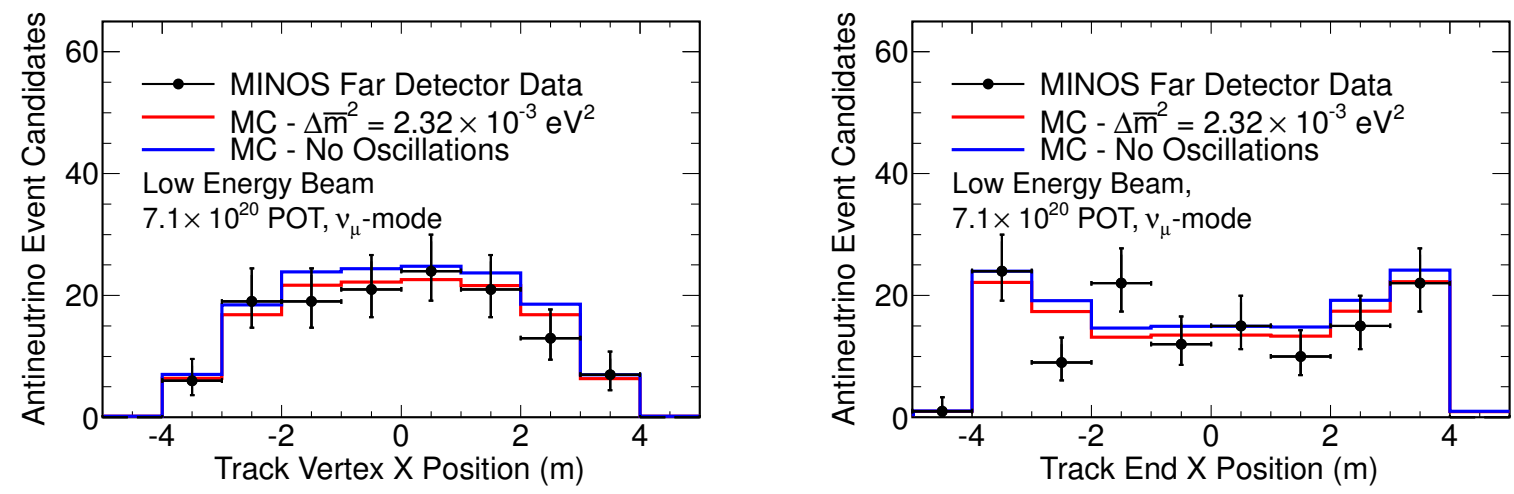

Figure 5.20: Selected antineutrino event vertex (left) and end (right) positions for the FD as a function of $x$-coordinates.

\subsubsection{Track Energy Scale}

There is a systematic uncertainty of $2 \%$ on muon track energy from range. It is measured using the calibration detector. The uncertainty on the track energy from curvature is $4 \%$. It is determined by comparing range and curvature momentum measurements for stopping tracks. These uncertainties are taken as fully correlated between the Near and Far Detectors. 

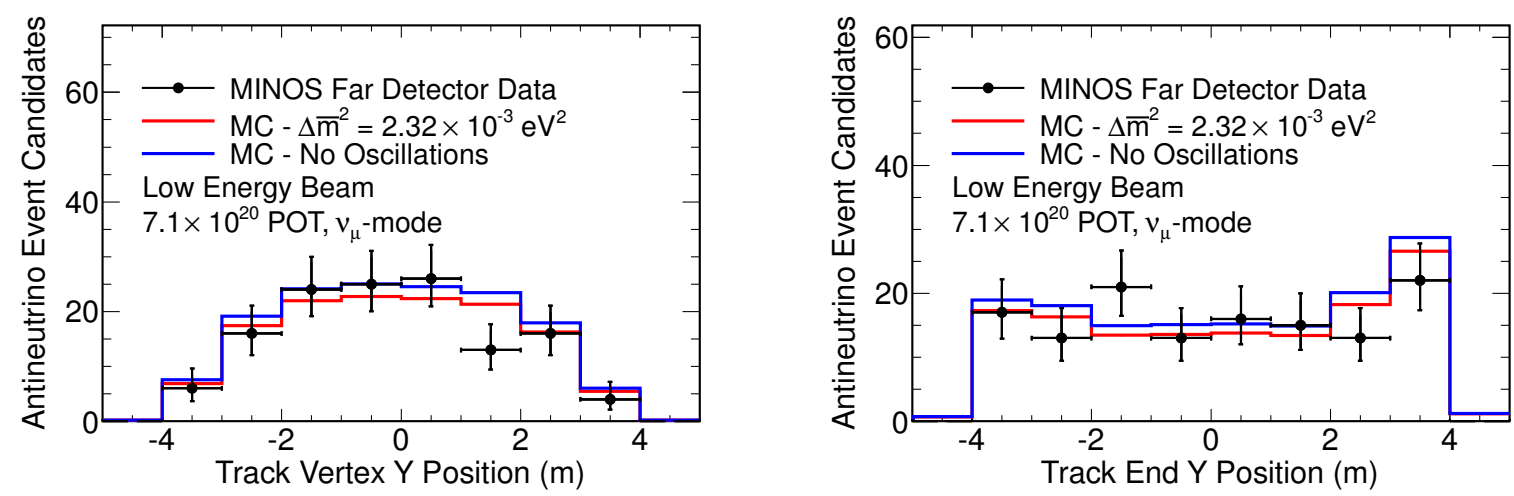

Figure 5.21: Selected antineutrino event vertex (left) and end (right) positions in the FD as a function of $y$-coordinates.

\subsubsection{Shower Energy Scale}

It has two components: uncertainty in the relative shower energy scale and absolute shower energy scale. The relative shower energy systematics come from uncertainties in the energy calibration at the near $(1.9 \%)$ and far $(1.1 \%)$ detectors and are uncorrelated between the detectors. The absolute shower energy systematic uncertainty is taken as fully correlated between the two detectors and has two major components. The first component stems from uncertainties in the detector response to single hadrons as measured in the calibration detector at CERN test beam and is $5.7 \%$ at all energies. The second component is energy-dependent and encapsulates uncertainties in hadron production and intranuclear effects. It is $8.2 \%$ at the lowest energies and decreases to $3 \%$ above $10 \mathrm{GeV}$. The final systematic has the energy-dependent form

$$
\sigma_{\mathrm{shw}}=6.6 \%+(3.5 \%) \times e^{\frac{-E \mathrm{shw}}{1.44 \mathrm{GeV}}}
$$

which is taken as fully correlated between the two detectors. 


\subsubsection{Backgrounds}

The uncertainty in the NC background is primarily due to mis-modelling of hadronic showers and neutral-current cross sections in the Monte Carlo. Misidentified $\nu_{\mu} \mathrm{CC}$ events also make up a significant component in the selected $\bar{\nu}_{\mu} \mathrm{CC}$ dataset. It is due to the misidentification of the charge of the muons forming the muon track. The systematic uncertainty on these backgrounds is quantified by studying the subsets of data and Monte Carlo in the ND where the number of misidentified events has been enhanced by selecting events with $0<D p I D<0.25$. The data and Monte Carlo in the enriched region are compared, and any discrepancy in the oscillation region is attributed to the wrong-sign background. The maximum size of this discrepancy is taken as the uncertainty on this background. This gives a systematic uncertainty of $50 \%$ on the $\mathrm{NC}$ and wrong sign CC backgrounds.

\subsubsection{Near to Far Normalisation}

The normalisation systematic is $4 \%$ for $\bar{\nu}_{\mu}$ sample. It incorporates several systematic uncertainties, all of which change the relative number of events expected at the two detectors per POT. The $4 \%$ systematic for the $\bar{\nu}_{\mu}$ sample is dominated by a $3 \%$ uncertainty on the difference in reconstruction and selection efficiency in the two detectors. Table 5.1 below shows all the contributions. The 'fiducial bias' uncertainties refer to data-simulation differences in the non-uniformity of the vertex distributions coming from acceptance effects due to the geometry of the ND.

\subsubsection{Cross-sections}

Several systematic effects are evaluated as part of the cross-section systematic, both on the overall cross-section and on various NEUGEN [129] interaction model parameters. Some affect both neutrinos and antineutrinos and others are specific to antineutrinos. While the majority of the cross-section uncertainties cancels between the two detectors, some residual systematic effect remains because of the spectral differences between the detectors. 


\begin{tabular}{cc}
\hline Systematic & Uncertainty \\
\hline Steel Thickness & $0.2 \%$ \\
Scintillator Thickness & $0.2 \%$ \\
FD Live Time & $1.0 \%$ \\
ND Fiducial Bias (z) & $1.9 \%$ \\
ND Fiducial Bias (y) & $0.7 \%$ \\
ND Fiducial Bias (x) & $0.7 \%$ \\
N/F Selection Bias & $3.0 \%$ \\
\hline
\end{tabular}

Table 5.1: Components of the near-to-far normalisation systematic uncertainty. Table from 138

\subsubsection{Flux Modelling}

The flux modelling uncertainty arises from a number of sources of errors, including hadron production, beam optics (horn positions, currents, etc.), the position of the target, the shape and the amount of material in the horns and the beamline. The flux errors are evaluated by changing the fit parameters in the beam tuning fit within their uncertainties and observing the effect on the flux. The majority of the flux modelling errors cancel between the two detectors, but some uncertainty still remains because the two detectors do not see identical fluxes. However it has very little impact on the final oscillation analysis.

\subsubsection{Downstream Events}

Downstream production, or decay pipe production, refers to the antineutrinos that come from the decay of hadrons coming from the protons interacting outside the target. The interaction of protons outside the target is primarily in the decay pipe walls, but a smaller component also comes from the interaction in the concrete surrounding the decay pipe or the helium in the decay pipe. The parents produced in the downstream region give rise to $11 \%$ of the ND $\bar{\nu}_{\mu}$ events and $7 \%$ of the $\mathrm{FD} \bar{\nu}_{\mu}$ events. The uncertainty on this systematic is significant for the antineutrino analysis because it does not produce the same spectrum in the Near and Far Detectors, so errors in production cross-sections do not cancel between the two detectors. In order to constrain the uncertainty on this systematic the ND data is 
used. The ND Monte Carlo is first reweighted using a special set of beam weights, called SKZP weights, obtained from beam fits. Then the decay pipe component is scaled up or down in order to make the total number of Monte Carlo events less than $20 \mathrm{GeV}$ match the data. Using this method a 'worst case' uncertainty of $\pm 100 \%$ is obtained on the number of neutrinos coming from interactions outside of the target.

\subsubsection{Effect on the Analyses}

The effect of each systematic uncertainty on the oscillation results is estimated using the Monte Carlo. Systematic shifts are applied to high statistics ND and FD Monte Carlo spectra to obtain shifted spectra. Positive as well as negative values of the shifts are applied, producing two sets of spectra. The shifted ND spectra are used to make shifted FD prediction for each systematic. The ratio of the shifted spectrum to the unshifted spectrum gives us the size of the uncertainty of a systematic. The systematic error band is constructed by calculating the quantity

$$
1-\frac{\text { Shifted FD Prediction/Shifted FD MC }}{\text { Nominal FD Prediction/Nominal FD MC }}
$$

for each systematic. Positive values of this quantity are added in quadrature in each energy bin to obtain the upper band, and negative values are added to obtain the lower band. Fig. 5.22 shows the FD systematic error band constructed from all systematic uncertainties summed in quadrature. This error band is not used in the fit but is used in the plots comparing our prediction and data to show the systematic uncertainty on the FD prediction.

\subsection{Oscillation Analysis}

The oscillation parameters are measured using a two-parameter fit for $\Delta \bar{m}_{32}^{2}$ and $\sin ^{2} 2 \bar{\theta}_{23}$ from Eq.5.1. The oscillation parameters are varied within a certain range and a FD prediction is made for each value of the parameter. The fit finds the oscillation parameters that 


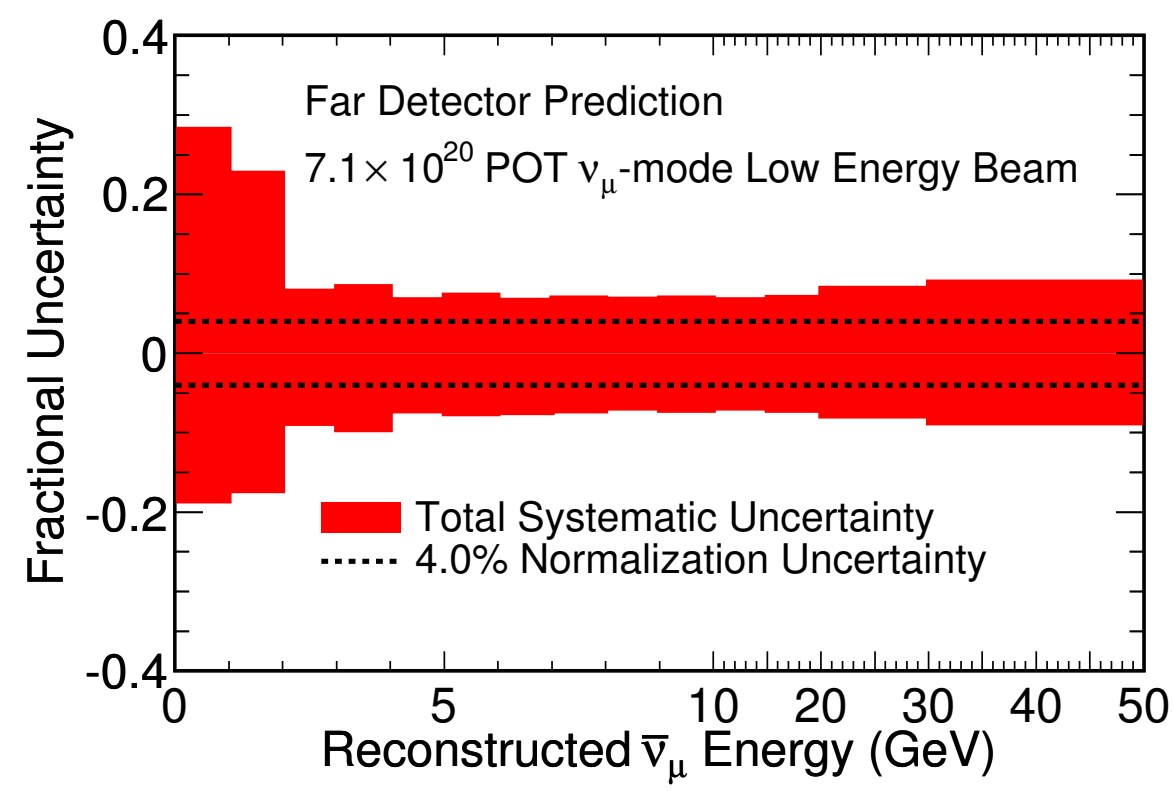

Figure 5.22: FD systematic error band constructed from all systematic uncertainties summed in quadratue.

maximise the likelihood or minimise the $-2 \ln L$ of the data given the prediction at those oscillation parameters:

$$
-2 \ln L(\mathbf{W})=2 \sum_{i}\left[p_{i}(\mathbf{W})-d_{i}+d_{i} \ln \frac{d_{i}}{p_{i}(\mathbf{W})}\right]
$$

where $\mathbf{W}=\left(\Delta \bar{m}_{32}^{2}, \sin ^{2} 2 \bar{\theta}_{23}\right)$ contains the fit parameters, $i$ is the energy bin, $d_{i}$ is the number of data events in bin $i$, and $p_{i}(\mathbf{W})$ is the predicted number of events in bin $i$ given the parameters in $\mathbf{W}$. The prediction, $p_{i}$, is generated based on the ND data as described in Section 5.3. The oscillation formula (5.1) is applied to the predicted spectrum in true energy before it is transformed into reconstructed energy for comparison with the data. Once a likelihood surface has been produced, the contours are drawn using the FeldmanCousins method [139, 140]. Using this method helps address three difficulties [128]. First, the effects being studied exist near physical boundaries where gaussian confidence intervals cover more than the expected fraction of experiments for a given confidence level. The 
boundaries exist at $\sin ^{2} 2 \bar{\theta}_{23}=0,1$ and $\Delta \bar{m}_{\text {atm }}^{2}=0$. Second, when the statistics in the spectrum are low, the oscillation formula has a degeneracy between oscillations with a high $\Delta \bar{m}_{\text {atm }}^{2}$ and $\sin ^{2} 2 \bar{\theta}_{23} \approx 1$, and a low $\Delta \bar{m}_{\text {atm }}^{2}$ with $\sin ^{2} 2 \bar{\theta}_{23}=0.5$. This degeneracy creates the opposite effect as the physical boundary: the gaussian confidence intervals do not cover enough experiments. Third, the Feldman-Cousins method allows us to introduce the effects of systematics into our confidence intervals and contours.

Fig. 5.23 shows the FD data spectrum along with the prediction based on ND data without oscillations, with the same oscillation parameters as the neutrino, and for the background. A total of 130 events are observed with an unoscillated expectation of 150 and an oscillated expectation of 136 events assuming the same oscillation parameters as measured for neutrinos in [141].

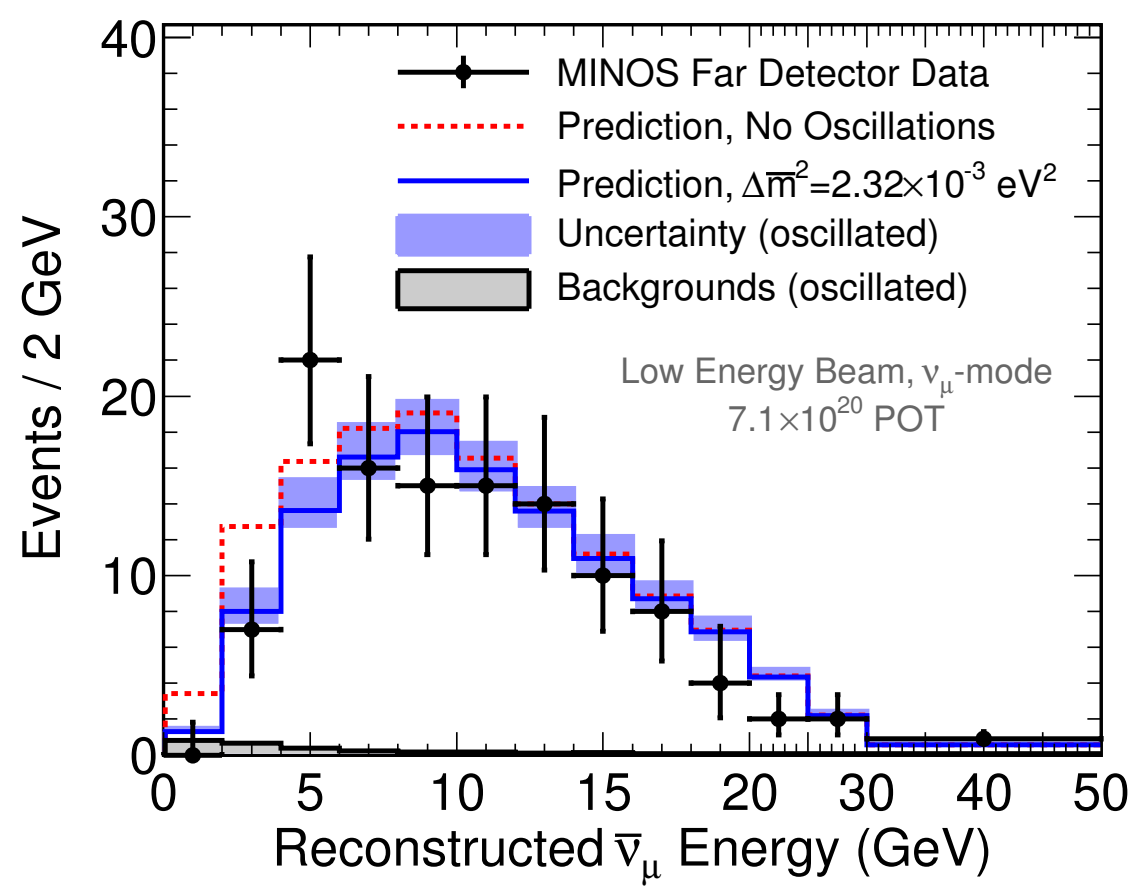

Figure 5.23: Far Detector $\bar{\nu}_{\mu}$ data spectrum (dots) compared to predictions with no oscillations (dashed red histogram) and with best-fit oscillation parameters. Error bars on the data points represent the statistical errors and the band around of the histogram with bestfit prediction represents the total systematic uncertainty. Total background in the best-fit prediction is also displayed (gray shaded histogram). 
The allowed oscillation contours are shown in Fig. 5.24. The best fit is at a relatively high mass splitting since the spectral deficit is at high energy, and even higher mass splitting cannot be excluded. Much of the power of the analysis comes from counting events, rather than shape information, due to its low statistics. Consequently, the results cannot distinguish the best fit point with a moderate mixing angle from fast oscillations with a large mixing angle. Significant power can be gained by assuming maximal mixing and looking in only the mass splitting parameter. The one-dimensional exclusion curve is shown on the right in Fig. 5.24. We exclude $\Delta \bar{m}^{2}>4.49 \times 10^{-3} \mathrm{eV}^{2}$ at a $3 \sigma$ significance. This data helps provide additional bounds on the value of $\Delta \bar{m}^{2}$ in addition to the constraints provided by the analysis of antineutrino data obtained in a dedicated antineutrino running [142]. For comparison we also show the $90 \%$ confidence level contour for the MINOS neutrino oscillation parameters, from [141]. 

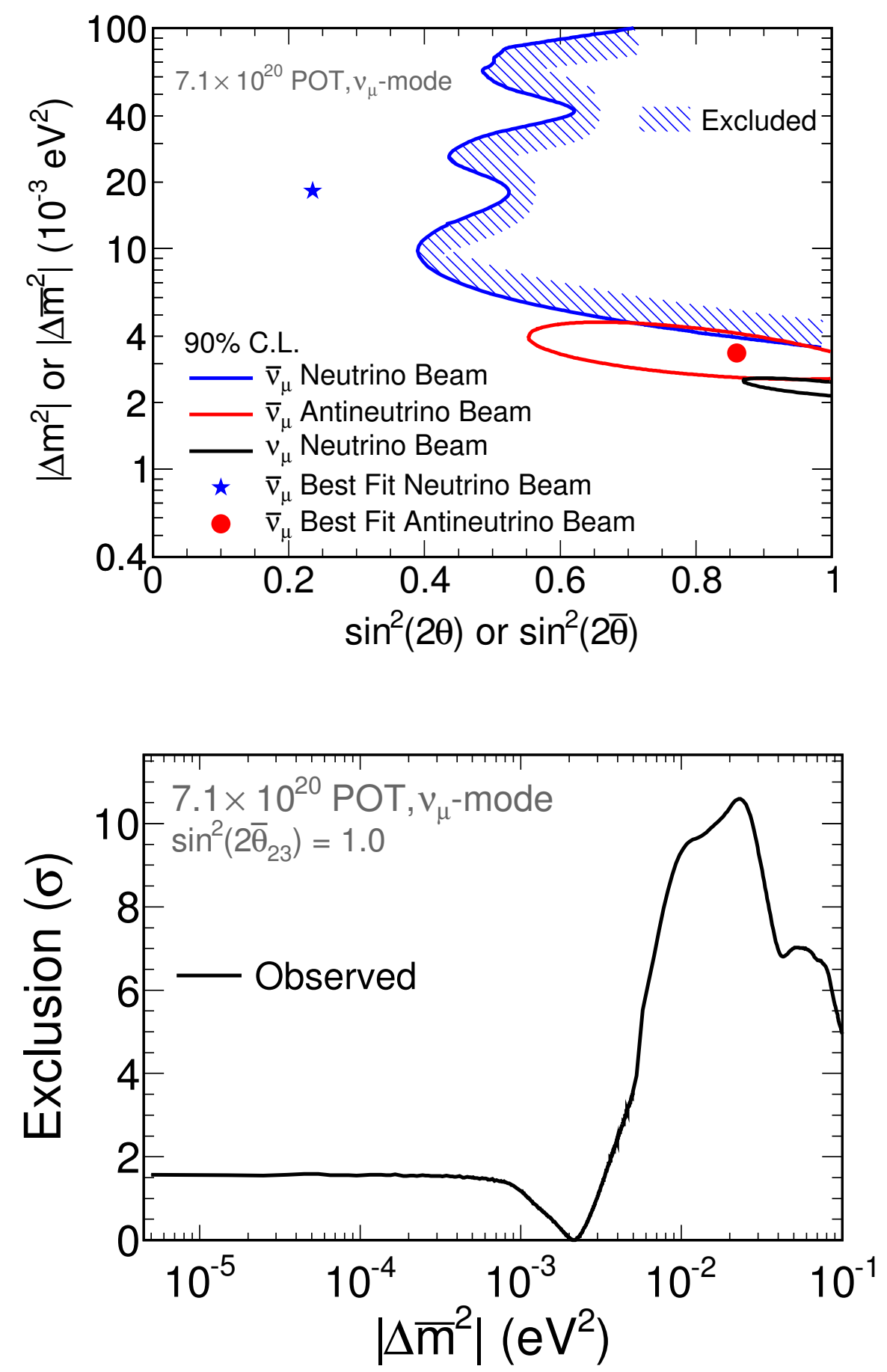

Figure 5.24: (Top) The 90\% antineutrino oscillation contours from FHC running, overlaid with the $2010 \mathrm{CC} \nu_{\mu}$ result and the $2010 \mathrm{RHC} \bar{\nu}_{\mu}$ result. The contours are determined using the Feldman-Cousins method. The best fit is at $\left|\Delta \bar{m}_{\text {atm }}^{2}\right|=18 \times 10^{-3} \mathrm{eV}^{2}$ and $\sin ^{2}\left(2 \bar{\theta}_{23}\right)=$ 0.25. The hatched area indicates the excluded region. (Bottom) One-dimensional fit of $\Delta \bar{m}^{2}$ at maximal mixing, showing the values excluded by this result. At maximal mixing, we exclude $\Delta \bar{m}^{2}>4.49 \times 10^{-3} \mathrm{eV}^{2}$ at a $3 \sigma$ significance. 


\section{Chapter 6}

\section{Neutrino to Antineutrino Oscillations}

As discussed in Chapter 2, the violation of Lorentz and $\mathcal{C P} \mathcal{T}$ symmetries, described by SME, can lead to mixing between neutrinos and antineutrinos. The violation of these symmetries is expected to be very small since these are suppressed by the Planck scale. But the interferometric nature of neutrino oscillations enhances the effect of these violations large enough to be observed in a long-baseline experiment. The MINOS experiment has the unique ability to test these oscillations because it is a magnetised detector (Section 3.4) and, therefore, can distinguish between neutrinos and antineutrinos by charge-sign identification of muons resulting from their charged-current interactions, (Section 4.2.1). Considering a subset of SME coefficients which cause time-independent oscillations the oscillation probabilities were derived in Chapter 2, They are:

Survival probability of $\nu_{\mu}$ :

$$
P\left(\nu_{\mu} \rightarrow \nu_{\mu}\right)=1-\left(\sin ^{2}\left(\theta_{C \text {-odd }}-\theta_{C \text {-even }}\right)+\frac{1}{4}\left[\sin \left(2 \theta_{C \text {-odd }}\right)+\sin \left(2 \theta_{C \text {-even }}\right)\right]^{2}\right) \sin ^{2}\left(\frac{\Delta m^{2} L}{4 E}\right)
$$




\section{Oscillation probability $\nu_{\mu}$ to $\bar{\nu}_{\mu}$ :}

$$
P\left(\nu_{\mu} \rightarrow \bar{\nu}_{\mu}\right)=\left(\sin ^{2}\left(\theta_{C \text {-odd }}-\theta_{C \text {-even }}\right)-\frac{1}{4}\left[\sin \left(2 \theta_{C \text {-odd }}\right)-\sin \left(2 \theta_{C \text {-even }}\right)\right]^{2}\right) \sin ^{2}\left(\frac{\Delta m^{2} L}{4 E}\right)
$$

Oscillation probability $\nu_{\mu}$ to $\nu_{\tau}$ :

$$
P\left(\nu_{\mu} \rightarrow \nu_{\tau}\right)=\left(\frac{1}{4}\left[\sin \left(2 \theta_{C-\text { odd }}\right)+\sin \left(2 \theta_{C \text {-even }}\right)\right]^{2}\right) \sin ^{2}\left(\frac{\Delta m^{2} L}{4 E}\right)
$$

\section{Oscillation probability $\nu_{\mu}$ to $\bar{\nu}_{\tau}$ :}

$$
P\left(\nu_{\mu} \rightarrow \bar{\nu}_{\tau}\right)=\left(\frac{1}{4}\left[\sin \left(2 \theta_{C \text {-odd }}\right)-\sin \left(2 \theta_{C \text {-even }}\right)\right]^{2}\right) \sin ^{2}\left(\frac{\Delta m^{2} L}{4 E}\right)
$$

where $L$ is the distance travelled by the neutrinos and $E$ is their energy. Angles $\theta_{C \text {-odd }}$ and $\theta_{C \text {-even }}$ are the effective mixing angles for states that are odd and even, respectively, under charge conjugation operator $C$ in flavour space. They are related to $\Delta m^{2}, \sin ^{2} 2 \theta$, and the SME coefficients through the following relations:

$$
\tan 2 \theta_{C-o d d}=\frac{\Delta m^{2} \sin 2 \theta}{\left(\left(1.6\left(\tilde{g}_{\mu \bar{\mu}}^{Z T}-\tilde{g}_{\tau \bar{\tau}}^{Z T}\right)+2.7\left(\left(c_{L}\right)_{\mu \mu}^{T T}-\left(c_{L}\right)_{\tau \tau}^{T T}\right)\right) E^{2}+\Delta m^{2} \cos 2 \theta\right)}
$$

and

$$
\tan 2 \theta_{C \text {-even }}=\frac{\Delta m^{2} \sin 2 \theta}{\left(\left(-1.6\left(\tilde{g}_{\mu \bar{\mu}}^{Z T}-\tilde{g}_{\tau \bar{\tau}}^{Z T}\right)+2.7\left(\left(c_{L}\right)_{\mu \mu}^{T T}-\left(c_{L}\right)_{\tau \tau}^{T T}\right)\right) E^{2}+\Delta m^{2} \cos 2 \theta\right)}
$$

The aim of the analysis is to fit the data to the current model and thereby constrain the parameters $\tilde{g}_{\mu \bar{\mu}}^{Z T}, \tilde{g}_{\tau \bar{\tau}}^{Z T},\left(c_{L}\right)_{\mu \mu}^{T T}$, and $\left(c_{L}\right)_{\tau \tau}^{T T}$ that define the model.

The present analysis uses all the data obtained in a $10.56 \times 10^{20}$ PoT exposure obtained by MINOS in the FHC Low Energy running where the beam is composed of $91.7 \%$ muon 
neutrinos and $7 \%$ muon antineutrinos. Besides this there is also a small fraction of electron neutrinos and antineutrinos which are not considered for this analysis. We are able to place limits on the $\tilde{g}$-type and $c$-type parameters by fitting the neutrino and antineutrino spectra simultaneously using a binned log-likelihood. The $\tilde{g}$-type coefficients are responsible for the mixing between muon neutrinos and muon antineutrinos. These oscillations are energy dependent and would be seen as an excess of $\bar{\nu}_{\mu}$ events at the FD as compared to that predicted from the standard oscillations. The energy at which the excess is expected decreases when the value of these parameters is increased (Section 2.4). The $c$-type coefficients do not allow mixing between neutrinos and antineutrinos. They only change the mixing between neutrino states or between antineutrino states as compared to the standard two-flavour oscillation model. Therefore a deviation in the observed FD spectra as compared to the prediction from the SM could be due to non-zero c-type coeffcients. As is evident from equations 6.5 and 6.6 the parameters $\tilde{g}_{\mu \bar{\mu}}^{Z T}$ and $\tilde{g}_{\tau \bar{\tau}}^{Z T}$ are degenerate as also are the parameters $\left(c_{L}\right)_{\mu \mu}^{T T}$ and $\left(c_{L}\right)_{\tau \tau}^{T T}$. So we can perform a fit to the combination of parameters $\left|\tilde{g}_{\mu \bar{\mu}}^{Z T}-\tilde{g}_{\tau \bar{\tau}}^{Z T}\right|$, $\left(\left(c_{L}\right)_{\mu \mu}^{T T}-\left(c_{L}\right)_{\tau \tau}^{T T}\right)$, while marginalising over $\Delta m^{2}$ and $\sin ^{2} 2 \theta$. Limits on individual SME coefficients can also be obtained by setting all other SME coefficients to zero, and performing a fit to that coefficient, marginalised over $\Delta m^{2}$ and $\sin ^{2} 2 \theta$.

This chapter describes the selection procedure for neutrino and antineutrino events at the ND and the method to predict a FD spectrum. Then the details of the fitting framework and the systematic uncertainties that can affect the measurement of the individual SME parameters is given. For simplicity the framework is tested assuming that only $\tilde{g}_{\mu \bar{\mu}}^{Z T}$ coefficients are non-zero. The effect of the c-type parameters on the FD spectra is discussed later. Finally the results obtained by fit to the data are presented in two ways:

1) The fit is performed to the individual SME parameters, marginalised over $\Delta m^{2}$ and $\sin ^{2} 2 \theta$, assuming all other parameters to be zero. This will provide limits to the individual SME parameters.

2) The fit is performed to $\left|\tilde{g}_{\mu \bar{\mu}}^{Z T}-\tilde{g}_{\tau \bar{\tau}}^{Z T}\right|$ and $\left(\left(c_{L}\right)_{\mu \mu}^{T T}-\left(c_{L}\right)_{\tau \tau}^{T T}\right)$, marginalised over $\Delta m^{2}$ and 
$\sin ^{2} 2 \theta$ to show the parameter space constrained by the model.

\subsection{Event Selection}

We used $\nu_{\mu}$ as well as $\bar{\nu}_{\mu}$ for this analysis. The event selection was kept unchanged from that used for the previous MINOS charged-current analyses [97, 143, 144, and is discussed below.

\subsubsection{The Antineutrino Dataset}

It has been previously discussed in Section 5.1 that we use charged-current interactions to identify antineutrino events. Positively charged muon is formed in the following interaction

$$
\bar{\nu}_{\mu}+N \rightarrow \mu^{+}+\text {hadrons }
$$

and is defocused in the detector because of the magnetic field. More details can be found in Section 5.1. The energy spectrum for the ND in this case is obtained using all muon antineutrino data obtained in $10.56 \times 10^{20}$ PoT exposure. The size of systematic uncertainty is reduced as compared to the previous analysis because of the reduction in size of one of the largest systematics, the decay pipe systematic. This will be discussed later in Section 6.6.

\subsubsection{The Neutrino Dataset}

We again use charged-current interactions to identify neutrino events. The lepton formed in this case is a negatively charged muon,Eq. 6.8, which is focused by the detector's magnetic field.

$$
\nu_{\mu}+N \rightarrow \mu^{-}+\text {hadrons }
$$

The presence of a track is the basic requirement for identifying neutrino events. The track is long for neutrinos with high energy, and may be short for events with low neutrino energy. But not all events with a reconstructed track are due to charged-current interactions. For 
example, sometimes a hadronic shower in a neutral-current interaction may be reconstructed as a track. The PID algorithm in MINOS is developed to identify muon tracks and reduce the background as much as possible.

The basic preselection applied is the same as that described in Section 5.1.1. The four input variables that separate neutral-current and charged-current events are shown in Fig. 6.1. They are:

- Number of scintillator planes - The tracks formed by muons in the detector are long and smooth as compared to hadronic particles, which interact more strongly in the detector and form a shorter track.

- Mean energy deposited per strip - The charge deposited by muons in the detector is almost constant (one MIP) in each plane. On the other hand hadronic showers give a higher mean energy deposition. The first $30 \%$ of planes after the track vertex are excluded when calculating the mean.

- Signal fluctuation parameter - Tracks formed due to hadronic activity have larger fluctuations in energy deposition as compared to muons. All hits within four scintillator strips of the reconstructed track, after the first $30 \%$ of planes, are sorted by pulse height. The variable is defined as the mean of the lowest pulse-height strips divided by the mean of the highest pulse-height strips. A larger value of the parameter indicates more uniform energy deposition.

- Transverse profile parameter - Energy deposited by muon tracks is typically confined to one strip per plane, while hadronic showers are wider. The transverse profile parameter is defined as the fraction of energy deposited within a 4 strip window assigned to the reconstructed track. This is calculated by excluding $50 \%$ of track planes closest to the vertex.

The above four variables are combined using a $k$-Nearest-Neighbours $(k N N)$ technique. Monte Carlo is used to form a training sample of events, and for each event to be clas- 

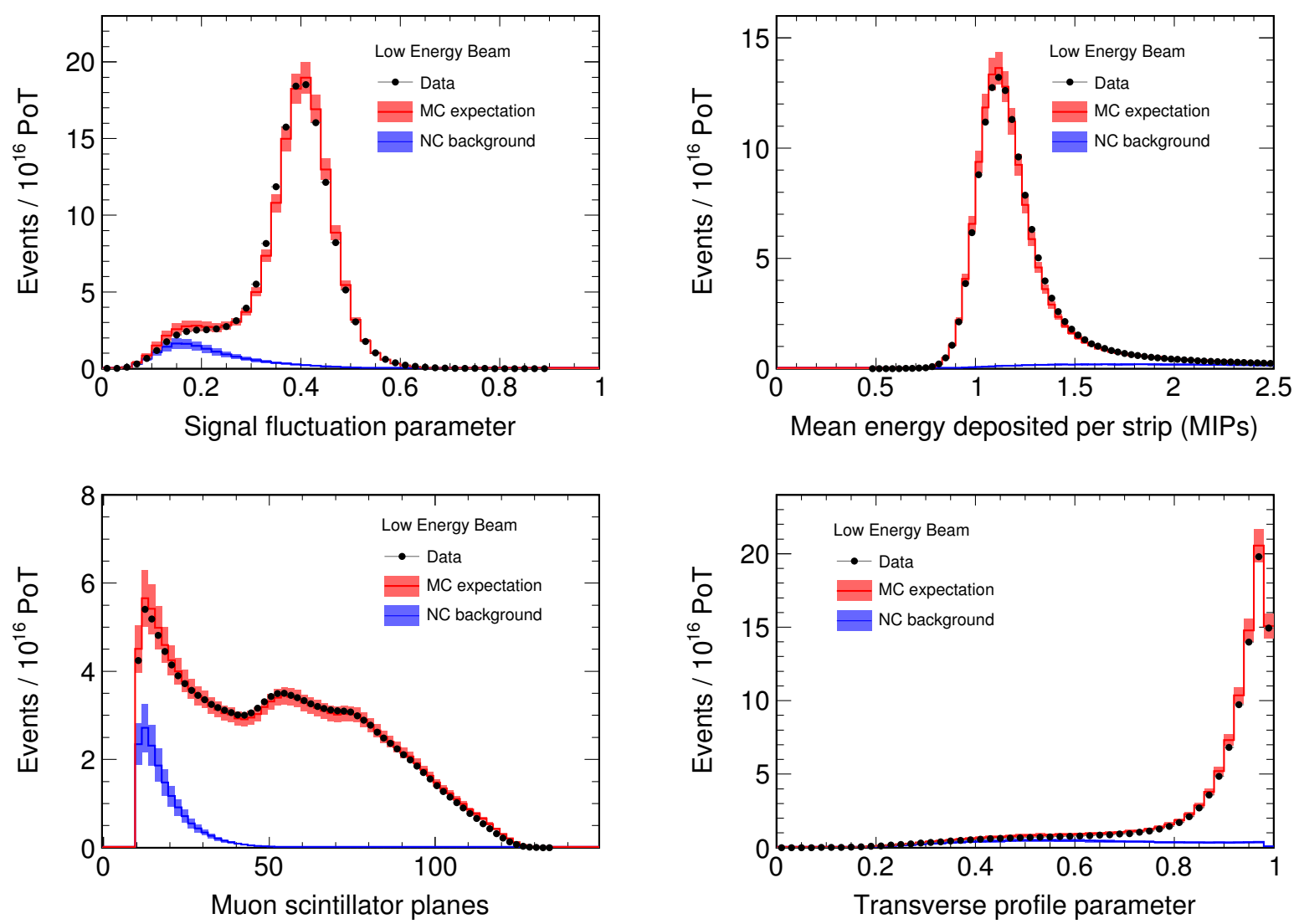

Figure 6.1: Distributions of the four variables used as input to the primary CC selection. Monte Carlo prediction is shown in red, with the shaded portion showing the systematic error associated with the beam flux. The expected neutral-current distribution is shown in blue. The black points show the distributions in the ND data. The data and Monte Carlo show good agreement.

sified the $k$ ( $k$ is 80 in this case) nearest training events in the parameter space are found. The output of the PID algorithm is then simply the fraction of these events that were due to $\nu_{\mu}$-CC interaction. The distribution of the $k \mathrm{NN}$ output $(\mathrm{R})$ is shown in Fig. 6.2 .

An additional PID was developed to improve selection at low energies (0-5 GeV). The detailed description can be found in [145, 146]. The input variables are:

- Number of muon scintillator planes - Same as defined earlier for the variable R.

- End Pulse Height - The energy deposition at the end of the track may be quite large for a track from a hadronic interaction compared to a muon track. This variable is defined as the total energy deposit in the last five planes of the track. 


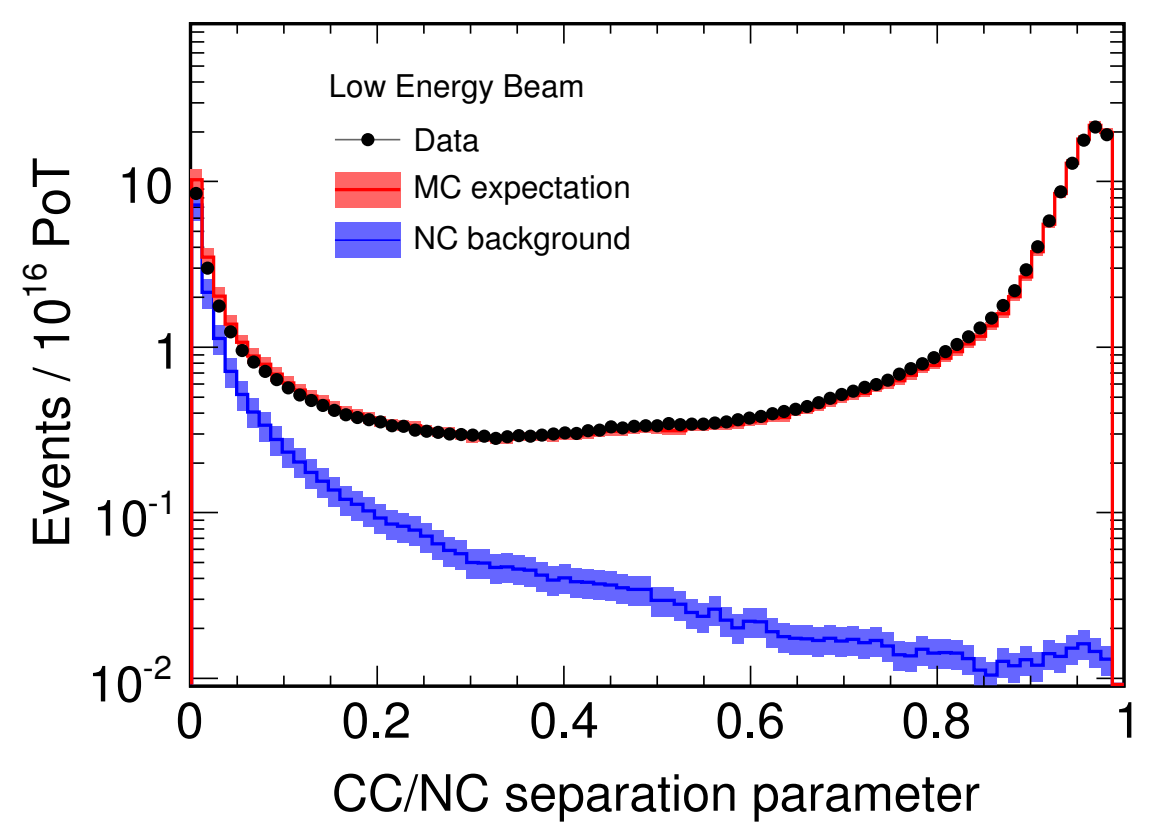

Figure 6.2: Output of $k \mathrm{NN}$ formed from variables in Fig. 6.1. The data and Monte Carlo show good agreement.

- Degree of scattering - Hadronic tracks scatter much more and are less straight as compared to muon tracks. This variable is calculated by combining the Pearson (correlation) coefficients of the track in the $\mathrm{U}$ and $\mathrm{V}$ views.

The three variables are shown in Fig. 6.3. They are again combined using the $k \mathrm{NN}$ technique. The distribution of the $k \mathrm{NN}$ output $(\mathrm{J})$ in this case is shown in Fig. 6.4. The final PID is constructed so as to select an event that passes either of the two selectors. The cut to be applied to this PID is optimised to maximise the product of efficiency and purity, $\epsilon \times p$. The cut position that miximises this product is identified to be:

$$
(R>0.3) \text { OR }(J>0.5) .
$$

The selection efficiency and level of contamination achieved by this selector as a function of reconstructed energy in the FD Monte Carlo is shown in Fig. 6.5. The selector performs excellent $\mathrm{CC} / \mathrm{NC}$ separation for energies above $1 \mathrm{GeV}$. 

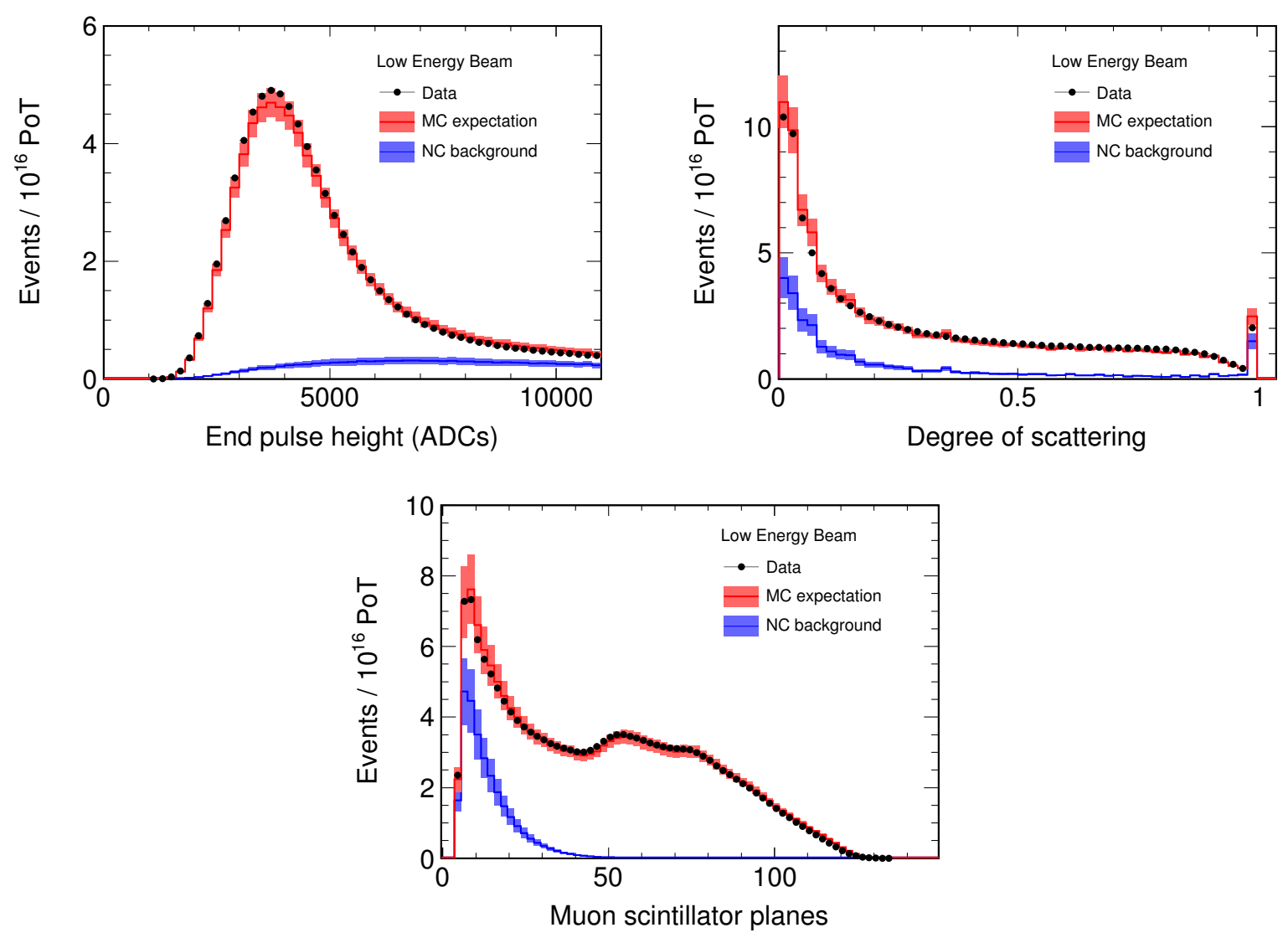

Figure 6.3: Distributions of the input variables to the auxiliary CC selection. Conventions are as given in Fig. 6.1.

\subsection{Far Detector Prediction}

The beam matrix extrapolation method, described earlier in Sec. 5.3, is used to predict the unoscillated neutrino and antineutrino flux in true energy at the FD (Fig. 5.13). Both $\nu_{\mu}$ and $\bar{\nu}_{\mu}$ events are extrapolated independently. After obtaining the total flux at the FD the next step is to apply appropriate weights to the antineutrino and neutrino fluxes to obtain the FD predicted spectra. To obtain FD $\bar{\nu}_{\mu}$ prediction, first, the cross-section and fiducial mass corrections are applied to the $\mathrm{FD} \nu_{\mu}$ and $\bar{\nu}_{\mu}$ fluxes. The $\nu_{\mu}$ flux is then weighted with the oscillation probability $\mathrm{P}\left(\nu_{\mu} \rightarrow \bar{\nu}_{\mu}\right)$, and the $\bar{\nu}_{\mu}$ flux is weighted with the survival probability $\mathrm{P}\left(\bar{\nu}_{\mu} \rightarrow \bar{\nu}_{\mu}\right)$. The spectra are then multiplied with the $\mathrm{FD} \bar{\nu}_{\mu}$ efficiency, and the spectrum in true energy is converted to reconstructed energy by multiplying with the 


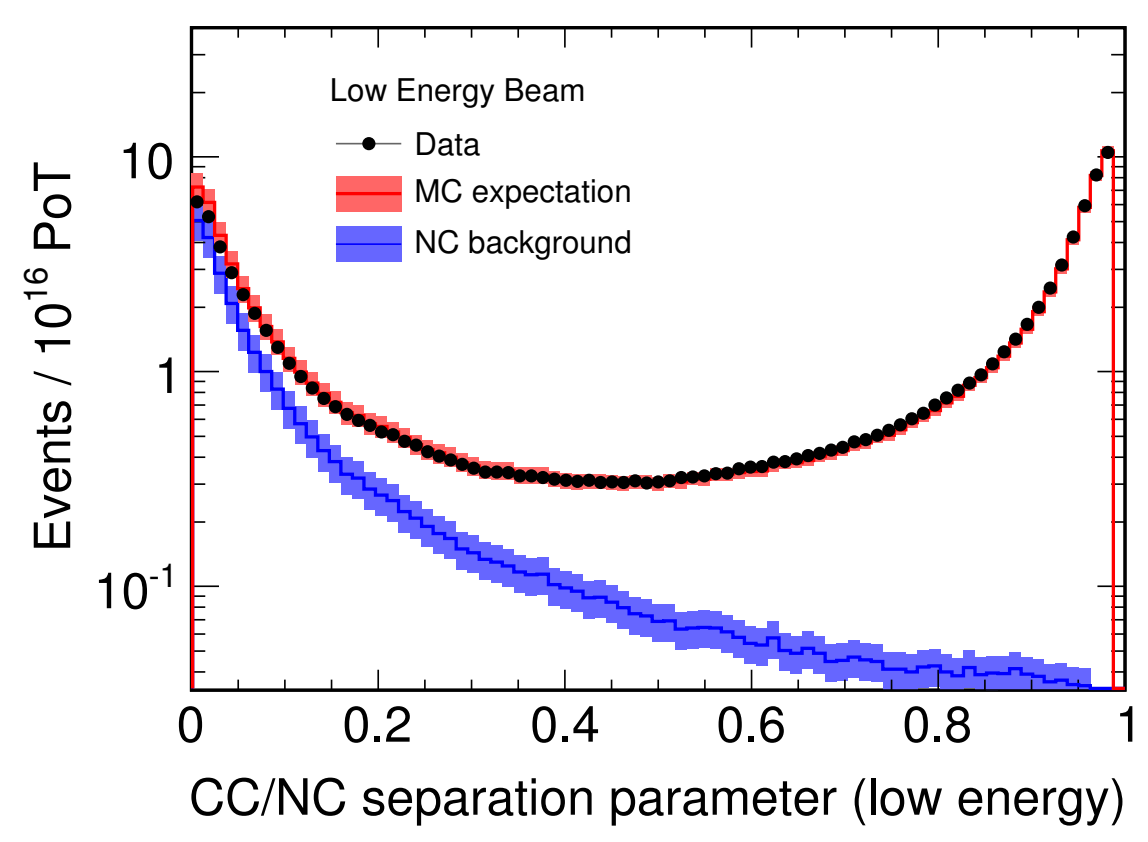

Figure 6.4: Output of the $\mathrm{kNN}$ formed from variables in Fig. 6.3. The data and Monte Carlo show good agreement.

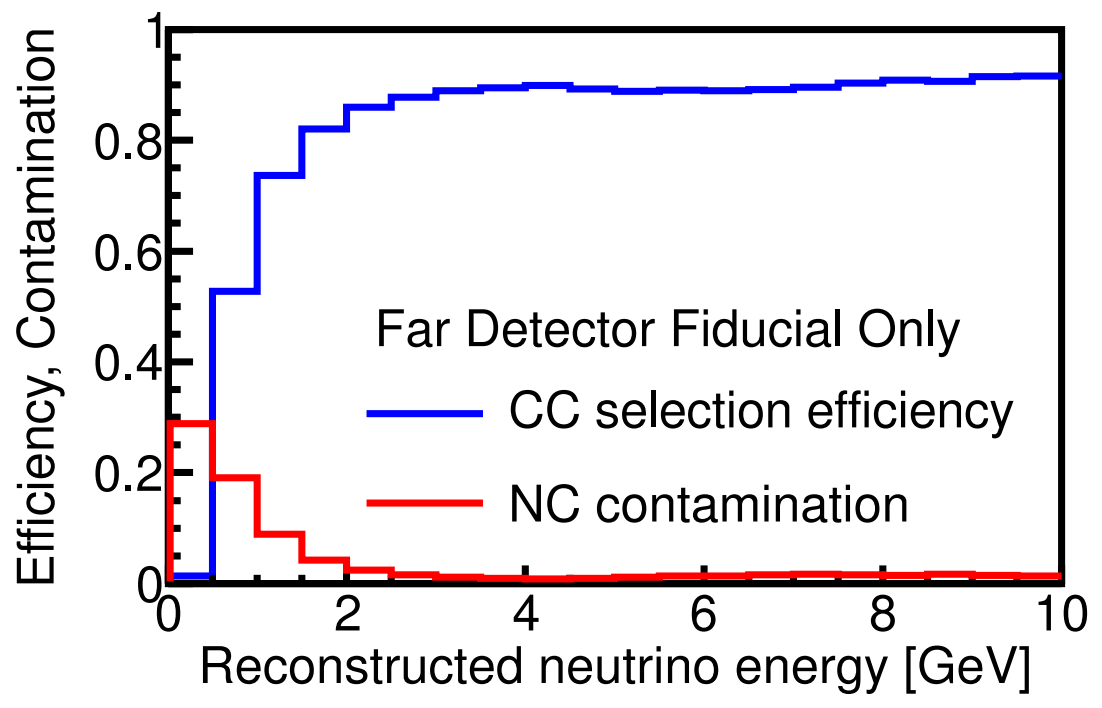

Figure 6.5: Efficiency (blue) and contamination (red) achieved by the charged-current selection at the far detector as a function of reconstructed energy. Above $1 \mathrm{GeV}$ very high efficiency and very low contamination is obtained. 
2-dimensional matrix which transforms between true and reconstructed energy. This gives the number of $\bar{\nu}_{\mu}$ events transitioning from $\nu_{\mu}$ events, and the number of $\bar{\nu}_{\mu}$ events surviving at the FD, respectively. There is also a small probability of $\nu_{\mu}$ oscillating into $\nu_{\tau}$ and $\bar{\nu}_{\tau}$, and $\bar{\nu}_{\mu}$ oscillating to $\nu_{\tau}$ and $\bar{\nu}_{\tau}$ (Eq. 2.28 and 2.29p. The backgrounds for the antineutrino sample are $\bar{\nu}_{\tau}$ events, $\nu_{\mu}$ misidentified as $\bar{\nu}_{\mu}$ (also called 'wrong-sign' or WS background), and NC events. The expected background is also added to the predicted FD spectrum to obtain the total $\bar{\nu}_{\mu}$ spectrum expected at the FD. (Fig. 6.6). A similar procedure is used to obtain the FD $\nu_{\mu}$ prediction (Fig. 6.7). The backgrounds for the neutrino sample are $\nu_{\tau}$ events, $\bar{\nu}_{\mu}$ misidentified as $\nu_{\mu}$, and NC events.

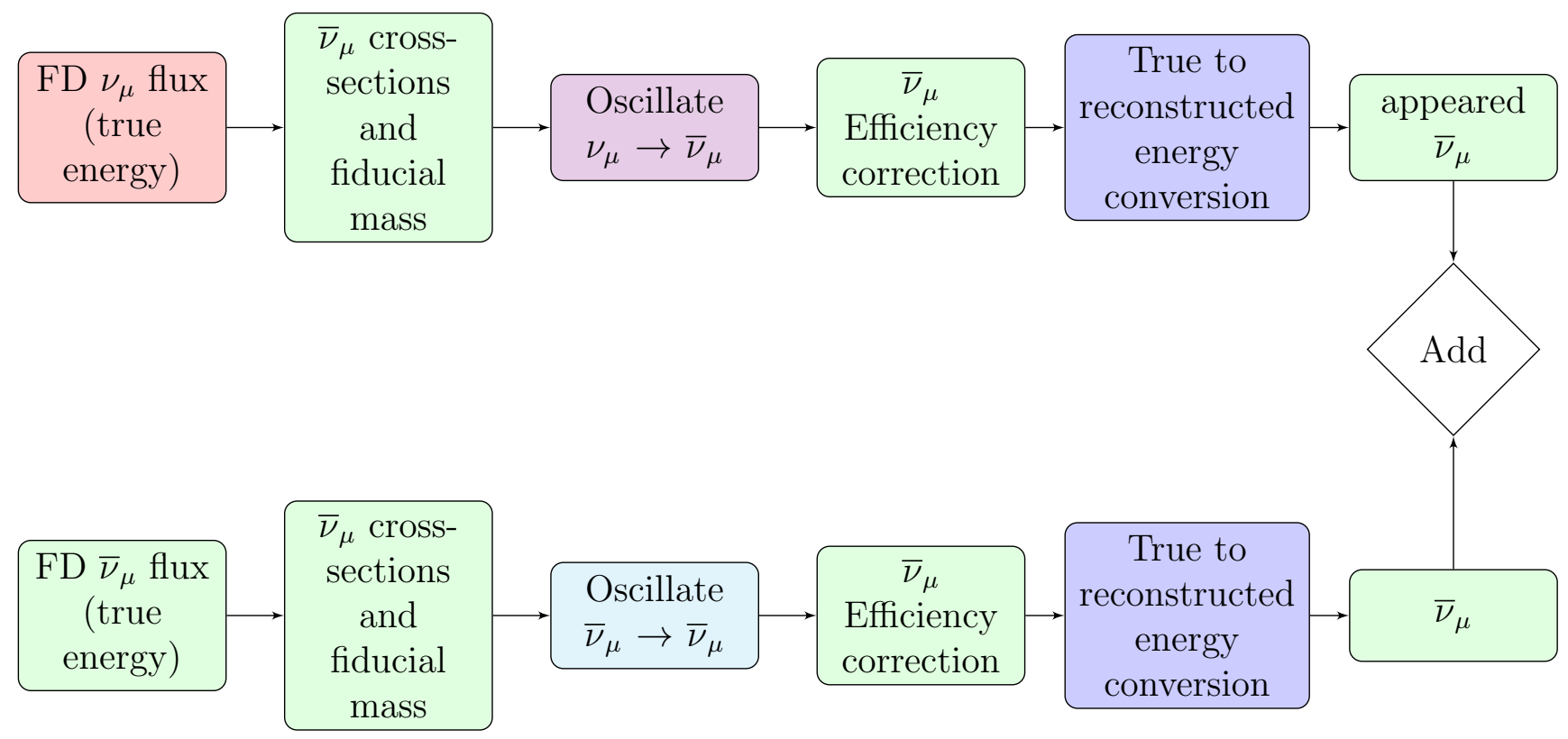

Figure 6.6: Steps to obtain the FD $\bar{\nu}_{\mu}$ prediction in reconstructed energy. The backgrounds: $\bar{\nu}_{\tau}$, 'wrong-sign' $\nu_{\mu}$ 's and NC backgrounds, are also added after applying oscillation weights appropriately.

Fig. 6.8 shows the $\mathrm{FD} \bar{\nu}_{\mu}$ prediction with different values of $\tilde{g}_{\mu \bar{\mu}}^{Z T}$, assuming all other parameters to be zero. A feature of this model is that there is a threshold in energy above which the Lorentz and $\mathcal{C P} \mathcal{T}$ violating parameters dominate and the dominant oscillations change from $\nu_{\mu} \rightarrow \nu_{\tau}$ to $\nu_{\mu} \rightarrow \bar{\nu}_{\mu}$. The energy threshold varies as $\sqrt{\Delta m^{2} / 1.6 \mid \tilde{g}_{\alpha \bar{\alpha}}^{Z T}} \mathrm{GeV}$. Therefore as $\left|\tilde{g}_{\alpha \bar{\alpha}}^{Z T}\right|$ increases the threshold for $\nu_{\mu} \rightarrow \bar{\nu}_{\mu}$ oscillations decreases and we see a 


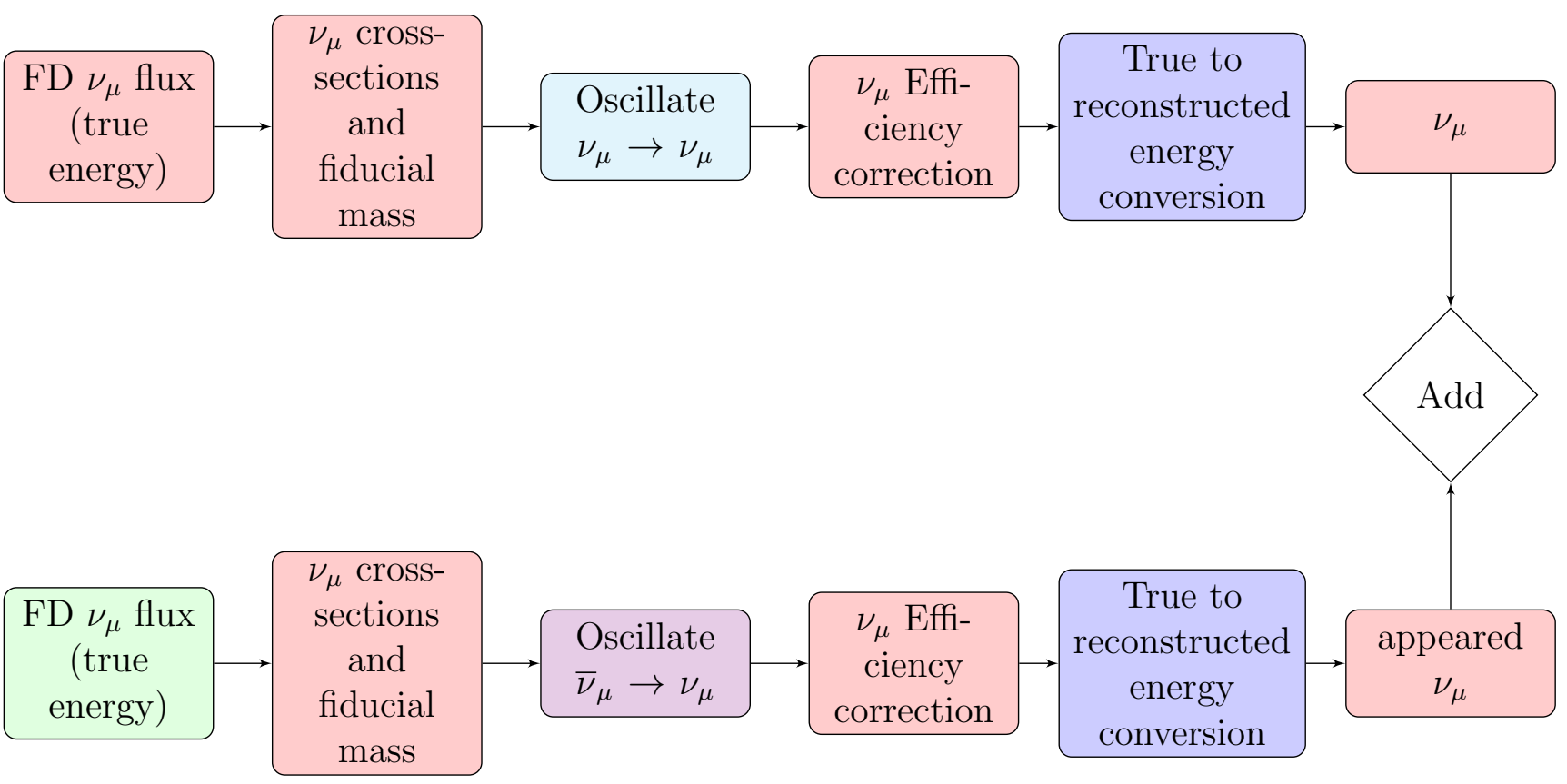

Figure 6.7: Steps to obtain the FD $\nu_{\mu}$ prediction in reconstructed energy. The backgrounds: $\nu_{\tau}$, 'wrong-sign' $\bar{\nu}_{\mu}$ 's and NC backgrounds, are also added after applying oscillation weights appropriately.

peak above the standard oscillations spectrum due to $\bar{\nu}_{\mu}$ appearance (from $\nu_{\mu}$ oscillating to $\bar{\nu}_{\mu}$ ). On the other hand, the $\nu_{\mu}$ prediction, shown by Fig. 6.9, does not show such a peak since the number of $\bar{\nu}_{\mu}$ events is much less as compared to $\nu_{\mu}$ events. Therefore the small number of $\bar{\nu}_{\mu}$ events oscillating to $\nu_{\mu}$ events does not change the $\nu_{\mu}$ spectrum much.

\subsection{Generating Simulated Data}

Simulated data were used to test whether the extrapolation framework is working correctly, to calculate the expected sensitivity to the Lorentz and $\mathcal{C P} \mathcal{T}$ violating parameters, and to evaluate the effect of systematic uncertainties on the measurements. So that it serves as a worthwhile cross-check, the simulated FD data is produced in a way significantly different from the FD prediction made using the ND data, described in Section6.2. It is produced by reweighting the unoscillated spectrum in FD Monte Carlo by the oscillation probability. True fiducial neutrino and antineutrino fluxes are generated using Monte Carlo. By dividing the neutrino flux by the antineutrino flux, a set of weights as a function of true energy is 


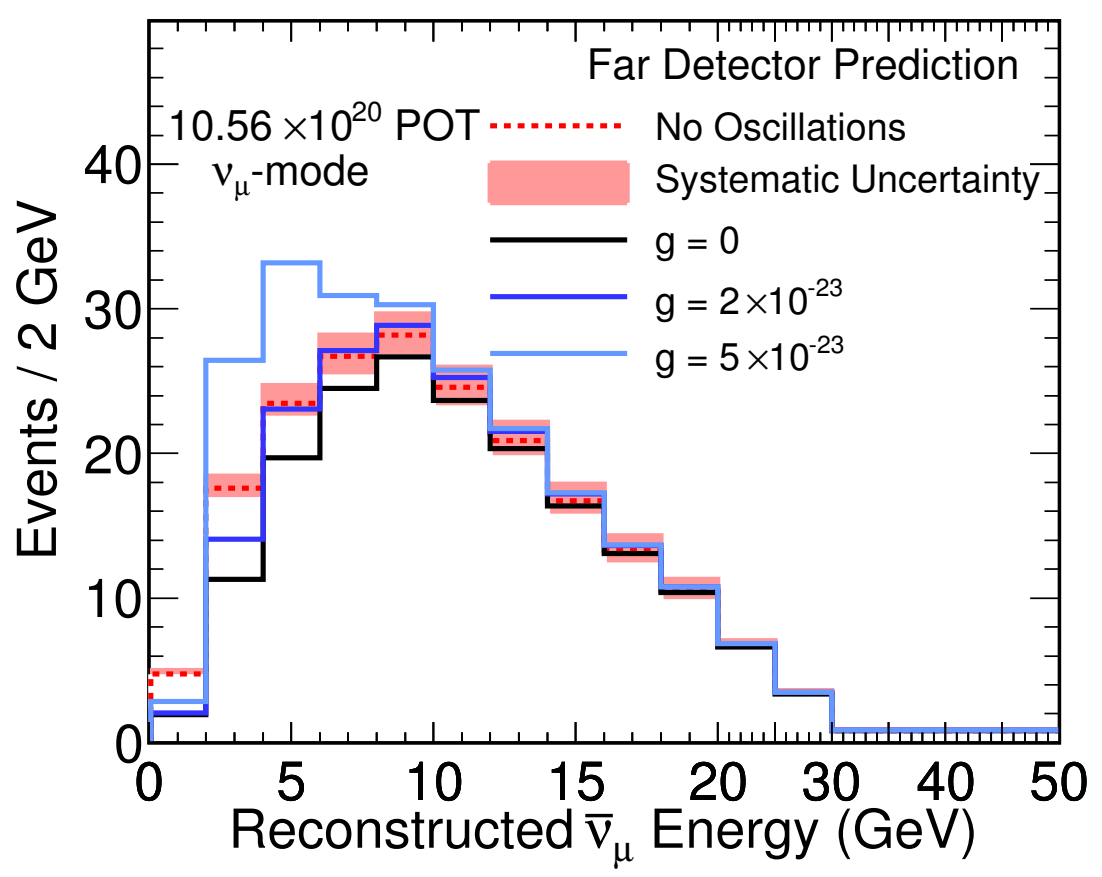

Figure 6.8: $\mathrm{FD} \bar{\nu}_{\mu}$ prediction with $\Delta m^{2}=2.32 \times 10^{-3} \mathrm{eV}^{2}, \sin ^{2} 2 \theta=0.97$, and different values of $\tilde{g}_{\alpha \bar{\alpha}}^{Z T}$, where $\alpha$ can be $\mu$ or $\tau$. As the value of $\mathrm{g} \equiv \tilde{g}_{\alpha \bar{\alpha}}^{Z T}$ increases, a peak is seen in the FD predicted spectrum due to lowering of the threshold for transitions.

produced that will transform between them. Then, selected events are looped through. If the event is an antineutrino, it is first added to the far detector spectrum histograms weighted by its survival probability, and then the event is added again, this time weighted by the neutrino-antineutrino flux ratio and the $\nu_{\mu}$ to $\bar{\nu}_{\mu}$ transition probability,

$$
w_{\bar{\nu}}=\frac{f_{\nu}(E)}{f_{\bar{\nu}}(E)} P\left(\nu_{\mu} \rightarrow \bar{\nu}_{\mu}\right)
$$

where $w_{\bar{\nu}}$ is the weight applied to the antineutrino to make the transitioned antineutrino, $f_{\nu / \bar{\nu}}$ are the fluxes, and $E$ is the energy.

If the event is a neutrino, it is first added to the far detector spectrum histograms weighted by the $\nu_{\mu}$ survival probability, and then the event is added again, this time weighted by the 


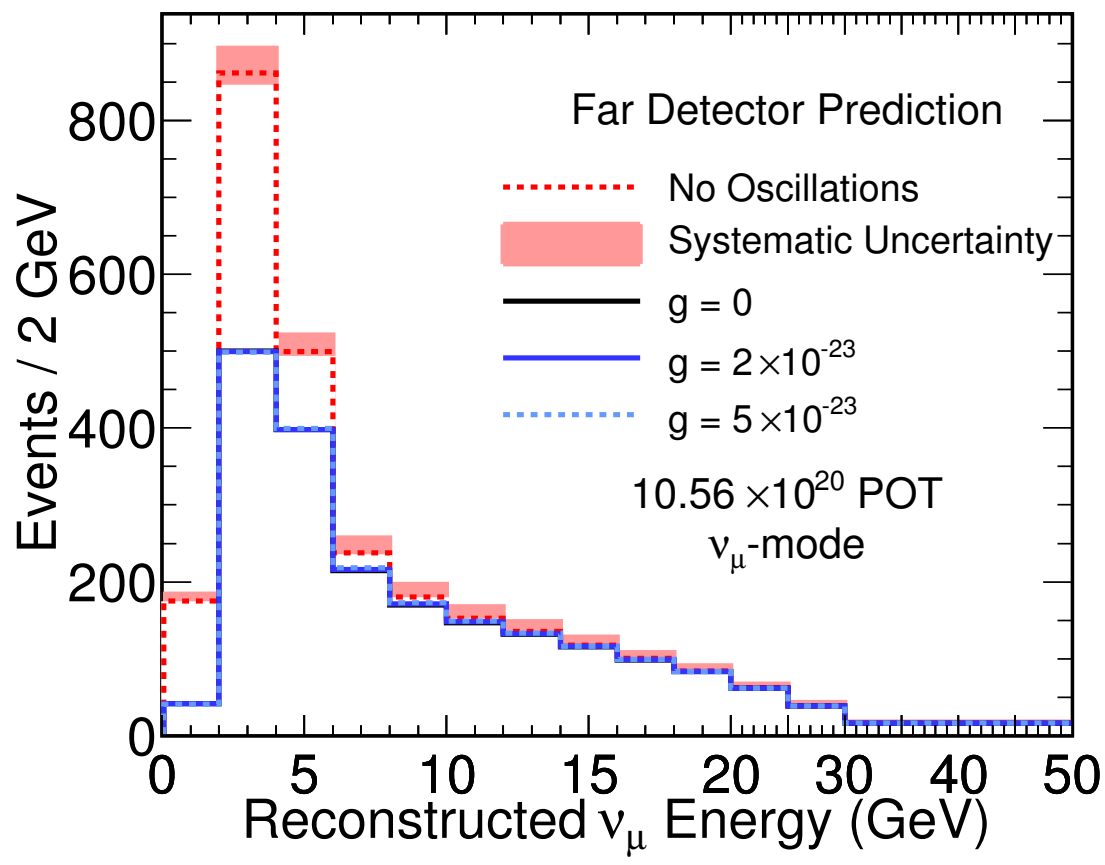

Figure 6.9: FD $\nu_{\mu}$ prediction with $\Delta m^{2}=2.32 \times 10^{-3} \mathrm{eV}^{2}, \sin ^{2} 2 \theta=0.97$, and different values of $\mathrm{g} \equiv \tilde{g}_{\alpha \bar{\alpha}}^{Z T}$.

antineutrino-neutrino flux ratio and the $\bar{\nu}_{\mu}$ to $\nu_{\mu}$ transition probability,

$$
w_{\nu}=\frac{f_{\bar{\nu}}(E)}{f_{\nu}(E)} P\left(\bar{\nu}_{\mu} \rightarrow \nu_{\mu}\right)
$$

where $w_{\nu}$ is the weight applied to the neutrino to make the transitioned neutrino. This gives us the simulated $\bar{\nu}_{\mu}$ and $\nu_{\mu}$ FD histograms.

\subsection{Cross-check of the extrapolation framework}

To verify that the extrapolation is performed correctly the simulated FD data and the FD prediction are compared. The two are expected to be the same for the same values of oscillation parameters. Four sets of simulated data and predictions were generated with $\Delta \mathrm{m}^{2}$ $=2.32 \times 10^{-3} \mathrm{eV}^{2}, \sin ^{2} 2 \theta=0.97$, and different values of $\tilde{g}_{\mu \bar{\mu}}^{Z T}: \tilde{g}_{\mu \bar{\mu}}^{Z T}=0,2 \times 10^{-24}, 2 \times 10^{-23}$, and $2 \times 10^{-22}$. The other SME parameters were set to zero for simplicification. The values 
of $\tilde{g}_{\mu \bar{\mu}}^{Z T}$ chosen are arbitrary and are intended to check that the framework works well at all values of SME parameters, ranging from very small to very large. The simulated data and prediction are found to be in good agreement, shown in Fig. 6.10.
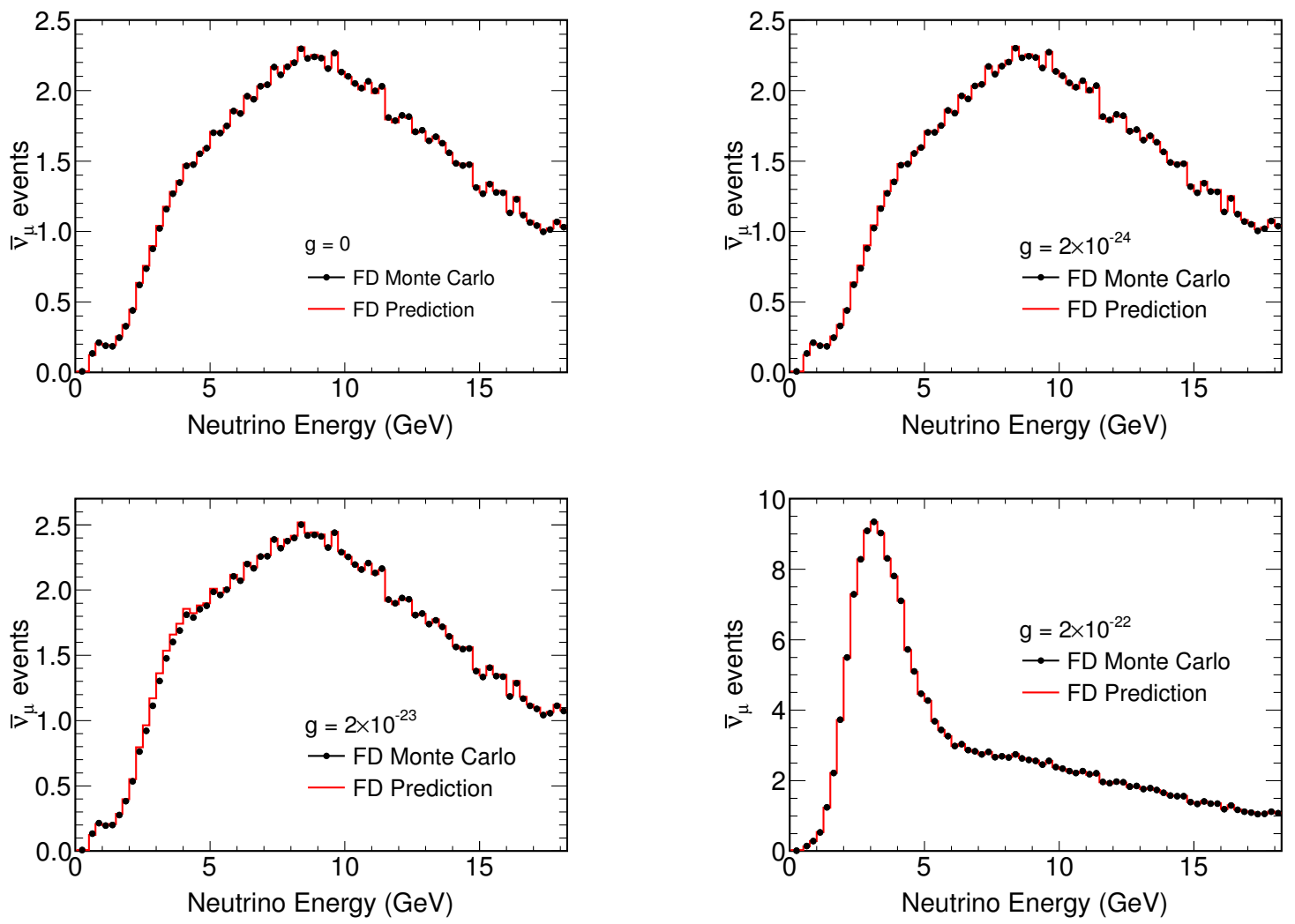

Figure 6.10: $\bar{\nu}_{\mu}$ spectra showing agreement between FD prediction and simulated data. The data and prediction are produced with $\Delta m^{2}=2.32 \times 10^{-3} \mathrm{eV}^{2}, \sin ^{2} 2 \theta=0.97$, and different values of $\mathrm{g} \equiv \tilde{g}_{\mu \bar{\mu}}^{Z T}$.

\subsection{Fitting Procedure}

A fit to the simulated FD data was done for each case by performing a log-likelihood fit to the data. The function defined below is minimised to get the best-fit parameters:

$$
-2 \log \lambda=2 \sum_{i=1}^{n}\left[P_{i}-D_{i}+D_{i} \ln \left(\frac{D_{i}}{P_{i}}\right)\right]
$$


where $P_{i}$ is the expected number of events based on the FD prediction and $D_{i}$ is the number of events from FD data in bin $i$. Minuit is used to minimise the above function. The values of the parameters $\Delta m^{2}, \sin ^{2} 2 \theta$, and $\left|\tilde{g}_{\mu \bar{\mu}}^{Z T}\right|$ returned by Minuit for each case, and summarised in Table. 6.1, are very close to the input values. The input $\Delta m^{2}$ and $\sin ^{2} 2 \theta$ in each case is $2.32 \times 10^{-3} \mathrm{eV}^{2}$ and 0.97 , respectively. Only the magnitude of the parameter $\tilde{g}_{\mu \bar{\mu}}^{Z T}$ is shown here because we are not sensitive to its sign.

\begin{tabular}{l|c|c|c} 
Input & \multicolumn{3}{|c}{ Best-fits } \\
\hline$\tilde{g}_{\mu \bar{\mu}}^{Z T}$ & $\Delta m^{2} \times 10^{-3} \mathrm{eV}^{2}$ & $\sin ^{2} 2 \theta$ & $\left|\tilde{g}_{\mu \bar{\mu}}^{Z T}\right|$ \\
\hline 0 & $2.31 \pm 0.04$ & $0.975 \pm 0.03$ & $7.6 \times 10^{-34} \pm 3.5 \times 10^{-23}$ \\
$2 \times 10^{-24}$ & $2.31 \pm 0.04$ & $0.975 \pm 0.03$ & $(2.2 \pm 22.1) \times 10^{-24}$ \\
$2 \times 10^{-23}$ & $2.31 \pm 0.05$ & $0.975 \pm 0.03$ & $(1.9 \pm 0.9) \times 10^{-23}$ \\
$2 \times 10^{-22}$ & $2.29 \pm 0.01$ & $0.986 \pm 0.06$ & $(1.7 \pm 0.2) \times 10^{-22}$
\end{tabular}

Table 6.1: Best-fit values of the parameters $\Delta m^{2}, \sin ^{2} 2 \theta$, and $\left|\tilde{g}_{\mu \bar{\mu}}^{Z T}\right|$ obtained from the fit to simulated FD data generated at four different values of $\tilde{g}_{\mu \bar{\mu}}^{Z T}$.

In order to find our sensitivity to the parameter $\tilde{g}_{\mu \bar{\mu}}^{Z T}$, a one-dimensional likelihood distribution is obtained by varying the parameter $\tilde{g}_{\mu \bar{\mu}}^{Z T}$ and calculating the value of the loglikelihood in Eq. 6.12, The parameters $\Delta m^{2}$ and $\sin ^{2} 2 \theta$ are allowed to vary in the fit as free parameters, using the procedure known as marginalisation. We can construct confidence levels from the likelihood curve by using the difference of $-2 \log \lambda$ at each point compared to the best fit point ( called $-2 \Delta \log \lambda$ ). The values of $-2 \Delta \log \lambda=1,4$, and 9 correspond to $1 \sigma$, $2 \sigma$, and $3 \sigma$ confidence levels respectively [42]. The top left $-2 \Delta \log \lambda$ distribution in Fig. 6.11 is drawn using the simulated data generated with $\tilde{g}_{\mu \bar{\mu}}^{Z T}=0$. Therefore, assuming that there is no Lorentz and $\mathcal{C P} \mathcal{T}$ violation, we can constrain the $\tilde{g}_{\mu \bar{\mu}}^{Z T}$ coefficients to $<3.1 \times 10^{-23}$ at 3 standard deviations $(3 \sigma)$. The top right distribution, drawn with $\tilde{g}_{\mu \bar{\mu}}^{Z T}=2 \times 10^{-24}$, also shows a very similar sensitivity because the antineutrino spectrum at this value of $\tilde{g}_{\mu \bar{\mu}}^{Z T}$ is almost the same as that with $\tilde{g}_{\mu \bar{\mu}}^{Z T}=0$. For $\tilde{g}_{\mu \bar{\mu}}^{Z T}=2 \times 10^{-23}$ (bottom left) and $2 \times 10^{-22}$ (bottom right) we can see the curves shifting towards right, where the minimum in the curve corresponds to the best-fit value. We observe that for smaller values of the parameter $\tilde{g}_{\mu \bar{\mu}}^{Z T}$ the best-fit point is very close to the input value, whereas for very large value of $\tilde{g}_{\mu \bar{\mu}}^{Z T}\left(=2 \times 10^{-22}\right)$ the 

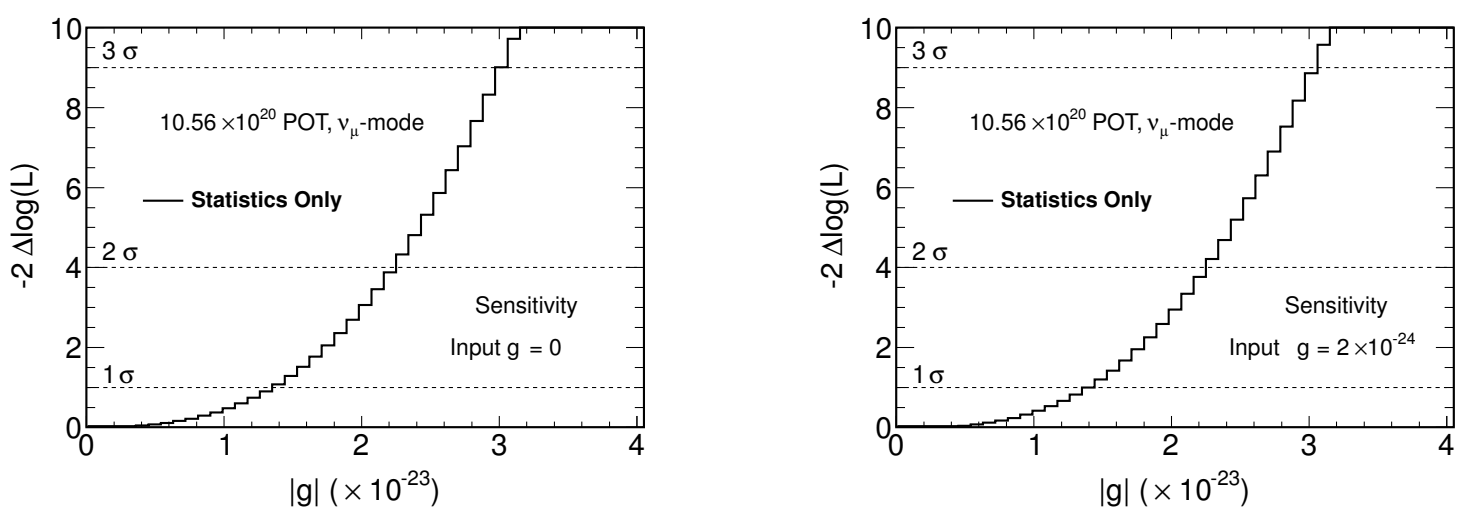

$\lg \mid\left(\times 10^{-23}\right)$
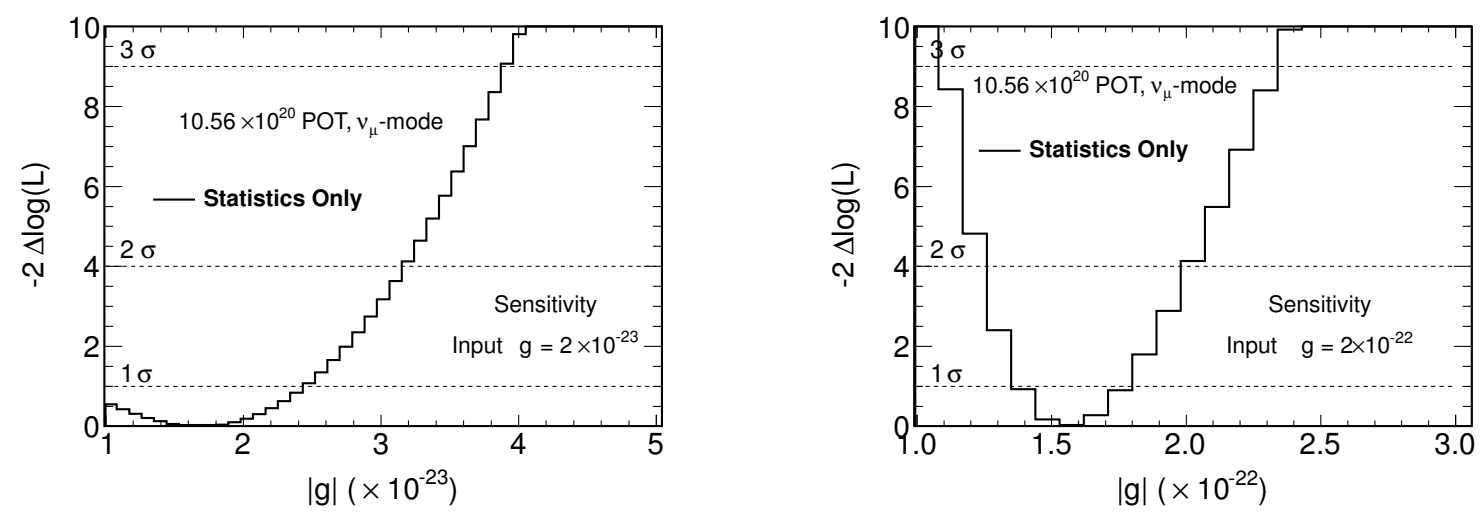

Figure 6.11: One-dimensional $-2 \Delta \log \lambda$ distribution for the parameter $\tilde{g}_{\mu \bar{\mu}}^{Z T}$ obtained by fitting simulated data generated at $\tilde{g}_{\mu \bar{\mu}}^{Z T}=0,2 \times 10^{-24}, 2 \times 10^{-23}$, and $2 \times 10^{-22}$. The dashed lines show the 1- $\sigma, 2-\sigma$, and3- $\sigma$ limit. The minimum value of $-2 \Delta \log \lambda$ corresponds to the value of $\tilde{g}_{\mu \bar{\mu}}^{Z T}$ at which the simulated data was generated.

best-fit obtained is somewhat away from the input value. This is because for large $\tilde{g}_{\mu \bar{\mu}}^{Z T}$ the effective mixing angle becomes maximal (see Section 2.4, Fig. 2.2, and the fitter tends to give a near maximal value of $\sin ^{2} 2 \theta(=0.986$ in Table 6.1 $)$ at the cost of reducing the value of $\tilde{g}_{\mu \bar{\mu}}^{Z T}$. These curves only show the expected statistical uncertainty on the parameter $\tilde{g}_{\mu \bar{\mu}}^{Z T}$. In the next two sections the known systematic uncertainties are discussed and their effect on the results is presented.

\subsection{Systematic Uncertainties}

Since this analysis uses both neutrinos and antineutrinos the systematic uncertainties affecting the neutrino as well as the antineutrino sample were studied. The set of systematic 
uncertainties affecting antineutrinos is the same as described in Section 5.5 with the exception of the decay-pipe systematic. This is the uncertainty in the production of neutrinos from the decay of pions produced in the downstream part of the beamline, the major part of which comes from the interactions with the decay pipe. This systematic uncertainty was re-evaluated for this analysis. The uncertainties affecting the neutrinos and that due to downstream events are described below:

\subsubsection{Track Energy Scale}

The uncertainty on the measurement of muon momentum from range is $2 \%$ and that from curvature is $1 \%$. It is taken to be fully correlated between the two detectors.

\subsubsection{Shower Energy Scale}

The total relative uncertainty in the ND for $\nu_{\mu}$ sample is $1.85 \%$. The relative uncertainty in the FD is $1.05 \%$. The absolute shower energy uncertainty is energy dependent and is parametrised as $\left(6.6+3.5 \mathrm{e}^{-E_{s h w} / 1.44 G e V}\right) \%$. The various components in shower energy estimation have been discussed earlier in Section 5.5

\subsubsection{Backgrounds}

The uncertainty in the NC background is primarily due to mis-modelling of hadronic showers and neutral-current cross sections in the Monte Carlo. The wrong-sign background for the neutrino sample is the $\bar{\nu}_{\mu}$ events mis-identified as $\nu_{\mu}$ events. The method to quantify these systematics consists of comparing data and Monte Carlo energy spectra of all preselected events below the PID cut value. In this background-dominated region all data-MC discrepancy are attributed to the background events. The MC CC and NC samples are scaled independently to agree with data and the total scale is reported as an estimate of the uncertainty. The uncertainty on NC background is $11 \%$ and that on WS background is $1.5 \%$. 


\subsubsection{Normalisation}

The sources of the normalisation uncertainty have been discussed earlier in Section 5.5. For the neutrino sample this uncertainty was determined by performing an extensive manual scan of real and simulated neutrino CC events in both the detectors. This scan showed no evidence of an unmodelled difference between the detectors. The statistical precision of this scan is $1.3 \%$ and is taken as the systematic uncertainty in the relative efficiencies between the Near and Far detectors.

\subsubsection{Cross-sections}

Several cross sections uncertainties were evaluated as described in [147]. However, these uncertainties are very small and are also fully correlated between the two detectors. Therefore they have a very small effect on the final result.

\subsubsection{Flux Modelling}

This systematic is also evaluated as described in [148]. Since most of the flux uncertainties affect both detectors in the same way it does not impact the analysis.

\subsubsection{Downstream Events}

Uncertainty in production of muon antineutrinos from pions produced in the decay pipe is a non-trivial uncertainty for this analysis since it is very sensitive to small changes to the expected number of muon antineutrinos. In the previous FHC antineutrino analysis the decay pipe uncertainty was obtained by attributing the entire Data/MC discrepancy in the ND to the decay pipe component of the beam. A maximum uncertainty of $\pm 100 \%$ was obtained by this method, which is an overestimate. New constraints on this uncertainty were obtained by comparing the decay pipe component of the flux obtained with different physical models from MINOS and MINER $\nu \mathrm{A}$ MC samples [149]. MINOS experiment uses the FLUGG [122, 123, 124] MC generator for beam simulation. The fraction of the decay 
pipe component using FLUGG was compared with different models, shown in Fig. 6.12, Fig. 6.13 shows the ratio of this component to FLUGG.

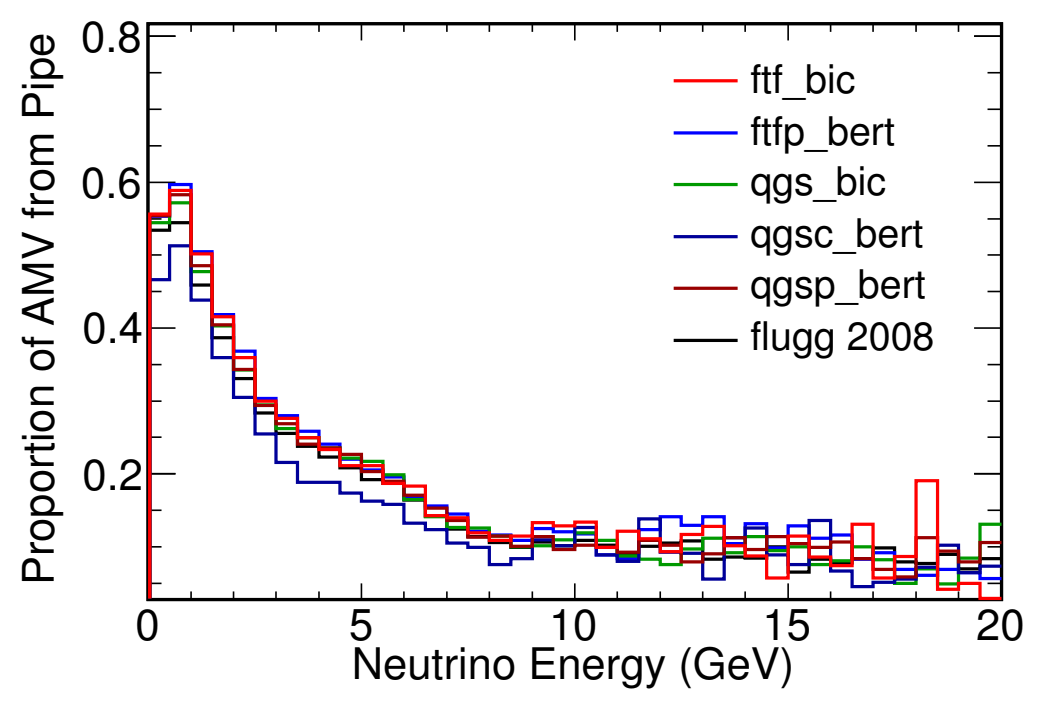

Figure 6.12: Comparison of the decay pipe component of the antineutrino flux in an FHC beam obtained with different physical models.

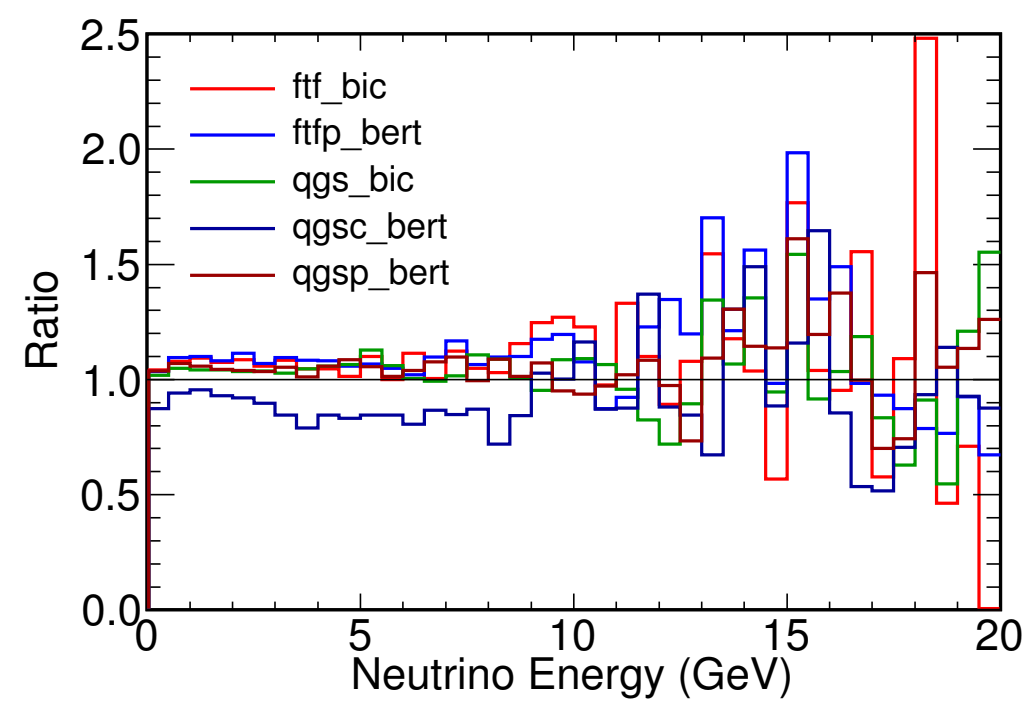

Figure 6.13: Ratio of the decay pipe component obtained with different models to that obtained from FLUGG. 
Since the difference between various models and FLUGG is less than $15 \%$ below $10 \mathrm{GeV}$ a maximum uncertainty of $15 \%$ was taken as the size of this systematic. This systematic is significant only for the antineutrino sample, and does not affect the neutrinos.

\subsubsection{Effect of Systematic Uncertainties}

The effect of systematic uncertainties is calculated by applying $\pm 1-\sigma$ systematic shifts to the simulated data with each systematic uncertainty. This gives two 'shifted' datasets corresponding to each systematic uncertainty considered. Combined fits to the shifted neutrino and antineutrino sets are performed and the shifted best fits are compared to the nominal (using unshifted Monte Carlo) best fit point. Since Lorentz and $\mathcal{C P} \mathcal{T}$ violating parameters are expected to be very small the systematic studies were done assuming the value of these parameters to be zero. The shifted datasets were obtained with $\Delta m^{2}=2.32 \times 10^{-3} \mathrm{eV}^{2}$, $\sin ^{2} 2 \theta=0.97, \tilde{g}_{\mu \bar{\mu}}^{Z T}=\tilde{g}_{\tau \bar{\tau}}^{Z T}=\left(c_{L}\right)_{\mu \mu}^{T T}=\left(c_{L}\right)_{\tau \tau}^{T T}=0$. Again, due to the degeneracy between the parameters, we fit the combination $\left(\tilde{g}_{\mu \bar{\mu}}^{Z T}-\tilde{g}_{\tau \bar{\tau}}^{Z T}\right)$ and $\left(\left(c_{L}\right)_{\mu \mu}^{T T}-\left(c_{L}\right)_{\tau \tau}^{T T}\right)$. Table 6.2 shows the shift in the value of SME parameters along with change in minimum likelihood for each systematically shifted dataset. The shifts from $\pm 1 \sigma$ shifted datasets have been added in quadrature to show the maximum possible shift. The significance of each systematic is quantified by the difference in the -2(log-likelihood) between the best nominal and shifted MC fits. The last row shows the 1- $\sigma$ statistical uncertainty. We can see from the table that the relative normalisation, muon energy scale, and hadronic energy scale are the dominant sources of systematic uncertainty. In order to take the effect of systematics into account the largest three systematics are included in the fit. These three systematics are correlated between neutrinos and antineutrinos. Fig. 6.14 shows the shift in best-fit $\tilde{g}_{\mu \bar{\mu}}^{Z T}$ and the change in sensitivity obtained by fitting the fake data shifted with $\pm 1 \sigma$ values of the largest three systematics. We can see that the systematic uncertainties are much smaller than the statistical uncertainty. Therefore, the results are not expected to be significantly affected due to systematic uncertainties. 


\begin{tabular}{lccc}
\hline Uncertainty & $\delta \tilde{g}$ & $\delta c$ & $\Delta(-2 \log \lambda)$ \\
\hline \hline Hadronic energy & $1.8 \mathrm{e}-27$ & $6.4 \mathrm{e}-24$ & 2.67 \\
Muon energy & $5.1 \mathrm{e}-27$ & $4.6 \mathrm{e}-24$ & 2.04 \\
Relative normalization & $5.9 \mathrm{e}-24$ & $7.3 \mathrm{e}-24$ & 0.60 \\
Beam flux & $-2.5 \mathrm{e}-24$ & $2.7 \mathrm{e}-24$ & 0.12 \\
Cross-sections & $1.5 \mathrm{e}-24$ & $5.3 \mathrm{e}-24$ & 0.06 \\
Decay pipe uncertainty & $9.3 \mathrm{e}-27$ & $2.4 \mathrm{e}-24$ & 0.03 \\
NC Contamination & $3.0 \mathrm{e}-32$ & $1.1 \mathrm{e}-24$ & 0.03 \\
WS Contamination & $1.6 \mathrm{e}-24$ & $1.5 \mathrm{e}-25$ & 0.01 \\
& & & \\
Statistical Error & $1.6 \mathrm{e}-23$ & $1.6 \mathrm{e}-23$ & 1.00 \\
\hline
\end{tabular}

Table 6.2: Shifts in the measurements of the parameters $\tilde{g}_{\mu \bar{\mu}}^{Z T}$ or $\tilde{g}_{\tau \bar{\tau}}^{Z T}$, and $\left(c_{L}\right)_{\mu \mu}^{T T}$ or $\left(c_{L}\right)_{\tau \tau}^{T T}$ between the unshifted and shifted best-fit points for each systematic. Also shown is the change in the value of $-2 \log \lambda$ at best-fit. (Input: $\Delta m^{2}=2.32 \times 10^{-3} \mathrm{eV}^{2}$, $\sin ^{2} 2 \theta=$ $0.97, \tilde{g} \equiv\left(\tilde{g}_{\mu \bar{\mu}}^{Z T}\right.$ or $\left.\left.\left.\tilde{g}_{\tau \bar{\tau}}^{Z T}\right)=0, c \equiv\left(\left(c_{L}\right)_{\mu \mu}^{T T} \operatorname{or}\left(c_{L}\right)_{\tau \tau}^{T T}\right)=0\right)\right)$. The last row shows the size of the statistical uncertainty.

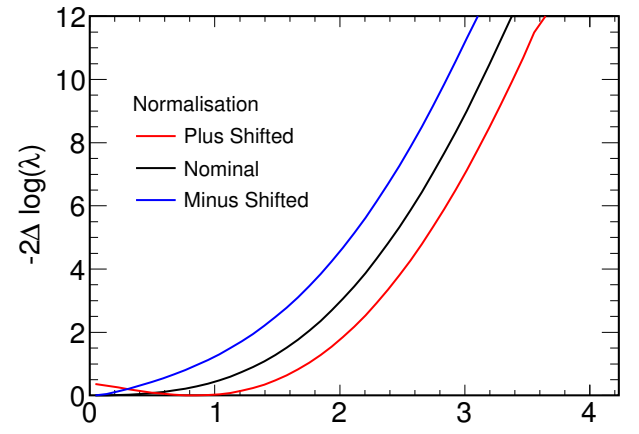

$|g|\left(\times 10^{-23}\right)$

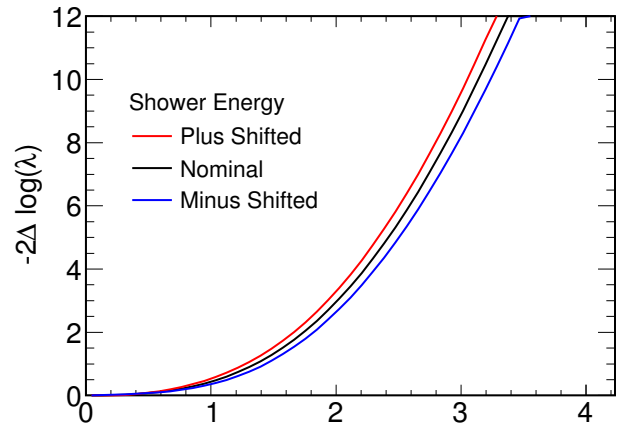

$\lg \mid\left(\times 10^{-23}\right)$

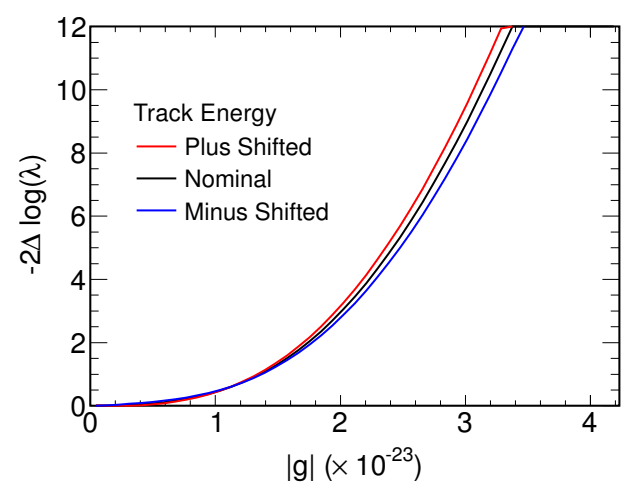

Figure 6.14: 1-D $\log$ (likelihood) plots showing the effect of the largest three systematics on $\mathrm{g} \equiv \tilde{g}_{\mu \bar{\mu}}^{Z T}$ when input $\tilde{g}_{\mu \bar{\mu}}^{Z T}=0$. All other parameters are marginalised. 


\subsection{Including Systematics in Fitting}

The fitting framework is similar to the one used in the NSI [150] and Decoherence analyses [151]. The log-likelihood method described earlier (Section 6.4) shows only the statistical uncertainty on the results. To take into account the effect of systematics on the result we include the three largest sources of systematic uncertainties in the fit as nuisance parameters. The systematics are assumed to have a Poisson distribution such that we can add a term

$$
\sum_{j=1}^{3} \frac{\epsilon_{j}^{2}}{\sigma_{j}^{2}}
$$

to the likelihood function Eq. 6.12, where $\epsilon_{j}$ corresponds to the shift from the nominal value of the $\mathrm{j}^{\text {th }}$ systematic and $\sigma_{j}$ is the uncertainty of the $\mathrm{j}^{\text {th }}$ systematic. The energy dependent systematic effects to be fitted are modelled by applying an energy dependent shift to the FD prediction based on the ratio of $1 \sigma$ shifted FD prediction and MC. Small differences are expected between $\mathrm{FD}$ predictions and $\mathrm{MC}$ even in the nominal case, therefore these differences are removed from the systematic error band by taking the ratio of nominal FD prediction and MC. A weight is defined in each energy bin as:

$$
w_{i}=\frac{M C_{i}^{\text {Shifted }}}{\operatorname{Pred}_{i}^{\text {Shifted }}} \times \frac{\operatorname{Pred}_{i}^{\text {Nominal }}}{M C_{i}^{\text {Nominal }}}-1
$$

The distribution of $w_{i}$ is then smoothed to reduce statistical fluctuation due to finite MC sets and interpolated to obtain a continuous function of weights $w(E)$, which are a function of reconstructed energy $E$. The smoothing is done using a LOWESS method [152] in which a linear fit is performed around each bin and the bin is set to the best-fit value. This helps us to capture important patterns in the data while leaving out statistical fluctuations. Since the uncertainties in different run periods are similar, the systematic ratios for the three run periods are combined to increase the MC statistics. For energy independent systematics, the function $w(E)$ is known to be constant and no binned distribution is needed. The distribu- 
tions of $w(E)$ for the absolute hadronic and track energy systematics are shown in Fig. 6.15, along with their corresponding smoothed distributions for neutrinos and antineutrinos. The very large fluctuations in case of antineutrinos is because of the fact that the antineutrino sample is statistically limited, but the smoothing procedure gives a reasonable estimate of the size of the error bands.
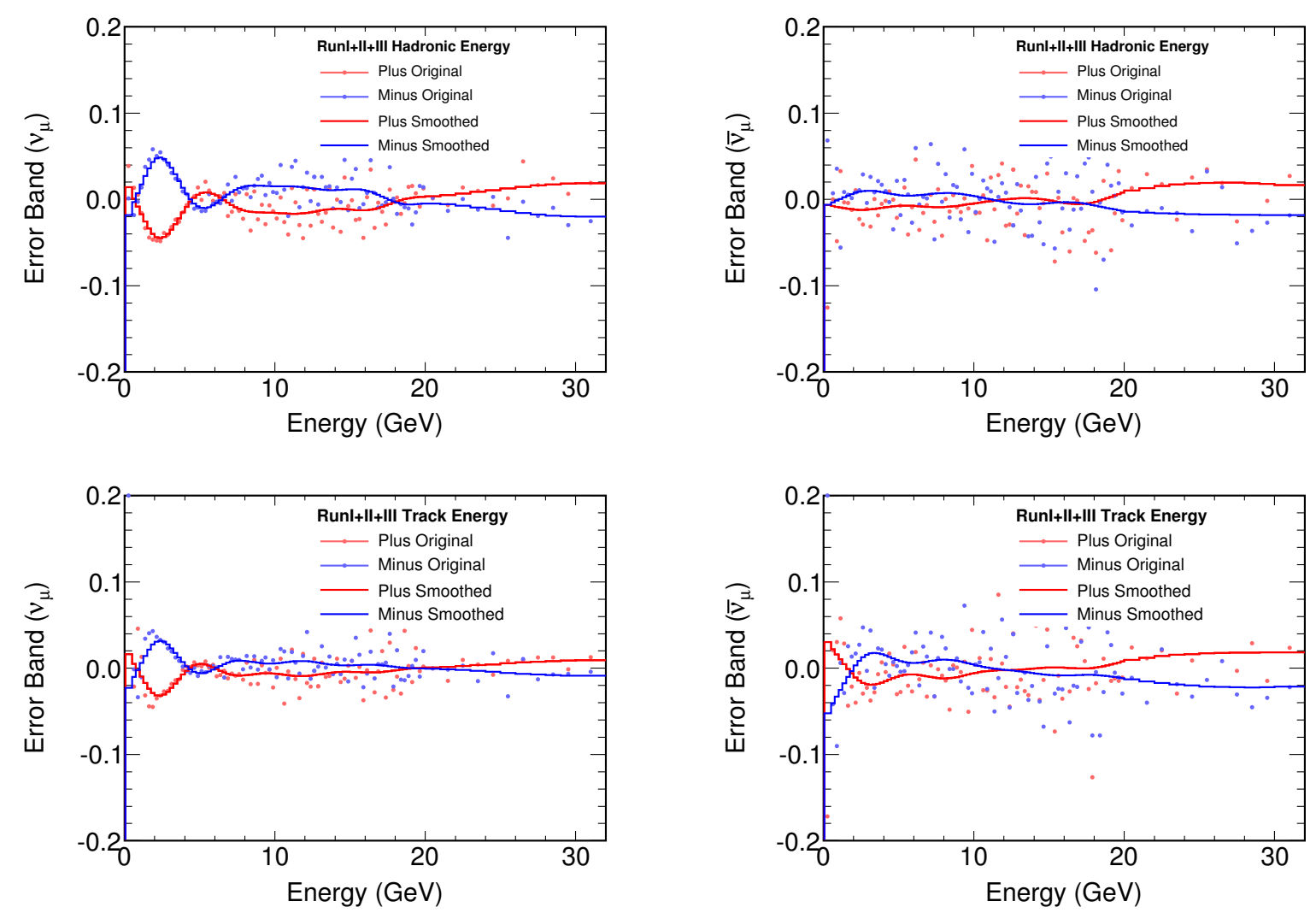

Figure 6.15: Error bands used in the modelling of systematic uncertainties for the neutrinos (left - top and bottom) and antineutrinos (right - top and bottom). The smoothed distributions are interpolated linearly to obtain a continuous function $\mathrm{w}(\mathrm{E})$ that is applied to shift the FD predictions.

The FD reconstructed energy spectrum is obtained from the ND data by the matrix method extrapolation. The best fit to data is calculated by minimising the log-likelihood 
function shown in equation 6.14 :

$$
-2 \log \lambda=2 \sum_{i=1}^{n}\left[P_{i}-D_{i}+D_{i} \ln \left(\frac{D_{i}}{P_{i}}\right)\right]+\sum_{j=1}^{3} \frac{\epsilon_{j}^{2}}{\sigma_{j}^{2}}
$$

where $\mathrm{P}_{i}$ is the expected number of events based on the FD prediction and $\mathrm{D}_{i}$ is the number of events from FD data in bin i.

The normalisation systematic is not energy dependent. Therefore, it is applied as a flat $4 \%$ systematic for antineutrinos and $1.3 \%$ for neutrinos. The penalty term is evaluated at every value of the parameters, marginalising over the separate systematic parameters. Allowing the systematics to float in the fit improves the goodness of fit and slightly increases the size of confidence levels.

\subsection{Sensitivity}

Using the fitting method as explained above and including the three largest systematics as nuisance parameters we can obtain the one-dimensional likelihood curves in $\tilde{g}_{\mu \bar{\mu}}^{Z T}, \tilde{g}_{\tau \bar{\tau}}^{Z T}$, $\left(c_{L}\right)_{\mu \mu}^{T T}$, and $\left(c_{L}\right)_{\tau \tau}^{T T}$. This is performed by marginalising over the parameters $\Delta m^{2}$ and $\sin ^{2} 2 \theta$, and fixing all other SME parameters to zero. Monte Carlo was generated with the input parameters $\Delta m^{2}=2.32 \times 10^{-3} \mathrm{eV}^{2}, \sin ^{2} 2 \theta=0.97$, and all the SME parameters set to zero. The red curve shows the statistics-only fits and the black curve shows the fits with the systematics included. Since $\tilde{g}_{\mu \bar{\mu}}^{Z T}$ and $\tilde{g}_{\tau \bar{\tau}}^{Z T}$ have identical sensitivity, we label them as $\tilde{g}_{\alpha \bar{\alpha}}^{Z T}$. From the figure it can be seen that the inclusion of systematics in the fit increases the size of the likelihood curve slightly without changing the best-fit point. So the systematics are expected to have very small effect on our measurement. From the sensitivity curves shown in Fig. 6.16 we observe that we can make a $3 \sigma$ measurement if the value of the parameters $\tilde{g}_{\alpha \bar{\alpha}}^{Z T}$ is greater than $3.4 \times 10^{-23}$ and that of $\left(c_{L}\right)_{\mu \mu}^{T T}$ and $\left(c_{L}\right)_{\tau \tau}^{T T}$ if they are greater than $6 \times 10^{-23}$. Fig. 6.17 shows what the FD antineutrino spectrum would look like at the $3 \sigma$ threshold of sensitivity to these parameters. 

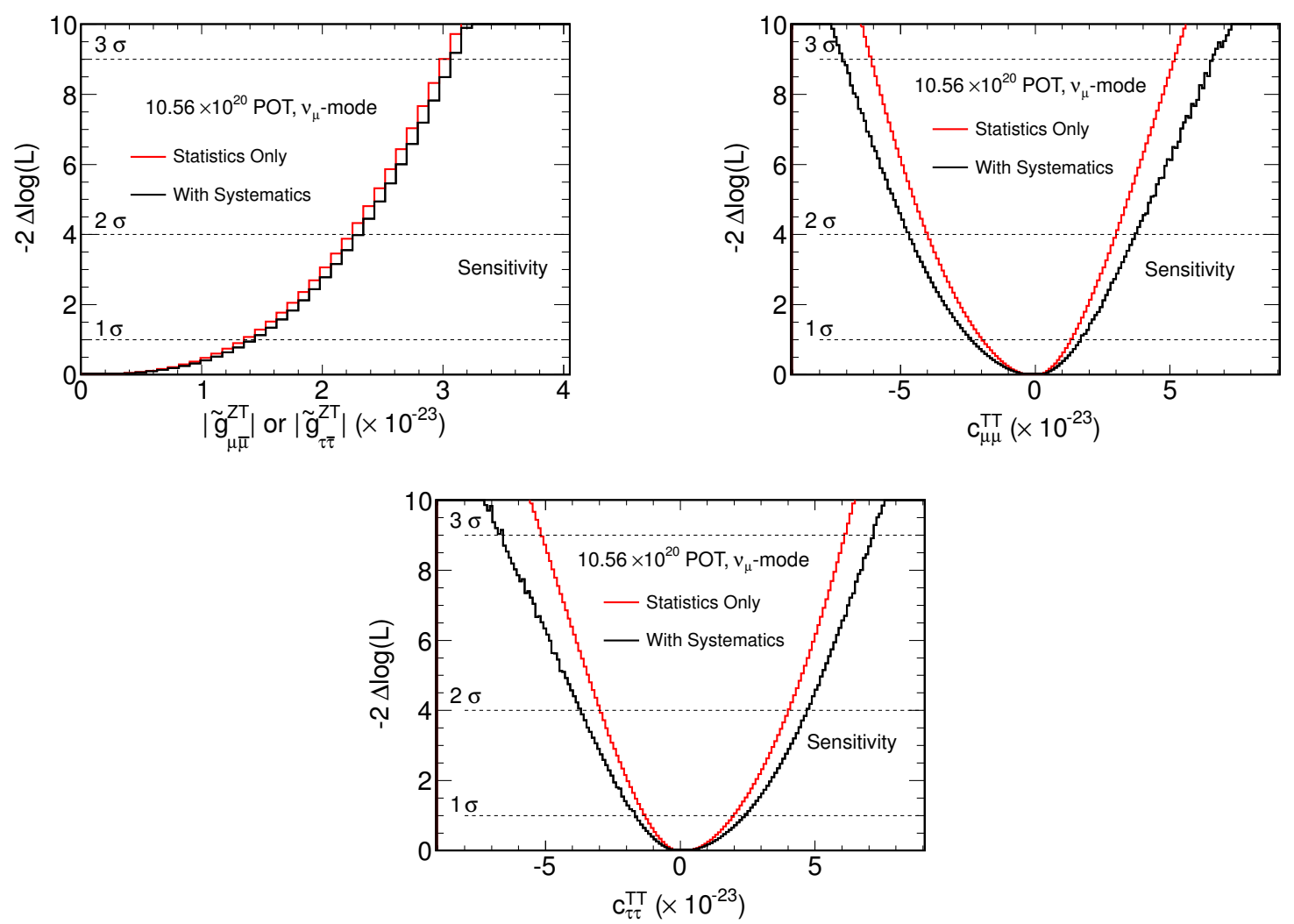

Figure 6.16: 1-dimensional $-2 \Delta \log \lambda$ distributions showing the fit to the parameters $\left|\tilde{g}_{\alpha \bar{\alpha}}^{Z T}\right|$, $\left(c_{L}\right)_{\mu \mu}^{T T}$, and $\left(c_{L}\right)_{\tau \tau}^{T T}$ for simulated data generated at $\tilde{g}_{\mu \bar{\mu}}^{Z T}=\tilde{g}_{\tau \bar{\tau}}^{Z T}=\left(c_{L}\right)_{\mu \mu}^{T T}=\left(c_{L}\right)_{\tau \tau}^{T T}=0$. The dashed lines show the 1- $\sigma, 2-\sigma$, and $3-\sigma$ sensitivities for the parameters. The statistics-only sensitivity is shown in red and that with the systematics included in the fit is shown in black.

\subsection{Results}

- The ND data is extrapolated using the beam matrix extrapolation and fitted for the individual SME parameters as explained above. The best-fit for the SME coefficients is very close to zero and at $3-\sigma$ confidence level we constrain

$$
\begin{gathered}
\left|\tilde{g}_{\mu \bar{\mu}}^{Z T}-\tilde{g}_{\tau \bar{\tau}}^{Z T}\right|<3.3 \times 10^{-23} \text {, and } \\
-8.4 \times 10^{-23}<\left(c_{L}\right)_{\mu \mu}^{T T}-\left(c_{L}\right)_{\tau \tau}^{T T}<8 \times 10^{-23} .
\end{gathered}
$$




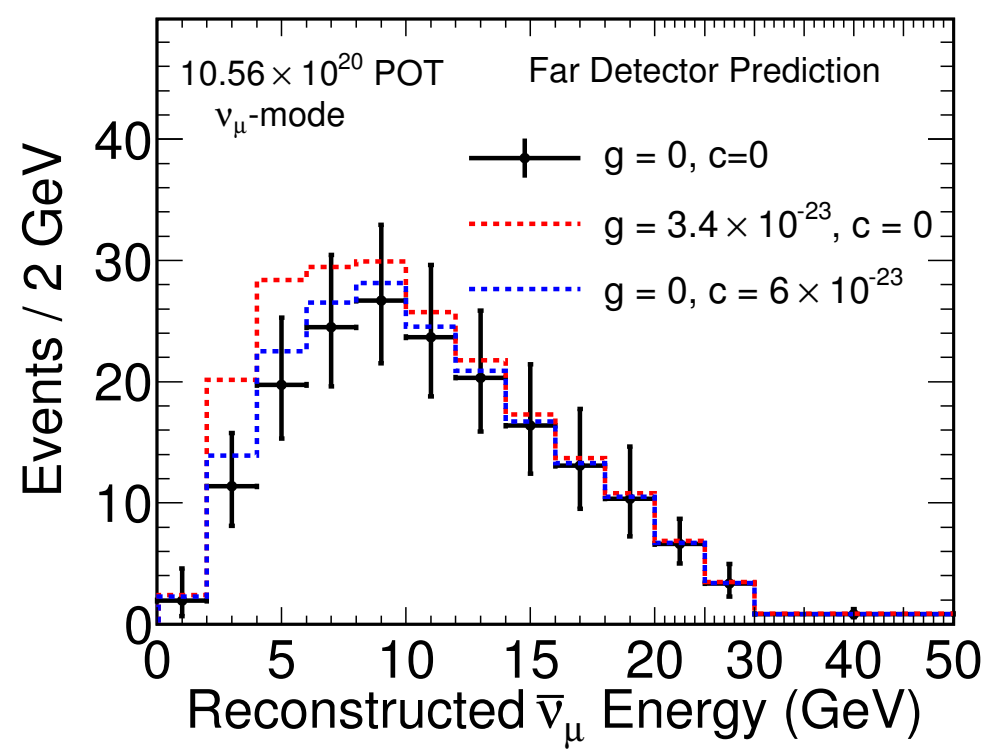

Figure 6.17: FD antineutrino spectrum expected at the 3- $\sigma$ threshold of sensitivity to $\mathrm{g}$ and c parameters, where $\mathrm{g} \equiv \tilde{g}_{\alpha \bar{\alpha}}^{Z T}$ and $\mathrm{c} \equiv\left(c_{L}\right)_{\mu \mu}^{T T}$ or $\left.\left(c_{L}\right)_{\tau \tau}^{T T}\right)$. The black dots show the spectrum when both $g$ and c-type SME coefficients are zero. The red histogram shows the FD spectrum predicted at $\tilde{g}_{\alpha \bar{\alpha}}^{Z T}=3.4 \times 10^{-23}$ and the blue histogram shows the FD prediction at $\left(c_{L}\right)_{\mu \mu}^{T T}=$ $6 \times 10^{-23}$.

as shown in Fig. 6.18. The values for $\Delta m^{2}$ and $\sin ^{2} 2 \theta$ at the best-fit are consistent with those in [97].

- We also obtain a two-dimensional likelihood contour obtained by simultaneously fitting $\tilde{g}_{\mu \bar{\mu}}^{Z T}-\tilde{g}_{\tau \bar{\tau}}^{Z T}$ and $\left(\left(c_{L}\right)_{\mu \mu}^{T T}-\left(c_{L}\right)_{\tau \tau}^{T T}\right) ; \Delta m^{2}$ and $\sin ^{2} 2 \theta$ are again marginalised. The contour in Fig. 6.19 shows the parameter space bound by these coefficients at 1- $\sigma, 2-\sigma$, and 3- $\sigma$ sensitivity.

The resulting FD simulated energy spectra, obtained by using the best fit values, are shown in Fig. 6.20 along with the neutrino and antineutrino spectra. If we plot the FD spectra expected using the 2-flavour oscillation model we can see that the spectra obtained using the best fit parameters from the SME model are almost indistinguishable from the 2-flavour prediction. So the data are consistent with no Lorentz and $\mathcal{C P} \mathcal{T}$ violation. We can tabulate the limits for the individual parameters as in shown Table. 6.3. These are first limits ever measured in these coefficients. 

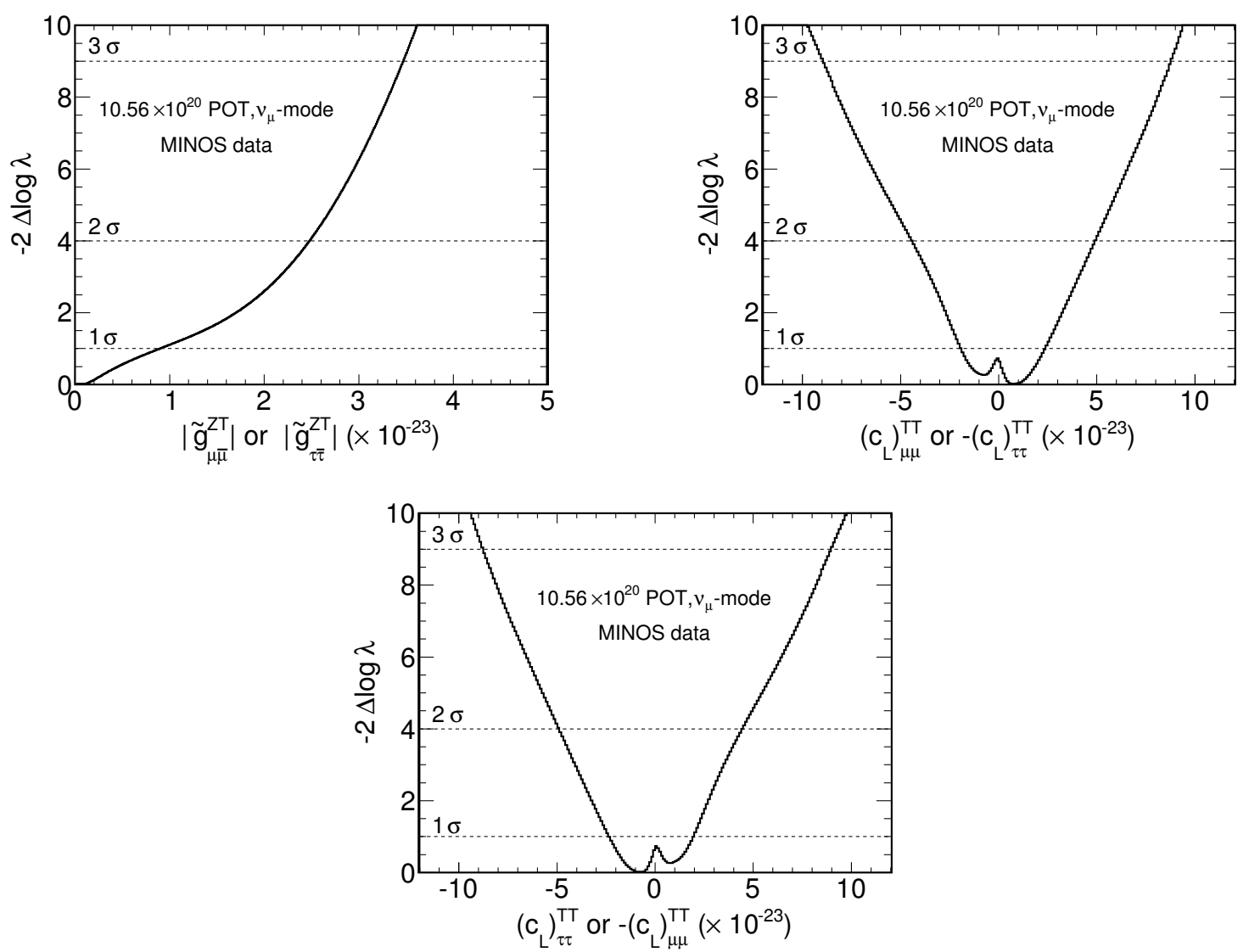

Figure 6.18: 1-dimensional $2 \Delta \log \lambda$ distributions showing the fit to the parameters $\left|\tilde{g}_{\alpha \bar{\alpha}}^{Z T}\right|$, $\left(c_{L}\right)_{\mu \mu}^{T T}$, and $\left(c_{L}\right)_{\tau \tau}^{T T}$. The dashed lines correspond to $1-\sigma, 2-\sigma$ and $3-\sigma$ confidence limits.

\begin{tabular}{lc}
\hline Coefficient & $3 \sigma$ Limit \\
\hline \hline$\left|\tilde{g}_{\mu \bar{u}}^{Z T}\right|$ & $<3.3 \times 10^{-23}$ \\
$\left|\tilde{g}_{\tau \bar{\tau}}^{Z T}\right|$ & $<3.3 \times 10^{-23}$ \\
$\left|\left(c_{L}\right)_{\mu \mu}^{T T}\right|$ or $\left|\left(c_{L}\right)_{\mu \mu}^{Z Z}\right|$ & $<8.4 \times 10^{-23}$ \\
$\left|\left(c_{L}\right)_{\tau \tau}^{T T}\right|$ or $\left|\left(c_{L}\right)_{\tau \tau}^{Z Z}\right|$ & $<8.4 \times 10^{-23}$ \\
\hline
\end{tabular}

Table 6.3: Table showing the constraints on the individual SME parameters. 


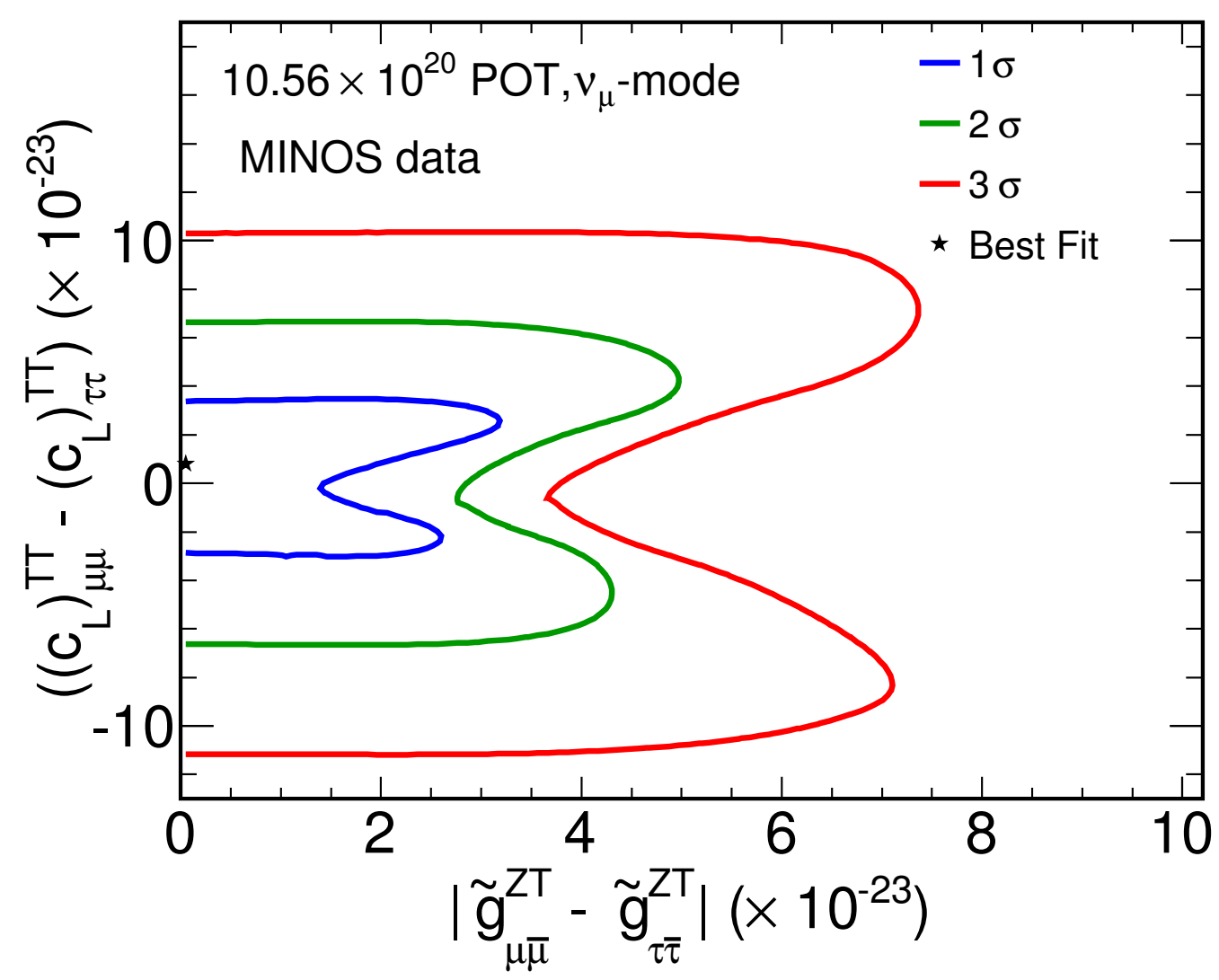

Figure 6.19: 2-dimensional $-2 \Delta \log \lambda$ distribution showing the fit to the parameters $\mid \tilde{g}_{\mu \bar{\mu}}^{Z T}-$ $\tilde{g}_{\tau \bar{\tau}}^{Z T} \mid$ and $\left(\left(c_{L}\right)_{\mu \mu}^{T T}-\left(c_{L}\right)_{\tau \tau}^{T T}\right)$. The blue, green and red contours show the 1- $\sigma, 2-\sigma$, and 3- $\sigma$ limits. 

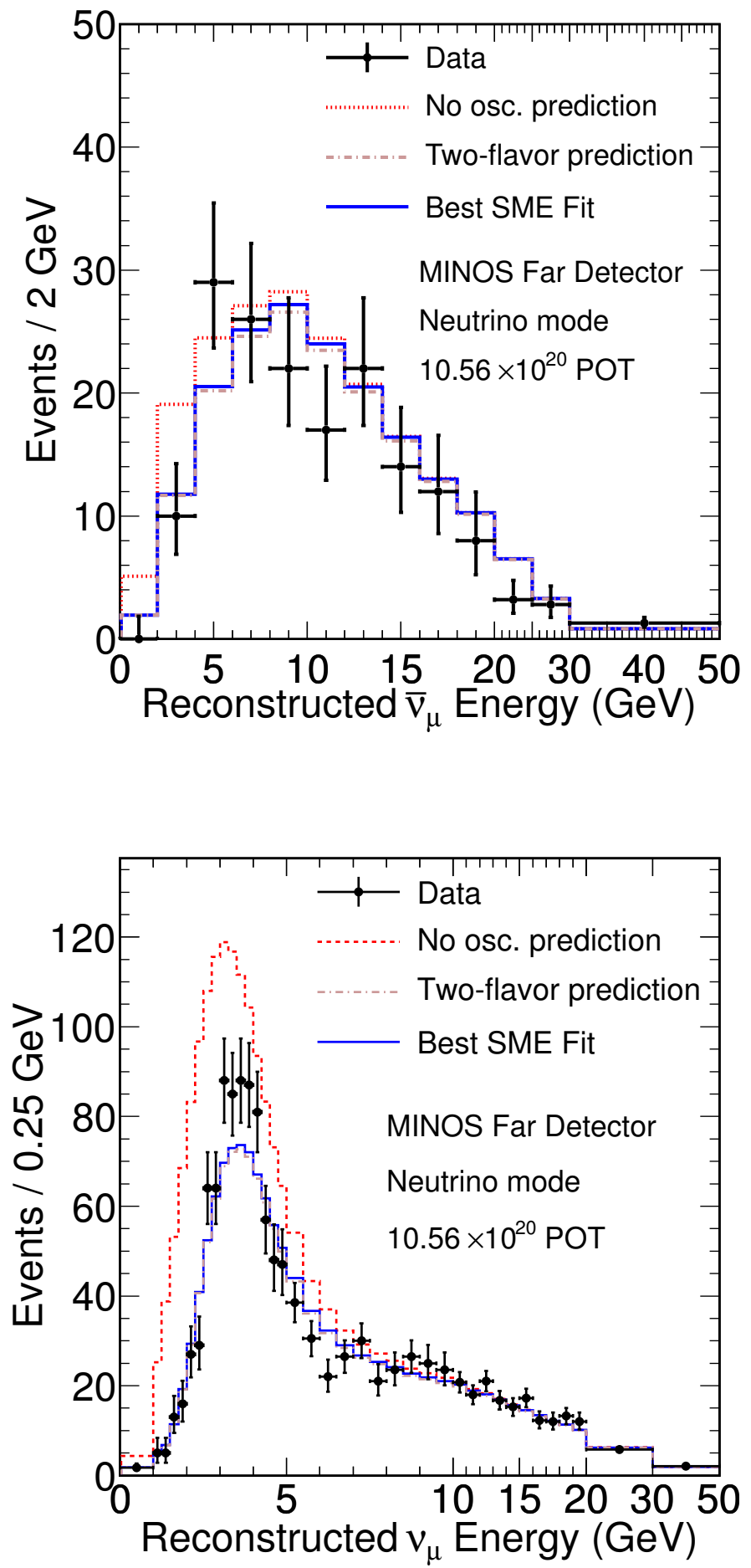

Figure 6.20: Far Detector distributions of selected antineutrino (left) and neutrino (right) events. Black dots represent data, the dashed histogram shows the prediction in the absence of oscillations, the dotted-dashed histogram shows the prediction at the values obtained in a fit to 2-flavour oscillation model, and the solid histogram shows the prediction at the values obtained in our fit to the SME model. The prediction obtained from a two flavour fit is very similar to that obtained from the SME fit. 


\section{Chapter 7}

\section{Summary and Outlook}

The new measurements presented in this thesis have added to the world's knowledge of neutrino oscillations. The measurement of antineutrino oscillation parameters from relatively high energy antineutrinos has helped in excluding all values of $\Delta \bar{m}_{32}^{2}>4.49 \times 10^{-3} \mathrm{eV}^{2}$. It also increased our confidence in our ability to select a small component of antineutrinos in the beam dominated by backgrounds. Data from the MINOS experiment have also been used to search for mixing between neutrinos and antineutrinos using a Lorentz and CPT violating formalism derived from SME. No sign of such a mixing has been observed and the data have been shown to be consistent with a 2-flavour prediction. This analysis helped obtain world's first limits on four time-independent SME coefficients.

MINOS finished data collection in its planned running in April, 2012. The detector performance has been very reliable in over seven years of beam data collection while the power of NuMI beam has continually increased. A total of $10.71 \times 10^{20}$ protons on target have been delivered in the neutrino-optimised mode, and $3.36 \times 10^{20}$ protons on target in the antineutrino-enhanced mode. Besides these MINOS has also collected 37.88 kt-years of atmospheric neutrino interactions. By combining all available data using the three-flavour formalism MINOS measured $\left|\Delta m_{32}^{2}\right|=[2.28-2.46] \times 10^{-3} \mathrm{eV}^{2}\left(68 \%\right.$ C.L.) and $\sin ^{2} \theta_{23}=$ $0.35-0.65$ (90\% C.L.) in the normal hierarchy, and $\left|\Delta m_{32}^{2}\right|=[2.32-2.53] \times 10^{-3} \mathrm{eV}^{2}$ 
(68\% C.L.) and $\sin ^{2} \theta_{23}=0.34-0.67$ (90\% C.L.) in the inverted hierarchy [153]. The resulting contours are shown in Fig. 7.1. The data show a marginal preference for inverted hierarchy [154]. MINOS constraints on $\Delta m^{2}$ are being used by the Daya Bay experiment in their measurement of $\theta_{13}$.

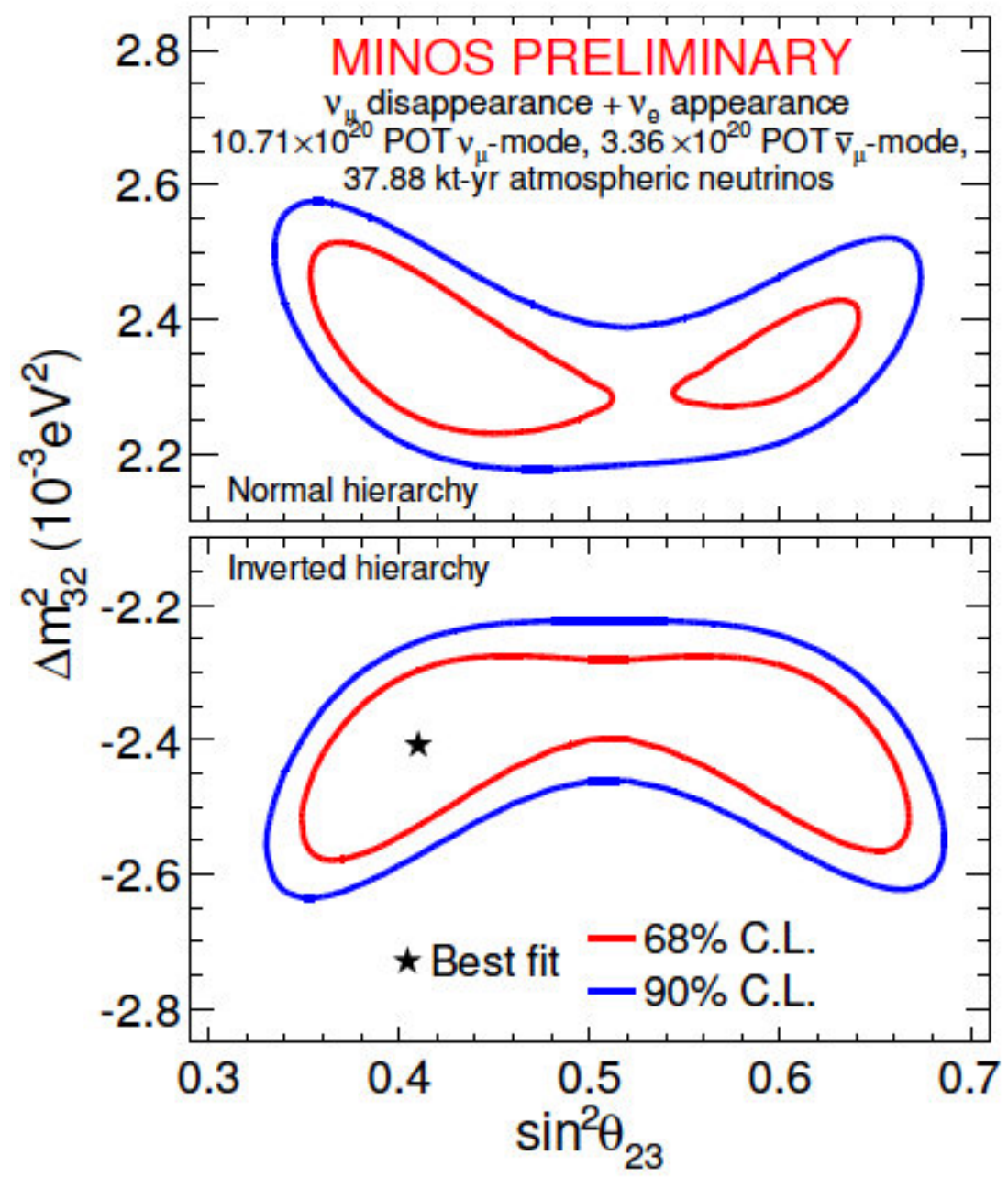

Figure 7.1: The $68 \%$ and $90 \%$ confidence limits for the parameters $\left|\Delta m_{32}^{2}\right|$ and $\sin ^{2} \theta_{23}$. Contours for both normal and inverted hierarchy are shown, and the $-\Delta \log L$ surface is calculated relative to the overall best-fit point. A marginal preference for inverted hierarchy and lower octant is shown.

The parameter $\theta_{13}$ was previously considered to be either zero or very small. With the new measurements confirming it to be relatively large it has opened up the possibility for a non-zero $\delta_{c p}$ and hence CP violation in the neutrino sector. Other important and challenging measurements that still need to be made are to determine the octant of $\theta_{23}$, resolve the 
mass hierarchy and to determine whether neutrinos are Dirac or Majorana particles. Long baseline experiments like $\mathrm{NO} \nu \mathrm{A}$ and $\mathrm{T} 2 \mathrm{~K}$ are capable of obtaining better constraints on mass hierarchy and $\delta_{c p}$. The NuMI beam has been upgraded to $700 \mathrm{~kW}$, delivering protons at higher intensity and higher energy. MINOS will continue running as MINOS+ and, along with $\mathrm{NO} \nu \mathrm{A}$ experiment, use this higher intensity beam to resolve the puzzle of mass hierarchy, $\delta_{c p}$ and the octant of atmospheric mixing parameters. There are ongoing experiments like CUORE [155] searching for neutrinoless double beta decay. The observation of such a decay would indicate that the neutrinos are Majorana particles. So far, no such signal has been observed.

Apart from the accelerator based experiments, experiments like the Indian Neutrino Observatory (INO) in Tamil Nadu, India, are also being built to study atmospheric neutrinos. The INO would be a magnetised iron calorimeter with Resistive Plate Chambers (RPCs) as the active detector. The physics capabilities of INO include resolution of mass hierarchy and determination of the octant of $\theta_{23}$.

There are ongoing searches for sterile neutrinos by MINOS [114]. This will be continued in MINOS+ and experiments like Microboone. The current $\nu \mathrm{SM}$ has also been extended to look for non standard interactions [156] or search for extra dimensions [157]. Search for Lorentz and CPT violation described in this thesis can be continued in the MINOS+ era as well since higher energy of the beam will make it more sensitive to lower value of the SME parameters, and stronger bounds can be obtained on them. Thus there is a huge potential for surprises in this field and the future of neutrino physics is very bright. 


\section{Appendix A}

\section{Light Level Studies for the Far}

\section{Detector}

\section{A.1 Introduction}

This chapter describes the attenuation study done for the FD. The aim of the study is to measure the change in light level obtained from the PMTs over a period of six years, between 2004 and 2010. Cosmic ray muons are used for measuring the amount of light incident on the PMTs. The scintillating light given out by the scintillator is carried by the wavelength shifting fibres to the end of the strips. The clear fibres then transport the light to the PMTs. The raw pulse height obtained from the PMTs is corrected for drifts, linearity and strip-to-strip variations. The present study uses the attenuation code [158] to obtain the attenuation curves.

\section{A.2 Average Pulse Height}

\section{A.2.1 Strip Selection}

As described in detail in Section 3.2 .2 the FD consists of 486 octagonal steel planes. Out of these only 484 planes are instrumented. The arrangement of the strips in the FD are 
shown in Fig. 3.11. Only the $8 \mathrm{~m}$ strips in the FD are used for this study numbered from 56 to 136 . This is because they are the longest strips in the detector and give a better response to the light incident on them. The strips numbered 90 to 101 are removed since they pass near the coil hole through the centre of the detector. The pulse height obtained from the attenuation code is plotted as a function of the longitudinal track position along the strip. It has been observed in the previous studies [105] that the light attenuation in fibres has two components - a component with long attenuation length and the other with short attenuation length. Thus the attenuation curves can be fitted quite well with a sum of two exponentials $A e^{-k_{1} x}+B e^{-k_{2} x}$, where $\mathrm{x}$ is the length along the strip and $k_{1}$ and $k_{2}$ are inverse of attenuation lengths. A fit was performed for each strip and only the strips with $\chi^{2} / n d f$ between 0.7 and 2 to a fit to this function were selected. Same strips were selected for the years 2004, 2007 and 2010.

\section{A.2.2 Procedure}

The pulse height for all the $8 \mathrm{~m}$ strips in all the planes is averaged using $20 \mathrm{~cm}$ bins of distance along strip to obtain the average pulse height. MINOS FD is read out from both ends, also called East and West ends, for all scintillator strips oriented along U and $\mathrm{V}$ directions. So the averaging is done separately for U East, U West, V East and V West readouts. Then the attenuation plots from $\mathrm{U}$ West and $\mathrm{V}$ West strips are inverted and the four views are combined together to obtain the average pulse height. This gives us the average pulse height for the whole detector as a function of distance from the readout end. Figures A.1 and A.2 show the average pulse height for the years 2004, 2007 and 2010.

\section{A.3 Pulse Height Ratios}

The average pulse height histograms are used to calculate the pulse height ratio for the years 2010/2004, 2010/2007 and 2007/2004, as shown by figure A.3. The ratios are normalised to have an average value of one. The dip observed in the middle shows that more 


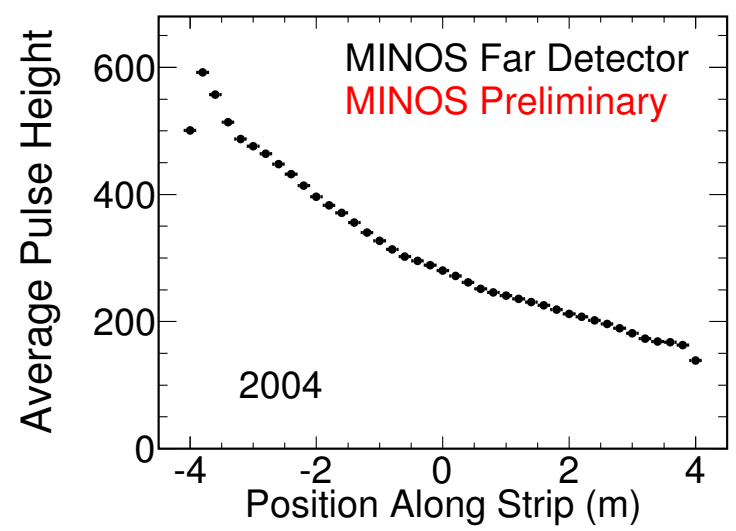

Figure A.1: Average light output as a function of distance from the readout end for the year 2004. The light output from east and west ends for U-view and V-view strips have been combined.
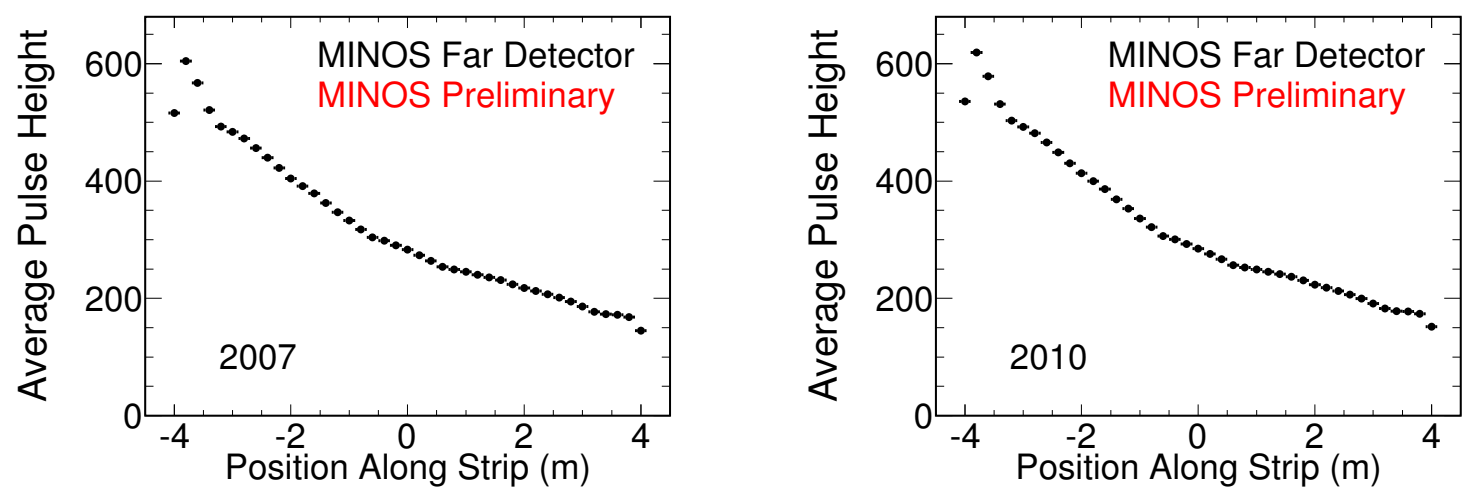

Figure A.2: Average light output as a function of distance from the readout end for the years 2007(left) and 2010. The light output from east and west ends for U-view and V-view strips have been combined.

light is lost at the centre of the strips as compared to that at the ends.

Furthermore, from the pulse height ratio at a distance of $-3 \mathrm{~m}$ and $3 \mathrm{~m}$ from the centre of the strip, we can calculate the change in light across the strips. Table A.1 shows the change in the light level for U East, U West, V East and V West strips separately. After combining the different views together, the change in the light level was calculated to be approximately $1.8 \%$ over a distance of $6 \mathrm{~m}$ between the years 2004 and 2010. 


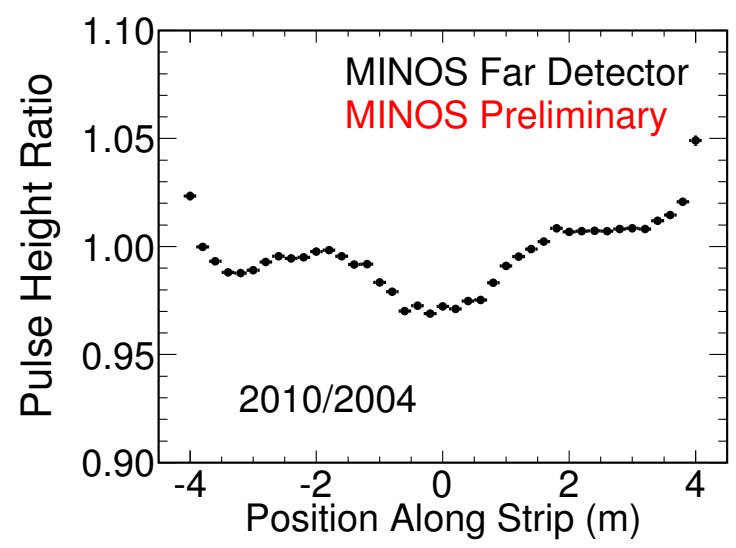

Figure A.3: Ratio of average light output in the year 2010 to that in 2004 as a function of distance from the readout end.

\begin{tabular}{|c|c|c|c|c|c|}
\hline Years & U East & U West & V East & V West & Average \\
\hline \hline 2010 to 2004 & $2.192 \%$ & $1.440 \%$ & $1.156 \%$ & $2.395 \%$ & $1.797 \pm 0.641 \%$ \\
\hline 2010 to 2007 & $1.426 \%$ & $0.599 \%$ & $0.524 \%$ & $1.144 \%$ & $0.923 \pm 0.503 \%$ \\
\hline 2007 to 2004 & $1.312 \%$ & $0.847 \%$ & $0.636 \%$ & $1.264 \%$ & $0.882 \pm 0.430 \%$ \\
\hline
\end{tabular}

Table A.1: Change in light level over $6 \mathrm{~m}$. The change is calculated from the pulse height ratio at $3 \mathrm{~m}$ and $-3 \mathrm{~m}$ from the centre.

\section{A.4 Attenuation Lengths}

In order to obtain the change in attenuation lengths over time, the total pulse height histogram is fitted with the function $A e^{-k_{1} x}+B e^{-k_{2} x}$. The region from $-2 \mathrm{~m}$ to $2 \mathrm{~m}$ from the centre of the strip is removed while fitting to exclude the region with the dip. The figures A.4, A.5 and A.6 show the fitted curves along with the reduced fit parameters. The fit parameters indicate that the attenuation in the fibres is dominated by the component with longer attenuation length, and that the longer attenuation lengths increase over time.

\section{A.5 Light level at the Centre of the Detector}

Since the light level is seen to be changing more rapidly at the centre of the detector it is useful to quantify the total change at the centre relative to the outer edge of the detector. All the strips in the FD are selected for this study. The pulse height in each strip at a particular 


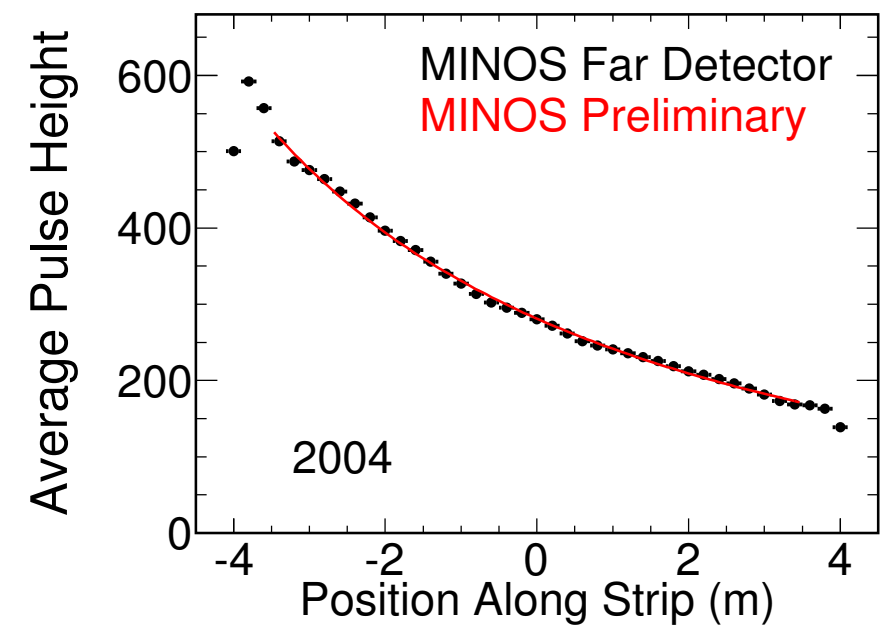

Figure A.4: Average light output as a function of distance from the readout end for the year 2004. The light output is fitted to a function of the form $A e^{-k_{1} x}+B e^{-k_{2} x}$. The fit parameters are $A=250.87, B=30.18, k_{1}=0.12 \times 10^{-2}, k_{2}=0.45$. After removing the region between $-2 \mathrm{~m}$ and $2 \mathrm{~m}$ the reduced fit parameters are $A=294.09, B=8.27 \times 10^{-12}$, $k_{1}=1.606 \times 10^{-1}, k_{2}=3.239 \times 10^{-1}$

position in a plane is averaged separately over all planes thus obtaining the attenuation curves as a function of strip number in the plane. The pulse height ratio for the years 2010/2004, 2010/2007 and 2007/2004 is plotted as a function of strip number and distance along the strip. To cancel the strip-to-strip variations, the ratio in each strip is normalised to have an average value of 1 at the centre of the strip. The pulse heights from U East and $\mathrm{U}$ West readouts are added together to obtain the average pulse height ratio as a function of strip number for all U strips. Similarly, the pulse heights from V East and V West readouts are added to obtain the average pulse height ratio as a function of strip number for all $\mathrm{V}$ strips. The $\mathrm{U}$ and $\mathrm{V}$ views are plotted separately, as shown in figure A.7.

In order to combine the $\mathrm{U}$ and the $\mathrm{V}$ views, the strips in $\mathrm{V}$ view are rotated by 90 degrees and then added to the strips in $U$ view. This ensures that all the strips map the same spatial region of the detector. Figure A.8 shows the combined histogram. The light loss at the centre of the detector is quantified by plotting the pulse height ratio as a function of radial position from the centre. Figure A.9 shows the pulse height ratio for the years 2010/2004. 


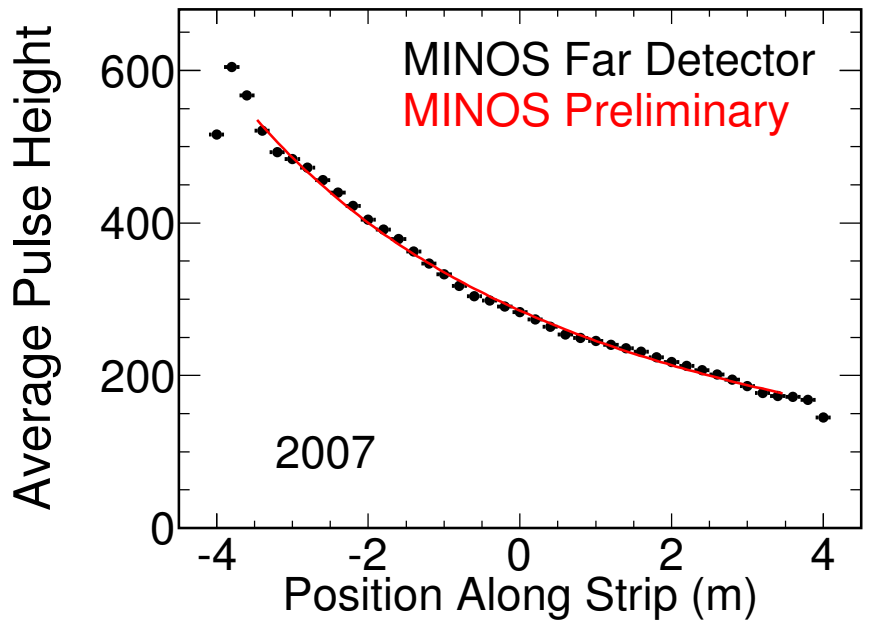

Figure A.5: Average light output as a function of distance from the readout end for the year 2007. The light output is fitted to a function of the form $A e^{-k_{1} x}+B e^{-k_{2} x}$. The fit parameters are $A=230.39, B=54.69, k_{1}=0.10 \times 10^{-2}, k_{2}=0.385$. After removing the region between $-2 \mathrm{~m}$ and $2 \mathrm{~m}$ the reduced fit parameters are $A=300.31, B=0.42 \times 10^{-12}$, $k_{1}=1.592 \times 10^{-1}, k_{2}=3.852 \times 10^{-1}$

It can be described reasonably well by a gaussian, and the light level at the centre of the detector is observed to be approximately $2.6 \%$ lower than at the ends.

\section{A.6 Summary}

From the present study the light level obtained from the PMTs in the FD are observed to change at a different rate in different parts of the detector. Overall, the increase in light level is $1.8 \%$ in 6 years. It has also been observed that the centre of the strips are aging more quickly than the edges. The difference in light level between the centre of the detector and the edges is $2.6 \%$. The reason for these observations is not clearly understood yet. A hypothesis is that mechanical stresses in the scintillator could cause the light level to change at different rates. More studies are underway to investigate the aging of the strips in the FD. 


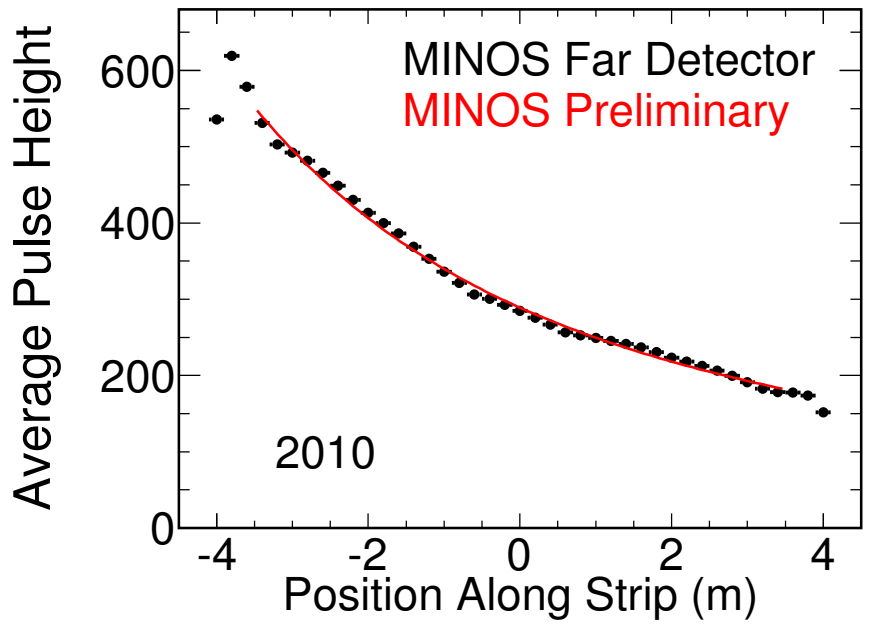

Figure A.6: Average light output as a function of distance from the readout end for the year 2010. The light output is fitted to a function of the form $A e^{-k_{1} x}+B e^{-k_{2} x}$. The fit parameters are $A=226.06, B=62.85, k_{1}=8.9 \times 10^{-2}, k_{2}=0.387$. After removing the region between $-2 \mathrm{~m}$ and $2 \mathrm{~m}$ the reduced fit parameters are $A=307.49, B=85.8 \times 10^{-12}$, $k_{1}=1.577 \times 10^{-1}, k_{2}=3.066 \times 10^{-1}$
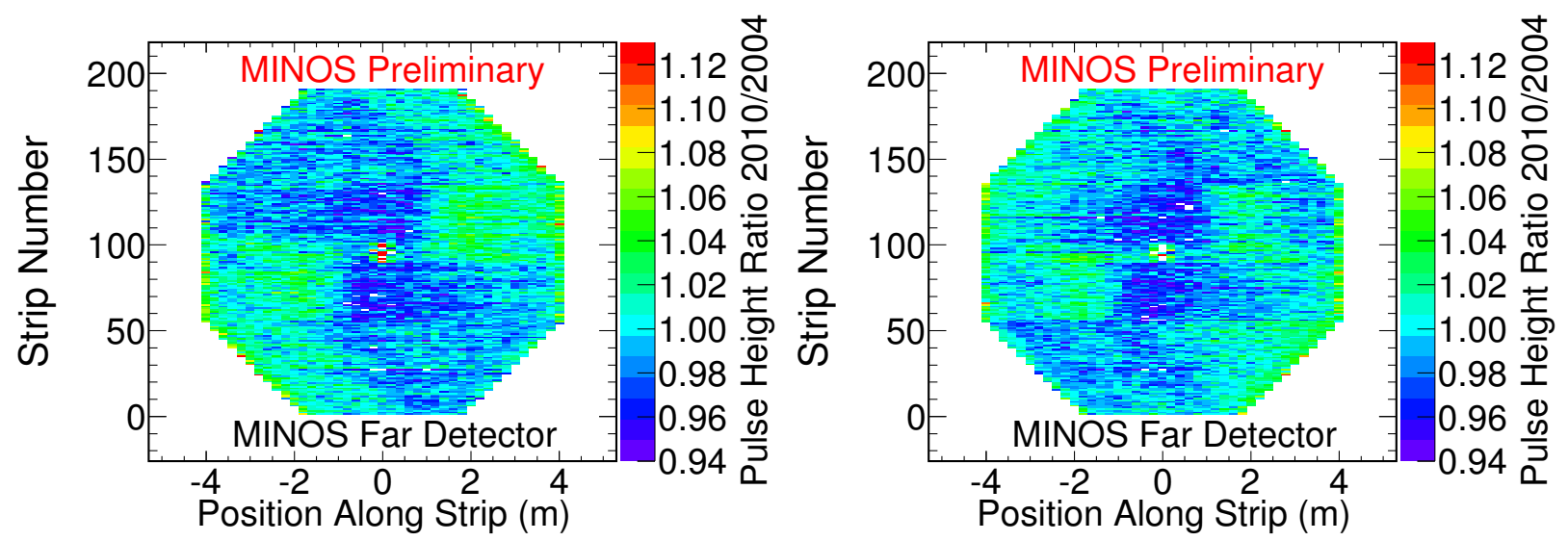

Figure A.7: Ratio of average light output in the year 2010 to that in 2004 as a function of distance from the centre of the strips. (Left) The light output from the east and the west ends for strips in U-view have been combined, (Right) The light output from the east and the west ends for strips in U-view have been combined. 


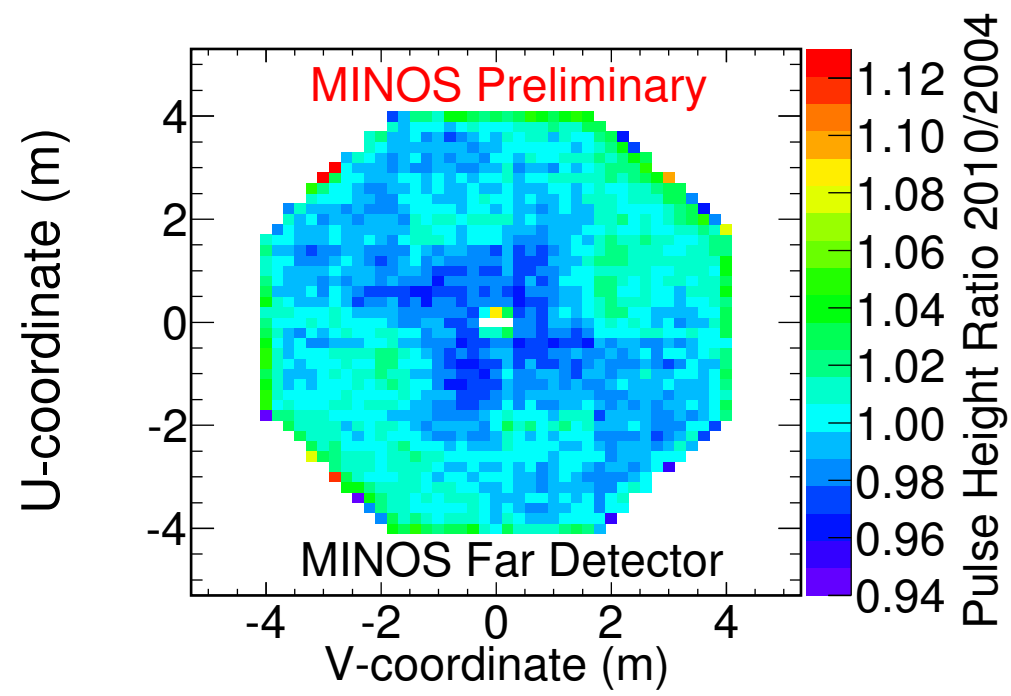

Figure A.8: Ratio of average light output in 2010 and 2004 as a function of distance from the centre of strips. The output from east and west readout ends of strips in both U-view and $\mathrm{V}$-view have been combined.

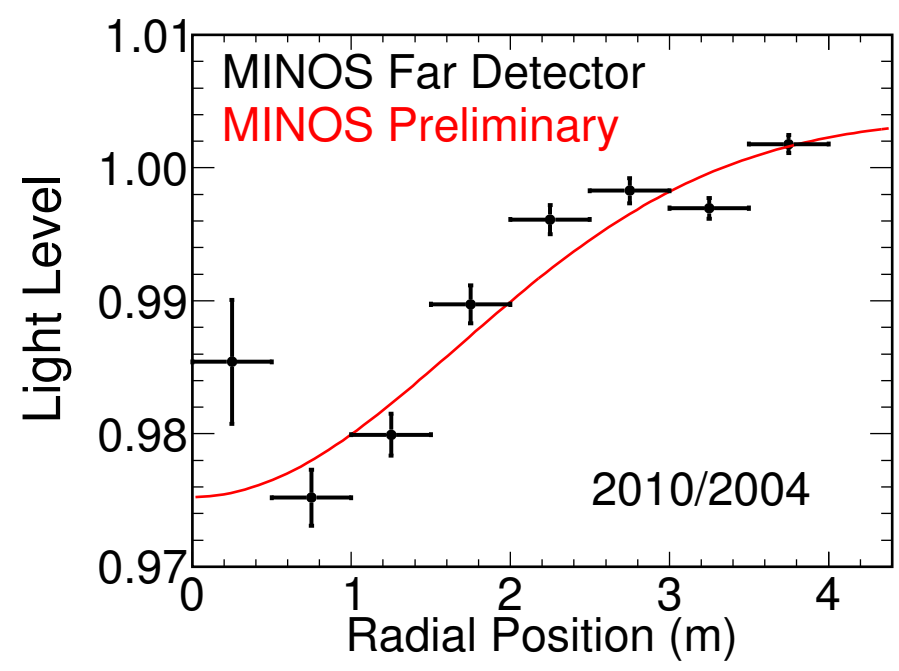

Figure A.9: Ratio of light output in 2010 to 2004 as a function of radial distance from the centre of the detector. The light level at the centre changes by $2.6 \%$ w.r.t the average. 


\section{References}

[1] Wolfgang Pauli, "Letter to the physical society of Tubingen," 1930.

[2] Y. Fukuda et. al., (Super-Kamiokande Collab.), Phys. Rev. Lett., vol. 81, pp. 1562, 1998.

[3] O. Hahn and L. Meitner, "Uber die Absorption der $\beta$-Strahlen einiger Radioelemente," Physikalische Zeitschrift, vol. 9, pp. 321, 1908.

[4] O. von Baeyer, O. Hahn, and L. Meitner, "Nachweis von $\beta$-Strahlen bei Radium D," Physikalische Zeitschrift, vol. 12, pp. 378, 1911.

[5] J. Chadwick, "Distribution in intensity in the magnetic spectrum of the $\beta$-rays of radium," Ver. Dtsh. Physik. Ges., vol. 16, pp. 383-391, 1914.

[6] C. D. Ellis and W. A. Wooster, "The average energy of disintegration of radium E," Proc. of the Royal Soceity of London: A, vol. 117, pp. 109-123, 1927.

[7] J. Chadwick, "Possible existence of a neutron," Nature, vol. 129, pp. 312, 1932.

[8] D. Iwanenko, "The Neutron Hypothesis," Nature, vol 129, pp. 798, 1932.

[9] W. Heisenberg, "Uberden den Bau der Atomkerne. I.," Zeitschrift fur Physik, vol. 77, pp. 1, 1932.

[10] W. Heisenberg, "Uberden den Bau der Atomkerne. II.," Zeitschrift fur Physik, vol. 78, pp. $156,1932$. 
[11] W. Heisenberg, "Uberden den Bau der Atomkerne. III.," Zeitschrift fur Physik, vol. 80, pp. 587, 1933.

[12] F. Perrin, "Possibilite d'emission de particules neutres de masse intrinseque nulle dans les radiactivites," Comptes Rendus des Seances de l'Academie des Sciences, vol. 197, pp. 1625, 1933.

[13] E. Fermi, "An attempt of a theory of beta radiation," Z. Phys., vol. 88, pp. 161-177, 1934.

[14] F. L. Wilson, "Fermi's Theory of Beta Decay," American Journal of Physics, vol. 36, pp. 161, 1934.

[15] G. Gamow and E. Teller, "Selection rules for the $\beta$-disintegration," Phys. Rev., vol. 49, pp. 895, 1936.

[16] H. Bethe and R. Peierls, "The 'neutrino'," Nature, vol. 133, pp. 532, 1934.

[17] F. Reines and C. Cowan, "Detection of the Free Neutrino," Phys. Rev., vol. 92, pp. 830, 1953.

[18] F. Reines and C. Cowan, "A Proposed Experiment to Detect the Free Neutrino," Phys. Rev., vol. 90, pp. 492, 1953.

[19] F. Reines, C. Cowan, F. Harrison, E. Anderson, and F. Hayes, "Large Liquid Scintillation Detectors," Phys. Rev., vol. 90, pp. 493, 1953.

[20] F. Reines and C. Cowan, "The Neutrino," Nature, vol. 178, pp. 446, 1956.

[21] C. L. Cowan Jr., F. Reines, et. al., "Detection of the free neutrino: a confirmation," Science, vol. 124, pp. 103-104, 1956.

[22] F. Reines, C. Cowan, F. Harrison, A. McGuire, and H. Kruse, "Detection of the Free Antineutrino," Phys. Rev., vol. 117, pp. 159, 1960. 
[23] C. S. Wu, E. Ambler, R. W. Hayward, D. D. Hoppes, and R. P. Hudson, "Experimental Test of Parity Conservation in Beta Decay," Phys. Rev., vol. 105, pp. 1413, 1957.

[24] R. P. Feynman and M. Gell-Mann, "Theory of the Fermi Interaction," Phys. Rev., vol. 109, pp. 193, 1958.

[25] E. C. G. Sudarshan and R. E. Marshak, "Chirality Invariance and the Universal Fermi Interaction," Phys. Rev., vol. 109, pp. 1860, 1958.

[26] E. D. Commins and P. H. Bucksbaum, "Weak Interactions of Leptons and Quarks," Cambridge University Press, 1983.

[27] W. Heisenberg, "Zur Theorie der Schauer in der Hohenstrahlung," Zeitschrift fur Physik, vol. 101, pp. 533, 1936.

[28] Sheldon L. Glashow, "Partial-symmetries of weak interactions," Nucl. Phys., vol. 22, pp. 579-588, 1961.

[29] Steven Weinberg, "A model of leptons," Phys. Rev. Lett., vol. 19, pp. 1264-1266, 1967.

[30] A. Salam and J. C. Ward, "Electromagnetic and weak interactions," Phys. Lett., vol. 13, pp. 168-171, 1964.

[31] F. J. Hasert et. al. (Gargamelle Neutrino Collaboration), "Search for elastic muon neutrino electron scattering," Phys. Lett. B, vol. 46, pp. 121, 1973.

[32] F. J. Hasert et. al. (Gargamelle Neutrino Collaboration), "Observation of neutrino-like interactions without muon or electron in the Gargamelle neutrino experiment," Phys. Lett. B, vol. 46, pp. 138, 1973.

[33] G. Arnison et. al. (UA1 Collaboration), "Experimental Observation of Isolated Large Transverse Energy Electrons With Associated Missing Energy at $\sqrt{s}=540 \mathrm{GeV}$," Phys. Lett. B, vol. 122, pp. 103, 1983. 
[34] R. Banner et. al. (UA2 Collaboration), "Observation of Single Isolated Electrons of High Transverse Momentum in Events with Missing Transverse Energy at the CERN $\bar{p} p$ Collider," Phys. Lett. B, vol. 122, pp. 476, 1983.

[35] G. Arnison et. al. (UA1 Collaboration), "Experimental observation of lepton pairs of invariant mass around $95 \mathrm{GeV} / \mathrm{c}^{2}$ at the CERN SPS collider," Phys. Lett. B, vol. 126, pp. 398, 1983.

[36] P. Bagnaia et. al. (UA2 Collaboration), "Evidence for $Z^{0} \rightarrow e^{+} e^{-}$at the CERN anti-p p collider," Phys. Lett. B, vol. 129, pp. 130, 1983.

[37] G. Danby et. al., "Observation of high-energy neutrino reactions and the existence of two kinds of neutrinos," Phys. Rev. Lett., vol. 9, pp 36-44, 1962.

[38] Martin L. Perl, G.S. Abrams, A. Boyarski, Martin Breidenbach, D. Briggs, et. al., "Evidence for Anomalous Lepton Production in e+ - e- Annihilation," Phys. Rev. Lett., vol. 35, pp. 1489-1492, 1975.

[39] S. Schael et. al., "Precision electroweak measurements on the Z resonance," Phys. Rept., vol. 427, pp. 257-454, 2006.

[40] K. Kodama et. al. (DONUT Collaboration), "Observation of Tau Neutrino Interactions," Phys. Lett. B, vol. 504, pp. 218, 2001.

[41] K. Kodama et. al., "Final tau-neutrino results from the DONUT experiment," Phys. Rev. D, vol. 78, pp. 052002, 2008.

[42] J. Beringer et. al. (Particle Data Group), Phys. Rev. D, vol. 86, pp. 010001, 2012.

[43] ATLAS Collaboration, "Observation of a new particle in the search for the Standard Model Higgs boson with the ATLAS detector at the LHC," Phys. Lett. B, vol. 716, pp. 1, 2012. 
[44] CMS Collaboration, "Observation of a new boson at a mass of $125 \mathrm{GeV}$ with the CMS experiment at the LHC," Phys. Lett. B, vol. 716, pp. 30, 2012.

[45] Peter W. Higgs, "Spontaneous symmetry breakdown without massless bosons," Phys. Rev., vol. 145(4), pp.1156-1163, 1966.

[46] B. Pontecorvo, "Mesonium and antimesonium," Sov. Phys. JETP, vol. 6, pp. 429, 1957.

[47] B. Pontecorvo, "Inverse beta processes and nonconservation of lepton charge, Soviet Physics-JETP, vol.7, pp. 172-173, 1958.

[48] M. Gell-Mann and A. Pais, "Behavior of neutral particles under charge conjugation, Physical Review, vol. 97, pp. 1387-1389, 1955.

[49] Z. Maki, M. Nakagawa, and S. Sakata, "Remarks on the Unified Model of Elementary Particles," Progress of Theoretical Physics, vol. 28, pp. 870, 1962.

[50] W. M. Yao et. al., "Review of Particle Physics," Journal of Physics G: Nuclear and Particle Physics, vol. 33, pp. 1, 2006.

[51] L. Wolfenstein, "Neutrino oscillations in matter," Phys. Rev. D, vol. 17, pp. 23692374, May 1978.

[52] S. Mikheev and A. Smirnov, "Resonance Amplification of Oscillations in Matter and Spectroscopy of Solar Neutrinos," Sov. J. Nucl. Phys., vol. 42, pp. 913-917, 1985.

[53] J. Davis, Raymond, D. S. Harmer, and K. C. Hoffman, "Search for neutrinos from the sun," Phys. Rev. Lett., vol. 20, pp. 1205-1209,1968.

[54] John N. Bahcall, Aldo M. Serenelli, and Sarbani Basu, "New solar opacities, abundances, helioseismology, and neutrino fuxes," Astrophys. J., vol. 621, L85-L88, 2005. 
[55] K. Hirata et. al., "Observation of B-8 Solar Neutrinos in the Kamiokande-II Detector," Phys. Rev. Lett., vol. 63, p. 16, 1989.

[56] A. Abazov, O. Anosov, E. Faizov, V. Gavrin, A. Kalikhov, et. al., "Search for neutrinos from sun using the reaction Ga-71 (electron-neutrino e-) Ge-71," Phys. Rev. Lett., vol. 67, pp. 3332-3335, 1991.

[57] P. Anselmann et. al., "Solar neutrinos observed by GALLEX at Gran Sasso.," Phys. Lett. B, vol. 285, pp. 376-389, 1992.

[58] J. Boger et. al., "The Sudbury neutrino observatory," Nucl. Instrum. Meth. A, vol. 449, pp. 172-207, 2000.

[59] P. Langacker et. al., "Implications of the mikheyev-smirnov-wolfenstein (MSW) mechanism of amplification of neutrino oscillations in matter," Nuc. Phys. B, vol. 282, pp. 589-609, 1987.

[60] B. Aharmim et. al., "Combined analysis of all three phases of solar neutrino data from the Sudbury Neutrino Observatory," Phys. Rev. C, vol. 88, pp. 025501, 2013.

[61] S. Abe et. al., "Precision Measurement of Neutrino Oscillation Parameters with KamLAND," Phys. Rev. Lett., vol. 100, pp. 221803, 2008.

[62] G. L. Fogli, E. Lisi, A. Marrone, D. Montanino, A. Palazzo, et. al., "Global analysis of neutrino masses, mixings and phases: entering the era of leptonic CP violation searches," Phys. Rev. D, vol. 86, pp. 013012, 2012.

[63] K. Hirata et. al., "Experimental study of the atmospheric neutrino flux," Physics Letters B, vol. 205, no. 2-3, pp. 416-420, 1988.

[64] P. Adamson et. al., "Measurement of Neutrino and Antineutrino Oscillations Using Beam and Atmospheric Data in MINOS," vol. 110, pp. 251801, 2013. 
[65] Yoshitaka Itow et. al., "Recent results in atmospheric neutrino oscillations in the light of large $\theta_{13} . "$, Nucl. Phys. Proc. Suppl., vol. 235, pp. 79-86, 2013.

[66] K. Abe et. al., "First muon-neutrino disappearance study with an off-axis beam," Phys. Rev. D., vol. 85, pp. 031103( R), 2012.

[67] M. Apollonio et. al., "Search for neutrino oscillations on a long base-line at the CHOOZ nuclear power station," Eur. Phys. J. C, vol. 27, pp. 331-374, 2003.

[68] F. Boehm et. al., "Final Results from Palo Verde neutrino oscillation experiment," Phys. Rev. D, vol. 64, pp. 112001, 2001.

[69] P. Adamson et. al., "Improved Search for Muon-Neutrino to Electron-Neutrino Oscillations in MINOS," Phys. Rev. Lett., vol. 107, pp. 181802, 2011.

[70] K. Abe et. al., "Indication of Electron Neutrino Appearance from an AcceleratorProduced Off-Axis Muon Neutrino Beam," Phys. Rev. Lett., vol. 107, pp. 041801, 2011.

[71] G. L. Fogli et. al., "Evidence of $\theta_{13}>0$ from global neutrino data analysis," Phys. Rev. D vol. 84, pp. 053007, 2011.

[72] T. Schwetz, M. Tortola, and J. W. F. Valle, "Where we are on $\theta_{13}$ : addendum to "Global neutrino data and recent reactor uxes: status of three-avour oscillation parameters"," arXiv:1108.1376[hep-ph], 2011.

[73] Y. Abe et. al., "Reactor $\bar{\nu}_{e}$ disappearance in the Double Chooz experiment," Phys. Rev. D, vol. 86, pp. 052008, 2012.

[74] F. P. An et. al., "Improved Measurement of Electron Antineutrino Disappearance at Daya Bay.," Chin. Phys. C, vol. 37, pp. 011001, 2013.

[75] J. K. Ahn et. al., "Observation of Reactor Electron Antineutrinos Disappearance in the RENO Experiment," Phys. Rev. Lett., vol. 108, pp. 191802, 2012. 
[76] K. Abe et. al., "Evidence of electron neutrino appearance in a muon neutrino beam," Phys. Rev. D, vol. 88, pp. 032002, 2013.

[77] A. de Gouvea et. al. (Intensity Frontier Neutrino Working Group), "Neutrinos," arXiv:1310.4340 [hep-ex], 2013.

[78] A. A. Aguilar et. al. (LSND Collaboration), "Evidence for neutrino oscillations from the observation of $\bar{\nu}_{e}$ appearance in a $\bar{\nu}_{\mu}$ beam," Phys. Rev. D., vol. 64, pp. 112007, 2001.

[79] R. Tayloe (MiniBooNE Collaboration), "The MiniBooNE experiment: Status and plans," Nucl. Phys. (ProcSuppl.), vol. 118, pp. 157, 2003.

[80] A. A. Aguilar-Arevalo et. al., (MiniBooNE Collaboration), "Unexplained Excess of Electronlike Events from a 1-GeV Neutrino Beam," Phys. Rev. Lett., vol. 102, pp. 101802, 2009.

[81] A. A. Aguilar-Arevalo et. al. (MiniBooNE Collaboration), "Event Excess in the MiniBooNE Search for $\bar{\nu}_{\mu} \rightarrow \bar{\nu}_{e}$ Oscillations," Phys. Rev. Lett., vol. 105, pp. 181801, 2010.

[82] D. Colladay and V. A. Kostelecky, "CPT violation and the Standard Model," Phys. Rev. D, vol. 55, pp. 6760, 1997.

[83] D. Colladay and V. A. Kostelecky, "Lorentz-violating extension of the standard model," Phys. Rev. D, vol. 58, pp. 116002, 1998.

[84] V. A. Kostelecky and S. Samuel, "Spontaneous breaking of Lorentz symmetry in string theory," Phys. Rev. D, vol. 39, pp. 683, 1989.

[85] V. A. Kostelecky and S. Samuel, "Gravitational phenomenology in higher-dimensional theories and strings," Phys. Rev. D, vol. 40, pp. 1886, 1989.

[86] V. A. Kostelecky and R. Potting, "CPT, strings,and meson factories," Phys. Rev. D, vol. 51, pp. 3923, 1995. 
[87] V. A. Kostelecky and R. Potting, "CPT and strings," Nucl. Phys. B, vol. 359, pp. 545, 1991.

[88] V. A. Kostelecky and M. Mewes, "Lorentz and CPT violation in neutrinos," Phys. Rev. D, vol. 69, pp. 016005, 2004.

[89] V. Alan Kostelecky and Neil Russell, "Data Tables for Lorentz and CPT Violation," Rev. Mod. Phys., vol. 83, pp. 11, 2011.

[90] P. Adamson et. al. (MINOS) Phys. Rev. Lett., vol. 101, pp. 151601, 2008.

[91] P. Adamson et. al. (MINOS) Phys. Rev. Lett., vol. 105, pp. 151601, 2010.

[92] P. Adamson et. al. (MINOS) Phys. Rev. D, vol. 85, pp. 031101, 2012.

[93] B. Rebel, S. Mufson, "The search for neutrino-antineutrino mixing resulting from Lorentz invariance violation using neutrino interactions in MINOS," Astroparticle Physics, vol.48, pp. 78-81, 2013.

[94] Sebastian Hollenberg, Octavian Micu, and Heinrich Pas, "Neutrino-antineutrino oscillations as a possible solution for the LSND and MiniBooNE anomalies," Phys. Rev. $D$, vol. 80, pp. 053010 (2009).

[95] L. B. Auerbach et. al. Phys. Rev. D, vol. 72, pp. 076004, 2005.

[96] V. Alan Kostelecky and Matthew Mewes, Phys. Rev. D, vol. 70, pp. 076002, 2004.

[97] P. Adamson et. al. (MINOS), "Measurement of the Neutrino Mass Splitting and Flavor Mixing by MINOS," Phys. Rev. Lett., vol. 106, pp. 181801, 2011.

[98] Joao A. B. Coelho, "Understanding Nu-NuBar Transitions," MINOS Doc-9717.

[99] K. Anderson, B. Bernstein, D. Boehnlein, K. R. Bourkland, S. Childress, et. al., The NuMI Facility Technical Design Report, 1998. 
[100] J. Hylen et. al., NuMI Technical Design Handbook, Internal NuMI report, 2003.

[101] R. M. Zwaska, Accelerator Systems and Instrumentation for the NuMI Neutrino Beam, PhD thesis University of Texas at Austin, 2005.

[102] D. Bhattacharya, Neutrino and antineutrino inclusive charged-current cross section measurement with the MINOS Near Detector, PhD thesis, University of Pittsburgh, 2009 .

[103] L. Loiacono, Measurement of the Muon Neutrino Inclusive Charged Current Cross Section on Iron Using the MINOS Detector, PhD thesis, University of Texas at Austin, 2010.

[104] P. Adamson et. al., "The MINOS calibration detector," Nuclear Instruments and Methods in Physics Research Section A: Accelerators, Spectrometers, Detectors and Associated Equipment, vol. 556, no. 1, pp. 119-133, 2006.

[105] D. Michael et. al., "The Magnetized steel and scintillator calorimeters of the MINOS experiment," Nucl.Instrum.Meth., vol. A596, pp. 190-228, 2008.

[106] G. Drake, J. Dawson, and C. Nelson, "Overview of the MINOS front end electronics for the near detector," 1999.

[107] N. Felt et. al., MINOS Far Detector Electronics User's Manual, Internal MINOS document, 2001.

[108] A.Belias, etal., IEEE Trans. Nucl. Sci. NS-51, 451, 2004.

[109] P. Adamson, J. Alner, B. Anderson, T. Chase, P. Dervan, et. al., "The MINOS light injection calibration system," Nucl.Instrum.Meth., vol. A492, pp. 325-343, 2002.

[110] P. Adamson et. al., "On the linearity of the minos light-injection calibration system," Nuclear Instruments and Methods in Physics Research Section A: Accelerators, Spectrometers, Detectors and Associated Equipment, vol. 521, no. 2-3, pp. 361-366, 2004. 
[111] C. Zeitnitz and T. Gabriel, "The GEANT - CALOR interface and benchmark calculations of ZEUS test calorimeters," Nucl.Instrum.Meth., vol. A349, pp. 106-111, 1994.

[112] P. Adamson et. al. "A study of muon neutrino disappearance using the Fermilab Main Injector neutrino beam," Phys. Rev. D, vol. 77, pp. 072002, 2008.

[113] P. Adamson et. al. (MINOS), "Electron neutrino and antineutrino appearance in the full MINOS data sample," Phys. Rev. Lett., vol. 110, pp. 171801, 2013.

[114] P. Adamson et. al., "Active to sterile neutrino mixing limits from neutral-current interactions in MINOS" Phys. Rev. Lett., vol. 107, pp. 011802, 2011.

[115] P. Ballester, "Hough transform for robust regression and automated detection," Astron.Astrophys., vol. 286, pp. 1011, 1994.

[116] Rudolph Emil Kalman. "A new approach to linear filtering and prediction problems," Transac-tions of the ASME-Journal of Basic Engineering, Series D, vol. 82, pp. 35-45, 1960.

[117] R. Brun et. al. Geant detector description and simulation tool. CERN Program Library Long Writeup W5013, 1994.

[118] K. Nakamura and the Particle Data Group. Review of Particle Physics. Journal of Physics G, vol. 37(7A), pp. 0705021, 2010.

[119] R. M. Sternheimer and R. F. Peierls, "General expression for the density effect for the ionization loss of charged particles," Phys. Rev. B, vol. 3(11), pp. 3681-3692, 1971.

[120] R. M. Sternheimer, M.J. Berger, and S.M. Seltzer, "Density effect for the ionization loss of charged particles in various substances," Atomic Data and Nuclear Data Tables, vol. 30(2), pp. 261-271, 1984.

[121] C. J. Backhouse, Measuring neutrino oscillation parameters using $\nu_{\mu}$ disappearance in MINOS, Ph.D. thesis, 2011. 
[122] M. Campanella, A. Ferrari, P. Sala, and S. Vanini, "First Calorimeter Simulation with the FLUGG Prototype," ATL-SOFT-99-004, 1999.

[123] M. Campanella, A. Ferrari, P. Sala, and S. Vanini, "Reusing Code from FLUKA and GEANT4 Geometry," ATL-SOFT-98-039, 13 October 1998.

[124] G. Battistoni, F. Cerutti, A. Fasso, A. Ferrari, S. Muraro, J. Ranft, S. Roesler, and P. R. Sala, "The FLUKA code: description and benchmarking," AIP Conf. Proc., vol. 896, pp. 31-49, 2007.

[125] S. Agostinelli et. al. (GEANT4 Collaboration), "GEANT4: A Simulation toolkit," Nucl.Instrum.Meth. A, vol. 506, pp. 250-303, 2003.

[126] A. Fasso, A. Ferrari, J. Ranft, and P. Sala, "FLUKA: a multi-particle transport code," CERN-2005-10, INFN/TC 05/11, SLAC-R-773, 2005.

[127] A. Fasso et. al., "The physics models of FLUKA: status and recent developments," Computing in High Energy and Nuclear Physics (CHEP2003) (La Jolla, CA, USA), 2003, arXiv:hep-ph/0306267.

[128] A. I. Himmel, Antineutrino Oscillations in the Atmospheric Sector, PhD thesis, California Institute of Technology, 2011.

[129] H. Gallagher, "The NEUGEN neutrino event generator," Nucl. Phys. B, vol. 112(1-3), pp. 188-194, 2002.

[130] T. Yang et. al., "A hadronization model for few-GeV neutrino interactions," arXiv:hepph/0904.4043, 2009.

[131] T. Sjostrand, S. Mrenna, and P. Skands, "PYTHIA 6.4 physics and manual," Journal of High Energy Physics, vol. 05, pp. 026, 2006.

[132] Z. Koba, H. B. Nielsen, and P. Olesen, "Scaling of multiplicity distributions in highenergy hadron collisions," Nucl. Phys. B, vol. 40, pp. 317-334, 1972. 
[133] R. Merenyi et. al., "Determination of pion intranuclear rescattering rates in $\nu_{\mu}-N e$ versus $\nu_{\mu}-D$ interactions for the atmospheric $\nu$ flux," Phys. Rev. D, vol. 45(3), pp. 743-751, 1992.

[134] C. Alt et. al., "Inclusive production of charged pions in $\mathrm{p}+\mathrm{C}$ collisions at $158 \mathrm{GeV} / \mathrm{c}$ beam momentum," Eur. Phys. J. C, vol. 49, pp. 897917, 2006.

[135] M. Bonesini, A. Marchionni, F. Pietropaolo, and T. Tabarelli de Fatis, "On Particle production for high-energy neutrino beams, Eur.Phys.J., vol. C20, pp. 1327, 2001.

[136] Justin Evans, Measuring Antineutrino Oscillations with the MINOS Experiment, PhD thesis, Jesus College, Oxford, 2008.

[137] Zeynep Isvan, "FHC Systematic Uncertainties,", MINOS Doc-7751, 2011.

[138] P. Rodrigues, "Notes on the Normalization Systematic for NC and CC Analyses," MINOS Doc-6636.

[139] G. J. Feldman and R. D. Cousins, "A Unified approach to the classical statistical analysis of small signals," Phys. Rev., vol. D57, pp. 3873-3889, 1998.

[140] N. Devenish and A. I. Himmel, "Feldman-Cousins for the Antineutrino Analysis," MINOS Doc-5209v7.

[141] P. Adamson et. al., "Measurement of neutrino oscillations with the MINOS detectors in the NuMI beam," Phys. Rev. Lett., vol. 101, pp. 131802, 2008

[142] P. Adamson et. al. (MINOS), "An improved measurement of muon antineutrino disappearance in MINOS," Phys. Rev. Lett., vol. 108, pp. 191801, 2012.

[143] P. Adamson et. al. (MINOS), "Search for the disappearance of muon antineutrinos in the NuMI neutrino beam," Phys. Rev. D., vol. 84, pp. 071103, 2011. 
[144] J. Evans, J. Hartnell and D. Naples, "FHC 7e20 NuMuBar Analysis Position Paper," MINOS Doc-7704.

[145] J. Ratchford, "A k-Nearest Neighbour based Particle Identification Technique for the 2010 Charged Current Analysis," MINOS Doc-7116, 2010.

[146] J. Ratchford, Identifying Muons for Neutrino Oscillation and Cross Section Experiments, PhD thesis, University of Texas at Austin, 2011.

[147] C. Andreapoulos, D. Bhattacharya, et. al., "Updated Cross Section Uncertainties for the Charged Current Analysis," MINOS Doc-2989.

[148] M. Dorman, "Beam Fit Position Paper," MINOS Doc-7146.

[149] Alexander Radovic, "CC binning and decay pipe," MINOS Doc-9672.

[150] Z. Isvan, "Position paper for the non-standard interactions analysis," MINOS Doc8264, 2011.

[151] Joao A. B. Coehlo, "Position paper for a $7.25 \times 10^{20}$ PoT decoherence analysis," MINOS Doc-9118, 2012

[152] W. S. Cleveland, "LOWESS: A Program for Smoothing Scatterplots by Robust Locally Weighted Regression," The American Statistician, vol. 35, pp. 54, 1981.

[153] P. Adamson et. al. (MINOS), "Combined Analysis of $\nu_{\mu}$ Disappearance and $\nu_{\mu} \rightarrow \nu_{e}$ Appearance in MINOS using Accelerator and Atmospheric Neutrinos," Phys. Rev. Lett. vol. 112, pp. 191801, 2014.

[154] Joao A. B. Coelho, "Three and four flavour oscillation results from MINOS," Fermilab Joint Theory and Experimentalist Seminar, 2013.

[155] L.M. Ejzak, "The Search for Neutrinoless Double Beta Decay in CUORE," 2009. 
[156] P. Adamson et. al., "Search for flavor-changing non-standard neutrino interactions by MINOS," Phys. Rev. D, vol. 88, pp. 072011, 2013.

[157] P. A. N. Machado, H. Nunokawa, and R. Zukanovich Funchal, "Testing for Large Extra Dimensions with Neutrino Oscillations," Phys. Rev. D, vol. 84, pp. 013003, 2011.

[158] Jiajie Ling, "MINOS Detector WLS Fiber Attenuation Calibration Documentation," MINOS Doc-4850, 2008.

[159] Sarah Phan-Budd, Luke Corwin, Richa Sharma, Jeff DeJong, Mark Mathis, "Blessed Plots for the Calibration Group," MINOS DocDB 9089, 2012. 